


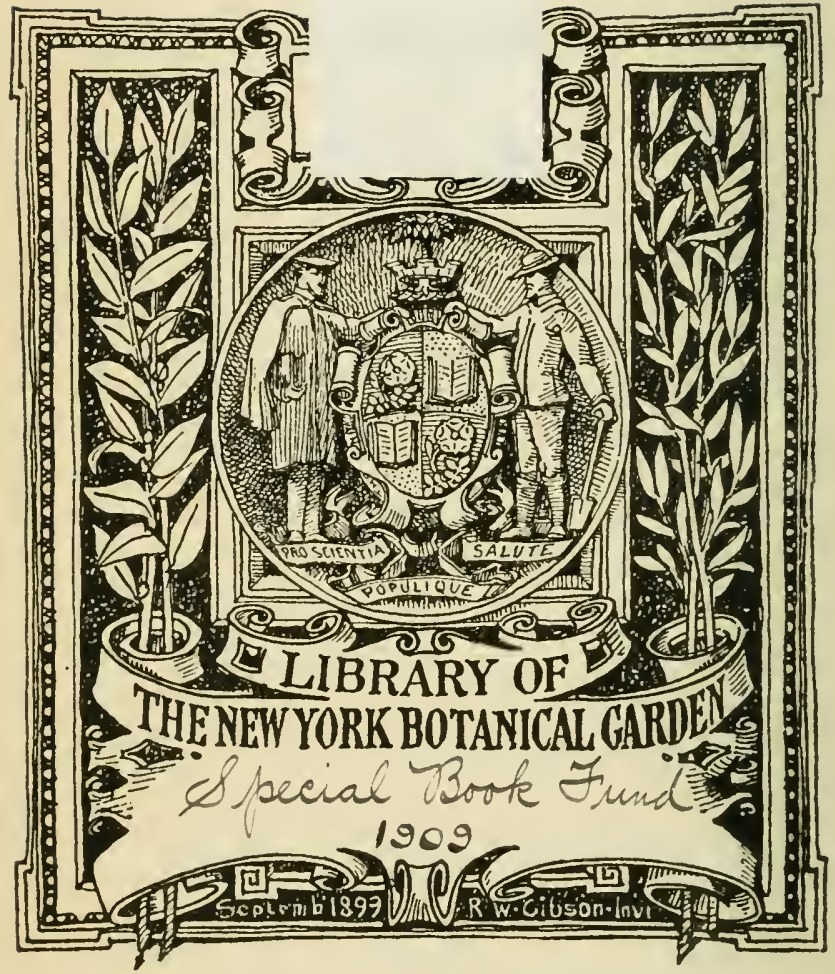




$$
\text { - }
$$









\section{TRAITE DU MAIS}

OU

bLÉ DE TURQUiE. 
DE MNDAME HUZARU (NE VALIAT IA CIAPELIE)

Ruc de l'Eperon, $x^{\circ}$ ? 
- 
PI: 1

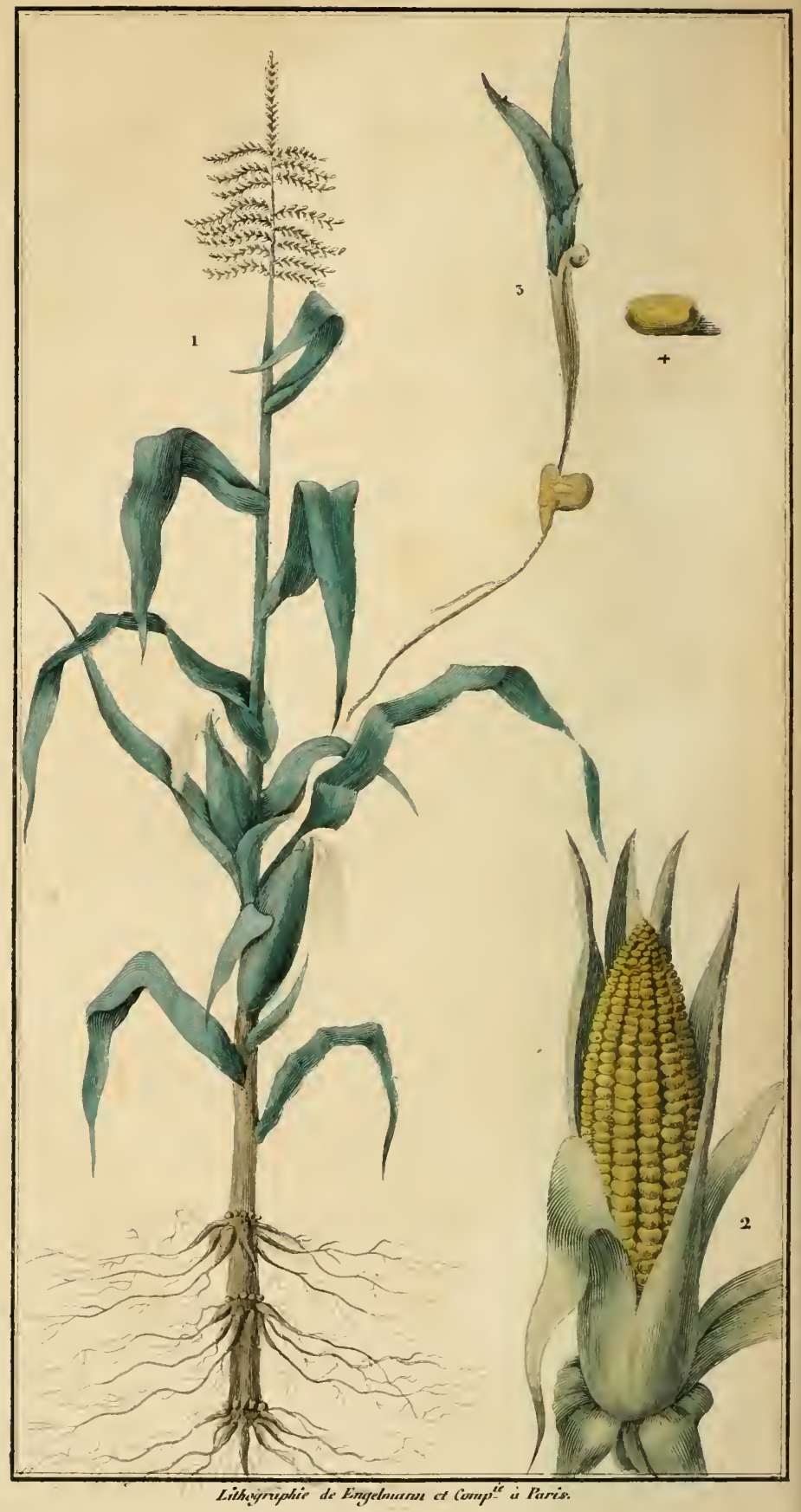




\section{TRAITÉ DU MAIS}

OU

\section{BLÉ DE TURQUIE,}

CONTENANT SUN IISTOIRE, SA CULTURE ET SES EMPLOLS EV ICGXOML WOMESTIQUE ET EN MËECINE.

\section{(O)แrauู}

Auquel l'Aeadémie royale de médecine a dérerné, le: 20 juillet is $\$ 3$, le grand pris fonde par M. Bossaxer pere;

\section{par E.-A. DCchesNe,}

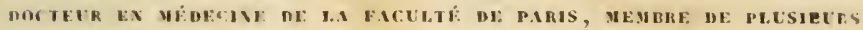
SMCUÉTÉS SAVANTES.

Paus la guerre des États-1 nis, l'Amésieaiu Ilisail au soldat anglais : - Prenez volre froment, el taissct-nous notre mass. . CADFT LIE VALA.

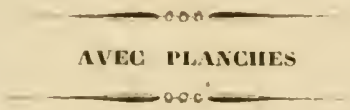

\section{LIBRARY} NEW YORK BOTANICAL GARDEN

\section{PARIS,}

\section{MADAME HUZARI, LIBRAIRE,}

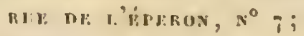

IB I I. I I V KE, I. I I I I E

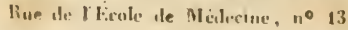




$$
241
$$

08 


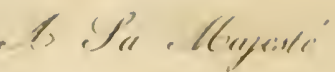

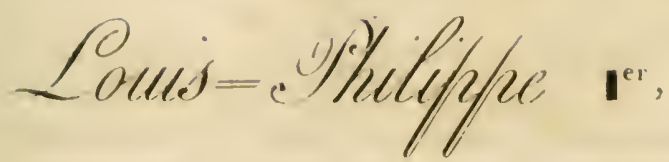

Tove des Francus.

Eire,

En accepteant la Qjédecace de mone

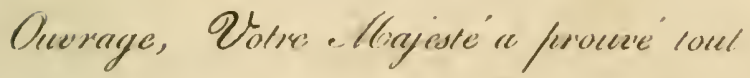

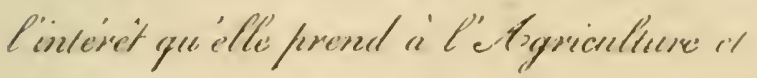

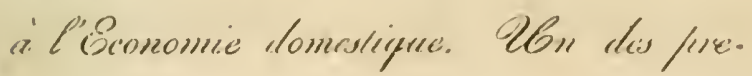


micrs, clle a frut cufleser, avec un grand succai, le elbues dans son berue Parc de . Oeuilly; l'eonneur d'ene furofeager l'ine. sodurion slans quelifues Defiarlemens ori" it ast eniore incomme et d'encouragor sas confelois mullifilies on économie et ene més recine lui élait encore réservé.

QProlecteur des hommes qui se liverent nux Yciencas, elle a droit a' leur cslimee ot a' leur reconnaifsance élernelle.

Soygj assuré, Sirc, de celle Je volre Jevoué Servileur, yecherese. 


\section{AVANT-PROPOS.}

On a lieu de s'étonner de la difticulté qu'éprouve la culture du maìs à s’introduire dans les parties de lá France où il n'est pas encore connu, lorsqu'on a pu apprécier les nombreux usages de cette céréale précieuse, dont toutes les parties sont utiles pour la nourriture de l'homme et des animaux. Cette négligence de la part des cultivateurs pour une plante d'un produit si avantageux est à peine pardonnable, Iorsqu'on les voit, dans certains pays, préférer la 
culture de grains bien inférieurs, le sarrasin, par exemple. Le gouvernement et les corps savans qui s'occupent d'agriculture ont cependant essayé plusieurs fois de fixer leur attention sur cet objet : ainsi, le premier publia, à Paris, en 1786, une Instruction sur les usages du blé de Turquie comme grain.

Cette même notice fut réimprimée et publice de nouveau à Paris par ordre du ministre de l'intérieur, dans le mois de germinal an IV, avec ce titre, Sur la culture et les usages du maïs; elle forme une petite brochure de 32 pages.

L'Académie des sciences de Bordeaux fut, je crois, la première qui s'occupa de cet objet, en 178 ; ; elle proposa un prix sur cette question :

Quel sercit le meilleur procédé pour conserver le plus long-temps possible, ou en grain ou en farine, le maïs ou 
blé de Turquie, plus connu dans la Guienne sous le nom de ble d'Espagne, et quels seraient les différens moyens d'en tirer parti dans les années abondantes, indépendamment des usages connus et ordinaires dans cette. province.

C'est ce concours qui provoqua les nombreux travaux du célèbre Parmentier sur le maïs.

Quclque temps après, l'Académiedes - sciences, belles-lettres et arts de Montauban, voulant aussi apprécier les avantages et les inconvéniens de la culture du maïs, proposa le même sujet au concours.

En 1828 , la Société d'horticulture de Paris publia une Instruction, in- ${ }^{\circ}$ de i p pages, sur la culture du mais ou bléde Turquie et les divers usages de cette plante, avec un programme du concours ouvert pour sa culture. 
La même Société publia, en 1830 , une autre brochure de 3 I pages in- $8^{\circ}$, intitulée, Programme duprix proposé par $M$. Bossange père pour la meilleure culture du maïs dans les départemens de la Seine, de Seine-et-Oise, de Seine-et-Marne et de l'Oise, pendant l'année 183o, et Instruction sur cette culture.

Enfin, le même M. Bossange, dont le zèle ne se ralentit point, fonda, dans l'intervalle des deux concours précédens, un autre prix que l'Académie royale de médecine devait décerner à l'auteur du meilleur Mémoire sur le sujet suivant : Du maïs conme aliment chez lhomme, chez les enfans en bas âge et chez les fenmes qui allaitent. Malgré la position précise de la question, je ne me crus pas obligé de me conformer strictement à sa lettre, ¡'ai fait mes efforts pour atteindre à la 
hatuleur oi étaient parvenus l'illustre Parmentier et le comte Francois de Neulichàteau, c'est à dire que j’ai essayé de meture à la portéedes agriculteurs les différeus procédés ancieusou nouveaux, employés dans des pays fort éloignés les uns des atutes pour la culture du maïs, et d'indiquer les ressources nombreuses que l'on peut en tirer.

J'ai pensé en outre qu'un moyen de rendre mon travail pour ainsi dire plus complet était de ne pas craindre de le voir hérissé de citations, alin que l'on pùt aisément remonter aux sources oi j’ai puisé.

L’Acarémie royale de médecine, daus sa séance du 20 juillet $x 830$, sans toutefois improuver ni approuver le contenu du rapport, qui dommait les plus grands éloges au mais, me décerna le grand prix, qui consistait dans un herbier artiliciel composé des planches 
des Liliacées, peintes par Redouté, d'une valeur de I,400 francs (I), et ordonna en outre l'impression, dans ses Mémoires, de tout ou partie de mon travail (2).

Un deuxième prix d'une moindre valeur a été donné comme encouragement à M. Isidore Gond, propriétaire à Pont-le-Vaux; une mention honorable à M. le docteur Lespès.

Je serai heureux si cet ouvrage sert à introduire la culture de cette plante dans des départemens où elle est peu

(1) Depuis que je m'occupe de la publication de cet ouvrage, M. Bossange a eu la générosité de compléter ce prix en m'envoyant le texte entier, ce qui lui donne une valeur de 2, 400 francs. Je le prie de recevoir ici mes sincères remercîmens pour ce cadeau magnifique et pour les communications bienveillantes qu'il a bien voulu me faire.

(2) La deuxième partie a été imprinée en 1832 dans le premier fascicule du tome deuxième. 
connue, ef si mes concitoyens joignen! aussi leurs suftiages i la décision honorable qu'une des premićres sociétés savantes de la capitale a hien voulu prendre en ma faveur.

Jene puis terminer sans citer, comme. seule récompense des nobles efforts dr. M. Bossange, un passage du discours prononcé par M. le vicomte Héricart de Thury, président de la Société d'horticulture, dans sa séance générale du 15 juin 183 r.

"Une des circonstances remarqua"bles qui caractérisent particulière" ment votre séance de ce jour, c'est "le prix que vous allez déccrner pour " la culture du maïs, prix magnifi" que, vraiment digne de la munifi" cence du gouvernement le plus puis" sant, et cependant fondé par un sim" ple horticulteur, l'un de nos con" frères, respectable père de famille, 
" connu par sa philantropie, M. Bos" sange. 11 m'est bien doux, il est " flatteur pour moi, digne et vénérable " patriarche, d'être ici l'interprète des " sentimens de vos confrères et de vous " prier de vouloir bien en agréer l'ex"pression." 


\section{TRAITÉ DU MAIS}

(1) U

\section{BLE DE TURQUEE.}

\section{HISTORIQUE.}

De: toutes les céréales que l'on cultive habituellement, il n'en est presqu'aucune, si l'on n'en excepte le froment, qui puisse être plus avantageuse que le mais ou blé de Turquie.

Le maïs est la seule gyraminée indigène cultivée en Amérique dans le vaste espace compris depuis le $45^{\circ}$ degré parallile nord jusqu'au 42" parallèle sud. Quoique cette graminéeait étésingulièrement multipliée en Europe, en Asie et en Afrique, l'Amérique a continué de la posséder plus abondamment que toute autre contrée. Cette plante est la base principale de la nourriture des habitans de l'Asie, de l'Afrique et de l'Améripue. Les difl'írens 
peuples de ce dernier pays y sont tellement habitures, qu'ils la préférent à celle que pourrait leur donner le froment.

Voici ce que dit à ce sujet l'illustre Cadet de $\operatorname{Vaux}$ ( 1).

"Le Cincinnatus de l'Amérique méridio"nale, Washington, qui aurait laissé la " réputation du premier des agriculteurs, si " les qualités militaires et les talens politiques " ne présentaient pas l'homme public avec " plus d'éclat à l'opinion, Washington possé" dait vingt-sept charrues; il récoltait par an " 15,000 boisseaux de blé du poids de 60 li" vres, et c'est le maïs dont il faisait sa nour" riture; mais l'Européen trouvait dı pain ") de froment à la table de ce héros-citoyen, " qui n'en mangeait point. Il ajoute : ce " ne serait qu'une opinion isolée; mais j’ai " entend" citer par M. John de Creveceur " ce fait-ci : dans la guerre des États-Unis, " l'Américain disait au soldat anglais : Prenez "notre froment et laissez-nous notre maïs."

(1) Moyen de prévenir le retour des disetles; par Cadet de Vaux. 1812, Paris, in-So, p. 26 et 2.7. 


\section{(17)}

Le célèbre auteurdel'Espritdes lois nt it-t-il pas fait aussi l'eloge indirect du maïs : "Cequi fait, dit-il, qu'il y a tant de nations saurages en Amérique, e'est que la terre y produit elle-nime beancoup de frnits dont on pent se nourrir. Si les femmes y cultirent autour de leur cabane un morceau de terre, le maïs y vient d'abord, la chasse et la péche achèvent d'y mettre les hommes dans l'ahondance (I). ")

En Europe, nos graminées nourrissantes, le froment, l'orge, le seigle courrent de vastes étendues de pays; les terres labourées se touchent nécessairement partout où les penples tirent leur nourriture des céréales. Il n'en est pas de mène dans la zone torride, où l'homme a pu s’approprier des végétaux qui domnent des récoltes plusahondantes et moins tardives. Dans ces climats heureux, l'immense fertilite du sol répond à l'ardeur et à l'humidité de l'atmosphère, une population nombreuse trouve abondamment sa nourriture dans un espace

(1) Montesquieu, Esprit des lois, lir. VII, ch. IX, p. 181, vol. III, édit. Dalilıon, 1827 , in-8". 
étroit, couvert de bananiers, de manioc, d'igname et de maïs.

Telles sont les réflexions suggérées au savant de Humboldt, en faisant la comparaison de notre nourriture avec celle des peuples de la Nouvelle-Andalousie (I).

Chez les peuples sauvages, qui ont à peine quelques ustensiles de ménage, on trouve des vases pour faire leur boisson de mais : ainsi, chez les Indiens Chaymas, leurs hamacs, leurs pots pour contenir le manioc ou le maïs fermenté, leurs arcs, leurs flèches, tout est rangé dans le plus grand ordre.

M. le conseiller Raynhard dit ce qui suit dans son ouvrage, page 325 : "Le blé de Turquie est le principal fruit qui vient sur le terrain sablonneux de notre pays (duché de Bade); nous sommes très bien avec lui (expression allemande) : la bénédiction que Dieu a accordée à notre pays par ce fruit est sans bornes, non seulement le gुrain en est excellent, mais les feuilles vertes et sèches donnent

(r) De Humboldt, Voyage aux régions équinoxiales, t. III, p. I6 et 396 . 


\section{(19)}

une nourriture excellente pour les bestiaus : tout ee que produit ce bli domme a un libonrem. un prolit imonii. "

C'est an Chili que réguaient autrefois, dans le jardin des Incas, les plus beaux maïs du monde. Quand ils y mancpuaient, on en substituait a lemr place qui étaient formis d'or et d'argent, que l'art avait parfaitement imités; ce qui marquait la grandeur et la magnificence de ses souverains. Leurs champs remplis de maïs, dont les tiģes, les fleurs, les épis et les pointes étaient d'oret le rested'argent, le tout artistement soude' ensemble, présentaient autant de merveilles, que les siècles à renir ne verront jamais (I).

Ce passage suffirait seul pour prouver le cas que les pèuples du Nouveau-Monde faisaient du mais; mais on le voit, comme chez nous les métaux précieux, leur servir de monnaie, et même ces peuples instituer des fètes en son honneur (2)

(1) Histoire des Inca.s, traduite de l'espagnol de l'Inca Garcilasso de la Vega, par . B. Baulouin, 1. I, liv. III, ch. XXIV. Amsterdan, Izó, in-ı2.

(2) Id., 1. II, liv. VIII, ch. X 
Chez les Mexicains, on formait avec la pâte de maïs une grande statue qui représentait le dieu Vitzilipultz; on promenaitcette massede farine pétrie en procession, on l'encensait avec de la résine-copal, et on finissait par la découper en morceaux, dont chaque sujet de la domination de Montezuma était obligé d'en manger un, soit dans le temple, soit chez lui, lorsque des infirmités le retenaient dans la maison.

Le maïs faisait la base du pain dont se servaient les Incas pour les sacrifices : ainsi les Péruviens avaient trois sortes de pain. Ils célébraient, au solstice d'été, une grande fête que l'on nommait le Raymi : elle durait neuf jours, pendant lesquels tous les travaux cessaient. Le peuple s'attroupait alors pour faire ses dévotions dans les principaux endroits où l'on adorait les fétiches ou les idoles nationales, et pour se livrer d'abord après à des débauches effrénées, par un scandaleux contraste, dont on retrouve des exemples dans tous les pays de la terre. Le principal acte du Raymi consistait à manger le pain sacré, qu'on appelait cancu ou zancu, dont l'apprêt exigeait beaucoup d'observances vaines et ri- 
dicules; ce pain ne pourail ètre pétri que par les vierges dévouées au culte de Pachacamac ou du Soleil. Ces vierges ne poivaient enire ce pain qu'aprés l'avoir garanti de toute espèce de souillure, et comme lat superstition voit des somillures daus tout, il n'était pas fitcile de rendre la pàte du cancu aussi pure qu'elle devait l'être. Aprés l'avoir partagée en boulettes on en petits gàteaux, on faisait venir. des petits enfans au dessus de ciny ans et au dessous de dix, à qui on froissait le nez et déchiquetait le front avec des pierres aiguisées. Le sang qui découlait de ces blessures était recueilli, et on en arrosait légèrement le pain qu'on distribuait à tous les assistans, qui le mangeaient en présence des idoles, des prêtres et de l'Inea, toujours assidn à présider cette solennité (1).

La deuxième espéce de pain se nommait humintu et se servait dans les festins solennels : leur pain ordinaire se nommait tarutı, il n'y avait pas de différence réelle entre ces

(1) Recherches philosophiques sur les Américains, 1. II, p. 23।. 
trois sortes de pain. Au Pérou, encore dans lí cérémonie des funcérailles, les parens versent, au dessus du liẻu de la sépulture, de ce breurage qu'ils appellent chica, qui, par le moyen de quelques tuyaux, va se rendre dans la bouche du mort ( I).

Les Indiens soumis du Pérou mettent à côté du cadavre des morts, dans les tombeaux, différens comestibles comme des épis de maïs et les alimens qu'ils en préparent. Ils suspendent au pilier qui tient le milieu de la sépulture ( du côté de la tête), et en dehors, plusieurs des choses dont le défunt se servait (2).

Dans le I'oyage de Roulox Baro au Brésil (3), on voit le mil ou maïs servir à un singulier usage parmi les nations Tapuyas. " $J_{t}$ )

(1) Zarate, t. I, p. 56.

(2) Mćmoires philosophiques, historiques, physiques concernant la découverte de l'Amérique, par don Ulloa, t. II, p. 53. Paris, ${ }^{7} 8_{7}$, in- $8^{\circ}$.

(3) Voyage inséré dans les relations vérilables ct curicuses de l'ile de Madagascar et du Brésil, avec l'histoire de la derniere gucrre faite cntre les Portugais st les Hollandais, etc. Paris, $165 \mathrm{r}, 1$ vol. in- $4^{\circ}$. 


\section{$(25)$}

demandais aux 'Tapmies qui avaient amené ces Brésiliens, qui leur arait donné charge d'aller eneillir du mil en mon nom; ils me dirent quils voulaient parachever le: deuil d'un de leur's gens morts, et qu'il fallait du mil afin d'en mèler la firrine avee les os du mort pulvérisés pour les manger. "

"Cependant cenx qui étaient allés à la chasse pendant ce temps du repos rapportèient du mil et des rats, et aussitôt se mirent avec leurs compagnous à piler les os du mort, qu'ils mèlèrent arec la farine de ce mil, ef mangèrent le tout mèli ensemble. "

Le maïs étrit-il connu risant la découverte de ¿Amérique?

Cette question a, jusqu'à présent, divisé tous les anteurs qui ont écrit sur cette cériale. Le docteur Amoreux, entr'antres, dont le mćmoire concourut arec celui de Parmentier, pour le prix proposé en 1784 par l'Académie de Bordeaux, et dont on trouvera un extrait dans le rolume LVIII, mai 1814 , cahier V les Annales de l'Agriculure française, 


\section{(24)}

cherche à prouver qu’il était connu de Pline. M. Desplaces ( I) soutient la mème opinion.

Parmentier, au contraire, dans son excellent, ouvrage (2), combat ses adversaires pied à pied, et prouve qu'il est origginaire de l'Amérique.

- Il n'est plus douteux aujourd'hui parmi les botanistes, dit M. le baron de Humboldt (3), que le maïs ne soit un véritable blé américain.

Lors de la découverte de l'Amérique par les Espagnols, le maïs était déjà cultivé dans la partie la plus méridionale du Chili jusqu'en Pensylvanie. D'après une tradition des peuples atzèques, la culture de cette gुraminée, qui portait dans leur langue le nom de thaolli, en haitien celui de mahiz, et en quichoua celui de cara, avait été introduite du Mexique dans

(1) Histoire de l'agriculture ancienne, extraite de l'Histoire naturclle de Pline, liv. XVIII, p. 49.

(2) Le maïs ou blé de Turquie apprécié sous tous ses rapports, p. 14 et suiv. Paris , 1812 , in-8.

(3) De la Roquette. Histoire de l'Amérique, par Robertson, t. Ier, p. 348 . 
le XVII siecle, par les 'Toulteques, qui ! portèrent en mème temps celle du coton et du piment.

M. Constant-Dunciril ne se prononce pas d'une manière aussi positive; car il dit seulement, dans ses Elémens d'histoire naturelle, que le maïs parait oriģinaire de l'Amériqur méridionale.

M. A. Richard partage la mème opinion.

M. le chevalier de Grégory affirme ( I) que le maïs a été apporté en Italie, dans le XIII" siécle, par les chevalier's des croisades.

Qıant à moi, j’ai fait des recherehes nombreuses et spéciales sur cet ohjet, je n'ai rien urouvédans les auteurs les plus anciens qui ait. pu me faire croire que cette plante ait été connueavant ladécouvertedu Nouveau-Monde.

Joseph d'Acosta (2) assure que le maïs

(1) Annales de l'Agriculture française, III' série, 1. III, P. 76 .

(2) Mistoire naturelle el morale des Indes tant orienlales qu'occidentales, composée en castillan et traduite en frangais par Rohert Regruault Causois, liv. IV, ch. XVI, p 152 et suiv. Paris, 1606 , in-S". 


\section{( 26 )}

tenait le premier rang parmi les substances dont se nourrissaient les Indiens avant qu'ils lussent conquis; qu'ils en préparaient du pain et que ce pain était d'une qualité bien différente de celui qu'on fait avec nos grains d'Europe. Il ajoute : " que le Créateur, en départissant à chaque réģion ce qui lui était nécessaire, avait donné à l'ancien Continent le froment et le maïs au nouveau. "

Le franciscain Bernadino de Sahagun fut un des premiers religieux qui passa à la Nouvelle-Espagne après la conquête terminée, c'est à dire en 1524; il recueillit de la bouche même des Mexicains les plus instruits les détails de la conquête de leur pays par FernandCortès, et lorsqu'il raconte ce que fit Montezuma pour apaiser la colère du dieu Quetzalcoatl (c'est ainsi qu'ils appelaient Cortès qu'ils prirent pour ce dieu voyageur), on reconnait la bonté et les coutumes superstitieuses de ces indigènes.

"Après avoir entendu le récit que lui firent les premiers envoyés, Montezuma rassembla aussitôt quelques devins, des augures et des chefs, ef il les eovoya an port oì étaient les 


\section{$(27)$}

Espagrnols, alin qu'ils s'arrangeassent de mitnière à ce que la nourriture ne manquàt point. anx étrangers, et qu’il eussent tont ce quilis pourraient désirer. Il leur recommanda d'être attentifs à ce qu'ils verraient, et de lui en donner une fidèle relation. Il envoya en outre avec eux quelques esclares, afin qu'on les sacrifiàt devant le Dien qui était arrivé, si l'on voyait. que cela lui convint et qu’il demandàt du sang pour le boire. Ces ambassadeur's s'éloignèrent done et arrivèrent au lieu où étaient les Espagnols, et ils leur offrirent des gyiteaux de maïs (tortillas) teints de sang humain. Quand les etrangers viremt cette nourriture, ils éprouvèrent un grrand dégoùt et commencèrent à cracher et à la rejeter avec horreur, parce que véritablenent le pain sentait le sang. Cela se fit par ordre de Montezuma, et il ordonna de le faire, parce qu’il crnyait que ces étrangers étaient des dieux renus du ciel, et que les nègres étaient des dieux noirs ( 1 ). "

(1) Historia universal de las casas de Nueva Espana. compuesta por el Mi. R. P. Fi. Bernadino de Sahtagmu. C. morceau se trouve dans Anciquilies of Mcxico, b? 
Il est étomnant, comme le remarque très judicieusement M. Robertson (I), qued'A costa, l'un des plus exacts et le plus instruit des affaires de l'Amérique, prétende (2) que le maïs, quoique cultivé sur le Continent, n'était pas connu dans les îles de Cuba, de Saint-Domingue, et où l'on ne mangeait que du pain de cassave.

Martyr, au contraire, qui écrivit après le retour du premier voyage de Colomb, dit ce qui suit (3), à propos de son passage aux Antilles : "ces gens font aussi pain dung formèt qu'ilz appellent maizi, portant lespy long d'une paulme, montāt en aiğu, gros come le bras, ayāt les grrains gros come pois, arrengez lug contre lautre par merveilleux

Augustine Agglio. London, 1830. Il a été traduit en français par M. Ferdinand Denis, et inséré dans la Revue des deux mondes, III année, janvier, p. 74.

( 1) Histoire de l'Amérique, traduite de l'anglais, nouvelle édition, t. II, p. 504 .

(2) Acosta, liv. IV, ch. IV, p. I61.

(3) Extrait du recueil des íles nouvelles trouvées en la grande mer Ocćane, $\mathrm{I}^{\mathrm{er}}$ liv. des Décades, p. 5. Paris, 1532, in- $4^{\circ}$. 
artilice de nature. Icenlx sont blanez deutit que soient menrs, et quitd sont menrs sont fort noirs lesquel\% froissez font farine bläche comme neige "t y a dr ce mai\% asse\% en Granate et à Gennes. "

Voici, an reste, un passage de l'histoire de la vie de Christophe Colomb ( I), qui promre qu'Acosta était dans l'erreur. "Christophe Colomb envoie deux ambassadeurs a un roi présumé dans l’ile de Cuba, lors de la découverte du Nouveau-Monde. Ceux-ci, à leur retom, rapportent qüils ont trouve beancoup de hameaux de quatre ou cinq maisons; à l'entour, étaient des champs plantés d'azi ou poivre doux, de ponmes de terre, de maïs ou blé des Indes, et d'une esprece de lupin. ")

Cettequestion, qui d'abord semble de peu d'importance, présente assez d'intérêt lorsqu'on l'étudie à fond, puisque éest en rassemblant ainsi preuves sur preuves que l'on peut la décider et contribues à faire exactemeut.

(1) Ilistoire de la vie et des voyages de Christophe Colomb, par Washington Irving, 182.8, in-8?. 


\section{(5o)}

l'histoire genérale d'une plante aussi intéressante. Par la suite elle sera beaucoup mieux appréciée par les cultivateurs pour les produits variès et avantageux que ce genre de culture leur fournira, et aussi par les consommateurs, lorsque des essais nombreux et des tentatives coûteuses et souvent sans résultat immédiat leur auront démontré les avantages réels qu'ils peuvent retirer de toutes les parties de cette céréale exotique. Depuis quelques années, les vues de beaucoup de personnes instruites paraissent se porter sur cet objet; espérons qu'elle remplacera bientòt en France certaines plantes qui occupent un grrand espace sur sa surface et qu'elle peut suppléer avec un grand hénéfice.

Maintenant que nous avons prouvé que le maïs était origginaire de l'Amérique, il nous sera facile de suivre son introduction dans beaucoup de parties principales de l'AncienMonde et surtout dans les différens pays de l'Europe, c'est ce que nous ferons dans un des chapitres suivans. 


\section{NOUS IIIGARLS}

l'our faciliter l'itude et les recherches que d'autres uutcurs pourraicne faire par la suite sur le maz̈s Nous donnons ici les noms. vulgaires sons lesquels il est connu dans differens pars.

Allemagne, est et sud. Turkischer weitzen.

Allemagne, ouest et nord. Spanischerou indianischer weitzen.

Angleterre. . . . . Indian or turkey corn. Turckisch. Wheate.

Antilles. . . . . . Maïs ou blé des Inde's.

Arabie. . . . . . Douralı rounny ou elurra rumy.

Archipel indien (grandes tribus de l'). . . . Djagoung.

Autriche. . . . . Kukurutz.

Bohène. . . . . . Turkischer weitzen

Botocudo (Brésil). . . Jitnirun.

Brésil. . . . . . . Ancienuement avali; attuellement millı.

Canada et la Caroline.. . Caracomy.

Chili........ Cua ou қиа.

Chinois des Indes-Orientales....... Famie.

Croatie........ Kuruza

Danemarck. ..... Turkischer weitzen Tytkisk horn.

Egypte. . . . . Hourah châuy on tourky. 
Espagne. . . . . . Mio turquesco.

France. . . . . . . . Maïs, maïz, mahiz, blé de Turquie, blé de Barbarie, blé d'Inde, hlé d'Espagne, blé d'Italie, blé de Rome, blé de Guinée, gros millet des Indes, turquet, turquie (Basse-Bourgogne). Millaral, millargo, millargou (France méridionale )

Géorgie. . . . . . Symiti.

Grèce. . . . . . . . Kalamositaron.

Guarani (Brésil). . . . Abati.

Hollande. . . . . . . Turkisker weitzen.

Hongrie. . . . . . . Kukuricza ou tengeri.

Italie. . . . . . . Grano di Turquia, formentone.

Indes-Orientales. Malais. Jagon.

Japon. . . . . . . S Sjokusa ou too kibbi.

Lombardie. . . . . . . . Sorgo turco, melgone.

Mexique (anciens habitans). . . . . . Tlaolli.

Mingrélie. . . . . . Lazouti.

Mississipi (les sauvages du ). . . . . . Ewahim neasch.

New-Yorck (anciens habitans). . . . . . J Jaeskuıg. 
Omagua (Brésil ). . . Mhuti.

Otaïti. . . . . '. 'ourina.

Pays-Bas. . . . . . Turckskoren.

Pérou. . . . . . Z . Zara ou Cara.

Perse. . . . . . Hildèh.

Pologne. . . . . Przenica turecka.

Portugal. . . . . Milho de Turquio.

Possessions portugaises en

Afrique et en Asie.. Miģlio zaburn.

Russic........ Kukuruza, tourtoia, abscsunka.

Styric inférieure. . . . Kukurut\%.

Suede. . . . . . . Tyrkisk korn, turkischer weitzen.

Suisse et bords du Rhin. Valech korn.

Toscane. . . . . Grano siciliano.

Turquie. . . . . Kukuru, rous.

Virghinic. ...... Pagatowr.

Latin. . . . . . Frumentum indicum (1), Frumentum turcicum (2), Turcicum frumentum (3), Triticum indicum (4), Zea maï (5).

(1) Matliole, Camerarius, Tabernamontanus , G. Bauhin, Mćrian, Morison.

(2) Lonicer, Dodoeus, Gérard.

(3) Fuchs, Tragus.

(4) Dalechaıp, J. Bauhin.

(5) Linné, Thunberg et les botanistes moslernes. 


\section{$(34)$}

L'origine de la dénomination de blé de Turquie, quien France on donne le plus généralement à cette plante, n'est pas encore bien connue; peut-être que ce que je vais en dire paraitra plus probable que l'explication que quelques anteurs ont vouh en donner jusquà ce jour.

Les Espagnols possédèrent de tout temps les royaumes de Naples et de Sicile, nul doute qu'ils n'aient cultivé le maïs bientôt après la découverte de l'Amérique, parce que les Toscans, qui ont reçu le maïs des Siciliens, le nomment encore grano siciliano. Les Véni tiens, les plus grands marchands de ce temps, qui possédaient en Grèce une grande prartie du Continent etbeaucoup d'iles, et qui faisaient un commerce considérable arec les ports d'Italie et la côte gुrecque située vis à vis, transportèrent aussitôt cette plante en Morée, à Chypre, Candie et dans les iles de li mer Ionienne, d'où clle fut bientòt répandue par les Grees dans tous les pays habités par eux ou par ceux qui suivaient le rhythme grec, tels que la Bosnie, la Servie, la Croatie, la Sclavonic et la Hongrie. Il a pu en résulter que, dans ces pays soumis 


\section{$(55)$}

•anx Tomes, lo malis ait equ plantio dans les temps les phus recules, et que les autres mitions, telles you les Mllemands, les Fancais et une partie des habitans de l'Italie, ne surent donner d'antro nom que eolui de blé de T'urquie à me plante quils avaiont monve che\% les 'Tures, on yui lem ariat chi apportíe par des marehands fures sams designation de nom.

Camerarins, midecin dr Nuremberg, l. nommait dejà ainsi en 1586 (1). Il parait que la culture du maïs a précéde de beancomp, en Espagne, celle des pommes de terre.

En tous cas, l'utiliti bien recomme de cette plante par le peuple américain a dû contrihuer beancoup à en rendre sa propagation très prompte chez les différens peuples dont nous vemons de parler.

\section{Descriptinn botumique.}

Le maïs ou blide Turpuic, zea may's, L. (2),

(1) Deplantis Epitome utilis Mathioli et locupletata (amerario, p. 186, anmće 1586.

(2) Linné, Spcc. I 133. Lamarck, Dicl. 3, p. 680. 
a déjà été sourent ot anciennement représenté par des figures. La meilleure est celle de Morison ( $\mathrm{r}$ ): c'est une gravure sur bois. Blakw (2), Fuchs, Mathiole, Camerarius, Dalechamp, G. Bauhin, J. Bauhin, Mérian, et beaucoup d'autres, en ont aussi donné des figures; mais presque toutes ont été copiées servilement, celle que l'on voit dans Tournefort est déjà beaucoup mieux (3). On en trouve une qui laisse de bien loin en arrière ce qui avait été fait jusqu'à présent; elle est jointe à l'ouvrage de Boitard sur les Prairies artificielles : celle qui est représentée à la lin de cet ouvrage lui est encore bien supérieure. (Voyez $\mathrm{Pl}$. I.)

Cette plante fait partie de la Monoëcic triandrie, I. (fleur's unisexuées), de la quin-

De Candolle, Flore francaise, t. III, p. 98. Gæru1., Fruct. 1, t. I, p6 6. Fuchs, Hist. 473 , rc.

(1) Hist.plant. ed lat., fol. 1715 , t. III, p. 248, sect. 8, tab. 13, fig. I, 2, 3 .

(2) Herbarii Blackwclliani auctarium, collectio stirpium, etc. Novimbergæ, $177^{3}$.

(3) Joseph Pitton de Tournefort, Institutiones rei herbaria, 1700, in-4" , p. 531, et tab. 3o3, 3\%4, 305. 


\section{$(57)$}

zième classe de 'Tournefort (plantes apétales), des graminćes de Jussicu (plantes monocotylédones, monohypogyurs), et de la huitième classe de A. Richard.

Description du gros maïs.

Plaute annuelle ( $\mathrm{r}$ ).

Chaume haut de 6 ì 8 pieds, raneux, glabre, cylindrique. (Cete tige, raide et solide, est remplie d'une moelle fongueuse, blanche, sueculente, d'une saveur donce et sucrée quand elle est verte.) Feuilles alternes, engainantes, longues de 2 pieds et plus, largres d'environ 2 ou 5 pouces. Fleurs monoïques dans des épis séparís, fleurs màles, qui sont d'un blane verdàtre, ou quelquefois légèrement purpurines, oceupant la partic supérieure de la plante, où elles constituent une très grande panicule ramense, formée par la rémion d'un grand nombre d'épis recourbés à leur partie supérieure. L’axe de ces epis est long de 8 à 10 pouces, flexuenx, pu-

(1) Richard, Mistoire nalurelle módicale, t. I, p. 344 
bescent. On trouve à chaque dent de l'axe deux épillets biflores, dont l'un est sessile et l'autre pédonculé.

L'épicène bivalve, billore, valves un peu inégales, l'extérieure plus grande, plus pubescente que l'intérieure, toutes deux carénées. Glume également bivalve. Paillettes très minces, membraneuses et transparentes. Les étamines, au nombre de trois, sont pendantes.

Fleurs femelles situces au dessous des mâles, aux aisselles des feuilles. Elles sont réunies par des liggnes longitudinales sur une sorte d'axe charnu, conoïde. Cet assemblage ou eapitule allongé de lleurs est enveloppé d'un grand nombre de feuilles vaginantes, qui se développent très tard.

Chaque épillet est biflore, mais l'une des deux fleurs avorte constamment et est rudimentaire; l'épicène est à deux valves arrondies, obtuses, plus courtes que l'ovaire; la glume offre deux paillettes plus petites que l'épicène.

Ovaire ovoïde, glabre; style court, glabre; stigmate filiforme, plumeux, très mou, long de 8 à 10 ponces; la réunion de ces stig- 


\section{( 59$)$}

mates forme une harbe cpaisse, molle et charune, qui prend par dessus la partie supérienre de la gaine foliacée qui entoure l'assemblage des fleurs. Le finit est irreggulièrement arrondi, comprimi vers sa base, e'est un des plus gros de toule la famille. Les racines sont nomhreuses, dures, fibrouses, blanches et mennes; elles s'étendent plutòt quidles ne pivotent.

Les épis ont ordinairement douze rangées de trente-six graius; ce qui, à denx épis par pied, aussi terme noyen, dome un produit de huit cent soixante-quatre pour un. Les poIygones de douze à quatorze côtés sont cenx (qui dominent dans les épis de blé de Turyuie ( 1 ).

\section{Vurićtés.}

On distingue aujourd'hui en France nois varictés bien distinctes de maïs, savoir :

1 . Le grand maïs; 2 ". le maïs quatan-

(1) Ch. Bonnel, Recherclics sur l'usage des fouilles dans les planies, p. 3oli Gotlingue et Leyde, 1754 . in- $4^{\circ}$. 


\section{(40)}

tain; $3^{\circ}$. le maïs à poulet. Nous décrirons l'une après l'autre ces trois variétés.

$I^{\circ}$. Grand maïs; fuzaro (italien).

C'est le plus tardif, mais il est aussi le plus productif. On dit que, dans les terres vierges des.États de l'Ouest de l'A mérique septentrionale, il s'élève jusqu'à 18 pieds, et on en a observé de la moitié de cette hauteur dans les. fertiles vallées volcaniques du Vicentin (I).

Dans le Pérou, en Guinée, chaque tige, qui atteint quelquefois $\mathrm{s} 8$ pieds de hauteur, porte dix à douze épis, et chaque épi environ mille grains (Amoreux).

Ce maïs est celui dont il existe maintenant de grandes cultures en France; il est plus vigoureux et plus fécond, puisqu'il demeure davantage sur terre; on le voit surtout dans les pays un peu méridionaux, où il peut arriver à maturité.

Il en existe de plusieurs couleurs :

${ }^{\circ}{ }^{\circ}$. Du jaune.

L'écorce de sou gyrain est épaisse ; sa farine,

(1) Nour'au Cours somplet d'agriculture, t. IX, p. 2.75 . 


\section{(41)}

plus pàle, n'a pas autant d'odeur que celle du maïs quarantain jaune.

2". Dublanc.

Les Américains de la Nouvelle-Yorck, dit Parmentier, P. 4\%, préférent le maïs blane à tous les autres; et lorsqu'ils n'ont récolté que du maïs jaune, ils le vendent pour en acheter du blanc, dont la galette, selon eux, a une meilleure qualité.

S’il s'agissait de prononcer si cette préférence est fondée ou non, nous serions embarrassés, puisque l'analyse ne présente aucune différence essentielle dans la nature et les proportions de leurs parties constituantes.

$3^{\circ}$. Des épis mélangés de grains rouges et de grains blancs.

$$
\begin{aligned}
& 4^{\circ} \text {. Du rouge. } \\
& 5 \text {. Du bleu. } \\
& 6^{\circ} \text {. Du violet. } \\
& 7^{\circ} \text {. Du noir. } \\
& 8^{\circ} \text {. Du panaché. } \\
& 9^{\circ} \text {. Du manbré. }
\end{aligned}
$$

Ces conlenrs se rencontrent dans le mème. champ, et quelquefois sur le mème epi.

On cultive, dans nue partie du Limguedore or 


\section{(42)}

principalement aux environs d'Albi, un mais dont l'épi n’a que huit rangées; le grain est plus gros et l'épi dépotillé plus petit, on l'appelle mä̈s de Padies. Dans d'autres endroits, on préfère le maïs à seize rangées, qu'on distingue sous le nom de maïs de Cussac. L'épi dépouillé est plus gros et les grains plus petits, mais la pesanteur spécifique est à peu près la même d'après Saint-Genis, ce ne sont que de simples variétés.

Le maïs blanc est de quinze jours plus hàtif que le jaune en France. On le préfère dans le département des Landes, des Hautes et BassesPyrénées, où il passe encore pour être le plus ¡roductif; il réussit dans les terres fortes. Le jaune, au contraire, exige un sol moins gras, et sa culture est plus généralement adoptéedans les départemens de la Gironde, du Rhône, de Saône-et-Loire, de la Côte-d'Or et du Doubs( ( ) .

$2^{\circ}$. Maïs quarantain, quarcentino, cinquanlino italien ).

On l'appelle aussi petit maïs, maïs précoce;

(1) Article Maïs, Diclionnairc d'Ilistoire nalurclle, t. XIII, 1803 , publié par Déterville. 
mona con Amérique, e'est à dire matis de denx mois; Turpuie de Romanie on d'Espagnor (Bourgogne).

Son frain est beanconp plus petit, mais il est plus hàlif; son écorce est très fine; sa fatrine est d'un bean jaune, et d'mne excellente ockenr. Sa rige ne s'eleve pas plus haut que 4 pieds.

Les expéricuees de C!nancey, Sageret et Parmentier prourent que sa maturité ne devance guère celle du maïs ordinaire que d'un mois au plus dans le climat de Paris et de deux au midi, mais (que sa regétation est beanconp) moins vigoureuse; ses grains sont plus petits, en le plantant plus serré ses produits se rapprocheraient.

50. Le maîs à poulet.

Il est encore plus petit et plus precoee que le quarantain. Son épi n’a environ que 5 pouces de long et n'oflie que huit à dix ranges de grain. Il y en a du jaune et du blane; il pent arriver à entiere maturité en deux mois sous le climat de Paris, à compter de l'ípoque des semailles. De quefle milite ne deviemdrait pas le maïs prócoce, sil étail atussi commmun quil 


\section{(44)}

parait l'être dans les différentes contrées de l'Amérique? Sa culture pourrait s'étendre partout où le climat trop froid ne permet pas au maïs tardif d'être planté dans les premiers jours de mai et de mûrir avant les gelées d'automne. Il serait même possible de le faire succéder à certaines productions hâtives dans le midi de la France.

Nous allons parler actuellement de quelques autres variétés que les auteurs indiquent, mais qui ne sont pas encore assez connues pour qu'il soit possible de les classer bien distinctement.

Une espéce de maïs a reçu le nom de pierre ì fusil, à cause de la dureté de son grain. Les pays les plus fertiles en maïs, conme la Virginie, par exemple, en produisent une variété dont le gyrain est aplati et très grand, dont la tige et les épis acquièrent une longueur double de celui d'Egypte.

En ı 818, on a rapporté de la Pensylvanie une nouvelle espèce de maïs, qui ne s'élève pas autant que celui recueilli à l'embouchure de la rivière d'Hudson; il ne domne qu'un ou deux epis, comme notre mails commun; il a 


\section{$(45)$}

moins de fenilles, surtoun il l'endroir de l'épí, mais il pousse par le pied jusqu à lunit ou dix antres tiges secondaires, portant chacune un ajpin peu moindre que celui de la tige principale : ce maïs muirit facilement et produit beaucoup; il a parfaitement réussi ì Grenade, département des Landes, che\%. M. SaintAndré, et a Lilpuyades, departement de la Haute-Garonne, che\% M. Cazals (1).

Fen M. Andrieu de Cheptainvillea montré, dans une séance de la Socicti d'agriculture de Seine-et-Oise, un echantillon d'une antre espèce de maîs nomme petit maïs mandan on molamais, qu'il a cultive pour la première fois en rsig, par forme d'essiti. Cette nomvelle esprece, qui lui a été domuée par M. Michant, prosenait de Baltimore, ot elle at éti apporte du pays des siluriges ntandimmes, qui habitent sur les bords du Missouri, dans lit IIauteLouisiane, pays très froid et situe i près de 1,000 lieues de la mer. Ses grains étaient. hancs comme ceux qu'il arait recus, à l'ex-

(1) Bubliothique phisiro-iconomique, t r. p. 250. 1819 , in-12. 


\section{$(46)$}

ception de deux ou trois épis dont quelques grrains étaient couleur d'ardoise et le reste de couleur jaune. La maturité a été plus tardive d'environ vingt jours; peut-être que plus, long-temps acclimatée, cette variété produirait une conleur fixe et mùrirait plus tôt.

Quant à l'espèce dont l'épi est rameux, qui parait exister en Amérique et dont on a dẹjà vu plusieurs pieds en France, entr'autres chez M. le comte Francois de Neufchâteau, il faut attendre qu'elle soit mieux connue (I).

Molina décrit d'une manière très courte. une variété à feuilles dentelées, qu'il a trouvée au Chili; mais aucun autre auteur n'en a parlé depuis lui (2).

J'ai eu en ma possession, en 1829, un épi de maïs que l'on m'avait envoyé des environs d'Auxonne (Côte-d'Or'). Sa forme était exactement sphérique, de la grrosseur d'un fort abricot. Je regrette ne plus l'avoir eu à l'époque

(I) Biblinthèque physico-économique, 1818 , juin, 1). 401 .

(2) Histoire naturelle du Chili. 


\section{( 17 )}

des semailles de 1830 ; je l'avais emroyéa l' $\Lambda$ catdémie rovale de médecine, il ne m’a pas étí resdu. La récolte m’aurail démontré si c'étail une variéti on un simple jeu de la nature.

M. Bonafous cite mne variétc qu'il nommor maïs de Californic el qui lui a offer les diffirences suivantes (1):

$1^{\circ}$. Les gaines qui enveloppent le chaume, les spathes qui recourrent l'épi sont heirissées de poils raides et nombreux, de la longuem de 2 à 5 lignes, et les glumes de la panicule extrèmement vehues.

$2^{\circ}$. Les feuilles, garnies de poils à leur surface supérieure, sont toutes pendantes parallìlement au chanme, depuis leur premier développement, et leur inclinaison est telle, yur les languettes existantes à leur aisselle so trouvent eonstanment dieouvertes.

$3^{\circ}$. Un caractire non moins important eonsiste dans l'insertion immédiate des épillets màles sur le rachis ou axe de la panieule. Tous

(1) Annales administratives le l'agriculuure francaiss. IIIe série, I. IV , v. I 45. 


\section{$(48)$}

les épillets, à l'exception des plus inférieurs, sont sessiles et très rapprochés, au lieu d'être pédicellés et écartés comme dans l'espèce ordinaire, où l'un des deux on trois épillets de chaque groupe est toujours porté sur un pédicelle.

$4^{\circ}$. De plus, il a observé qu'un grand nombre de fleurs ne renferment que deux étamines au lieu de trois, que l'on trouve constamment dans l'espèce commune.

5. La couleur des anthères est d'un janne doré, au lien d'être légèrement purpurine ou verdàtre.

$6^{\circ}$. La graine, d'un blane nacré, jaunâtre, est un peu allongée et translucide.

Cette variété s'est reproduite pendant plusieurs années, sans changement, dans le jardin botanique de Lyon.

Kalm dit qu'on ne trouve pas de maïs sautvage en Amérique; cependant Hernandez (1) en cite une espèce.

On cultive en Piémont, depuis des siècles,

(1) Rerum mod. Hisp. Thes. Rom. I65! , liv. VII, p. 2.42 . 
le maïs quarantain; ependant il n'a jamais cru spontamement dims ancmu endroit (1). Il ne fant jas confondre, avee le nails dit aussi ble de Guince, le matis de Guince, mö̈s Guinee (hot.), nom espagnol de la flore du Péron et qui a à peu près les mèmes usages. De ses grains rotis, nommés dans le pays crincha, on retire une farine tres hlanche dont on fait la boisson dite ullpu et l'aliment mUะเทักเ (2).

\section{Analyse.}

L'analyse du mais a dejà été faite par plusieurs auteurs. Burger, deVienne(5), s'en est occupé d'me manière assez suivie; je donnerai, ici, le resultat de ses travaux.

(1) Instruction dic professcur Buniva, p. 315.

(2) Diclionnaire des Sciences nalurelles, 1. XXVIII, p. 113.

(3) I raité complet sur l'histoire nalurelle, la cullure, l'cmploi du maïs, par Jean Burger, p. 3ı j-3 fo. Vieune, i 809 , in- $S^{\circ}$ (en allem.). 


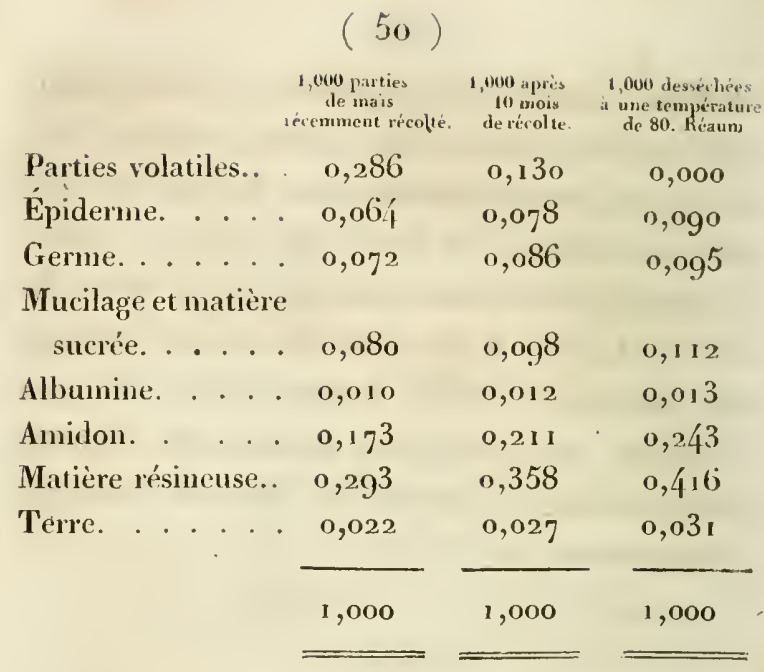

Deux pages plus loin, il assure que, d'après ses expériences, il ne doute plus que le maïs ne contienne du gluten, il l'a peut-être confondu ici avec la matière résineuse. Le boisseau de Vienne de 5,537 pouces cubes, anciens pouces cubes français, qui, d'après le calcul de Vega, contient 6r 499, 49 cubes, pèse, lorsqu'il est rempli de vieux maïs de sept mois, 85 à 86 livres; avec le maïs d'une année, 87 à 88 livres; avec du maïs séché à 80 degrés Réaumur, $9^{6}$ à 97 livres.

M. le docteur Lespez a fait, aidé de 
M. Mareadieu, éleve de M. Vauquelin (1), l'analyse de la farine de mais. Voici le risultat qu'ils ont obtem :

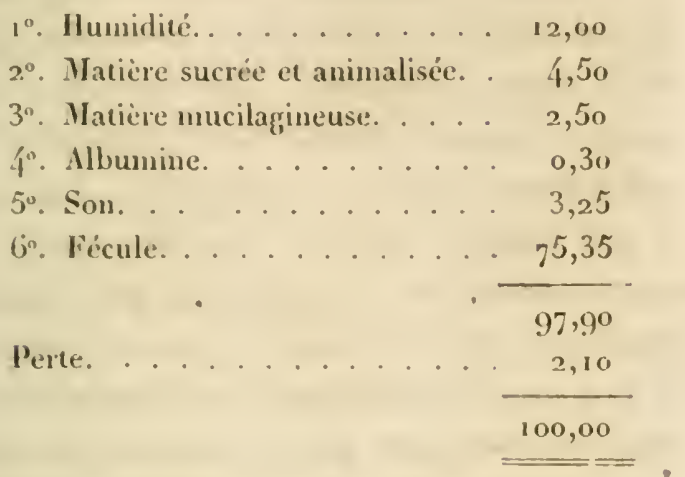

Le résidu saliu obtenu par la calcination de 5 grammes de farine pesait ro centigrammes. Il se composait de sulfate de potasse, d'hỵdrochlorate de potasse, d'hydrochlorate de chaux, de carbonate et de phosphate de chaux. Le dernier y dominait. D'aprés cette analyse, on voit que le maïs ne contient pas de gluten et qu'il diffère en cela du fromeut qui

(1) Thèse, 1825, p. 17. Vovez la T'able. 
en contient 1 2,5 sur 1000. Cependant, si l'on consulte l'ouvrage de Marabelli ( $\mathrm{I}$ ), on y voit le corollaire suivant. "Cor. VII, p. Lxvi. "Zece mays seminum farina ab illa tritici discriminatur, quòd prima minorem exhibeat amyli portionem, dìm majorem suppeditat copiam saccharince substantice, et quòd gluten zere mays substantice resinosre, à quâ colorem accipit, commisceatur, neque prceditus sit eâ elnsticitate quam gluten. triticum, quòd albidius est, prce se fert."

Les produits indiqués par Marabelli sont assez curieux pour que je continue à le citer. " Ulterius processii quamdam ejusdem succi portionem ad julapii consistentium evaporationis ope perduxi, sibique commisi, ut si fortè fortuna saccharina qucedam portio detegenda sese crystallizatione offerret, cognoscerem; et reapse post áliquot hebdomadas obtulit nonnullam crystallince forme materiem, vel potius crystalli grumos, qui à liquore consistente secreti ne

(1) F. Marabelli. De zeá may's plantấ analytica Disquisitio. Pavie, $179^{3}$, in-8 . 


\section{$(53)$}

hilum quidem existimari poterant diversi ab aliqui communis ustis specie, etc. " (P. xıx.)

Corollaire I, p. Lxm.

"Zece mays analysis saccharinam preebuit substantiam paritate pliss minus diversam, ex quä acidum sacchari, etc. "

Corollaire II, p. Lxill.

"Singule quas examinavi hujus plante partes (culmos, semina lacte adhiuc ürgida, medullam receptaculi), quanquam different relativa horum principiorum proportio, omnes tamen continebant it saccharum et exposita salia, easdemque terras. "

\section{Corollaire VIII, p. Lxvil.}

"T'andern alique zee mays plantee parıes, quanquam relate ad culmum qualitate nonnihil puriorem suppeditare valeant saccharinam substantiam, vincuntur tamen ab ipsarum culmis qui majorem pre cateris copiam suppeditunt et talem ut ne ulla, si veros saccharinos culmos excipias, alia ve- 


\section{$(54)$}

setabilia, que sacchurum valent producere, ipsis sint pro sacchari contenta nec qualitrte nec quantitate comparanda. Culmorum etenim sece gratum dulcissimum succum in jucundum syrupum facili negotio convertimus, nec saccharino inferiore nota obtinemus, ad reconomicos usus optimum et sacchari, ubi inspissemus, vices gerentem. Quamobrem mihi non leve obortum est dubium, posse haec ex culmorum succo saccharina producta su'stantia saccharo vero venali apud nos substitui gravibus sumptibus parcendo, ipsius precipuè uunc inspecto pretio. "

Buniva, d'après Marabelli, admet aussi tans le maïs la présence d'une substance glutineuse.

M. Raspail, naturaliste distingué, pense aussi, d'après ses expériences, que le maïs contient du gluten.

Le mais, dit A. Richard ( $r$ ), ne contient pas de gluten; mais M. Bezio a trouvé dans cette farine une matière particulière, qu'il a nommée zéine, qui paraît avoir quelqu'analogic'

(1) Elém.d'hist nat.mür, art.Mrïs, t. 1, p.344.1831. 


\section{$(55)$}

avec le gluten : elle est jaune, molle, malliahle et élastique, insoluble dans l'ean fioide, se ramollissant dans l'eau chande, soluble dans l'alcoul re les huiles.

Le doctem John Graham, professem de l'Universite d'Harvard anx Etats-Unis, a fir lanalyse chimique du maïs, et il y a reconnu un principe grossier analogue à cehui quw V. Proust avait trouvé dans l'orge; la scine est dans le maïs ce qu'est l'hordéine dans l'orge, et s'obtient par de semblables procédés, mais elle y est peu abondante. En voici le résultat :

$$
\text { Maus a l'état frais. Muls ser }
$$

Eau

9,00

Fécule amylacié.

77,00

$84,5 y 9$

Zéine (matière particulière).

Albumine.

3,00

3,296

2,50

2,745

Matiere gommense.

$1,-5$

1,922

Sucre.

1,45

$1,59^{3}$

Principe extractif.

0,8 o

o,

Enveloppes et mat. ligneuse

3,00

3,296

Phospliate, carbonate, sulfate de chanx et perte. . .

$$
\frac{1,50}{100,00}-\frac{1,648}{199,98011}
$$

(1) Junural de I'burmate', 1. 111, 1828, 1. 3i1 
Il faut, je crois, conclure de ces opinions différentes que des expériences nouvelles sont nécessaires; mais il serait utile d'indiquer alors avec soin quelle espèce de maïs on a soumise à l'analyse, depuis combien de temps le grain était récolté, et quelle était la nature du sol qui l'a produit.

\section{Des accidens du maïs.}

Le maïs étant une plante inconnue pour beaucoup de cultivateurs, il faut leur indiquer avec soin les différens accidens auxquels il est exposé.

Il craint la trop grande humidité; aussi fant-il l'éloigner des endroits bas, qui sont souvent submergés par des débordemens. C'est dans ces lieux surtout qu'il pousse davantage; mais alors ce n'est, pour ainsi dire, qu'aux dépens de la matière sucrée et de la bonté des épis qu'il doit produire, il est étiolé. Les longues pluies, les inondations lui sont préjudiciables, surtout dans les terrains forts et argileux; le cultivateur doit prendre toutes ses précautions pour faire ecouler ces eaux ic plus tôt possible. 


\section{( 57 )}

II ne redonte pas moins l'extreme sécheresse : "C'est alor's surtont, dit l'illustre "Parmentier, quil fant prendre farde de " trop travailler la tere, parce que, le pied et " la racine se desscichant, la plante soulfri" rait (1). "Si la chaleur du jour se prolonge pendant asse\% long-temps, et que les nuits me viennent pas rafraichir la plante, on la voit bientot langurr, les fenilles se foncer et jaunir: un mode d'irrigation bien entendu pent ètre urés utile dans eette occasion.

Les départencus de l'Ariége, des Hantes et Basses-Pyrénces doivent l'ahondance de leurs recoltes encore plus à l'avantage de l'irrigation qu'à la smpériorité du sol; ils ont l'ean à lemr disposition dans des reservoirs qu'ils remplissent de toute celle que lent ancnent les nombreux torrens de leurs montagnes, et lorsque la scicheresse commence à attrister les plantes, ils inondent lems champs.

Un froid trop vif et prolonge mit an mais do plusiems manieres: "i la fin te l'hiver, "n

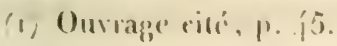


empèchant de semer; $2^{\circ}$ au printemps, lorsqu' il est sorti de terre et qu'il végète, en retardant sa marche; $5^{\circ}$ à l'automne, en l'empèchant de parvenir à maturité : aussi, pour prévenir deux de ces inconvéniens, que l'on peut craindre davantage, parce qu'ils se reproduisent plus sonvent dans les pays placés comme ceux des environs de Paris, il faut choisir les espèces hâtives qui parviennent à maturité en quarante ou cinquante jours. Mais si le froid et les pluies continuelles ou la bruine surviennent après la moitié du mois d'aoùt, et si la mauvaise saison continue à endommager le maïs qui a été planté trop tard, il conviendra alors de le couper, quoiqu'il ne soit pas mûr; car, dans ce cas, les feuilles s'appauvrissent et deviennent blanchàtres, et les grains ne mùrissant pas, on ne peut les conserver. Cependant si, lorsqu'on coupe les grains, ils étaient un peu mûrs, on doit alors les employer les premiers à manger ou à nourrir la volaille.

Le vent, surtout lorsqu'il est accompagni d'orage, cause souvent de grands ravages dans les champs de maïs, accident auquel on peut remedior quelquefois en ur semant 
pas le grain trop écarté, en ayant soin de bien buter les pieds et de ne pas semer sur des endroits trop élevés et par conséquent trop exposés à son action. Plus la tigge du milis rst d́devée et plus elle est sujette aux ravages des grands vents. II ne faut qu'un instant pour concher tout un champ sur terre. Dans une occasion pareille, un cultivatcor intellizent devia redresser les tiges le plus tôt possible et accumuler lit terre autour en la comprimant avec le pied; la racine se raffermit alor's et la plante souffre peu de cet accident.

\section{Maludies du muïs.}

Faut-il, aree Tillet (1), n'admettre qu'unc seule espece de maladie du maïs sous le som de charbon, ou bien avec Imhoft, De Candolle, ou l'auteur de l'article Maïs du Nouvecuu Cours dagriculture, en admettre deux, trois ou un plus grand nombre d'espéces? Je crois que l'on doit garder ici un terme moyen, et sans adopter toutes les maladies

(1) Tilles, Wém.ac. desse, 1760, p. $25 \%$ '. 


\section{(6o)}

que nous indique Imhoff ( $\mathrm{r}$ ), telles que l'ergot, le charbon, la carie, le rachitis et la rouille, on doit, selon moi, reconnaitre trois espèces de maladies du maïs :

L'une, le charbon, qui affecte seulement les fleurs mâles de la plante.

L'autre, l'uredo maydis (Dec., Fl. fr., $\left.\mathbf{n}^{\circ} .615, a\right)$, qui affecte les grains ou la tige dı maïs.

Enfin le rachitis, qui affecte l'épi.

Le charbon, que les Piémontais appellent vulgairement gavas, se montre dans les temps humides; il attaque principalement les glumes des épis mâles, les transforme en de petites tumeurs remplies d'une poussière noiràtre, et fait avorter les épis en empêchant la fécondation. L'urédo, que quelques auteur's, entr'autres Seringe (2), croient aussi le produit de

(1) Zea maydis morbus ad ustilaginem vulgò relatus Franc.-Jacobo Imhoff. Argentorati, ${ }^{7} 84$, infol., fig.

(2) Monographie des céréales de la Suisse, par Nicolas-Charles Seringe. Berne, 1818 , in- $8^{\circ}$, p. 199. 
l'homidité, me parait treancoup plutòt ère causé par la piquire d'un insecte. Tel est aussi l'avis de Ch. Bonnet ( 1 ) et de Duhamel.

En effet, si l'on consulte le rapport de la commission d'expérience nommée par la Société d'ạrriculture de Seine-et-0ise (2), l'on y lit ce qui suit: "On observa, en l'an XI, sur plusieurs tiges de maïs, des grains charbonneux. Quelques personnes présumèrent que les fréquens mouillaģes nécessitís par la sécheresse extraordinaire de l'année en poirraient être la cause. Pour vérifier cette assertion on a semé, cette année, deux petits carrés du même maïs, dont l'un fut mouillé chaque jour et l'autre abandomé à la nature; ces de'ux earrés d'épreuve n'ont présenté d'autre différence que celle de force et de grandeur dans les plantes qui avaient éti arrosées. Ce fut, au contraire, sur des pieds de maïs semés dans le grand champ que l'on ob-

(1) Recherchess sur l'usuge des fenilles dans les plantes, p. 329. Gottingue et Leyde, 1754 , in- $4^{\circ}$.

(2) Mém. de la Soc. d'agr. et des arts de Seine-etOise, t. II, p. 15. 


\section{(62)}

serva le long des tiges des excroissances charbonneuses."

Ces tumeurs, suite de piqûres d'insectes, se remarquent assez souvent sur le chêne et le rosier, pour que je puisse les supposer ici ayant la même eause. Quant à leur composition, je crois, sans cependant pouvoir encore en donner maintenant aucune preuve certaine, que le suc très abondant de cette plarite, s'épanchant au dehors par la piqûre faite à la tigge ou au grain, subit une décomposition particulière sous l'influence de la chaleur et de l'humidité auxquelles il est alternativement exposé.

Je me propose de faire, plus tard, quelques expériences à ce sujet.

$D^{\circ}$ après Parmentier ( $\mathrm{I}$ ), cet accident est d'autant plus commun que la plante est semée plus dru, qu'elle se trouve dans un meilleur fonds, qu'elle est plus abritée, et que sa tige est plus vigoureuse.

Quoi qu'il en soit des distinctions purement scientifiques que j’ai cherché à établir,

(I) Ourrage cité, p. 54 . 


\section{6i)}

La maladie que jappello mredo altaque tantó la lige à l'aisselle des fenilles, tantót les grains mêmes du maïs. Ia partie attaquéc grossit et prend la forme d'ume tumenr d'abord charnme, puis entierement remplie d'une poussière noiràtre, inodore et très ahondante.

Ces tumeurs ont depuis la grosseur d'un pois ou d'une noisette jusqu'à celle du poing. Lorsqu'elles sont parremes à maturité, l'épiderme qui les reconvait se rompt an moindre choc et laisse échapper la poussière. Cette maladie est donc intermédiaire entre le charbon et la carie. Sa poussière, comme celle dı charbon, est inodore et composée de glohules fort petits; comme celle de la carie, elle nait à l'intérieur des grains, pour se répandre ensuite en dehors. Imhoff (1) dit que ces tumeurs atteignent la grrosseur d'un ouf de poule de médiocre grosseur, mais que très peu sont plus grosses.

Leurs formes sont très variées; elles sont globuleuses, pyriformes, conoüles, cylindri-

(1) Ourrage cili, p. ?. 
ques, bossuées, etc.; la poussière noiràtre contenue dans ces tumeurs n'a, au reste, rien de contagieux, comme l'assurent Tillet (1), Imhoff et Parmentier, d'après des expériences nombreuses. M. le docteur Roulin a lu, le 20 juin r 829 , à l'Académie des sciences de Paris, un mémoire intitulé : De l'ergot du maïs, et de ses effets sur l'homme et les animaux. Il a observé cette maladie du maïs pendant son séjour en Amérique, et il a remarqué que les symptômes auxquels elle donne naissance ressemblent, sous certains rapports, à ceux que produit le seighle ergoté; ils en diffèrent sensiblement en quelques autres.

En Colombie, on donne à ce maïs le nom de peladero, c'est à dire qui cause la pelade. Il fait, en effet, tomber les cheveux des hommes qui en mangent; quelquefois, mais plus rarement, il cause aussi la chute des dents. L'auteur ne l'a jamais vu produire la gangrrène des membres ni des maladies convulsives. Dans les porcs qui se nourrissent de

(1) Mém. ac. sc., ı g6o, p. 88, in- $4^{\circ}$. 


\section{(ij)}

mais aygote, les poils tombent en quelpues jours, phos tard les membres de domion s'anophient ef se sontemnent à peine. M. Roulin n’a ju olserver los efleds de l'usage prolongé du grain altéré, parce que, sitót que le pore maigrit, il est sacrifić.

Les mules mangent sans difliculté le maïs ergoté, et l'on ne tarde pas à voir cliez elles la chute des poils, l'engorgement des pieds, quelpurefois mème la chute dı sabot: comme alors l'animal n'est plus propre à servir, on le relégrue daus des pàturages éloigunés des halitations; la sauté s'y rétablit, parce que la cause de la maladie ne subsiste plus, et le pied se recouvre d'une corne nouvelle.

Les poules nourries de maïs ergoté pondent fréquemment des neufs sams coquille. C'est une espece d'arortement qui n'a pas donné i l'auf le temps de se recourril de son enveloppe terreuse. L’ergot de maïs n'agit jamais avec plus de force que lorsqu'il est fraîchement récolti; le poison parait encore plus actif avant que le grain ait acquis sa parfaite maturité. Le mais, depnis qu'il a commencé i entrer en épis jusquan moment de sa ré- 
colte, est environné de nombreux ennemis. Les mammifères et les oiseaux s'en montrent également avides, et la surveillance la plus active peut seulc les en tenir écartés. Lorsque la récolte est gầtée par l'ergot, on se relâche ordinairement d'une garde fatigaute : alors, de jour et de muit, les animaux viennent pour se gorger de ce mauvais grain, qui agit sur eux avec la plus effrayante rapidité; il n'est pas rare de voir des singes, des perroquets tomber comme ivres au milieu des champs, sans pouvoir jamais se relever. Des chiens indigènes, des cerfs, qui sont également friands de maïs, mais qui ne viennent s'en repaitre que dans l'obscurité, éprouvent quelquefois le même sort.

"Après ce que je viens de rapporter, croirait-on, poursuit M. Roulin, qu'un grain eapable de causer subitement la mort puisse perdre en quelque temps ses propriétés délétères et devenir susceptible de servir d'aliment? c'est pourtant ce qui me semble prouvé. Nombre de gens dignes de foi m’ont assuré que lorsque le maïs peladero a passé les $\mathbf{P a}-$ ramos, hautes montagnes où règne un froid 


\section{( 67 )}

éternel, il se trouve déponillé de tontes qualités muisibles : ce qu’il y a de certain, c'ost quion porte asse\% souvent ce grain dans le village de la Cordillière, simé sur le versant opposé, et lit il est acheté par des hommes qui n’ignorent pas le dangerer qu'il y amrait a s'en servir dans le lien ou il a été récolté. M. Roulin fait remarquer, en terminant, que la température influe beancoup sur la proportion des prineipes immádiats qui entrent dans la composition du mais; c'est ee dont il a en occasion de s'assurer pour la tige de cette graminée. Dans les pays froids, comme Bahota, la tige de maïs n’a qu’une saveur très insipide; mais à Maraquita, où la chaleur est très forte, cette mème tigge est sucrée et fournit en abondance un sirop agréable ( 1 ). "

Cette poussic̀re charbonneuse n'est d'aucun effet sur l'économie animale. Imhoff a pris diverses doses de cette poudre étant à jeun; il en a pris aussi en gunise de tabae sans en avoir été incommodé, sans avoir renarqué dans ses

(1) Recueil de méd. vétérinairc, Vle année, $N \circ 68$, ¿. V1, août เ 829 , in-8", p. 468 et suiv. 


\section{(68)}

sécrétions rien d'extraordinaire, sans avoit senti d'irritation à la membrane pituitaire.

On trouve des figures bien faites de ces tumeurs dans l'ourrage d'Imhoff, dans celui de Ch. Bonnet, à la figure ro de la page 3r, et aussi à la fin de l'Instruction du professeur Buniva (1).

\section{Du rachitis.}

Cette maladie, plus commune que les deux premières, consiste đans un défaut de déreloppement de l'épi. Quelques grains seulement sur quelques centaines arrivent à leur grosseur ordinaire. On concoit quel serait le dommage qu'éprouverait un agriculteur qui aurait beancoup d'épis semblables dans sa récolte. La quantité du produit, d'ailleurs fort inférieur en qualité, serait considèrablement diminuée. Cette maladie dépend soụvent de

(I) Instruction pratique sur la culture et l'emploi du maïs quarantain en Piémont, insérée dans le t. IX des Mémoires de la Sociélé d'agriculuure de 'T'urin. Turin, 1812 , in-8。. 
re que le maïs a été semé dans un torrain topp paurre, et aturuel on 11 a pas dommc la quantite d'engratis sullisante, on encore de ce que le grain planti un peu tard a été expose a une température constamment froide et hormide, conme cela est arrive i plusienrs pieds de ma plantation, en is3o : pent-ćtre cette espece d'arrêt dans le developpement vient-ello du manais choix que lon a fait du grain deslimé aux semailles.

\section{Remède.}

Ces maladies ne font pas ordinairement de grands ravages daus les champs de maïs, surtont dans les années où la récolte est pen abondante. Le seul soin que l'on ait ì prendere, éest d'enlever les llemrs intiles lorsqu'elles sont malides, ou ces tmmeurs lorspu efles commencent a fasaitre; car satus cela, allirant it elles tout le suc de la plame, elles empécheraient le grain d'arriver à maturité.

Des animuatux qui altaqueme le mais.

Celle plante, qui est si recherche par los 


\section{$(70)$}

animaux que l'on élève dans les fermes, comme nous le démontrerons plus tard, est exposée, depuis son état de graine jusqu'à sa parfaite maturité, après avoir passé par tous les deggrés de développement nécessaires, aux ravages d'une foule d'animaux qui semblent vouloir disputer à l'homme une nourriture qui lui serait si utile s'il savait profiter de toutes les ressources qu'elle lui offre.

Lorsqu'on le sème, les merles, les grrives, les pies, les corneilles, les corbeaux, les pigeons, et, en Amérique, les perroquets et les singes, le recherchent avee avidité et en dévorent une grande quantité, si l'on n'a pas le soin de bien recourrir le grain de terre.

Il n'est pas inutile de rapporter ici commeut les indigèènes parviennent à prendre les singes, animaux si rusés ordinairement. Ils prouvent, en donnant presque constamment dans les piéges qu'on leur tend, que la gourmandise l'emporte encore sur leur instinct naturel.

Les habitans évident des noix de cocotier, praticuent une petite ouverture ronde daus la cog̣inille, et remplissent celles-ci de fruits de 


\section{(71)}

diverses espéces, entr'autres de mails, dout ces animaux sont très friands. Les noix sont ensuite répandues sous les arbres; les singes, apres les avoir flairées et retournées en tous sens, fourrent la patte (on pourrat dire la main) dans l'ouverture et la remplissent de butin; elle se trouve alors trop grosse pour être retirée, et l'animal est assez stupide pour rester ainsi empêtré, poussant de grands cris, mais sans songer à làcher prise et ne pouvant pas entrainer la lourde noix pour se sauves. On en prend ainsi un grand nombre.

Lorsque le maïs se déreloppe, il a surtout à craindre l'attaque des taupes, des mulots, etc. ;

Des fourmis, lorsqu'elles sont en grandes quantitis ;

Du grillus, grillo tulpa, qui coupe les racines lorsqu'elles sont encore tendres;

De la nitidula atrata, de Latreille, qui y cause des dommages considérables;

De la larve du hanneton (melolonta vulgaris), qui ronge aussi ses racines : ces larves se nomment rulgairement vers hlancs, 


\section{$(72)$}

mans, turcs, mulots, clievrettes; c'est probablement l'aire du Béarn, dont parle Rozier (1);

De la phalcena forficalis, ou pyralis forficalis de Viller, qui dépose ses oufs dans les tiges : ces larves, qui en naissent, en rongent l'intérieur et font périr la plante ou l'affaiblissent au moins de manière à nuire an développement parfait des épis;

Une partie de ces insectes sont figgurés dans une planche annexée à I' Instruction de M. Buniva (2);

Enfin, la noctua zere de M. Duponchel (3), insecte connu depuis peu de temps, et sur lequel je donnerai la description particulière de l'auteur, parait aussi faire beancoup de

(1) Cours complet d'agriculture, t. VI, P. 364. Paris, 1785 , in- $4^{\circ}$.

(2) Ouvrage citc', inséré dans le t. IX des Mómoires de la Société d'agriculture de Turin. Turin, 1812 , in- $S^{\circ}$.

(3) Histoire naturelle des Lépidoptères, par Godart. continuée par Duponchel, i 827 , t. IV, p. 363, pl. CXXII, fig. 4 . 
tort il celle plante. On ne possede encore yur lindividu male.

"Envergure, 15 à ic lignes.

"Les ailes supríriemres sont, en dessus, d'un " gris rougeàtre luisint, avec les nervures " noiritres el sampondries de gris, mon point

") central blanc et une ligne tunsverse ondu" lée et noiràtre, placée à çgrale distance de ") ce point et de la frange, qui est simple ct " de la mème comleur que le fond de l'aile. "Le dessus des ailes inférienres est blanc, ! " compris la frange, qui est sciparée du bord " extérieur seulement par une ligne de points " noiritres. Le dessous des quatre ailes est " egalement blane, mais légérement saupon"dré de gris vers l'extrénité des supérieures, " avec leur hord excéreur séjarce de la frange " par me ligne de protits points noirs. "Lat terce le corselet sont du mème gris $"$ que les ailes supériemres; l'ablomen est. " d'un gris plus pile. Los antemes sont gri") ses et liliformes dans les demx sexes, qui "ne diffèrent entrenx que par la forme de "l'abrlomen. Cenre espece, ajjonc M. Dupon"chel, que nons narous pu rapposter à an- 


\section{$(74)$}

" eune de celles décrites ou figurées dans les

" auteurs, nous a été communiquée par M. le

") capitaine de Villiers, qui nous a assuré

n qu'elle est très commune aux environs de

"Montpellier dans les champs de maïs, pour

" qui elle est. un véritable fléau lorsque sa

n) chenille vient à se multiplier, attendu que

" celle-ci se loge entre les feuilles qui enve-

" loppent l'épi femelle, aux dépens duquel

") elle se nourrit, s'y. change en chrysalide,

" et n'en sort que sous l'état d'insecte par-

" fait. M. de Villier's m'a ajouté quie son frère

") élevait cette chenille tous les ans sans pren-

" dre d'autre peine que de cueillir et renfer-

"mer dans une boite les épis qui en étaient.

") attaqués. Malheureusement il n'a pu m'en

") domner la description; mais, d'après sa ma-

J) nière de vivre, nous pensons qu'elle doit

") être glabre et ressembler, pour la forme,

" à celle du genre monagria de Treitschke,

" ou bien à celle du genre leucania dı même

" auteur, quoique son papillon ait quelque

1) ressemblance avec les noetuelles albi puncta?

1) et lithargyria. "

Lorsque le grain est rentré dans le gre- 


\section{$(75)$}

nier, il y a encore d'autres animanx qui lui font la guerre : ainsi, les rats, les somris, la ralandra stranaria (espree de charaneon), qui y fait quelquefois de très grands latrages, et anssi la teigne des lilés, du genre alucite.

On parvient toujours, aree les appareils de Cadet de laux et de Terrasse des Billons, à détruire cette chenille. Si la tẹnpérature n'excèrle pas 60 degrés, le grain conserve sa faculté grerminative.

J'ai trouve daus du maïs lin, apporté de Bourbon, une larve hexapode, vivaute, longue de 5 ou 4 liggnes, ayant l'extrémité cornće, le corps blanchàtre, toutes les parties latérales garnies de poils un peu distans; les mandilules etaient tris fortes. Cette larve appartiout a la classe des coléptires, et se rapproche asse\% des ténébrions. Je n'ai pu en decouvrir qu'un seul individu, malgyré mes recherches longues et minuticuses; je l'ai conservie pendant deux mois vivante an milieu du grain cassé dans lequel je l'ai trourée, et à l'époque du terme du coneours, li 15 jamvier 1850 , je l'ai enroyé a l'Acadinie. Le 15 septembre, 


\section{$(76)$}

on m'a rendu la boite qui la contenait; mais elle était morte. Cette larve, que j'ai montrée à quelques naturalistes célèbres de Paris, leur est inconnue; j’ignore encore à quelle espèce on pourrait la rapporter. Quant à son origine, elle est aussi fort douteuse : a-t-elle été produite à l'Ile-Bourbon, ou bien est-ce seulement depuis l'arrivée du báliot sur les côtes de France qu' clle s'y sera introduite? Cette double question, le temps seul pourra la résoudre.

Amoreux, dans son Mémoire (1), parle de sauterelles vertes qu'il a remarquées; mais il n’indique pas les dégâts qu'elles y font.

Fernandez, dans son Ornithologie du Mexique, décrit an chapitre LXXVII (2), sous le titre de Miacatololl sen ave germinis maizii, un oisean fort petit, qui a l'habitude de se poser sur les tiges de maïs.

(1) Supplément uu Mémoire de Parmentice sur le maïs, par le comte François de Neufchâteau, p. I 28.

(2) Dictionnaire des scicnces naturclles, t. XXVIII, p. 113 . Levrauli. 


\section{$(77)$ \\ Culture.}

Si l'on recherche pourquoi la culure du maiss est si vivement recommandée depuis quelques ammés, c'est que ce vigrétal est moins exposí que les atutres à une perte torale, parce que, dir Parmentier, page sof, " il ne demeure sur pried que pendant la plus " belle saison; qu'ilane sc plante qu'après les "getées; que son fruit, toujours enveloprú " de feuilles on ćcailles appliquées immódia" tement les unes sur le's autres, est garanti "de l'action de l'air, de l'humidité, de l'ar"deur du soleil et de la voracité des ani" manx destructeurs, en sorte qu'il croit el. " mùrit presque sans dingुger. "

Le comte Chabrol de Volvic, dans son ouvrigge sur le département de Montenotte, dit ce qui snit (1): "Dans le département, sur "le revers septentrional des Apennins, la "terre, malgyre les soins des labonreurs, se

(1) Statistique du département de Montcnotle, pas le comte Chabrol de Volvie, t. XI, p. 126 et i 3 g. 


\section{$(78)$}

" refuse généralement à produire en abon-

» dance du blé et du grain, le maïs en tient

" lieu : il réussit bien et est moins sujet aux

" accidens et à la grêle. "

"Le maïs, ajoute-t-il, est moins exposé "que le blé aux "maladies dangereuses, et

" il résiste mieux à l'atteinte des vents, de

"la pluie et de la grêle. "

Il serait curieux de syivre ces observations faites par un homme instruit, et d'examiner si les champs de mais sont moins souvent frappés de la grièle que les champs de blés, seigle, etc.

Cette plante précieuse a cependant été en butte aux attaques de quelques détracteurs. (Les choses les plus utiles n'ont-elles pas leurs ennemis?) On a été jusqu'à dire que c'était un poison pour l'homme et les animaux, et demander au Gouvernement d'en proscrire sa culture.

Des terres propres au mä̈s.

Toute terre, pourvu qu'elle soit riche, profonde, bien travaillée et suffisamment amen- 


\section{(7y)}

dée, convient an maïs; cependant il réussit mieux dans eolle qui est lérgere et humide que dans les antres.

M. Ic marquis de Beammont dil que le maïs aime me terre fraiche, mais non pas froide, ni trop momillée: l'excés d'hunidité le fait jamnir et empèche sa fécondation, et l'excès contraire produit le mème effet. II pense que le terrain le plus arantageusement situé est celui qui se trouve à / pieds environ au dessus des eaux souterraines.

"Je l'ai vu planter en Caroline dans des " sables presque purs, sur les bords de la ") Saòne, dans des argiles très compactes, " aux environs de la Corogne, dans des fis") sures de rochers schisteux et graniteux, et. ") dans tons ces lieux donner de copieuses ré" coltes (I)."

Les Basques ont réussi à le naturaliser dans le sol pierreux qu'ils habitent. Les terres sablonneuses sont celles qui conriemment le

(1) Nouvean Cours complet d'agriculuere, t. IX, ए. $29^{5}$. 
mienx : il y est d'mne bien meilleure quatité, mais en plus petite quantité.

Une terre trop grasse produit une tige robuste, des feuilles longues et larges, beaucoup de branches et peu d'épis, qui contiennent en petite quantité des grains très souvent colorés en rouge, et par conséquent dequalité médioere ou inférieure. Les terresargileuses conviennent peu à la culture du maïs; elles sont trop froides, trop humides ou trop sèches. Ces sortes de terres conservent trop long-temps, au printemps, le froid et l'humidité de l'hiver, et lorsqu'elles ont été réchauffées par le soleil, elles deviennent sèches et arides ( $\mathrm{I}$ ).

Kalm dit qu'une terre trop grasse le fait luxurier, expression laconique qui rend bien l'idée de l'auteur. Il réussit dans les sables des Landes, “ dans les terres de trans" port, des vallées de l'Ariége et de la Ga"ronne, et ne rebute pas les terrains pier" reux du Béarn. Je l'ai retrouvé, dit M. De "Candolle, jusque dans les hautes mon-

(1) Della collivazione del maiz del padre Gaetano Barasti di Buda, p. 36 et 37 . Vicenza, 1 788 , in-8. 


$$
5,)
$$

") Lagnes du Béarn, notamment près de Les" euns, a mue hauteur que je ne puis mesu"rer exactement, mais que je ne puis es"timer à moins de 1,000 mètres. Si l'on "part du calcul admis généralement, que ") 200 metres de hauteur verticale coniva"lent, pour la température, à mn degré " de latimde, on trouve que le mais doit ") rencontrer à Leesems a pen près la méne. " temperature moyeme que s il croissait à „5 degrés plus au nord : or, c’est précisé" ment le point septentrional oit il est cultivé ") en grand (I). "

Le maïs pousse très bien dans les forêts quion vient d'abattre et ou le blé ne réussit pas, à canse de la nomriture surabondante qu'il y trouve, laquelle le fait verser et ensuite pourrir (2).

On roit en Amerique des terrains si sees et

(1) Mémoires de la Société d'agriculture de la Seine, t. XI, p. 6o. Rapport du Voyage de M. De Candolle dans les départemens du suil-oucst de la France.

(2) Parmentier, ourrage cité, p. 65, note de M. Vassali. 
si maigres, qu'ils semblent incapables de produire, ils portent de très beaux maïs.

Suivant Arthur Young ( 1 ), la ligne de démarcation entre le pays au maïs et celui où il n'y en a pas est assez singulière. Elle commence à l'ouest du royaume, en passant par Saintes, Ruffec, Givray, Chàteauroux, Bourges, Pouilly et entre Nancy et Lunéville, et se termine au sud par une autre ligne passant à Mirepoix, Lodève, Vivier, Grenoble. " Cette liggne de démarcation, dit-il, peut " être regardée comme la division entre la " bonne agriculture dı midi et la mauvaise " agriculture du nord du royaume. Les sols "fertiles restent un an en jachères, jusqu’à "l'endroit où l'on cultive le maïs, mais ja" mais après cette liggne. "

L'opinion d'Arthur Young sur la liggne que, suivant lui, ne dépassait pas, lors de son voyage en France, la culture du maïs ne tientelle pas à ce que cette culture n'était encore

(1) Voyage en France pendant les années 1787,88 , 89, 9o, traduit de l'anglais par F. S., t. II, p. 191, $2^{\mathrm{e}}$ édition. Paris, 1794 , in- $8^{\circ}$, avec cartes. 


\section{( 85$)$}

que pen connue de la plupare des culcivateurs, puisqu’a cene époque ancun onvage sur cette plante interessanfe n'elat eneore tombe dans leurs mains, et qu'on ne leur avait pas démontré les avintageres de colle céréale sur beancoup d'antres anxquelles ils donnaient les plus grands soins, quoique d'un rapport bien inférieur? Et comme le pense an reste M. Lelieur, Arthur Young avait-il asse\% distingué, ou mème connaissait-il assez les différentes variétés hàtives qui peuvent réussir au nord?

En France, on culcive le maïs en grand dans les départemens

De l'Ain (Bourg),

De l'Ariegge (Foix),

De l'Aude (Careassome),

De la Charente (Angoulème),

De la Corrèze (Tulle),

De la Corse (Ajaccio),

De la Dordogne (P'rigueux),

Du Gers (Aucli),

De la Gironde (Bordeaux),

De l'Isère (Grenoble),

De la Hante-Garonne (Toulouse), 


\section{(84)}

Du Jura (Lons-le-Saulnier),

Des Landes (Mont-de-Marsan),

Des Basses-Pyrénées (Tarbes),

Des Hautes-Pyrénées (Pau),

Des Pyrénées-Orientales (Perpignan),

Du Bas-Rhin (Colmar),

Du Haut-Rhin (Strasbourg),

De la Haute-Saône (Vesoul),

Du Tarn (Alby),

De Tarn-et-Garonne (Montauban ).

On le cultive encore, mais peut-être en moins grande quantité dans les départemens

De la Côte-d'Or (Dijon),

Du Doubs (Besanȩon),

De la Drôme (Valence),

Du Gard (Nismes),

De l'Hérault (Montpellier),

De l'Indre (Chàteauroux),

D'Indre-et-Loire (Tours),

De la Moselle (Metz),

Du Rhône (Lỵon),

De la Sarthe (le Mans),

De la Haute-Vienne (Limoges).

En Bourgogne, il est peu de cultivateurs qui n'en fassent maintenant presque moitié de leur 


\section{( 85$)$}

récolte. Les pays qui donnent daus ees contrées le meilleur grain sont Mailly, à I liene d'Auxonne (Còte-l'Or'), Champlôtre, I lieue d'Auxonne (Côte-d'()r), et Echenon, près Saint-Jean-de-Losne; en général, tous les environs d'Auxonne du côté de la Bourgogne.

Dans ces dernières années, plusieurs aggriculteurs, et entr'autres fer M. Andrien de Cheptainville, de Seine-et-Oise, à l'exemple de Parmentier, se livrèrent avec avantage ò cette culture dans les départemens voisins de Paris.

Plus de 50 arpens de mais ont été cultivés, dans l'anncée 1829 , avec le plus grand succès aux environs de Paris, dans les départemens de la Seine, de Seine-et-Oise, Seine-etMarne et de l'Oise. La Société d'Horticulture de Paris, dans sa séance publique dn 8 novembre ı 829 , a décerni les prix fondés par M. Bossange père pour cette culture.

La Société a fait l'hommage d'un jeton en or, comme témoignage de sa vire gratitude, à madame la duchesse de Berry, qui a fait cultiver en mais plusieurs ares de terre dans son domaine de Rosny.

V. le baron Lonis, qui en a cultives sar- 
pens dans son domaine de Petit-Brie (Seine-etOise), dirigé par M. Thomas Bertier, de Roville, a obtenu le premier prix pour la simplicité et lat perfection de ses binages à la houe à cheval, la beauté, la qualité et la quantité de ses produits.

Le deuxième prix a été décerné à madame la marquise de Nicolaï, pour la simplicité de sa culture dans sa propriété d'Osny (Seine-etOise), son économie et la maturité deson maïs.

M. le baron Ternaux, qui l'a cultive à SaintOuen-sur-Seine (Seine), amrait eu le troisième prix s’il n'eût fait partie dı Conseil d'administration de la Société.

Il a été domé à M. Godefroy fils, propriétaire et agriculteur à Villenenve-le-Roi (Oise), qui en a cultivé 3 arpens, pour le bon emploi qu'il a fait des tiges, des spathes et rafles de cette plante. Cet agronome a eu l'obligeance de m'envoyer à cette époque des épis magnifiques yui provenaient de sa récolte.

Des mentious honorables ont été faites :

I". Ve M. Euggène Laffitte, maire de Verrière (Seine-rt-()ise), pour l'économie de sa culture - 1 la beanta de ses épis; 


\section{$(87)$}

$2^{n}$. De M. Camille-Beaurais, des beryeries, pre's Ris (Seine-et-()ise), ponr ses binages;

$5^{\circ}$. De M. Péliggot, pour sal culture de pomme de terre interealée dans son maïs, faite i) Montmorency-Enghien (Seine-et-Oisc);

4". De II. Quest, proprictaire el cultivat. teur à Bruycres-le-Chitel, arrondissenent de Corbeil (Scine-et-(Oise), qui a produit les renscignemens sur sa belle culture trop tandiveunent; il a obtenu plus de vingt-cing mille liges.

'Toujours guidé par les mèmes sentimens de philantropic et voulant continuer les encouragremens qu'il avait déjì fait distribuer à ceux qui se livraient à la culture du maïs, M. Bossanģe avait déposé à la Société d'Horticulture le grand ouvrage de Redouté sur les Lilinu'ées, pour ètro décerné comme prix ì celui des concurens qui aurait obtenn en 1830 les meillurn's résultats, sous tous les rapports, sur 5 hectares de terre au moins plantés en maïs.

Quatorze concurrens se sout présentés, ce sollt :

1". Le Roi des linancais, à Villiers (Scine); 
$2^{\circ}$. MM. Godefroy fils, à Villeneuve-le-Roi (Seine-et-Oise) ;

$3^{\circ}$. Quest, à Bruỵères-le-Chàtel (idein);

$4^{\circ}$. Camille-Beauvais, aux Bergeries (id.);

$5^{\circ}$. Alfred-Bosquillon, à Jarcy (idem);

$6^{\circ}$. Beaudoin, à Noisy (idem);

$7^{\circ}$. Bourgeois, ancien directeur du domaine royal de Rambouillet, aux Roseaux (id.); $8^{\circ}$. Achille-Vigier, à Grand-Vaux (idem); $9^{\circ}$. Baron Barre, à Courbevoie (Seine); $\mathrm{r}^{\circ}$. Benoist, à Livry (Seine-et-Oise);

$11^{\circ}$. Gérard de Villesaison (Cher);

$12^{\circ}$. Général Merlin, à Sillery (Seine-etMarne);

130. Deshaires, à Noisy (Seine-et-Oise).

$14^{\circ}$. Olherhart, à Farcy-les-Lys (Seine-etMarne).

Le département du Cher n'étant pas compris dans le concours, le nom de M. Gérard a dî être retranché.

La plupart des concurrens, après avoir lutté long-temps et arec persévérance contre les difficultés nombreuses qu'ils ont rencontrées, et principalement contre les intempéries des saisons très fréquentes en 1831 , ont été 


\section{$(8 y)$}

forcés de tenoncer à prétendre au prix. Iat Sociéte a offert à Sa Majesté Louis Philippe une médaille d'or, pour avoir tenté d'introduire la culture du maïs sous le climat de Paris. Le Roi avait fait cultiver, sous la direelion et par les soins de M. Jacques, a Villiers (Seine), i hectares on 11 arpens en une seule pièce divisée par des allées dins le pare du domaine.

Le grand prix a dé décorné à M. Godefroy fils, proprictaire et cultivateur à Villenewve-sur-Scine, pour ses belles cultures de maïs, reconmues les plus parfaites sous les rapports de l'íconomie dans l'exécution, de l'abondance daus les produits, et de l'utilité dans l'emploi. M. Godefroy a cultivé plus de 3 hectares en trois pieces.

La médailled'argent a été decernée à M. MIfred-Bosquillon, qui a obtenu des produits abondans qui ont laissé peu ì désires. Il a fait usage, avec habileté, d'instrumens ćconomiques et perfectionnés; il a cultive plus le 3 hectares en deux pièces.

Enfin, il a ete fait mention hounable de 11. Bourgeois, à Rambnuilles, pour les efforts 


\section{( $\left.9^{\circ}\right)$}

satisfaisans qu'il a faits. II a cultivé 3 hectares en deux pièces.

L'acclimatement possible du maïs est sans donte plus restreint que celui de la pomme de terre qui prospère partout, mais il y a encore de grandes parties de zone et des localités innombrables où on peut avec succès dépasser la liğne maģistrale d'Arthųr Young. Il est possible, dit un auteur, de cultiver le maïs partout ou le vin est tant soit pen généreux. On le cultive en Danemarck ( 1 ). Cette opinion est cependant contestée et surtout par Burger qui a fait une étude spéciale dı maïs (2). On le cultive dans la Carinthie et le nord du Tyrol, pays élevés et montaģneux couverts de neiģe, six mois de l'année. Voilà la preuve la plus convaincante que ceux-là se trompent

(1) Cours d'Agriculturc pratique, ou l'Agronome francais, par une société d'agronomes; rédigé par M. le baron Rougier de la Bergerie, 1. I, p. 336 , ${ }_{18} 8_{9}$, in-8 .

(2) Traité complet sur l'histoire nuturelle du maïs, par 1). Jean Burger. p. 79. Vienue, 18og, in-8 ( en allemand). 


\section{$\left(y^{l}\right)$}

grossierement qui šmagriment que ecte plante ne vient que dans les pays où croit la vigue, ou quil faille mime encore un phus grand degré de chalens, comme on en a l'opinion dans le nord de la linance a dais beancoup d'endroits de l'Aliemagne.

Le mais, yui descend jusqu'aux regrions les plus chandes de la zone rorride, ou les épis du froment, du soigrle et de l'orge ne panviennent pas a se dérelopper, introduit dans le nord de i'Europe, souflie du froid partont ou la temperature u'atteint pas $70 u 8$ degreés centigrades. Le climat de l'Alsace mème ne pent entièrement en assurer la récolte; on l'y cultive cependant en assez grande quantiti. On estime ¿t r,goo hectares l'étendue des terres qu'on lui consacre annuellement, et le jroduit i fo, 000 hectolitres. Le mais a sa place assignnée dans les assolemens; il a son comrs an marehés "t son débit est aussi certain que celui des autres cerciales (I).

Dans les départemens oi cente cullure nest.

(1) Annules asricoles de hosille. In liviaison. 1. 3 (x) 


\section{(92)}

pas encore établie, il sera toujours utile de faire des essais pour connaitre l'espèce qui conviendra le mieux aux lieux et au climat.

Quoique l'Angleterre soit située entre le $50^{n}$ et le $55^{\circ}$ degré latitude nord, son climat est très doux; on ne l'y cultive pas ou peu. William Cobbett n'a commencé à le cultiver en Angleterre qu'en r 826; des épis lui avaient été apportés de l'Artois par son fils.

Dans le Valais et quelques parties du canton de Vaud, on cultive surtout le grand maïs.

En Espagne, le maïs est surtout cultivé dans la Biscave.

A Bourbon, il se plait davantage vers la région moyemne de l'ile.

Le mais n'est presque point cultivé dans la Haute-Egypte; il l'est davantage dans la Basse-Égypte. On en fait assez généralement deux récoltes, tant de ce gुrain que du doura. L'une à peu près à liı même époque, savoir lorsque les canaux se remplissent et peuvent fournir, au moyen de puisards, l'arrosement nécessaire; la deuxième au printemps, et ce sont alors de rastes réservoirs ou puits, oì l'eau est tiréc par des roues à godets 


\section{$\left(9^{3}\right)$}

mues par des boufs, qui fournissent aux irrigations (1).

On le cultive peu dans l'Asie méridionale, et point du tout en Chine.

Le blé de T'urquie est au nombre des produits agricoles de Corfou; on le cultive dans les terres basses et situées an pied des montagnes. La vallée de Ropa en est couverte; il y végète d'une manière très favorable (2).

Les Bugres de Saint-Paul (Brésil) cultivent trois sortes de maïs : leblanc, le violet et me autre espéce nommée perurúca par les Paulistas.

Introduction de sa culture dans différens:

$$
\text { pays (3). }
$$

A. Gallo a été témoin oculaire de l'introduc-

(1) Considérations génćrales sur l'agriculture de l'Egypte, par Reynier, p. 69, in-8\%.

(2) Annales de l'agriculture française, par 'T'essier, t. XXI, P. 214. Extrait d'un rapport sur l'histoirs naturelle et médicale de l'ile de Corfou, par M. Paroletti.

(3) M. de Grégory la fait remonter an XIIIe siecle, d'après une indication trouvéce dans les archives de la cashédrale de Verceil. 


\section{$94)$}

tion de la culture du maïs en Italie vers le $X V^{e}$ siècle. Il raconte qu'on arait commencé à introduire cette plante dans la presqu'ile de Rovigo vers 1560 , c'est à dire soixante-huit ans après la découverte de l'Amérique, et que de là la culture s'en est répandue dans les Ėtats Vénitiens et la Lombardie (1). Cette assertion semble bien prouvée, car du temps de Mathiolus, qui écrivait en 57 r, le maïs était déjà connu en Italie et les paysans en faisaient déjà usage comme aliment.

Cette culture a commencé à Bellune et dans le Frioul, en r6ı. Le maïs était sur tous les marchés de la Lombardie et des États Vénitiens, et Venise en faisait déjà un commerce considérable (2).

On ig̣nore l'époque où on recommença à le cultiver en France.

Marea (3), dans son histoire du Béarn, dit

(1) Le venti Giornati dell' Agricoltora di Agost. Gallo, p. 534. $2^{\mathrm{e} e ́ d i t ., ~} 177^{5}$.

(2) Dell' Agricoltora, dell' Arti e Lettere di Antonio Zauon, 1 765-г 767, t. V, lett. 15.

(3) Histoire du Béarn, par Pierre Marca. 


\section{$(95)$}

qu'il étair comnu dans le midi de lit litance ver's lit fin dn $\mathrm{XV}^{\mathrm{c}}$ siecte.

Dans les gazetles d'agrliculture du 6 janvier 1769 , on voil qu'il a commencé à être cultivé dans le Maine vers 1736 ;

En Angonmois, depuis le commencement du XVII' siecle.

En Angleterre, on commenca déjà, en r 562, it fiare des essais pour sa culture ( 1 ); il parait quiils ont été peu suivis.

II a 'té importi en Allemagne par diverses voies et à différentes époques. La Carinthie apprit à comnaite cette plante de trés bome heure par les Italiens. Dans la vallée de Gail, yui n'est séparée de l'Italie que par la chaine des $\Delta$ lpes carnicyes, la culture du mais y esı indigène et pratiquée depuis un temps immémorial : de lì, elle s'est répandue, vers le milien du sircle passé, le long de la Drase ou Draw, dans la Carinthie inférieure, et ensuitu dans le reste du pays.

La Styrie et l'Autriche recurent le mais par la IIongrice la Croatic. En I 755, le com-

(1) Turners herbal, P. I 1, P. 58. 


\section{$\left(9^{6}\right)$}

merce du maïs était si répandu en Styrie, que Charles VI se vit forcé, pour empêcher les disputes sur les dixmes, de.créer une loi sur la dixme du maïs.

En Autriche, où l'on tient toujours à l'usage des jachères, cette nouvelle plante ne peut avoir un accès facile. En I682, lorsque le baron de Hohberg écrivait son grand ouvrage sur l'économie rurale, il n'y avait que les vignerons qui mettaient dur maïs sur les petits espaces non cultivés; ils suspendaient les épis de diffẻrentes couleurs comme ornement dans leurs habitations.

De l'Italie, il passa en Suisse, et le long du Rhin; il se répandit dans l'Alsace et le duché de Bade.

Les Portugais le portèrent de très bonne heure à la côte occidentale d'Afrique et dans les Indes ( $\mathrm{I}$ ).

M. le capitaine Bedfort, royageur anMais, vient de pénétrer récemment dans l'Assam à la base de l'Himmalaya, et il y

(1) Histoire générale des Voyages, par l'abbé Prérost, t. IV, p. 297. La Haye, 1747. 


\section{(97)}

a trouve (lindian corn) le mais en pleine cultire (1).

De la place du mais dans les assolemens.

Ce chapitre, fruit des olsservations d'un agriculteur distingué, M. Schwer\%, doit lixer l'attention du lecteur' (3).

"On regarde ici (Alsace) le mais comme " une mauvaise préparation pour le froment;

" aussi les cultivatenr's qui suivent l'assole" ment triennal ne le placent-ils qu'après la " céréale d'hiver, et le font-ils snivre par une " autre récolte-jachère, telle que le tabac ou les " féres. Jene me rappelle pas de l'avoirvu dans " aucun assolement à la place de la jachère, " comme préparation pour le blé, mais je l'ai " sourent vu précéder l'épeautre. Ceci ne s'ac" corde nullement arec ce que nous savons " du midi de la France, où l'on cultive alter" nativement et sans discontinuer du maïs ou

(1) De la Roquette, Histoire de l'Amćrique, notes du premier volume.

(2) Annales agricoles de Roville, Ve livraison, p. 306 . 


\section{$\left(9^{8}\right)$}

" du blé, ce qui, suivant Arthur Young, est le

" plus fort produit que la terre puisse fournir

" à la nourriture de hommes et des animaux.

"Mais il y a une différence totale entre les

" sols, et particulièrement entre les climats

" des deux pays. Là où l'on n'a pas de gelées à

" craindre pendant le mois de mai, et où, par

" conséquent, on peut semer le maïs un mois

" plus tôt que dans la partie septentrionale de

" l'Allemagne, et où en outre les chaleurs hâ-

" tent sa maturité, là ou a le temps de prépa-

" rer la terre pour le blé après la récolte du

" maïs. En Alsace, ceci n'est pas praticable.

"Ce n'est que là où l'on cultive le maïs dans

" des terres sablonneuses, et où, par consé-

" quent, on peut semer le mais très tard,

" qu'on fait suivre le maïs par ce dernier.

"Si le blé ne vient pas bien dansl'année qui

" suit immédiatement le mais, on a remar-

" qué qu'il vient très bien dans la deuxième

" année. Le maïs est une excellente récolte

" préparatoire pour le tabac, les fèves,

" et particulièrement pour le chanvre et

"l'orge, tellement qu'on peut compter sur

" deux septièmes en sus, quand cette céréale 


\section{(y)}

3) suit le mais, an lieu de sucéder an bli.

"Pourquoi les enltivatemrs qui suivent lesys-

" time triennal, el yni ont l'expirience decette

" vérite, n’abandon:ent-ils pas lenr misérable

"routine? La nature a beau les iustruire, il

" est érit dans le code barbare de ce systeme

"que les récoltes d'hiver suivent les récoltes"jacheres (1).

"Cette planite effrite en général le terrain.

(1) l'our comprendre ceci, il faut siroir queu Asace, aiusi que dans beaucoup d'autres cautous où le sol est fertile et où les engrais sout abondans, on a conservé l'assolement triennal, en substituant une récolte sarclée à la jachère. J'ai exposé ailleurs les motifs qui me fout regarder cesysteme de culture comme vicienx el comme moins profitable, dans presque tous les cas, que l'assolement triennal dans toute sa pureté, c'est it lire avec la jachire. Je pense qu'il fant ou adopter franclsement un assolement alcernes, ou s'en tenir à l'assolenent triemnal pur, parce que l'ou ne peut arriver dans un assolement de trois ans, dont deux sont occupés par des récoltes ele céréales, à ancume bonne combinaison sans l'intervention de la jachère; je veux lire ì ancume combinaison qui assure le nettoiement du sol. On peut voir ici que M. Schwerz est entièrement de mon avis. (Note de $M$. de Dombasle.) 


\section{$(100)$}

"Dans les plaines de Toulouse et des landes

" de Bordeaux, on fait suivre cette culture

" par une année de jachères. Dans les vallées

" des Pyrénées, où les engrais sont abondans,

" à cause de la multitude des bestiaux que

"fournissent les montagnes voisines, on n'a

" le plus souvent pas de jachères, et on alterne

„ entre le blé et le maïs.

"Le maïs vient très bien après la pomme " de terre. Comme cette plante ne se sème

" qu'à la fin d'avril ou au commencement de

") mai, il est toujours utile dé donner encore

" un labour à la terre au printemps. On don-

"nera d'abord un trait d'extirpateur en mars,

") on labourera en avril, le sol sera égalisé

" par un hiersage, et l'on tracera avec le

" rayonneur les lignes qui doivent recevoir

") les semences du maïs. Je place les liggnes à

" la distance de 27 pouces, et la semaille "s'exécute avec le semoir à brouette (I)." On le voit prospérer après une récolte de betteraves; il réussit succédant à des vesces

(1) Annales agricoles de Roville, III liviraison, 1829 , p. 51 . 
eoupées en vert, suivies d'un semis de navets; enfin, après des cultures de sainfoin, d'orge ed d'aroine; et la vigueur des tiges, ainsi que la grrosseur et le nombre des épis n'en sont pas altérés.

\section{Engrais.}

Les afrriculteur's sont fort peu d'accord sur le genre d'engrais qui convient le mieux au mais; mais, avant d'indiquer les différens engrais dont on se sert, je veux dire quelques mots surcenx que l'on emploie en Amérique.

Les Indiens pour animer les buttes de terre sur lesquelles le maïs est semé, y mettent deux ou trois poissons de l'espéce qu'ils appellent aloof ( $\mathrm{I}$ ): ce poisson engraisse et fertilise

(1) Le not aloof ne se trouve, it ma connaissance, dans aucun de nos voyageurs, dans aucun ourrage d'histoire naturelle. Seulement, d̀ la figure f́ de la planche VI de la prentère partie de Rénard, est représenté, sans description, le laouff, poisson de l'Archipel des Indes, probablement de la grande fanille des percoïdes, et très voisiı, en particulier, des esclaves (therapon), autant qu'on en pent jugger d'après une grossic̀e représentation. Il scrait possible qu'il se rapportit i l'espece citéce ici. (H. Cloquet.) 


$$
(\cdot 102)
$$

ce petit tertre au point de lui faire produire le double. Les Anglais ont goûté cette pratique des Indiens dans leurs établissemens, où le poisson ne coûte que le transport. Ils y emploient, avec un succès admirable, des têtes et des tripes de merluches. A Atica, à Atilipa, Villacori, Malla et Chilca (Pérou), on n'engraisse les terres qu'avec une prodigieuse quantité de têtes de sardines ( $\mathrm{I}$ ).

A New-Yorck, on se sert aussi, avec avantage, des entrailles et de la tête des morues et des aigrefins.

Voici, d'après Burger. (2), quels sont les engrais que l'on doit employer de préférence :

$r^{\circ}$. La poudrette, le meilleur de tous les fumiers végétaux et animaux employés pour la culture du maïs;

$2^{\prime \prime}$. Le fumier des bêtes à cornes consommé et laissé en repos pendant cinq mois pour les terrains légers et sablonneux;

$5^{\circ}$. Le fumier fiais, qui rend le labour plus

(1) De Pauw, Défense de la recherche philosophique sur les Américuins, 1. 71. Berlin, 1770, in-12.

(2) Ouvage cité (en allemand), p. 108 et suiv. 
diffrcile; on doit s'en servir, surnou dans les terrains lourds et argileux;

$4^{\circ}$. Le funier de cheval; il convient beaucoup moins, et comme il contient moins de parties ànimales, il faut en mettre beacoup' plus; il peut ètre employé dans les mèmes circonstances que le précédent ;

5\%. A défaut des fumiers précédens, on pent encore employer cenx de brebis, de eochons, de poules et de pigeons, qui peuvent ètre aussi avantageux que le fumier frais.

Lorsqu'on a peu de funier, il faut le porter dans les intervalles des endroits ou doit ètre semé le maïs; on peul alors le répandre sur chaque pied avee facilité et en épargner beancoup, parce que, lorsquion le répand également par tout le champ, il firorise anssi la végétation des mauvaises herbes.

La chanx, la marne, le gypse sont peu avantageux, comme engrais, pour la culture dı maïs.

En serait-il de même de la suic et du saug, 'jui n’ont pas été encore employés?

Au Pérou, on emploie arec modération et alors avec beaucoup d'atraurage, conuncengrais, dans la culume du mails, le guenen, substance 


\section{( 104$)$}

animale fossile composée, d'une part, de matières animales qui ont beaucoup de rapport avec la fiente des oiseaux, et, de l'autre, de sable en partie quartzeux, en partie ferrugineux. Il se trouve très abondamment dans la mer du Sud, aux îles de Chinche, près de Pisco, ainsi que sur les côtes et îlots plus méridionaux.

J'ai fait, dans l'année 1830 , un essai dont on pourrait peut-être, par analogie, tirer quelque parti. J'ai mis, au pied d'un maïs ayant déjà 4 pouces de hauteur, des grains de cacis qui étaient restés pendant une année dans de l'eau de vie, et que l'on renait de presser pour en obtenir la liqueur bien connue, dite de cacis. Cette masse humide, et qui pesait à peu près 4 ou 5 livres, fut recouverte d'un peu de terre. La jeune plante ne tarda pas à croitre arec rigueur; sa tige devint un tiers plus forte et plus haute que celle des maïs voisins, dont la semaille provenait du même épi; les deux pieds étaient plantés dans la même terre engraissée arec le mème fumier, à la même exposition, et séparés seulement de 5 ou 4 pieds du mais qui faisait l'objet de notre essai. L'épi devint aussi beaucoup plus fort et mùrit plus promptement. 


\section{$(105)$}

J'engrage les cultivateurs à essayer le mare provenant dela fabrientiondu vion, du cidre, cte.

On pourrait sans doute bruler les tiges sur le champ mème, et firire servir une première récolte pour en obtenir une seconde plus avantagense. Hlarasti di Buda (r) est d'un avis contraire; il pense quion devait brùler les tiges on racines hors du champ, el y répandre ensuite les cendres.

Dans le muncro du mois de juillet 1758 , du Journal économique, on doune le conseil de couper les tiges et de les mettre sur la terre labourée qui est dans l'entre-deux des sillons. On observera néanmoins de ne mettre les còtes des deux rangées que d'un côté et sur une seule planche, en laissant l'antre vide.

Ces tigres, rangres sur le champ comme on vient de le dire, laisseront à la charrue la liberté de lahourer les mottes ou plates-bandes de terre gui contiement encore les troncons des liges.

$\Lambda$ mesure que la charrue dans le labour arrachera on déterrera ces troncons, it y aura

(1) Della collivazione dell' maiz, elc., p. 76 , ill-8o. Ouvage cité. 


\section{$(106)$}

des hommes qui tireront avec des crocs toutes ces souches, pour les mettre sur la planche vide qui sera à côté, en observant de bien secouer la terre qu'elles auront à leurs racines.

Quand on aura àrraché ces souches dans tonte l'étendue du champ, on les y laissera, pendant quelques jours, sécher à l'air et au soleil ; après quoi, on les ramassera par petits tas de 6 pieds en 6 pieds dans le fond des plates-bandes, et on y mettra le feu dans le champ même. Les cendres qui en proviendront seront éparpillées avec soin sur la partie de la terre qui aura produit ce grain, et qui, par ce moyen, se trouvera purgée de tous les vestiges du blé de Turquie. Après que les souches seront brủlées, il faudra domer deux traits de charrue, l'un à droite et l'autre à zauche du fond des plates-bandes, pour les combler avec la terre que l'oreille de la charrue y jetiera; ensuite on labourera tout le champ en travers, et on le hersera bien. II sera alors en état d'être eusemencé en froment ou en autre cérẻale, comme s'il n'avait jamais produit de blé de Turquie.

Quelques cultivateurs séparent la tige des atcines et laissent celles - ci pourrir sur le 


\section{(107)}

rhatnp; dhes servent ainsi d'engratis pour l'allnce snivante. Si le maïs était placé après des pommes de terre fumes, il exigenat encore, dans la plupart des rireonstances, une denilimmure, ponr qu'on en obtimt une récolte complete sinns qure le sol restit trop épulisé pour les recoltes suivantes.

Les terres ne doivent pas être fumées beallcomp de temps avant les semailles, mais an moment du labour, (pui se fait avant l'ensemencentent, puisque, suivant les principes fondamentanx de l'aygriculture el d’aprés lir science naturelle, les semenees se developpent. et sortent de la terre sans le concoms des engrais, mais seulement à l'aide de la clableur ct de l'humidité. Il est suffisant que les germes des semences dévoloppées puissent commencer à profiter de la boune ef substantielle qualite de l'engrais aprés trois on quatre semalines, temps ou ils penvent tires cetterigneur ncicessaile à mo jeume plante.

Péparation du errain.

Nous arous parle de la qualité de la terre mecessaire at mäs; il faut maintenamt indi- 


$$
\text { ( } 108)
$$

quer comment on la prépare pour recevoir la semence. Ordinairement, en France, on donne deux labours successifs à la terre, en février et arril; quelquefois, en Alsace, on laboure plus tôt, et on en donne un troisième au printemps, avant d'y déposer la semence.

C'est après la récolte des fourrages qu'on laboure, en Italie, les champs avec la bêche (r); on commence vers le milieu d'avril, et on continue pendant tout le mois de mai; ce n'est jamais qu'après un labour à la bêche que l'on sème le blé de Turquie, qui doit faire la récolte de la troisième année (2).

On a fait l'expérience que, dans les terres sablonneuses sans consistance, les labours d'hiver sont nuisiblez au maïs : aussi laisset-on intactes (en Alsace), pendant l'hiver, les

(1) La bèche donve incontestablement le meilleur labour au mais; cependant une charrue qui peut prendre une forte entrée et dont le sillon reste profond, qui, par une double oreille ou par un versoir doublenent incliné, amenblit bien la terre, peut heureusement suppléer la bêche ou la houe, et à ce mode d'exécution se joint l'économie.

(2) 'Tableau de l'agriculture toscane, par Simonde de Genève, p. 8o, in-So. 
éteules de seigle dans lesquelles on compte mettre le maïs.

" L'expérience dans la culture du maïs " m’a prouré, dit Lelieur, qu’il ne veut point. " une terre défoncée; en effet, on le plante " sur le uait de la charrue; " et il ajoute : "Il est remar(juable que le maîs planté dans " les jardins pousse trop vigoureusement en " herbe aux dépens du grain. Le fonds que ") ses racines trourent prolonge la durée de la " vegétation, et pour cette raison il mùrit ") toujours plus tard que celui que l'on plante " en même temps dans les champs (I). "

On voit que l'opinion de Lelieur est différente de celle des autres agriculteurs; il fint done, pour se décider, faire attention à la nauure du sol destiné à cette culture.

\section{Des semailles.}

Le temps des semences est variable, suivant les pays. Ainsi, en France et surtout

(I) Annales de l'agriculture française, t. XXXII, Ire série, p. $25 \mathrm{r}$. 


\section{$(110)$}

dans les environs de Paris, on ne pent livrer le maïs à la terre avant la fin d'avril ou le commencement de mai, époque à laquelle on a rarement à craindre les gelées, qui pourraient faire perdre ou endommager beaucoup la récolte en faisant périr le germe encore tendre du grain au moment où il se développerait; en le semant plus tard, on risquerait de ne pas le voir arriver à maturité.

Si les terres destinées à recevoir le grain sont lourdes, grasses, argileuses, l'ensemencement devra se faire plus tard que si elles itaient légères et sablonneuses. Nous en avons dit plus lıaut la raison, lorsque nous avons indiqué les terres qui conviennent au mais.

Les Égyptiensle sèment en mars et avril (I).

Les Américains plantent le maïs depuis mars jusqu'en juin. Les Indiens sauvagyes, qui we connaissent rien de notre division d'année par mois, se guident, pour la semaille de cette plante, sur le temps où certains arbres de leurs contrées commencent à

(1) Description de l'Egypte, t. XXI, édition Panchoucke, in-8 . Flore de Delile. 
bourgeonuer, on sur la vente de cortains poissons dins lemrs rivieres (1).

En Italie, on peut le semer pendant les trois mois d'avril, mai et juin : on en sime mème quelquefois sur les champs qui viennent d'être moissonnés; mais alors e'est une espece particuliere qui produit moins et qui vient plus i ite.

A Vicence et à Padoue, situés sous le $45^{\circ}$ de. gré, on ensenrence déjì sur des champs situés ver's des collines, an milien de mars, et dans la plaine vers le commencenent d'avil.

A Ferrare, qui est undemi-degré plus ausuri, on commence au 16 mars, et celte opération est. toujours terminée vers les derniers jours d'arril.

Le blé de Turquie semé dans la plane, à la fin d'avril, se passe fort bien d'arrosemens et ne souffie pas de la sécheresse, pourvu qu'il $y$ ait des pluies an commencement de juillet, comme il arrive presque toujours (2).

Près Debreczin on Debretzin, ville de la

(1) Encyclopédie, article Maïs.

(2) T'ablécue de l'agrisuluere tossane, ourrage: cili. p. 79 . 
Hante-Hongrie, lequel endroit est cité comme très chaud, on fait la semaille vers la miavril ( $\mathrm{I})$.

Choix de la semence.

Le choix de la semence est bien essentiel pour obtenir de belles tiges et des récoltes avantageuses.

Les épis qui contiennent les grains destinés à l'ensemencement devront se récolter au milieu des campagnes les plus découvertes et bien dominées par le soleil; les grains devront être luisans, lourds, de belle couleur jaune ou blanche, sains, mûris sur le champ et sur leur tige, et non vieillis dans les greniers. Cependant il faudra les gुarder jusqu’à l'époque des semailles dans un endroit sec et aéré, de manière qu'ils ne puissent se rider même en hiver.

On doit préférer pour semence le maïs de la récolte précédente. On doit, autant que

(1) Die cultur und benubung dis turcischens maizens, oder mays, par Dr Jol. Christian Gothard. Erfurt, 1797 , in-12 (en allemand). 
possible, laisser les grains adherens ì lenr rafle, jusquia momeut.de les emplover. On doit ne prendre pour semence que les phus beaux épis et choisir cucore les grains les plus sains; ils sont ordinairement place's an milieu (1). Il fint en rejeter tous les grains piqués de noir on poreces de trous de vers, on doit les metre de róté jour nommir la volaille. Si l'épi est compose de grains de diffirentes coulems, chapue grain de meme conlemr doit ètre mis dans mon panjer à part; n'est que par ce moyen que l'on peut évilu. ces transformations toujours muisibles à la bonté du produit (2).

(1) M. Mathien de Dombasle, daus la Ve livinison des Annales agricoles de Roville, dil, p. 312, que cette méthode est assez généralement en usage dans les huit ou dix communes du département de la Meruthe ou la culture du maïs'est introduite.

(2) Telle est, sur la transformation de coulenr des grains de mais, l'opinion de Kalı. Marasti di Buda, p. 22. de son ouvrage, croit, an contrair, que celte transformation dépend du terrain, et qu'elle a plus souvent lieu dans les terres grasses que dans celles qui sont léperes. 


\section{(114)}

On doit bien prendre garde de mélanger les bonnes espèces qui deviennent presque toujours inférieures en qualité, exemple, le Turquie bâtard ou métis de Bourgogne. Il tire son origine de celui de Romanie, c'est à dire qu'en se servant de sa gyraine deux années de suite pour la semence, l'épi grossit et devient inférieur en qualité. Lorsqu'on veut conserver le Turquie de Romanie, il faut avoir le soin de choisir les mess ou épis ayant les plus petits grains et les plus serrés, et les changer chaque année.

Parmentier assure (page 74) que du grain de quatre ans non taché de moisissure, qu'on n'avait pas égrené immédiatement après la récolte et qui avait séché dans l'épi, lui a réussi presqu'aussi bien que du grain nouveau.

M. de Beaumont a semé du maïs récolté depuis sept ans, et ce maïs a aussi bien réussi que le nouvean; il a seulement levé un peu plus tard (1).

(1) Annales de la Société d'horticulture, t. V, p. 94. 


\section{Préservatifs de la semence.}

Avant de parter des preservatils de la semence, il serait utile de savoir si l'on peut, avec cerritude, préserver le grain des animaux qui allaquent le mais, en le faisant macéres plus ou moins long-temps dans une solution queleonque. Si l'on observe que rarement le yrain déposé en terre y est attaqué par les insectes avant de pouroir pousser sa tige, et quialors la plante se nourrit surtout par le suc qu'elle tire de la terre, au moyen de ses racines, on verra que si le maïs dépériı quelquefois au moment même où il est le. plus vigoureux, c'est parce que des larves d'insectes, comme celles du hanneton (melolonta vulgaris), dévorent non seulement le grain, mais les racines de la jeune plante. Je crois done les préservatifs de quelques anteurs seulement utiles à donner, pour ainsi dire, plus de force au germe. C'est ainsi que l'on peut employer l'ean provenaut dn fumier de bouf on de cheval; une légere solution alcaline faite avec des cendres de bois neuf, on de la 


\section{(116)}

chaux délitée depuis quelque temps. On cite encore comme très avantageuse une dissolution de nitre dans l'eau de pluie.

M. Morel de Vindé emploie une dissolution d'eau, vitriol bleu et sel de cuisine contre les maladies du blé : on pourrait l'essayer pour le maïs.

Ce procédé, qui consiste à tremper le grain plus ou moins de temps dans des solutions de natures diverses, fournit encore le moyen d'enlever les grains qui surnagent et qu'on ne doit pas employer pour les semailles, comme étant de mauvaise qualité.

\section{De l'ensemencement.}

Quel que soit le mode de semaille que l'on veuille adopter, mème celui dit à la volée, le maïs veut être planté par lignes espacées de 2 à 3 pieds de distance; la distance dans les lignnes veut être la même.

Dans la Haute-Garonne, on dirige autant que possible les rangées du levant au couchant.

Ponr l'enterrement des graines, in pouce dans les terres fortes et un pouce et demi dans 


\section{(117)}

les terres légeres, telle est la profondeur conrenable. Si l'on comrait trop la semence, elle germerait plus difficilement. 'Toutes les personnes qui ont étulic l'histoire naturelle s'accordent à penser que toutes les semences faites dans les terrains légers et sablonnenx doirent se faire plus profondément que dans ceux qui sont gras et argileux.

II y a sept manières de semer le maïs :

$1^{\circ}$. En suivant la charrue;

$2^{\circ}$. En faisant des sillons en travers;

$3^{\circ}$. A la volée;

$4^{\circ}$. A la hone;

50. Au plantoir, au moyen du cordeai;

$6^{\circ}$. Avec le semoir-maïs a hrouette;

$7^{\circ}$. Avec le semoir de Burger.

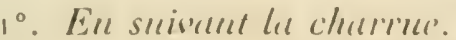

Ce procédé consiste à faire suivre celui qui conduit la charrue à oreille mobile par le semeur qui laisse tomber le grain de distance en distance. La mie gue l'instrument a tracée et qui recoit la semence est recouverte au retour de la charrie. 
II doit y avoir alternativement un sillon semé et l'autre qui ne l'est pas, de telle façon que les ranģs de maïs se trouvent espacés entr'eux d'environ 2 pieds, selon que le soe de la charrue est plus ou moins large.

Près d'Alby ('Tarn), immédiatement après cette opération, on aplanit la terre soulevée par la charrue, et l'on brise les mottes autant que possible, afin qu'elles n'étouffent pas le grain qu'elles recouvrent. Cette opération se fait avec un instrument en forme de marteau à long manche.

Le champ, après ce travail, n'est pas cependant absolument uni, mais laisse mieux voir le grain, à mesure qu'il germe.

\section{$2^{\circ}$. Sillons en travers.}

La manière de planter le blé d'Inde, pratiquée par les Anglais en Amérique, est de former des sillons égaux dans toute l'étendue d'un champ à environ 5 ou 6 pieds de distance, de labourer en travers d'autres sillons à la même distance et de semer le grain dans les endroits oì ils s'entrecroisent et se rencon- 
Irent. Its reconvent diun pouce de terre la semaille avee la beche, ou bien forment avec la charrue un autre sillon par derriere, qui renverse la terre par dessus.

Harasti di Buda ( I) indique $n$ instrumen! pour faire colte opéralion; il consiste en mm table forte et grosse de la longuenr de 4 ou 5 brasses et de la largenr de 4 ponces. On y fait avec unc vrille des trous a distance le.2 ou 3 pieds l'un de l'autre; on lixe dans chapue trou des dents de bois tres fort, grosses d'un ponce et longues de 5 , lespuelles doivent etre pointues par le bout; on adapte an long hout de la table nu manche longr et fort, de maniere que cet instrument ressemble beancoup à une herse. II se conduit en droite ligne par sillon dans la terre préparce pour l'ensemencement; il se traime en longueur, puis en laryeur, et de cette manière creuse à sonhait et forme également les huttes et creux désirés. Par celte méthode simple, on obtient un triple avantage : $1^{\circ}$. les lignes se forment de: suite; $2^{\circ}$. elles sont droites, étant à la mème

(1) Ouvrage cité, $p$ q́ et 42 . 


\section{$(120)$}

distance; $3^{\circ}$. elles sont d'une profondeur égale.

\section{$3^{\circ}$. A la volée.}

On sème le grain à la volée avec la main, comme on le pratique souvent pour le blé, et on le recouvre ensuite avec la charrue.

Cette manière de semer est peu employée, à moins que l'on ne veuille se procurer du fourrage. Alors, lorsque la plante a atteint 2 pieds de hauteur, on arrache les pieds intermédiaires, de manière, cependant, que les lignes soient toujours observées et que l'on puisse faire le travail nécessaire pour obtenir ensuite la récolte du grain.

Les pieds arrachés servent pour nourrir les bestiaux:

Quelle que soit l'attention que l'on porte en arrachant les pieds intermédiaires, les lignes ne sont jamais aussi régulières que celles faites par les autres procédés.

$$
4^{\circ} \text {. A la houe. }
$$

Ce mode d'ensemencement est encore usité 
dins quelepues pays. A l'lle-Bourbon, par exemple, on n'en emploie pas d'autre.

'Tel est anssi le procédé suivi en Mlsace, vit on emploie anssi quelquefois la binette.

Quand on vent planter le mais avec la houe, ee yui a lien dans des terres sablonneuses el mene asse sonvent dans des terres consistantes, voici la manière de se servir de et instrument. On ne herse pas le champ après le dernier labour, pour que l'ouvrier puisse se guider d'apres les traces de la charrue. Celuici suit une de ces traces sur laquelle il fait des trous; leur distance se mesure tout simplenent par la longueur qu'il peut atteindre avec sa hone, en placant le pied le plus en arrière sur la dernière touffe qu'il a plantée. De cette manicre, les distances en lignes seront d'm hon pas et celles des lignes entr'elles d'un pas et demi. Les trous sont creusés l'un apres l'autre à la profondeur de 5 ou 4 pouces, et l'on y met du fumier. Apres cela arrive le planteur, qui pousse arec le pied la terre on plutòt le sable des deux côtés sur le fumier, et place les grains de semence, non immidiatement an dessus dufumier, mais sur le bord, et encore 
avec le pied, il recouvre avec un peu de terre (I).

5o. Au plantoir, au moyen du cordeau.

Ce procédé, fort long, ne peut être suivi que daus de petites cultures. Il consiste à semer le maïs à la distance de 2 pieds, en faisant avec le plantoir des trous de 15 à i 8 lignes de profondeur, dans lesquels on met un ou deux grains que l'on recouvre de terre immédiatement. Cette méthode, la plus parfaite et aussi la plus dispendieuse, est la plus longue. Quelques peuples dont les connaissances sont peu étendues se servent d'un moyen analogue.

Les Mexicains font tremper la semence pendant un jour ou deux dans l'eau. Chaque membre de la famille en remplit de petits sacs, tous les pendent à leur cou et ils se placent ensuite à une extrémité dı champ arec un bàton pointu d'une main, en ligne droite à 2 pieds de distance l'un de l'autre. Ils suivent

(1) Annales agricoles de Roville, Ve livraison, p. $3{ }_{1} 6$. 


\section{$(: 25)$}

la ligne droite en faisant a chaque pats un tron dans la terre avec leur baton; ils y jettent quelques grains de maïs et les courrent de terre avec le pied. Arrivís à l'antre extrémité, ils recommencent dans un antre endroit.

Le procédé suivi dans quelques contrées de: l'Amérique espaynole est à peu près le mème, comme nous l'indique Francois de Neufelıàteau (1).

Les indiggenes choisissent de préférence, pour cette culture, les bords limonenx des rivieres des pays chauds, qui débordent chaque annće.

Ils font tremper le mais pendant douze heures, et sans autre préparation à la terre que de faucher et faire bruler l'herbe, ils sèment le maïs.

Un homme a un hàton de moyenne grosseur et de 7 à $S$ pieds de longueur, armé d'un fer an forme de coin, de trois travers de doigt de largeur sur 5 lignes d'épaisseur el 6 de hauteur. Il fiche ce bàton cu terre et l'ébranle une ou deux fois pour agrandir le trou; a chaque pas qu’il fait il en ourre $n$ autre,

(1) Onvage citi, P. 158. 


\section{(124)}

de manière à laisser 2 pieds de distance entre chaque trou; un petit garcon le suit immédiatement, il met quatre grains dans chaque trou et le recouvre de terre immédiatement.

Un homme peut ainsi, dans sa journée, ensemencer commodément un terrain de 200 pas ordinaires de longuenr sur 150 de largeur.

$6^{\circ}$. Avec le semoir à brouette.

Voyez, $\mathrm{Pl}$. I $/ 4$ et I 6 , dans la Description des instrumens d'agriculture, par Thaër. Paris, 1821 , in- $4^{\circ}$. Dans cet instrument, la lanterne qui sert pour semer les féveroles et les haricots sert aussi pour le maïs.

Ce semoir, dont parle M. Mathieu de Dombasle et qu'il emploie à Roville, est, je crois, à peu de chose près le même que celui qui est représenté dans le Londons encyclopredia of agricultura, p. 37 1.

$7^{\circ}$. Machine à semer, de J. Burger.

Les lecteurs qui voudraient des détails sur cette machine entrouveront la description à 
la page .52 de l'omrage de Burger, et lat figure dans les planches qui le terminent ( $\mathrm{r}$ ).

Cette machine a quetque ressembiance aree le semoir à hrouente; de est portie sur deux rones, le fer d'essieu de l'une est rond et l'antre earré; la rone dont l'essien est rond tourne tonjours, l'antre, an eontraire, he ronle qua volonté, c'est à dire, Jorsqu'on la pose í terre : cet effet se produit facilement en appuyant un peu plus avee la main droite ou avec la main yanche, suivant l'effet qu'on veut produire.

Voici son mode d'aclion:

Pendant que les roues de la machine tournent, le fer d'essieu à moitié carré, quii se trouve daus le trou de la roue gauche, lequel est igalement carré, et le rouleau se touruent éggalenent. Ce dernier recoit la semence d'en haut, et en tournant il la vide dans l'entonnoir par lequel il tombe trés prés derrière le couteau qui ourre la terre.

Si l'on ne fait aller la machine qu'avec la roue droite, alors le rouleau ne tourne pas,

(1) Ouvrage cité (en allematu). 


\section{( 126 )}

parce que le côté arrondi du fer de l'essieu tourne dans l'ouverture ronde de la rone. On attelle un cheval qui marche à pas éģal devant la machine. Si on suppose que l'on commence du côté gauche, on met la roue droite près d'une corde que l'on aura tendue pour aligner. Le conducteur du cheval le saisit par la bride du côté gauche et près de la bouche, et le conducteur de la charrue prend les deux poignées dans ses mains, soulève la charrue autant qu'il est nécessaire pour que le couteau s'enfonce dans la terre; il fixe continuellement la roue gauche, afin qu'en marchant elle ne s'écarte pas de la corde.

La première ligne achevée, le conducteur change de côté, prend le cheval à droite et le tourne, tandis que le semeur tourne la machine avec la roue droite par une version très simple, en levant un peu la roue gauche et en faisant de la roue droite le centre de gravité de a machine entière. La roue droite suit la même ligne qu'elle avait tracée en allant. Le charretier marche dans le sillon fait par le couteau it ou se trouve la semence, parce que ce n'est pas seulement la distance la plus convenable 


\section{(127)}

du cheral, mais parce qu il recoure ansi le grain de terre. Il doit dans cette ligne aller it droite, car s"il marchait à gauche, ses pas rendraient méconnaissable l'ornière de la rone ganche qui tourne derrière lui et qui indique le troisieme rang.

Il n'y a pas besoin d'un appareil pour convrir la semence; car l'entomnoir se trouvant tris près derrière le conteau, les grains tombent dans la terre an moment oì elle est ourerte par le fer : elle se ferme d'elle-mème aussitòt que le couteau avance, et cela si exactement, qu'on n'aperẹoit jamais un grain su' la surface.

En quatre heures on laboure et ensemence ainsi un arpent qui vant 300 pieds.

Ces différentes manières de semer ne sont pas les seules employées; car, sans mentiomer les procédés qui rentrent dans cenx dijà indiqués, nous voyons quelques peuples en adopter d'utiles à leur climat. "Ainsi, au "Sénégal, le grain le plus commun est une " espéce de millet, que les Indiens appellent " mais. On le sème sur la terre pendant qu'elte " 'st sèche, et on le couvre d'un pen de sable, 


\section{(128)}

" parce qu'on craint que, si on jetat de la terres " par dessus, la pluie et l'eau du fleuve Séné" gal débordé, venant à l'amollir, et le soleil „) à le dessécher extrêmement, ces deux excès " d'humidité et de sécheresse n'empêchassent. " le millet de croître ( $\mathbf{I})$. "

Harasti di Buda a fait une expérience qui lui a réussi à souhait. "Lorsqu'on fait, dit" il (2), la plantation ou l'ensemencement dı " maïs, il arrive quelquiefois qu'une partie du " grain ne germe pas; pour y remédier, il faut. " semer, dans un petit coin de terre séparé, de "bonnes semences de maïs, formant une es" pèce de pépinière, pour être mis plus tard à " la place des grains qui n'ont pas levé : ces " grains transplantés réussissent mieux que " ceux qui ont été semés. "

J'ai répété avec succès cette expérience, et quarante pieds, repiqués lorsquils avaient. dejà 8 pouces de hauteur, ont hien repris et.

(i) Description de l'Afrique, ouvrage traduit dn flamand d'O. d'Apper, D.-M., p. 231. Amsterdam, I 686.

(2.) Ouvrage cité (en italien), p. 58. 


\section{$(129)$}

donne d'anssi beamx épis que crox qui avaient éti semés sur place.

Suivant Andrienx, il fant 2 boisseaux de. maïs pour ensemencer mu arpent metrique.

\section{Culture intremédinire.}

Quoique quelques agrientem's pensent qu'il est plus profitable de cultiver le maiss seul, cependant il est asse\% d'usage de semer dans les lignes des plantes, qui augmentent ainsi le produit dn terrain.

En Alsace, on sème ordinairement des haricots blanes entre les lignes; on les sème, comme le maïs, par groupes de 5 ou 6 grains, de manière qu'une touffe de haricots soit entre quatre tiges de maïs, et réciproquement. Dans quelques endroits, on procède avee moins de sỵmétrie, parconséquent moins bien : on sème les haricots ì la volée et on les enterre en plantant le maïs. C'est le haricot nain dont il est ici question; il n'atteint guère qu'un pied de hauteur et n'a pas besoin d'être ramé. Je ne conseillerais pas, dit M. Schwer\%, de planter la grande espèce qui monte, à la vérité, après 


\section{(150)}

le maïs, mais qui par cela même empèche lo maïs de muirir daus une année tant soit peu humide. Quand le champ est ainsi planté de maïs et de haricots, on sème encore à la volée. environ 6 litres de graines de chanvre par hectare : d'autres sèment et enterrent ce dernier en même temps que les haricots et de la manière que nous avons décrite plus haut. On sait que le chanvre ne peut faire de bien au mais, mais n'importe, pourvu que le cultivateur y trouve son bénéfice ( 1 ). Il est hors de doute qu'une triple récolte en maïs, haricots et chanvre pour semence, ce dernier, quoiqu'en petite quantité, doit fortement épuiser le sol; mais le tas de fumier est là qui remédie à tout. A Sultz, le champ de maïs est bien autrement mis à profit; on y plante, entre les haricots, des choux blancs, des chouxraves et autres légumes. La terre que l'on des-

(1) Le but de cette pratique est d'obtenir une graine de chanvre qui est beaucoup préférable pour la semence à celle que l'on récolte dans les chenevières, parce que les plantes qui la portent reçoivent mieux les influences de l'air. (M. Dombasle.). 
line au maïs est le jardin de l'annére, et l'on n'épargne ni travail ni fumier pour le mettre (nil état : elle est cultivéc aver le plus grand $\operatorname{sonin}(1)$.

En Bourgogrne, on séme, surtout dans lo petit Turquie, des haricots nains qui y viennent fort lien.

Une bonne méthode consiste, apres le troisieme binage, i semer entre les lignes des raves, on bien planter des choux pour la cuisine et la nourriture des bestianx.

Quelques agriculteurs alternent avec avantage les lignes de maïs avec des lignes de ponmes de terre, plusieurs espèces de haricots nains, de millets, de betteraves ordinaires, de navets, de carottes, de citrouilles.

Ils prétendent, pour les ponmes de terre, que le maïs, empéchant la croissance des tiges, favorise la multiplication de ses tubercules.

En Espagne, on y seme sourent de la spargotlte.

(1) Ouvage cité de: Schwerz, p. 315. 


\section{(15\%)}

Les Iroquois y mettent l'helianthus tuberosus, ou topinambour.

En Anérique, on préfère y mettre des graines de navets ou féveroles.

A la Nouvelle-Orléans, ce sont des melons d'ean et des giraumons.

Parmentier ( I ) parle d'un usage qui existe aux Antilles de planter le maïs eutre les plants de cannes à sucre et de café. Un de mes amis, et confrère, M. le docteur Hibon, né à l'IleBourbon, et qui est propriétaire d'une caféterie considérable, m'a assuré que cette méthode y était totalement abandonnée, parce que l'on avait reconnu qu'il nuisait à la récolte du café.

Quelques auteurs, parmi lesquels on peut citer Lelieur et Gothard, pensent qu'on ne doit jamais rien cultiver entre les lignes du maïs. Ce blé, dit le premier, pour bien croìtre, a surtout besoin d'air : ainsi, dans un champ de mais, les bonnes plantes ne doivent pas être plus souffertes que les mauvaises herbes.

De tous ces avis contradictoires, il fant conclure que cette culture doit devenir un

(I) Ouvrage cité, p. $9^{3}$. 


\section{$(155)$}

point d'étude pour chaque cultivateur : c'est ¿ lui d’apprécier la bonté du sol qu il destine an mais, son exposition plus on moins favorable, et le temps qui lui est nécessaire pour que son grain arrive i une parfaite maturite.

\section{Utilite du mans dans les jardins.}

Un peut annuellement faire servir le maïs de haie pendant le temps de certaines récoltes; pour les jardiniers, surtout pendant la saison des fruits et des légunes. Cette plante se séme en avril; plle flemrit en juillet, en s'élevant à 5 ou 6 pieds de hauteur; elle fructifie en aoùt. On recueille l'épi au mois de septembre ou d'octobre. On peut, si l'on reut, laisser subsister en haie sa canne, et la renouveler aimsi tous les ans. Il est des jardiniers qui n'entourent pas autrement leurs rerger's et potagers, et (qui soutiennent les masses de maïs entr'elles en les liant par leurs longues fenilles ou arec de l'osier (1).

(1) Amoreux, Mémoure sur les haie's, couronué par l'Académie des sciences, belles-leteres el arts de I,yon

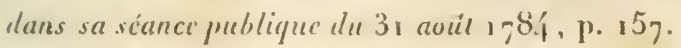




\section{$(134)$}

Le maïs est propre à former des abris soít contre le chaud, soit contre le froid, dans les jardins, autour desquels il se plante sur deux langs, à 6 pouces de distance; vide contre plein ( I). Il sert de bordure aux divers légumes, et comme il s'élève très haut, il permet à plusieurs plantes herbacées et à plusieurs racines de croitre et de mùrir à ses pieds avant qu'il ait atteint lui-même sa maturité. On peut donc semer, auprès du mais, de petites salades, du cerfeuil, du persil, des raves et radis, des citrouilles, ete.

\section{Culture ordinaire.}

Je donnerai ici surtout la description des nouveaux procédés qui sont très bien décrits par Schwerz (2), parce que dans Parmentier on ne trouve que les moyens de culture usitís à l'époque où il écrivait.

Le maïs a besoin des mêmes cultures que

(1) Nouveau Cours complet d'agriculture, I. IX, p. 2.75 .

(2.) Schwer\%, ouvragge cité, p. 317 et suiv 
le tabar. Anssitòt qu'il est ì 7 ou 8 pouces hors de terre, on lui donne le premier binage (1), et quand il a atleint. 10 ponces, il reçoit cetle culture pour la densieme fois. On commence ators dejal a le buter un tant soit peu, et on arrache les plantes superllnes; car il est à remarquer qu'on n'en laisse qu'me, el quand les distances sont grandes on en laisse au plus deux. Quand il a atteint un pied et demi, on le bute pour la dernicre fois. Il fau faire bien attention de ne pas domner ces cultures par un temps humide (2).

Le binage est indispensable dans la culure du mais. On ne peut jeter trop de terre contre les tiges de cette plante, autant pour faire pousser les racines an collet que pour la gat-

(1) Près d'Alby, le travail se fait arec la lione plate, nommce foussou en patois.

(2) M. de Beammont conseille de ne donner la troisicuse laçon qu'après la suppression de la partic supirieure des tiges, dont nous allons parler, attendu que cette opération pourait délruire, au mojen du piélinenent, l'ellet du labour antérieur, tandis qu'en faisaut le labour après la récolte dis fourrage, la serre reste meuhle jusqu'au moment de la ricolte des epis 


\section{$(156)$}

rantir de l'humidité et des coups de vent (1).

On fait souvent cultiver le maïs à la tàche. On paie $4 \mathrm{fr}$. par 20 ares dans toutes les terres sablonneuses, comme à Hoerdt, ce qui fait $80 \mathrm{fr}$. pour les quatre cultures d'un hectare. Ces frais sont considérables ; mais il serait injuste de les mettre à la charge du maïs, puisque l'amélioration qui en résulte agira sur plusieurs récoltes subséquentes. Dans une exploitation un peu considérable, ces dépenses peuvent être réduites de beaucoup, quand on peut faire exécuter ces cultures avec la houe à cheval et avec le butoir; elles n'en seront peut-être que mieux faites.

A cet effet, on serait obligé d'introduire un léger changement dans la manière de planter, et il faudrait se procurer ces deux instrumens. Dans une exploitation où l'on ne cultive qu'un

(1) Chaque butage fait pousser un rang de racines au dessus des premières, de manière que, par le nombre des couronnes, on peut juger du nombre des butages.

- Les buttes doivent ètre un peu aplaties, et même un peu excavées autour du pied de la tige, afin de donner attx eaux pluviales le moyen d'abreuver toutes les racines. 


\section{$(157)$}

hectare de maïs, le prix de ces instrumens serait payé an bout de deux ans pár l'économire de main-d'euvre, et ceci est d'autant plus rai que ces instrumenș sont, du reste, trés propres à la culture de la pomme de terre ret du colyil.

Lorsque les binages ont lien ì main d'homme, on n'en donne qu'un on denx an plus, et ces operations se font généralement en juin ou juillet. Elles occasionent une dépense de 20 it $25 \mathrm{fr}$. par hectare pour linage, attendu que douze journées d'ouvriers, à $2 \mathrm{fr}$., y sont nécessaires. De plus, ces binages sont loneys et s'exécutent à des éproques où souvent l'aggriculure réclame le concours indispensible d'un grand uombre d'our riers.

Avec la houe à cheral (1), le maïs recoit trois binages, deux en juin, um en juillet; une demi-journe d'un cheval et de son conducteur suflit pour ce travail, et la dipense, pour chaque opération, ne peut ètre de plus

(1) Description des instrumens les plus utiles on agriculturc, par Thaer, fir. $146,1 / 7,1.18$. Paris, 1821, in- $4^{\circ}$. 


\section{$(158)$}

de $7 \mathrm{fr}$. par hectare, ce qui occasione une dépense totale de $2 x$ fr. au plus, landis qu'en employant la binette elle s'éleverait à $50 \mathrm{fr}$. ( I ).

La manière de planter le maïs à cet effet est celle-ci. On met les lignes à 3 pieds de distance, comme à l'ordinaire, avec la différence cependant que dans les lignes les grains seront posés isolément à un demi-pied l'un de l'autre. Comme il n'est pas nécessaire de procéder avec une grande exactitude, on peut, au lieu de déposer péniblement la semence en terre, la jeter avec un tant soit peu de précaution dans la raie. Si l'on a un semoir sous la main, il est préférable à tout. Aı lieu de la houe à main, on se sert de la houe à cheval pour biner les intervalles des lignnes (2).

(1) Notice de M. Debonnaire de Gif dans l'Instruction de $\mathbf{1} 830$.

(2) Lorsque l'on bine entre des plantes un peu élevées , comme du maïs, etc., on supprime le palonnier que l'on fixe ordinairement au crochet d'attelage, et au lieu d'atteler les traits aux extrémités de ce palonnier, on les réunit et on les fixe au crochet lui-même; mais afin d'éviter que les traits ainsi placés frottent les cuisses du cheral, on les ticnt écartr's à une dis- 
Quand les plintes ont atteint 8 a 10 pouces, on arrache les pieds superflus, de maniene quil ! ait 24 pouces de distance d'une plante à l'autre (1).

lauce suflisante, au uogen d'un baiton que l'on place lorizontalenent cntre'ux, immóliatement derrière les jarrets de l'animal: ce baton, placé ansi, se troure assez éleve ponr ne pas nuire atux plantes, conne le lerait le palomnier, qui pourrait en casser ou coucher quelques unes. Annales agricoles, Ve livaison, p. 387.

(1) Je ne comseillerai jamais de netre les lignes ¿ 2 pieds et les plantes à 15 ou 18 pouces en ligne, comme cela est décrit daus les Principes de l'agriculure raisonnée, $\$ \mathbf{I V}$, p. 333; car, outre qu’il serait très difficile de cultiver le maïs a des distances anssi rapprochées, il ne murirait pas dans des amnées humides, el ípuiserait trop le sol, comme on en a fait l'expérience en Italie. De cette manière, on manquerait une partie de ce que l'on se propose en cultivant le mais, en ce qu'il ne serait plus une récolte préparatoire pour le blé, et le proverle qui dit que les semailles épaisses produisent de petites récoltes se trouverait vérifić. On pourrait cependant, pour se procurer une plus grande quantité de fourrages, mais (n maintenant toujours l'intervalle de 3 pieds entre les lignes, laisser mouter jusqu'à un pied et demi les liges avant de les arracher. (Nole de M. Schwerz.) 


\section{$(140)$}

Le butage s'exécute en deux ou trois fois mieux et plus régulièrement avec la charrue et le butoir qu'à la main. De cette manière, le maïs se trouve sur de longues crêtes qui vont d'une extrémité du champà l'autre. Je ne recommande cette méthode que pour les pièces de terre longures et étroites, on de largeur ordinaire; celle que je vais décrire est préférable sur des pièces carrées ou de largeur convenable.

Quand le champ a été labouré et égalisé avec la herse, on tire avec une charrue sans versoir ou avec un versoir très étroit des raies très peu profondes, à 3 et même 4 pieds de distance l'une de l'autre : on croise celles-ci par d'autres tirées en travers et à la même distance. Aux points où ces raies se couperont, on plantera quatre ou cinq grains de maïs, sur lesquels on poussera un peu de terre avec le pied, pour les courrir légèrement. De cette manière, on peut parfaitement cultiver en long et en large, et chaque butte sera isolée, comme dans la culture à la main. On laissera tout an plus deux tiges par butte. On ne pourra y planter de haricots ni semer du 
chandre, attendu quivee la home it cheval on me peut les mentrere comme avere la home simple; mais le maïs sera d'un rapport plus considérable et phus sir. De plus, la terre recerra les preparations dont elle peut avoir besoin.

Voici comment M. Indrieu faisait cultiver son maïs, à Cheptainville, situé à 56 kilnmetres ( 9 lienes) sud de Paris (1).

Le terain ! est en partie léger et composi d'argile el de sable, en partie gravelemx, paree qu'il repose sur de la terre de menliere.

Le fumier des bergeries, enfoui an printemps, dit-il, au moment où la terre entre en sive, fermente et favorise la gemination du mais, je lai prefieri. On donne, lorsqu’il est possible, un premier lahour avant l'hiver et In second à la fin de mars.

On plante dn 10 an 20 avril; il est désirable qu'on ne passe pas les premiers jours de mai.

Deux boisseanx suffisent pour ensemencer un arpent métrique; les épis ne sont égrenes

(1) Mémoires de la Socièté d'agriculture de Scinsel-(Jise, 1. VI, p. 53 el suiv. 


\section{(142)}

qu'au moment, on n'en prend que le pied pour avoir des grains plus gros et plus sains; avec ce procédé, je n'ai jamais éprouvé aucune des maladies que M. Parmentier a olservées.

Avant de planter, la terre n'est point hersée, mais seulement roulée. Des enfans placent les grains, au nombre de trois ou quatre, isolément, en laissant toujours une raie vide entre deux, en sorte que la distance soit d'environ 18 pouces en tous sens.

Lorsque le temps est froid, le maïs reste long-temps en terre, le champ se courre d'herbes; on le nettoie avec la herse, le maïs qui commence à pointer ne peut en souffrir aucun dommage. On lui donne une première façon lorsqu'il a 6 à 7 pouces : c'est alors que, si dans une mème fosse plusieurs tiges se tonchent, on supprime celles qui sont très rapprochées, et dont les racines, qui sont très étendues, se nuiraient réciproquement.

L'opération de buter est le complément de ce genre de culture; cette façon, donnée avec soin et au moment convenable, m'a le plus ordinairement procuré des épis bien nourris, 


\section{(1.13)}

el dont les grains sont aussi formés jusqüi lenr extrémitr que dans les départemens miridionalux.

Vers le 15 juilled, on rommence à roir les flemrs ot les styles. Ces épis naissans, au nombre de deux ou trois an moins sur la même tige, ont hientôt 5 ponces de longueur; il ne faut bientót plus qu'un peu de beau temps pour obtenir une récolte superbe.

M. Picot de la Perrouse indique, dans te volume $L^{\mathrm{e}}$ des Amuale's de l'agriculture française, la manière de culliver les terres pour le mais daus le Lauraguais (petite contrie du Haut-Languedoc).

A l'lle-Bourbon, arant la plantation du maïs, on fait remuer la terre par les noirs avec la gratte, instrument assez semblable à notre houe, mais qui porte un manche heancoup plus long. Il se plante en décembre, il serait dejà trop tard en janvier : pour cela on fait des trous à la distance de 2 pieds, on met quatre ou cinq yrains dans chaque; il sort de terre au bout de quatre ou six jours.

Los'squil a atteint 6 à 8 pouces, on $1 c$ degarnit, c'est à dire qu'on ne laisse qur 


\section{$(1 / 4)$}

trois pieds par chaque touffe; il faut douze it quinze jours pour qu'il arrive à cette grandeur.

On débarrasse le maïs des herbes qui l'entourent en faisant gratter la terre. En juin, les épis sont bons à être cueillis et la récolte se fait.

On plante entre les pieds des citronilles, des melons et deux espèces de pois (haricots) appelés pois du Cap et pois de Bembelok, qui sont tous bons à manger en vert; très propres aussi étant secs à la nourriture des animaux, et ayant encore l'inestimable avantage de bien couvirir la terre, de la préserver du soleil, d'y entretenir de la fraîcheur et de beaucoup l'améliorer.

Cette double récolte n'empêche pas la plante d'atteindre jusqu'à 8 pieds de hauteur dans les bonnes terres; cependant, près du rivage de la mer, du côté de Saint-Leu, il est beaucoup plus petit.

Je renvoie ceux qui voudraient des détails sur cette culture à Bourbon, au Mémoire de M. Villèle, inséré dans les Annales de l'agriculture firançaise, Paris, an XI, tome XIV, p. 170. 


\section{$(1 / 45)$ \\ Elagrage et citêtement du maïs.}

Le maïs pousse ordinairement près de terre des tiges latérales, qui ue produisent que de petits épis, ou qui, le plus souvent, sont stiriles; si elles n'enlevent pas une partie des forees de la tige principale, elles contribuent du moins à épuiser plus fortement le sol. On a soin de les arracher, elles fournissent un fourrage précienx.

Les petits épis, placés ordinairement au dessousdes autres, doirent être aussi enlevés. Celui qui ne se pressera pas de faire cette opération ne retirera du maïs que des épis vides, petits et peu garnis de grains, car ces faux épis sont alors ceux qui se nourrissent du sue qui aurait dù alimenter les épis qui donnent le fruit. De scmblablesépis doivent ètre regardéscomme des plantes parasites qui privent la plante de tout son suc nourricier. En même temps qu'on séparera ces épis du pied, on ôtera encore quelques feuilles inférienres de la plante qui sont voisines de terre; elles sont inutiles, et en se séchant elles nuisent par leur quantité 


\section{( 146$)$}

à la végétation. On ne laisse qu'un ou denx épis à la tige principale, et les autres sont enlevés en même temps que les pousses dont je viens de parler.

Dans les mois de juin et juillet, le maïs se trouve dans la plus parfaite vigueur et à sa plus grande hauteur. Les fleurs mâles commencent à paraitre; elles contiennent une poudı 'e très fine, laquelle sert à féconder les fleurs femelles.

C'est à cette époque que la fécondation commence. Durant ce temps, à moins que la nécessité ne l'exige, on derra ne pas entrer dans le champ, pour ne pas faire tomber par le froissement la poussière que contient la fleur mâle, car elle se trouverait perdue avant le temps de la fructification. Si cependant il était nécessaire d'y entrer, on devrait le faire avec beaucoup de précautions; car, sans cela, le cultivateur ne recueillerait que des épis grêles et peu garnis de grains. On doit, à plus forte raison, prendre garde de couper ces fleurs mâles lorsqu'elles peuvent encore servir à l'importante fonction de la fécondation. Quand la fleur mâle dı mais commence à se 


\section{$(1 / 7)$}

faner, c'est un signe que lit fécondalion est termince. Cette fleur ou aigrette n'est plus alor's nécessaire, el l'on coupe la tigre prés de la fenille qui se trouve au dessus de l'épi le plus élevé. C'est ce que l'on appelle ététer le maïs. Les agrieulteurs alsaciens ne sont pas d'aceord sur l'utilité de cette opélation. Les uns prétendent qu'il raudrait mienx laisser lit tige en. tiere; les autres, an contraire, disent que l'on accélère ainsi la maturité : c'est ponrquoi ils pratiquent l'étètement, particulièrement dans les années froides et lumides. Il n'est pas sạns vraisemblance que la croissance ultérienre de la plante soit arrètée par suite de la blessure qu'elle vient de recevoir, que l'astiviti dans la circulation des sucs cesse, el que, faute du renourellement de ces sucs, le fruit sèche plus tòt. Il en résulterait une diminution dans le volume des grains, mais une récolte moindre en bon état vaudrait toujours micux qu'une plus considérable que l'on ne pourrait pas rentrer dans un état satisfaisant. Dans des climats plus chauds, cela pourrait être blàmable; mais en Alsace, où le maïs ne parvient pas toujours à sa maturité, je regarde celle pratique comme 
très louable. Dans tous les cas, la diminution dans la récolte des grrains est bien compensée par la quantité des sommités données vertes aux bestiaux. Quelques agronomes conseillent en outre d'ôter la plus grande partie des feuilles qui embrassent les épis fruitiers, laissant seulement la quantité de feuilles nécessaire pour les envelopper. Ce dépouillement des feuilles superflues qui enveloppent les épis fruitiers se fera pour que l'air et le soleil puissent les atteindre et leur permettent d'arriver à maturité.

\section{Culture comparée.}

M. Andrieu de Cheptainville a fait la comparaison de l'orge et des haricots avec le maïs. Je vais le laisser parler ( 1 ):

" J'ai pensé que la comparaison de deux productions auxquelles j'ai substitué celle du

(1) Mémoires de la Société d'agriculture du département de Seine-et-Oise, t. VI. Mémoire sur la culture et les produits du maïs, par Andrieu, p. 53 et suiv. 
maïs (I'orge et les haricots), présentée aver les dépenses et les risultats, pourrait faire ourir les yeux des cultivateurs du département de Seine-et-Oise sur des bénéfices donı je fais la démonstration. Il sera facile de connaitre, par le tableau ci-joint, que, bien loin de diminuer le montant des avances sur l'un ou l'autre article, j'en ai préférél'exagération. J'ai indiqué, par exemple, des labours an prix de .24 francs par arpent métrique, et cependant, dans des anuées comme celle-ci ( 8 । 5), les gelées, qui s'étitient manifestées vers la fin dumois de novembre précédent, n'avant presque pas en d'interruption, un seul labour a sufli. Je me suis également gardé d'élever les produits, un taux moyen a suffi. L'orge dome quelquefois, surtout en petite culume, entre 24 et 36 sacs d'un hectolitre el demi pour un arpent métrique.

"Il est rai que le mais demande plus de culture que l'orge, mais aussi il nettoie beaucoup micux la terre, et l'on est soment obligé de remédier au mal qu'a produit l'orge sous ce rapport. Au reste, il serait injuste de mettre sur le compte du mais tous les frais de culture 


$$
\text { ( } 150)
$$

qui doivent agir pendant plusieurs amnées et sur plusieurs récoltes.

"Les haricots peuvent produire entre quinze et vingt sacs, comme en $18 \mathrm{Ir}$; mais alors la saison qui leur est favorable l'est également au maïs : aussi, en la même année 18 I I, la production bien constatée du maïs a été de cent sàcs sur 2 arpens métriques.

„Ce dernier a un avantage extrêmement précieux. J'ai l'expérience que l'orage désastreux du 13 juillet 1788 , qui avait ravagé le territoire de ma commune dans toutes ses productions, et notamment les haricots, avait été impuissant sur le maïs.

"J'ai eu occasion de faire voir à des membres de la Société qu'un champ ensemencé en blé, après une récolte moyenne de maïs, soutenait la comparaison, pour la force et la beauté des épis, avec un autre qui avait été ensemencé en vesce et en haricots, qui est la manière la plus ordinaire d'alterner dans mon pays." 


\section{TABLEAU WE COMPARASON}

Des arunces et des produits d'un arpent metrique de terre labourable e't orge, haricots et maïs.

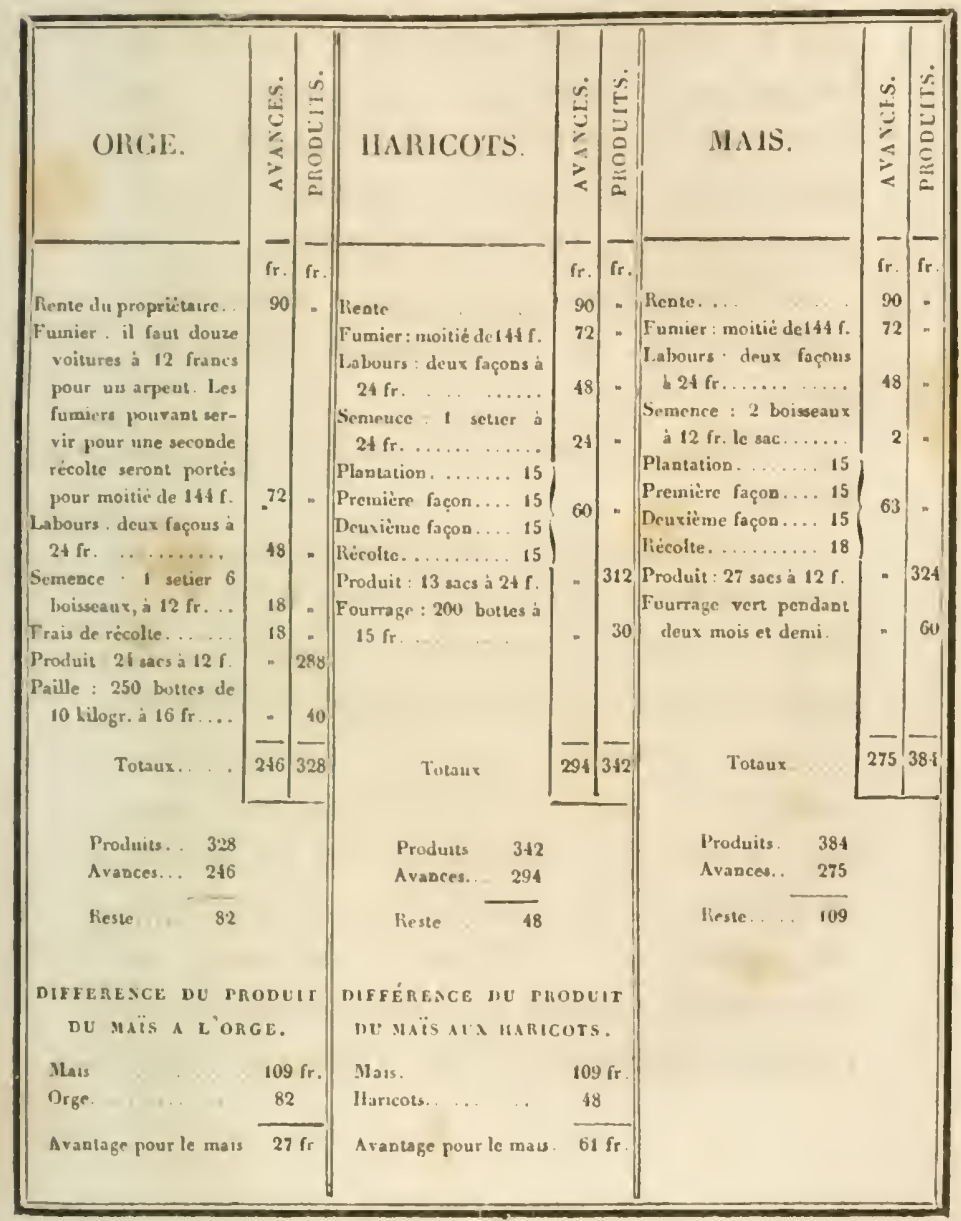




\section{Récolte.}

Le mais donne une récolte plus sûre que celle du froment. Le temps de la récolte est indiqué par la dessiccation de la plante, par la couleur de l'enveloppe des épis, dont les feuilles sont alors jaunes et sèches, et aussi par la couleur et la dureté du grain.

En Bourgogne, pour le gros maïs, c'est ordinairement du $\mathrm{I}^{\mathrm{er}}$. septembre au $15 \mathrm{oc}-$ tobre.

En Italie, la récolte commence au mois d'août.

La récolte du maïs, en Alsace, a lieu vers la Saint-Michel, et quelquefois ver's la fin d'octobre. Quand le mais est mûr, l'extrémité des feuilles quientourentl'épi blanchit, s'entr'ouvre et laisse apercevoir ce dernier. Cependant, si le froid venait à se déclarer avant cette époque, il faudrait le rentrer tel quel. Les feuilles se fanent, pâlissent, les grraines se dessèchent, tout espoir de le voir mûrir davantage est alors perdu. Comme les gelées nocturnes se font plus fréquemment sentir dans les situa- 


\section{$(1,5)$}

tions basses quailleurs, il ne serait pas prudent d'y planter elıe nous le maiss. Sans cette circonstance, les marais et les étangrs desséchés où le blé a coutume de verser seraient très propres à la culume de cette plante.

On coupe les tiges près de terre avec la faucille, on les transporte de suite à la maison, où on les dépouille aussitòt de leurs épis : celles-là sont alors exposées en plein air, pour ètre séchées. Quelquescultivateurs, cependant, cueillent les épis pendant que les tiges sont sur pied, arrachent ensuite celles-ci, les posent en faisceaux et les laissent ainsi sécher jusquien hiver (1).

- M. Andrien (2) donne ce conseil, il indique aussi de les mettre en tas sur le chan!), d'espace en espace; lì, ils achèvent de mùrir, les enveloppes se séparent plus facilement et les grains se détachent mieux : ils peuvent rester ainsi tune semaine sans inconvénient. Onl les transporte ensuite dans une cour en pleiı air;

(1) Annales de Roville, Ve livaison, 1. $322 \mathrm{ct} 323$.

(2) Mémoires de la Sucicic d'agrionluure de Scineet-Oise, t. VI, p. 6. 


\section{( 154 )}

en masse, sous un bâtiment, ils s'échaufferaient.

Quelques personnes pensent qu'il faut éviter de laisser les tiges à la pluie après la récolte des épis; elles contiennent encore beaucoup de substances sucrées que les eaux finissent par entrainer avec elles. L'usage où l'on est de laisser, dans certains cantons, les tiges en meules debout dans les champs et dans les cours doit donc être réformé.

\section{Rapport.}

Amoreux, dont le mémoire concourut, avec celui de Parmentier, pour le prix de l'Académic de Bordeaux, en 1784, dit, dans une lettre ì Mrançois de Neufchâteau (I) : Si j'avais une propriété rurale facilement arrosable, je voudrais, avec le seul maïs, en faire le sol le plus productif, un jardin égal à celui de Montezuma par la richesse (voir page 19), à l'exception qu'il n'y aurait pas de gerbes d'or.

(1) Ouvage cité, p. 115. 


\section{$(155)$}

Le maïs est, sans contredit, la plante yui foumit le plus de grains.

Lorsplu'on sime le maïs en plain champ comme le blé, il est maigre, chétif et ne rapporte qu'un épi; mais si l'on sème les grains i 18 ponces an moins de distance dans les lignes, il rapporte souvent plusieurs grappes.

Un hectare cultivé en maïs peut contenir plus de quatorze mille tiges lorsqu'elles sont à une distance d'environ 75 centimètres, en tous sens. Chaque tige contient an moins un épi et presque toujours deux; en n'évaluant quw trois épis pour deux tigges, il y aura une récolte de vingt et un mille épis.

D'après des expériences, il parait démontré que einc épisde gros maïs contiennent approximativement plus de gyrains qu'il n'est nécessaire pour remplir un litre.

Cinc conts épis procureraient donc un hectolitre de grains el par suite les vingt et un mille épis que renferme l'hectare assureraient 111 produit de 42 hectolitres de sirains.

En retranchant 2 hectolitres pour les épis front les sommites seratent vides, pour les 
grains avariés, il y aura en somne un produit de 40 hectolitres de grains.

La valeur de l'hectolitre de maïs doit être estimée approximativement à 20 francs; mais en ne la calculant que sur une base de 15 franes, les 40 hectolitres donneront une somme de 600 francs; ce qui constitue le produit brut en grain seulement d'un hectare cultivé èn maïs.

Les dépenses dont on donne le détail, p. 26 de l'Instruction de 1830 , se montent à 320 francs; le produit net est done de 280 ( 1 ).

En Bourgogne, dans 34 ares de terrain on peut récolter de 20 à 40 doubles décalitres suivant la force du terrain, e'est à dire en terres sablonneuses ou en terres fortes.

Le petit Turquie de Romanie étant sec peut y peser 18 à r 9 kilogrammes le double boisseau lorsqu'il est en grain, il diminue d'un kilogramme et demi à peu près en le réduisant en farine.

Je lis, page 209, de l'ourrage de François

(1) Instruction de 1830 , notice de M. Debonnaire de Gir. 
de Neufehâtean, ce qui snit (1): Il a résulté, d'une expérience que jai soment répetere qu'un sac de blé, de la mesure que l'on employait en $179^{8}$ dans le département de la Haute-Garome, pése r 80 livres, quil fournit 145 liveres de farine at 34 livres de son; et qu'un sate de maïs de la plus belle qualiti pese 170 livres, foumit 153 livres de farine et 16 livres de son; il y a une livere de déchet.

En Alsace, suivant M. Schwerz, le produit du maïs est de deux à huit saes, 2 hectolitres 34 litres a 9 hectolitres 56 litres par arpent de 20 ares. Le produit moyen serait done de 29 hectolitres par hectare, le produit d'un arpent de maïs est à son produit en orge comme 7 est à 5 ; on, ce qui revient an mème, 5 arpens de maïs produisent autant que 7 arpens d'orge, et le fourrage que produit le mais vaut bien la paille d'orge.

En Toscane, un champ planté de maïs rapporte, année commune, de deux cent quarante à trois cent soixante fois ce que l'on a semé.

Dans les arrondissemens de Cera et d'Acqui,

(1) Franç. de Neufchâteau, ouvrage cité, p. 209. 
le blé rend quelquefois dix pour un, semence déduite; en Ligurie, jusqu’à sept pour un; et dans les pays montagneux, où le terrain est léger et sujet à la sécher'esse, le rapport est entre cinq et trois. Le maïs rapporte partout jusqu'à trente pour un, quelquefois jusqu'à cent. Dans les plus mauvais terrains il rapporte encore dix à douze, lors des bonnes années.

Dans les meilleures terres de l'arrondissement d'Acqui, dans celles qui se prêtent plus à l'irrigation, le maïs rend cent vingts pour un, dans les terres de deuxième classe quatre-vingts pour un, dans celles de troisième le tiers seulement de la grande production. Cette troisième classe étant la plus considérable, on ne peut calculer qu'à cinquante pour un au plus le produit moyen.

Il faut, dans le département, un-demi quintal de semence par arpent (1).

En Italie, si la saison a été favorable au maïs, la récolte est de 3 setiers au moins par arpent, sans compter les produits des autres

(I) Statistique du département de Montenotte, p. 200 et suiv. 
plantes qu'on sime presque toujours dans les espaces videslahourés, à dessein de détruire les mauraises herbes et de fortilier la tige.

Dapres Kalm, on a en Amérique me année de disette, lorsque le mais ne rend pas deux cents fois la semence.

Le maïs est généralement cultivé au Brésil, il fait la base rle la nourriture des blanes. II mûrit en quatre mois, et le produit est sou-vent de deux cents pour un; on assure rpu'il a dome quelquefois quatre cents pour un dans des terres de premicre qualité, par exemple dans celles dont on venait récemment de brùler les bois quil les courraient.

Du temps de Laët, qui écrivait il y a deux cents ans, on obtenait dejjà d’abondantes récoltes. Voici comme il s'exprime (1) : Mruisinm indicum quod I'Tirginicuses pagatow vocant hic uberrime nascitur (australis Virginice pars), et grana modo mbra, modo flava, modo mixti coloris et clegantissime

(I) Nowus orbis seu descriplionis India occidentalis, auctore Joanne de Laet, 1. VIII, p. 88. Lugd.-Bat., 1633 , in-fol. 


\section{(16o)}

variegata prodncit, in sex, septemve interdum, et decem pedum altitudinem assurgens et spicas tres quatuorve, nonnunquàm tamen et unam tantum, ferens, quingentis, sexcentis, et quìm uberrima messis est, seplingentis granis onustas singulari soli luxuriantis indicio. "

Feu Andrieu de Cheptainville, qui se livrait à la culture du maïs pour en faire des cornichons, dit qu'un arpent métrique peut fournir soixante millions d'épis propres à confire, lorsque la saison est favorable, et cela en moins de trois semaines.

\section{Commerce du maïs.}

D'après Arthur Young, en i 784 , on exporta de France pour 653, 100 livres de blé de Turquie ( 1 ).

La Hongrie, en 179\%, exportait par an So à 100, coo quintaux de maïs (2).

(I) Toyage en France, traduit de l'anglais, par F. S., 1. III, p. 27 I. $2^{\circ}$ édition. Paris, I 794 , in-8 ${ }^{\circ}$.

(2) François de Neufchâteau, ouvrage cité, p. I 76. Extrait d'un mémoire de feu $\boldsymbol{M}$. de Villeneuve. 


\section{$(16$,}

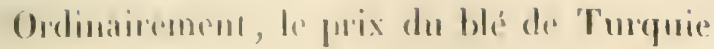

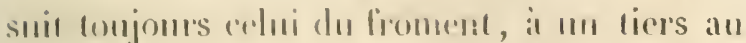
dessous, de facon yure si le setier de fromeut me vamt que i 5 l'anes, colui de 'Purquie en vall 10 .

J'ai chorche it me procures aupres du Ministre de l'Interien des renseignemens sur le enumeree actucl du maïs cu france; les registres des domanes confondent toujours dans le même article le maïs et le milled, le mais et le seiggle, le maïs et autres mentes graines, de facon qu'il est impossible d’indiquer exactement la quantité du maïs récolté en France. Je n'ai pu avoir que les renseignemens suivans, que lon trouvera sans doute fort imparfats.

En 1824 :

Importations pour les entrepôts. .

quitsux metriytu's

Importations versées directement à la cousommation, saus toucher aux cutrepôts. . . . . . . . 3

Exportations. . . . . . . 5,284

Réexprrtations. . . . . . . 44 


$$
\begin{aligned}
& (162) \\
& \text { Prix. }
\end{aligned}
$$

Le prix moyen d'un hectolitre de mais a iti, d'après les relevés faits sur les marchés:

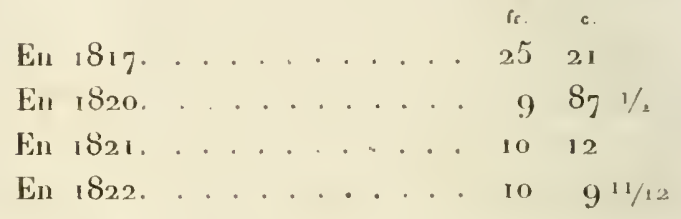

Quoique tous les registres m’aient été connmuniqués, je n’ai pu en tirer d'autres indicalions. 


\section{TABLEAU GENERAL}

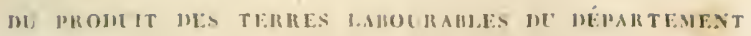
IBE YUVT: IUTTI. (1).

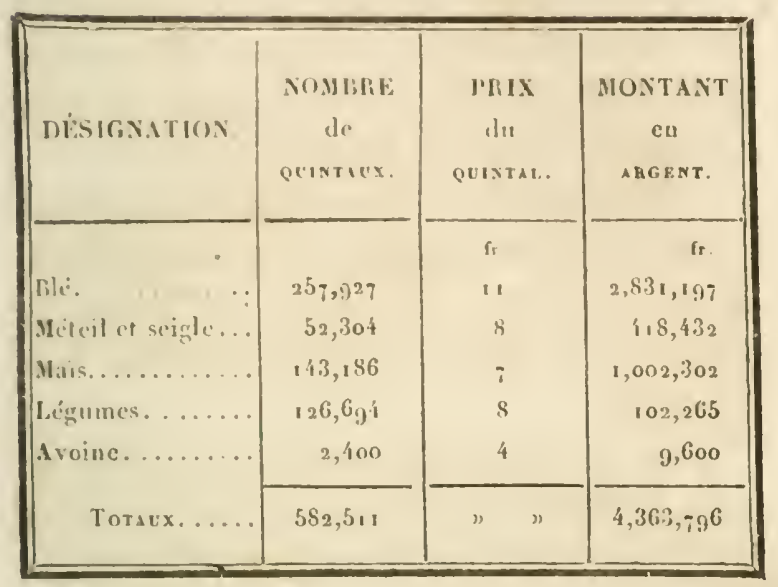

Les experts du pays estiment que la feuille du maîs, détachéc de sa tigge, est à peu près le sixicme du poids du produit; par conséquent, le prodıit du maïs ćtant de 145,186 quintaux, la feuille sera à peu prés de 23,864.

(1) Statistique du département de Montenotte, p. 203 et 204 . 


\section{( 164$)$}

Le prix est de 2 francs, le double de celui de la paille, le revenu est done de $47,728 \mathrm{fr}$.

Conservation du maïs en épis.

Le mais récolté est transporté dans un lieu see, vaste et couvert, où il faut, sans tarder, le dépouiller de sa robe. Voici comme Parmentier dit que l'on doit y procéder (I):

"Des femmes et des enfans s'asseient autour du tas, prennent l'épi de la main gauche, de la droite tirent les robes vers le bas, et eassent le noyau ou le pied aucuel elles sont attachées.

" On fait commumément, de la récolte du mais, trois lots : les épis les plus beaux, les plus mûrs sont mis de côté avec une partie de leurs enveloppes et destinés pour les semailles; les épis entièrement dépouillés de leurs robes, pour les usages ordinaires; enfin, les épis moins mûrs, et auxquels il semble que la végétation n'a pas été favorable, sont séparẻs du tas, pour être dépiqués par jour et servir de nourriture aux cochons et à la volaille."

(1) Ouvrage cité, p. I41. 
Dessiccation du mais par l'intermède de l'air.

Quoiqu'on puisse conserver le maïs autrement que par le secours de l'air, e'est surtout au moyen de cet agrent qu'il peut se perfectionner et conserver au moins quelques qualités on propriétés, qui lui sont nécessaires pour les différens usagges auxquels on le destine comme nous le verrons par la suite.

Mais susperidu au plancher.

L'épi dı maiss, privé d'une partie de sa robe, doit être rémni avec d'autres en paquetsde huit on dix, au moyen des feuilles que l'on a dù conserver à l'épi pour cet usage. On suspend alors ces paquets dans les greniers ou sous les hangars, préférablement du côté du midi, en les lixant le long d'une perche ou d'une corde que l'on peut tendre horizontalement ou replier en forme d'anneau. On les suspend alors aux solives du grenier ot on les place à des distances convenables pour ne pas causer d'embarras.

On peut encore les suspeudre sous des avan- 
ces de toits ou sous des portes, el généralement partout où ils peuvent être exposés à des courans d'air, mais cependant il faut les abriter un peu de la pluie.

\section{Mais répandu dans le grenier.}

Si les épis ne sont pas très secs et qu'ils ne soient pas tout à fait parvenus à maturité, on peut encore les étendre dans des greniers à claire-voie, ou, ce qui est plus facile, sur des claies, que l'on suspend au plafond au moyen de cordes fixées aux angles.

\section{Séchoirs à mats.}

Quand on cultive le maïs en grand, on s'y prend ainsi qu'il suit dans les colonies anglaises de l'Amérique. On construit en plein air, mais à portée des habitations, de petits bàtimens qui, au lieu de murs, sont entourés de lattes et même souvent de perches. Elles ne sont pas plus rapprochées qu'il ne faut pour empêcher le maïs de passer dans les intervalles. On leur donne la hauteur et la longueur que l'on veut. Ceux 
des colons amglatis ont ordinainement 17 i is pieds de lomgr. On ne doit leme dommes que 2 i 5 pieds de lamgenr ; s’ils cinient phus laryges, l'air ne: pourrait pas jonce ant llatrers, ef le hul serait manquir. Le toil yui doit pournir s'enterer on se somlever, pour pouvoir y introduire le maïs, est ordinairement de planches posces les unes sur les autres. Le plancher est cigalement en lattes; il est a 5 pieds au dessus dusol, en partie pour que le tout soit plus exposé à l'air, en partie pour yiaratir la récolte des souris et des rats. Cente élévation seule ne suffit eependant pas pour garantir le mails contre ces animanx, si l'on n'adapte pas aux quatre piliers yui soudiennent le magasin, à environ un pied du planchrer et horizontalement, des plateaux ronds en fonte, en tôle on en bois, reconverts en dessous de fer-blanc mince. Ces plateanx doivent avoir assez de diamcure pour empeccher les mats d'attcindre leurs hords. Il sera bon de faire attention de cloner les lattes en dedans de l'encadrement, car autremsut le poids de la récolte pourrait les ditacher. Pour éconoiniser le ferrenent d'une on de plusiens portes 


\section{$(168)$}

par lesquelles on viderait le magasin, on peut, dans ce but, clouer les lattes du plancher à l'extérieur, pour pouvoir les enlever ensuite au besoin (1).

\section{Séchoir de M. de Méja.}

Enfermer le plus de maïs possible dans le moindre espace, l'y dessécher convenablement et l'y conserver à l'abri de l'humidité, de la.moisissure et de l'incursion des rats, tel est le triple avantage de cette méthode, qui, certes, plus qu'ancune autre, mérite d'être adoptée daus toutes les parties de la France. L'idée et l'exécution de ce procédé apparticment à M. de Méja, propriétaire dans le département de la Haute-Garonne.

Ce vénérable praticien a fait construire une cage de la consistance de 600 hectolitres ( 385 setiers) de maïs en épis dans une cour intérieure, et à l'angle de deux bâtimens, l'un à l'ouest et l'autre au nord. La cage forme un cube de 4 mètres $8_{7}$ centimètres ( 15 pieds),

(1) Annales de Foville, Volivraison, p. 32 fel 325 


\section{$(169)$}

sur 3 metres $5 / 1$ combimitres ( 10 pieds) do largeur et atutant de hantemr sur les sablicmers. Eille est chablie sur un plancher ì claire-voie, ileve de 2 midres 59 centimidres (8 pieds), au dessus du sol, supporti par des pontres qui apprient, d’un còte, sur le mur, et de l'autr' sur de's piliers en maconnerie. La cage est reconverte par un toit ordinaire qui est le prolongement des wits voisins.

Son dévation en pente au dessus des satblicres augmente la capaciti de la cage, si on reut lit remplir jusque sous les faites: les còtés dı levant "l du midi sont fermés par des linteaux qui, comme cenx du plancher infirieur, ont 27 millimétres (1 ponce) d'epaisseur sur autant de large, et sont entreux il 27 millimetres d'intervalle. Ce plancher inferieur établi sur de fortes solives, el colles-ci sur des poutres, est d'ailleurs peré à trois endroits les plus distaus des eòtés latcianx de la cage, pour y établir des ventouses qui consistent en trois prismes yuadiangulaires de 65 centimedres (2 pieds) de còtc, fermis parr des linteanx à 27 millimetres de distance les uns des autres, de 


$$
(170)
$$

pareilles largeur et épaisseur, et de la longueu. qui est à peu près la hauteur de la cage. Ces ventouses prennent par dessous la cage l'air extérieur et le distribuent dans la masse du maïs contenu dans la cage.

Depuis plus de trente ans, M. de Méja fait usage de cette cage à mais, toujours avec le plus heureux succès. Sans doute, une expérience aussi longue doit suffire pour prouver qu'il est des moyens de conserver le maïs fort long-temps et dans toute la plénitude de sa bonté.

Sans doute encore, cette méthode sera adoptée dans d'autres lieux. Il faut y faire quelques modifications, par exemple mettre la cage à l'abri de l'humidité et de la pluie, en la fermant en planches minces, qui se reconvriraient l'une l'autre, ou en linteaux disposés en forme d'abat-jour qui rejetteraient l'eau. Quoi qu'il en soit, l'avantage de ce procédi doit déterminer le propriétaire dont les terres sont propres à la culture de cette plante i construirc de pareilles cages à maïs. Déjà nombre de cultivaleuss du midi ont adopté la méthode de M. de Méja et s'en tromrent fort bien. 
Séchoir de M. Lelirur(1).

La construction de ce grenier est simple et peu conteuse, le maïs peut s'y conserver phusieurs amnées. Les solives ont 3 ponces environ et les chevons 2 ponces carrés. Les supports sont formís avee des lattes en chêne de 2 pouces de large sur 6 liģnes d'épaisseur, cloućes a un pouce et demi l'une de l'autre. Les quatre còtés du grrenier sont lermés parr des lattes semblables.

Cette espece de eage est posée sur autant de poteaux qu'il y a de travées. Ces poteaux doivent ètre assez élevés pour que les rats me puissent sauter daus le grenier. Plusienrs plaques de fer-blane faisant saillies sont mises entre la cagre et les poteaux, alin que les rats et les souris qui monteraient aux poteanx me puissent entrer daus le grenier : il serait bien que ces plaques finssent peintes of vernies. Il fant que ce grenier soit isoli, placé dans un rndmit où l'air puisse cirçuler

(1) Ouvrage cite 


\section{$(172)$}

librement tout autour. Le còté de la porte est tourué à l'ouest; une petite porte est placée au dessus de la grande pour achever de remplir le grrenier. Ce séchoir a, comme l'on voit, beaucoup de rapport avec celui employé dans les colonies anglaises.

Séchoir de M. Mathieu de Dombasle.

La planche VI de la septième livraison représente une travée de la cage à maïs, construite à Roville, ainsi que dı hangar dont elle fait partie; cette travée forme le quart de la longueur et de la construction.

Daus la cagge, les épis sont placés en une masse haute et étroite, dans laquelle la circulation de l'air s'opère librement, parce qu'étant entourée d'air libre de tous les côtés, de quelque point que vienne le vent, l'air traverse facilement cette masse, au moyen des interstices que laissent entr'eux les épis entassés pèle-mêle dans la cage. Je n'ai pas osé domner plus d'un mètre de largeur à la cagge, de peur que l'air ne circulàt pas assez librement diuns le milien de la masse; cependant 


\section{$(17)$}

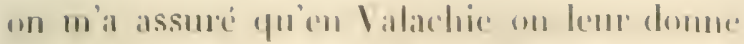

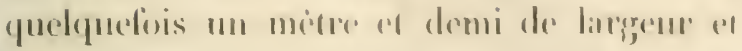
plus.

Celle que jai lait construine at un merre de largenr sur 2 méres de hautem el une longueur indefinie; car, comme je l'ai fai établir an dessus d'un hangar heauconp plus long que la cage dont jai cu besoin, je ne l'ai fait garnir de lattes que sur une partie de sa longueur ( 8 mètres miron). Tomes les fois que les épis itaient passablement mùrs, c'est à dire que les grains étaient durs, quoique la raipe füt eneore tendre et humide intirieurement, ils se sont parfaitement bien conservés, et forsqu'on les a tirés de la cage quelques mois après, ils étaient complétement dessiehcis et exempts de tonte esprice d'altiration. Lorsque la masse contenait, par l'effer de la nigligenee des ourriers, quelques épis mal murs, donn les grains étaient tendres of d'un jaune pàle, ces épis ont ité attaqués de moisissure, mais ur l'out mullement communiquéc à cenx avec lespucls ils étaient en conlact immediat : 'n sorte fu'on pourrait les siparer de la masse anssi facilement qu'on 


\section{(174)}

eù pu le faire au moment de l'emmagasinement.

D'après ce résultat, obtenu dans le nord de la France et dans une localité que l'on peut considérer comme placée sur les limites de la culture du maïs, je pense que l'on peut, avec sécurité, employer ce mode de conservation partout où cette plante est cultivée (1).

\section{Maïs séché au soleil.}

Cette pratique, que l'on peut bien recommander d'une manière générale, n'est guı̀re applicable qu'aux pays méridionaux, parce qu’à l'époque de la récolte le soleil a encore assez de force pour dessécher les épis et que l'on a moins à craindre les pluies, les brouillards, qui pourraient plutôt pourrir le grain que le sécher.

\section{Maïs séché à l'étuse.}

Ce procédé, qui ne pourrait s'employer que pour les petites récoltes, pourrait peut-être

(1) Annales ele Roville, VII livaison, P. $35 \mathrm{r}$. 


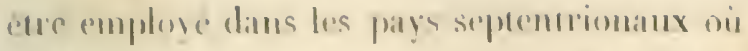
le soleil nese phus assoe fort ant moment de la recolte pour opérer la dessication.

\section{Maïs seche atu four.}

C.est surtout dans le nord que l'on a quelyurfois recours a la chalem du four. Le maïs siche par ce moyen est moins attaquable pas les insectes, il se conserve aussi plus longremps sans altération; mais la ficullé germinative se trouve affaiblic on mime enticrement perduc. Il ne faut donc pas soumettre à ce mode de dessiccation le mais que l'on veut employer en semence. La farine de ee grain est anssi moins prople à faire du pain e) quelques antres alimens anxpuels on fait subir un certain degrei de fermentation.

Pour distinguer la farine du mais séche fe cetle maniere dave elle du mais non scehe an four, on lui donne le nom de grumde. yni est anssi celuigur l'on donue à la bouillie quion en fait.

Le procede usite ru Bourgoerne pour secher le mais au four est celui-ci : 


$$
(1,-6)
$$

On distribue les épis destinés à la fommè dans des corbeilles, puis on chauffe le four jusquau blanc parfait, cest à dire un peu plus que pour la cuisson du gros pain. Le four une fois chauffé, on le nettoie, on y jette les épis que lon étend arec un fourgon de fer recourbé, on ferme le four aussitôt. U'ne heure après, on le débouche, et an moven de la pelle de fer on a soin de remuer le fond du four, de soulerer les épis, de renrerser ceux qui posent sur l’àtre.

On remue les épis une seconde fois, et c'est ì peu près l'affaire de vingt-quatre heures pour compléter la dessiccation du maïs, jusquà ce que le four soit rempli aux deux tiers.

On met les épis, au sortir du four, dans des mannes.

Parmentier conseille de fermer entièrement la bouche du four, et de mettre de la braise derant lourerture.

Un cultivateur de Bourgogne, de quijai obtenu de bons renseignemens sur cette dessiccation, conseille $1^{\circ}$ de ne pas trop chanffer le four, pour que la farine ne devienne pas brume; $2^{\circ}$ de ne pas fermer hermétiquement le four, 


\section{(177)}

pour que l'eraporation de son humidié puisse se: faire plus aisément.

\section{Mais séché à la fumée.}

M. Leblond, dans un mémoire lı à la Société d'agrieulture de Paris, le 15 juillet 1786 , et inséré dans l'ourragge de M. de Neufehàteau, page 155 , indique un procédé suivi par las grands propriétaires de l'A mérique espagnole. Ils niettent leur mais dans une étuve, dont le bas est terminc par un plancher fait d'un bois solide conme le ehène, et à la distance de 2. pieds du sol, on étend des claies sur ce plancher, pour empécher les épis de tomber" on y jette les épis déponillés de leurs euveloppes, à mesure que la récolte s'en fait, par une ourerture pratiquée à qquelqu'endroit de la partie la plus élerée de l'stuve; il doit y avoir une fenctre à coulisse, à quelque distance du plaucher, pour retirer plus commodément le maïs quand il en est besoin. On fait aussi dans le sol une fosse d'un pied au plus de. jrofondeur, qui, de l'entrée du foyer, s'étend 


\section{$(178)$}

assez avant pour pouvoir allumer to feu sous le milieu du plancher de l'éluve.

Un feu modéré, entretenu tous les jours, matin et soir, pendant deux on trois heures, au foyer de cette étuve, suffit pour sécher tout le maïs qui y est contemu. On a soin de laisser la fenêtre supérieure ouverte, pour favoriser l'évaporation de la fumée et de l'humidité surabondantes, aussi long-temps que le maïs en a besoin ; mais quand il est assez sec, on ferme cette fenêtre; on bouche exactement tous les trous qui pourraient donner issue à la fumée, laquelle, ne pouvant s'échapper nulle part, regorge bientôt par l'ouverture du foyer, ce qui indique qu'il faut discontinuer le fen et houcher cette dernière ouverture. Cette fumée, ainsi retenue, devient l'atmosphère dans laquelle le maïs se troure plongé; on la renouvellede temps en temps arec de la paille, lorsque l'on présume qu'il s'en est évaporé. On pourrait éviter la dépense de cette étuve et du feu qu'il y faut entretenir, au moyen d'une séparation qu'on ferait à la partie du grenier où répond le foyer de la maison, et de quelques trous pratiqués à la cheminée, avec des tuiles creuses 
posées diagonalement, de manière que la firmées'en ćchappàl facilement pour se porter i travers le maïs. I a fumér du feu habituel de: la maison suflirait pour gamantir le mais des animaux el des inseetes.

Cefle facon de conserver le mais est sime et peu contense.

\section{Conservation du mais en grain.}

En Italie el en Amérique, où le grain a atteint, lors de sa rícolte, sa maturité complète, et pour ainsi dire l'étal de dessiccation nécessaire à sa conservation, le maïs peut ètre égrené presqu'aussitót qu'il est récolté; mais dans les pays plus an nord, ce grain ne pourrait subir cette opération sans une espèce de préparation préalable, qui consiste à le faire sécher par m des procédés que nous venous d'indiquer, qui ont d'ailleurs tous leurs avantages et leurs inconvéniens. C'est au propriétaire a juger queis sont ceux qui conviennemt mieux à son exploitation, à sa fortune et à son enplacement.

Lorsqu'on se propose de conserver les grains 


\section{$(180)$}

long-temps, voici les règles qu'il faut suivre:

$\mathrm{I}^{\circ}$. On prend des grains récoltés dans un terrain sec et sablonneux et non sur un sol humide.

$2^{\circ}$. Les grains des champs médiocrement fumés doivent être préférés à ceux d'un terrain qui l'a été abondamment.

$3^{\circ}$. On ne met en magasin que les récoltes des années de sécheresse et non celles des années pluvieuses, froides et brumeuses.

$4^{\circ}$. On évite, autant que possible, de mêler les grains des diverses récoltes et des divers terroirs; la simple réflexion que ces grains peuvent être de moins bonne qualité que les autres indique déjà cette mesure de précaution.

\section{Egrenage des épis.}

Gothard (1) aflirme que l'egrenage se fait plus facilement lorsqu'on vient d'apporter les épis du grrenier que lorsqu'ils sont restés seulement pendant une demi-heure dans une chambre chauffée, parce qu'ils commencent à suer et s'attachent plus fortement à la fusée. Aussi conseille-t-il de mettre les paniers qui

(1) Ouvage en allemand, 1797, in-12. 


\section{$(181)$}

les contiennent pendant quelques jours derrière le poèle, ee qui lacilite branconp celle opcration, parce que les épis se sichent totalement. On voit que Gothard ne parle ici que des épis suspendus à des perches ou étendus sur des chaies, car pour ceux sćchés au four à la fumce ou à l'étuve, cette précaution est inutile.

Maintenant que nous conmaissons les différentes manières de sécher le grain, condition nécessaire pour pouvoir l'égrener, nous alIons décrire les différens proecédés usités pour celte operation.

I $^{\circ}$ 'A la main. - Le plus souvent on laisse les épis entiers pour les égrener à la main; mais dans quelques endroits, par exemple dans quelyues contrées de l'Allemagne, on les rompt en deux ou trois portions.

$2^{\circ}$. Par le frottement. - On frotte deux épis l'un contre l'antre; mais alor's l'épi qu'on veut dépouiller reste immobile dans la main gauche, tandis que la droite tient celui qui sert d'instrument. Cédernier sedéponille aussi, et le corps qui reste s'use ensuite plus ou moins promptement, parce qu’il est d'une substance médullaire. 


$$
(182)
$$

$3^{\circ}$. Avec une faucille. - On se sert anssi d'une faucille ou de tout antre instrument de fer, comme une queue de poêle par exemple; on prend l'épi de la main gauche, le tenant par un bout perpendiculairement sur un morceau de planché; puis on fait passer l'instrument le long de l'épi.

$4^{\circ}$. Sur un tomberecuu. - Un se sert d'un tombereau soutenu par quatre pieds, percé dans tout son intérieur de trous par où les grains, détachés de leurs alvéoles, puissent passer. On établit ce tombereau au milieu du grenier; on y met une certaine quantité d'épis. Deux hommes placés aux deux extrémités frappent dessus avec des bâtons; on repasse ensuite les épis à la main, pour en séparer les grains qui peuvent encore y être restés. Cette facon est particulièrement usitée dans le pays de Navarre, où le maiis, assez ordinairement fort sec, ne demande qu'une compression peu forte pour se détacher aisément.

Le procédé mis en usage dans la province des mines (Brésil) est à peu près le même.

"Très souvent on égrène le mais; mais dans les habitations un peu considérables, on 
le bat sur une machine quion appelle batedor 't qui est construtro comme je rais l'expliquer en peu de mots. Entre quatre grands poteans d'envirno 6 pieds, on élablit, à la hantem de 5011 á pieds, quatre pieces de hois transtersales el tris lores, qui forment $\| 1$ carré de í i 5 pieds. Sur deux de ees piéces de bois, on place parallèlement des bitons arrondis de la grossem du bras, en ne. laissant entreux qu'un intervalle de 5 à 6 ligunes, el on garnit d'une natte verticale trois des cotés de la machine qui ne reste onverte que pat devant. Quand on vent battre dumaïs, on entasse des épis jusqu’à la hauteur d'un demi-pied, sur l'espéce de table ou de claie formée par les bàtons transversatux du butedor, et des niggres frappent sur ces épis avec de longs bitons. Lat natte verticale retient les épis qui pourraient s'icarter ; les grains, détachés de leur axe, passent à travers les barreaux de la claic, et ils tombent sur un cuir qui est placé au dessous (1). "

(1) Tojage dans l'intericur du birésil, par tug. de Saint-Hilaire, 1. I, p. 234, 183o, in-8o. 


\section{$(184)$}

5․ Avec le fléau. - On bat les épis renfermés dans des sacs ou à nu avec un fléau. Ce procédé, qui est très expéditif, s'emploie souvent lorsqu'on a de fortes récoltes; mais si le grain est trop sec, il se brise souvent sur le coup et ne peut plus servir à l'ensemencement. Cette méthode est presque la seule employée par les paysans du royaume LombardoVénitien.

$6^{\circ}$. Sur une barre de fer. - Dans le département de la Haute-Garonne, on se sert d'une barre de fer de 3 pieds de long, sur une épaisseur de 4 lignes et une largeur d'un pouce. Elle est arrêtée par les deux extrémités à deux escabeaux, sur lesquels deux égreneurs travaillent assis l'un vis à vis de l'autre; ils prennent l'épi de la main droite, le raclent fortement en remontant contre un angle de la barre, en l'accompagnant par dessous des bouts des doigts de la main gauche, avec laquelle ils empoignent le fer. Dans deux coups de main, la fusée est dépouillée; alors ils prennent l'épi par cette partie dont le grain a été séparé, et en deux autres tours de main ils égrènent le reste; la fusée reste entière. 
Le procede de M. Jacques, jardinier en chef du donaine de Nenilly, a beanconp d'at nalogie avec celui-ci.

L'instrument dont il se sert consiste en une lame concave, embrassant le liers ou la moitic de l'épi qu'on fail glisser dessus.

$7^{n}$. Sur une mesure. - Un instrument tres commode pour cette opération, parce qu'il sert en même tempss de recipient pour les grains 'qui se détachent, est une mesure de capacite pour les denrées, ayaut la forme d'un eylindre plus large que haut.

A la surface se trouve une tringle de fer aplatie, qui divise l'orifice de la mesure en denx parties égales, en s'étendant d'un point de lia circonférence au point opposé. Au centre, elle est appurece sur un support vertical, fixi d'un bont an fond de la mesure, cit de l'autre a la tringle. On se sert de cet instrument comme de la base de fer dans le sixiene procedé.

S". Avere la filière. - Je sais, dit M. Romand (1), que, dins plusiens départemens ou

(1) Fenille du cullivateur, 5-6, 1. VII, 1' partie 1. 83. Paris, an VI de la république, in-4n" 
on cultive le maïs, on commence à détacher avec les doigts quelques grains du laut de la fusée; ensuite, avec deux morceaux de bois qui se croisent, on en détache ie grain avec peine; la machine que je présente, simple par elle-même, a l'avantage d'accélérer l'ouvrage sans ce préliminaire, et la fusée est parfaitement dépouillée de son grain. J'ai nomnié eette machine filière par la forme de sa composition : ainsi, avec cette filière un homme peut, dans un jour, détacher un setier el plus de grains, ce qui équivaut au moins à ce que fait de travail un batteur en grange. Ce travail peut se faire à la veillée; huit heures ont suffi pour me donner dix boisseaux de ce grain. Il s'agit de tenir d'une main la filière et de l'autre une fusée, la présenter au centre en la tournant uin peu obliquement à droite et à ganche sans que la main qui tient la filière gène les mouremens des ressorts qui doivent obéir suivant la grosseur de la fusée.

Le maïs égrené occupe la moitié du volume qu’il occupait en épi.

9\%. Aree la machine de M. Fol. - Cetto nachine, qui a été inventie par M. Fol, di- 


$$
\left({ }^{8} 8_{7}\right)
$$

recteur de la fousterie de Babialau, a Bordeaux, se recommande par sa simplicite et far les resultats heureux el prompts quon en retire. En effet, il suflit do mettre l'épi dans une espëce d'entonnoir, et de donner ì la machine quelques tours de mue, pour obtenir le grain scipare de la fuséc qui reste encière. Cet instrument est employé par heaucoup' de grands cultivateurs du midi de la France. II. Bossange m’a communique le dessin qui Ini a été envoyé de Bordeaux; on le trouvera reproduit à lis fin de cet ourrage. (Voir la planche II.)

D'après des expériences nombreuses, il parail que le mais perd bien moins de son poids à n’ètre séparé de son épi que quelques mois apres sa ricolte, que lorsqu'il est igrené à peine rentré ì la maison. Lorsquion a séparé, par un procédé queleonque, lè grain de la tige i layurlle il ctait adherent, il reste le papeton sur les usages duquel nous reviendrons plus tord.

$$
\text { Mais ell tas. }
$$

Lorsque le maiss an regrence, nn le porte 
quelquefois au grenier avec son enveloppe, nommée ballot en Bourgogne, enveloppe que l'on prétend bonne à entretenir autour dı grain un froid avantageux et propre à lui conserver son goût de fruit ; mais le plus souvent on le vanne et on le dispose par tas, pour s'en servir au besoin. Ces tas ne doivent pas être trop gros, parce que, quoiqu'il paraisse see, le maïs conserve encore quelquefois beaucoup d'humidité, qui, lorsqu'il est amoncelé, le fait fermenter et le corrompt; ces tas ne doivent pas avoir, la première année, plus de 4 à 5 pouces de hauteur. Pendant l'hiver, on les remue quatre ou cing fois avec la pelle de bois, en tenant toujours les fenêtres bien closes; on ne les ouvre que lorsque l'air commence à devenir rare. Aussitôt l'été, it faut les remuer au moins une fois par semaine, et ouvìir de temps à autre; mais en automne, on doit clore de nouveau les fenêtres du grenier, afin que l'air humide et le froid n'y pénètrent pas.

La deuxième année, après l'avoir remué à la pelle, on rémit plusieurs tas, auxquels on dounc plus de hauteur, et on vanne le grain 


\section{$(189)$}

an moins tous les puinze jours. En remuant sonvent le maiss on l'empeche de fermenter, et on chasse plus ficilement les insectes pernicieux qui s'y logent. Ces différentes manipulations se multiplieraient beanconp moins si le grenier était établi dans un emplacement see et at une exposition libre. Ainsi, il conviendrait que l'exposition du batinent fùt an nord et à l'ombre, dans le voisinage de rochers, de maisons, ete., qui peurent répercuter la chalenr. Il est essentiel d'en garantir le magasin par un triple ridean de pins, de sapins ou de peupliers.

Si l'on ne peut réunir ces conditions, il faut au moins ménager un courant d'air artificiel.

On pent r'mployer it eet usage le ventilatem. de Duhamel. Cet agronome distingue, croyant nécessaire de ponvoir conserver une grande quantité de gyrains dans un petit espace et aree le moins de dépense possible, imagyina d'adapter au grenier son ventilateur. Il proposa done de disposer le grain dans des cases de bois dans lesquelles l'air pourrait être agyité par le ventiliteur, par le moyen de beaucoup' 


\section{(190)}

de trous qu'on y pratiquerait et qui emporteraient l'humidité. Le maïs peut se mettre dans les cases à telle hauteur qu'on veut.

En fait, une case de ro pieds carrés peut contenir r,ooo pieds cubes de grains, tandis qu'une quantité égale, étendue librement dans un grenier, occuperait un espace de 58 pieds de longueur. De cette manière, on ne doit pas craindre la fermentation, mais seulement le dégàt causé par les insectes.

\section{Maïs en sacs isolés.}

Parmentier ( page $\mathbf{6} 6$ ) conseille, pour garantir le maïs de l'humidité et des animaux destructeurs, de le renfermer dans des saes, en évitant de placer ces sacs auprès des murs ou de les entasser les uns sur.les autres, en les isolant autant que l'on peut, afin que l'air ambiant puisse circuler tout autour et les traverser dans leur épaisseur. C'est, dit-il, un bon moyen pour garantir ce grain de la poussière, des ravages des chats, des rats et autres animaux destrueteurs. 
Mats dems des forseses on silos.

L'usage de conserver les hlés dans des fosse's ou silos date des temps les phus reculés; elles les garantissent du pillage.

Dans les contrées où le despotisme n’a aucun respect pour la propricte, en Turquie parexemple, les habitans enfouissent leurs grains, pour les meture à l'abri de la rapacité des gouvernans.

Cette méthode est usitée en Russie, en P'ologne, en Egyptr, en Sicile, en Italie, anx Indes-Orientales, "t dans beaucoup d'antres contrées de l'Orient.

Dans les paỵs Barbaresques, on dépose le blé dans des fosses crensées dans lo roc, ì 3o, 40 et même So pieds de profondeur, dont l'étroite onverture est bouchíe par une espèce de claie converte par ? pieds de sable: en sorte qu'on fait jasser lat charrue par dessus. L'intérieur est garni de planches et de nattes, qu'on revè encore de paille. Ces fosses s'appellent matadores, le blés'y conserve durant quinze années. 


\section{( 192)}

Au royaumede Naples, on les appelle foggin; elles ont donné leur nom à la eapitale de la Ponille.

II y en a de tellement vastes, qu'elles peuvent contenir 2,00o muids de blé.

En Italie, on sème à la surface des fosses de la ehaux vive, qui se dureit à l'air et forme une couche impénétrable.

Les ruines d'Hereulanum, qui fut englouti l'an 79 de l'ère chrétienne sous les laves du Vésuve, nous ont fourni un exemple frappant de la prodigieuse vieillesse que peuvent atteindre, sans se décomposer, les blés enfouis dans le sein de la terre; car lorsqu'en 1738 ( 1), on les fouilla, on découvrit, entr'autres choses, sous les cendres et les débris des substances calcinces, des grains parfaitement conservés.

On voit encore aujourd'hui, à Amboise, les rnines des grreniers de Jules-César, consistant en trois grrandes galeries, taillées en voûte dans le roc, où il avait fait pratiquer des silos.

(1) Un paysan découvrit cette ville, en i 7 I , en revis'mt un puits. 
Ce procédétail dejà comm anx Amérieans avant l'arrive des Enoprens ; rar on lit, dans Warden (1), quils cremsaient des trous daus 11 chchoit tres sec, sur le penchant d'mne colline, les garmissaient de natles et y déposaient leurs ricolı's de maïs.

Si la honche du tron est faite de manière que l'air ni l’humidicé no puissent y pénétrer, on ne doit erandre ancun dommage pour ce frain. C'est surtout pendant la gruere que cette méthode est adoptíe.

Un chef de famille a plusicurs fosses de cette espece, afin que si l'ennemi en díconve une, les autres puissent être sauvées. Elles servent encore quand une famille va chasser pendant six mois; le mais s'y conserve tres hien, soit en épi, soit en grain, pendant plusicurs années.

C'est le mème procédé que l'on suit en Afrique et en Espagne; quelquefois cependant ces fosses sont revêtues, au lieu de paille, d'écorces ou d'herbes sèches.

(1) Recherches sur les antiquités de l'Amérique sepentrionale, par Warden, p. 102. Paris, 1827 , in- $4^{\circ}$. 


\section{( 194$)$}

Varennes de Fenille assure que, par ce moyen, le froment peut se conserver cinquante ans et le millet cent. Comme le mais a quelque ressemblance arec le millet, on peut croire qu'il s'y grarderait long-temps.

Parmentier indique, dans une note, page 102 , comment les paysans de la Basse-Égypte s'y prennent pour le conserver. Leur chaumière n'ayant jamais que le rez-de-chaussće, ils en bàtissent une antre exprès, à côté, dont les murs ne sont que de terre pétrie et séchée au soleil; on les remplit par en haut, et lorsqu'elles sont pleines on les couvre et on y met ensuite une conche de la même terre. Au bas de l'un des côlés, on pratique me porte de a pieds, par où l'on retire la provision de chaque semaine. Dans la Hante-Egypte, on fait des citernes an milien de la chanmière; elles servent de grenier, non seulement pour le maïs, mais encore pour le. froment et les autres grains.

En suivant l'impulsion donnée par les autres peuples et l'exemple donné par M. le baron Termaux, qui a fait construire des silos dans sa propriété de Saint-Ouen près Paris, où il conserve amnuellement une grande quantité de 


$$
(195)
$$

froment, on pommit, creme semble, emonrager ce mode de conservation en l'appliquan an maïs, en faisant faire des silos pareils ì relui que l'on voit figyrure dans la brochure du même reonomiste, intiuler : Mémnise sur la ronservation des greains dams les silos nu fosses souterminus, 1825.

Divers alutes moyens de consenvation.

Toici un procéde grénéralement mis en usigge par les paysans de Pologne et dont les Chinois font généralement usage pour conserver leurs grrains.

Il consiste dans de grands paniers en paille, auxquels on peut donner jusqu’à me dimension de 2 mètres sur r2 décimétres de diamètre. Ces especes de paniers on tomneaux sont formés avec de la paille que l'on dispose en forme de corde, sans cependant la tordre. Ces cordes, qui peuvent avoir z pouces de diametre ou de grosseur totale, se fabriquent en prenant, par exemple, une poignée de paille la plus longue et la plus séche que l'on peut trouver, que l'on serre avec de la fieclle 


\section{$\left(19^{6}\right)$}

mise à une certaine distance, pour qu'elle acquière une certaine longueur. On roule cette corde à peu près comme une bouggie ordinaire, et on la maintient dans cette position en cousant ces spirales ou révolutions de cordes les unes avec les autres, à peu près comme une natte. Il esı aisé de concevoir qu'on doit avoir soin de mettre la nouvelle paille un peu avant que la première finisse et de l'entrelacer de manière qu'il ne puisse pas se faire une solution de continuité.

Cette façon d'entrelacer les brins de paille est aisée : elle ne consiste qu'à en mettre de nouvelle au dernier quart de la longueur de celle qu'on a déjà employée, et de n'approcher la ficelle que dans les endroits où l'on fait cetteaddition. Les tonneaux, à qui on donne ordinairément 4 pieds de haut sur 3 de diamètre, étant finis, on les remplit de grains bien vannés, dans un temps see, qu'on couvre avec un peu de paille, et l'on met le couvercle que l'on coud avec les bords du tonneau. L'expérience prouve que le grain s'y conserve parfaitcment, pourvu que les tonneaux soient à l'abri de la pluie. 


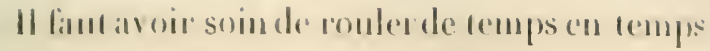
ces tomncaux. Le diplatement des graims que refte manouvre oceasione produit à pen pres le mème effer que si on les remuait à la pelle.

On a encore proposé les fommeanx gourdronnés a l’intéricur.

M. Arsenne Thiebaut de Berneand dit. avoir vu et mangé de la farine de mais qui avail quatre ans de tonnean; elle avait été apportice, en Italie, des États-Unis. Francklin avait aussi proposci, pour conserver les farines sur mer, principalement les tomneaux et barriques doublés de plomb laminé.

Les Allemands conservaient antrefois leurs grains an moyen d'un mortier qui les enve loppait.

On pourait encore les sommetre, dams les greniers, à des fumigations sulfureuses ou nitreuses, qui feraicut périr les insectes sans faire ancmu tort aux grains.

Tous ces moyens peurent ère, ou semls ou combinés ensemble, appliqués au maïs. 


\section{Mouture des grains.}

Le gyrain de maïs doit ètre tout à fait privé de son humidité lorsqu'il s'agit de le moudre, il ne faut pourtant pas qu'il soit trop sec; il faut éviter aussi de le faire moudre conjointement arec d'autres grains, quand bien même on voudrait mèler leurs farines, il faudrait attendre qu'elles aient été toutes moulues à part pour les réunir ensuite.

Lorsque le mais a été suffisamment trituré, il rend plus des trois quarts de son poids de farine et le reste en son. Il est très essentiel d'observer que, lorsque les farines de maïs sont moulues trop fin, elles perdent leur qualité. Il fant qu'elles conservent toujours un grumeau fin, ou autrement le parfum s'évapore, et elles deviennent pâteuses comme la farine de froment. J'indiquerai plus tard comment on doit faire pour obtenir une farine phus fine en la tamisant et faisant servir ces grumeaux pour la semoule. Il n'est pas moins important de saroir que les gुrains dont on retire la farine que 1 'on destine pour les gau- 


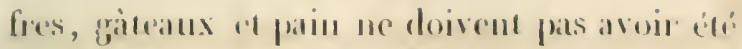
seches an four : cern, an contraire, qui doirent dommer colle qui servina pon les gandes, la polenta doivent aroir suli celle opération. Je remvoie eenx qui voudront des ditails sur la montume de ee grain a la page 170 de l'onvarge de Parmentier, qui a fait, à cessujer, de belles el nombeuses expériences.

\section{Levosement des gratus.}

Pour faire servir le mais à la nomriture ordinaire des hommes, on le prépare de deux manières différentes dans la province des mincs (Brésil).

Sa farine, simplement moulue el séparéc du sou, à l'aided'un tam is de hambou, prend lenom de fubri. C'est en faisant bumillir le fului dans de' l'eitu, sans y ajouter de sel, que l'on fail celle 'spéce de polenta grossicre qu'on appelle angui, "t qui forme le principal aliment des eselaves.

La firụe dont se nourrissent généralenent les hommes libres exige quelyues prépara-lions de plus. On sipare le maïs de ses cuveloppes à l'aide de la machime quion nommone 
manjolı. La manjola se nomme aussi préguiça. Sur une pièce de bois verticale et inmobile, est placée, à la manière d'une balancoire, une autre pièce de bois mobile et horizontale. Cette dernière est creusée, à une de ses extrénités, comme une large cuiller, et à l'autre bout elle est armée d'une dent très forte. La machine est toujours placée au dessous d'une petite chute d'ear. Le liquide, en tombant dans l'espéce de cuiller qui, d'un côté, termine la pièce de bois, fait pencher celle-ci de ce même côté, tandis que l'autre extrémité, arnée à la face inférieure, d'une dent épaisse et conique, s'élève en décrivant un arc de cercle; mais pendant que l'extrémité creusée s’incline, l'ean s'écoule, le poids de la dent l'emporte sur celui de la cuiller, la machine crie, et la dent tombe lourdement dans une auge destinée à recevoir le grrain. Cette dent dépouille le grain sans le broyer, et un peu d'eau qu'on a eu soin de verser dans l'auge, en facilitant la séparation des enveloppes, empêche encore le grain de santer et de se perdre. Quand le maïs est ainsi nétroyé, on le fait tremper dans d'autres auges dont on tàche que 


\section{(201)}

leall se remomvelle sans resse; on ly latisse pendant deux on trois jours ef meme davanlayge, jusqu'an moment ou la lementation va commencer : alors on le reporte lien imbibe dans la manjolu, et par le mojen de cette matehine on le rédnil en me espece de bonillie. On passe cette derniere a travers un tamis au dessus d'unc chaudière peu profonde, sous laquelle on allume du leu; la prite en bouillie se seche, elle se réduit en une poudre grossiere, et e'est là ce qui forme cette farine (farinha), dont on sampondre, comme je l'ai dit, les alimens, et qui, sans ameun doute, est plus savoureuse et plus nourrissante que celle du manioe. Ces défails emieux sont lirés de l'excellent ouvage de $\mathbf{M}$. Auguste de Saint-Hilaire sur le Brésil.

\section{Farine de maïs en sacis isolés.}

Parmentier dis, pagre 195, "qu'il est anilorisé à croire yue quand ce grain a été récolte sec daus les champs, yu il a ressur an grenice, qu’il a été égrené an temps opportun et momb selon les bons principes, l'expérience, lol, 


\section{$(202)$}

servation et le raisonnement réclament en faveur de sa conservation en farine, refroidie à l'air, renfermée dans des sacs propres, écartés des murs, isolés les uns des autres, et placés dans l'endroit le plus sec du bâtiment. "

Il est constant que la farine de maïs acquiert du poids, à raison du local des surfaces qu'ellı présente et de l'état de I'atmosphère, mais cette alternative d'augmentation on de diminution est moins sensible quand la farine est renfermée dans des sacs disposés et arrangés comme nous venons de l'indiquer.

Si, par quelque circonstance particulière, lis farine de maïs avait contracté quelqu'odeur nauséabonde et qu'on n'eùt pas le temps de l'employer, ou si on voulait la transporter dans un lieu éloigné, il faut avec toute sûreté l'exposer au feu, puisque cet aggent n'altère nullement la farinede maïs, comme il altérerait celle du froment, parce que cette dernière contiènt beacoup de gluten.

Harasti di Buda (1) dit avoir séché de la fa-

(1) Mella collivazione del maiz del padre Gaetann Harasti di Buda, P. gs. Vicenza, i 788 , in-8º 


\section{$(\cdot 05)$}

rine de matis, el l'aroir conservée, pendant de longues années, en suivant l'exemple d'un économe industrieux qui la placait dams mmo chambre sicuce au dessus d'un four.

Les paysans italiens pensent que, pour conserver mu sac de farine de maïs sans qu'ellr prenne de chalem, on n'a qu’à introduire dans le sar mu morceau de fer.

\section{EMPIOI.}

Nourriture des animanx avec le mais.

Citer Parmentier à chaque pas, c'est sans au'un doute rendre hommage aux connaissances précieuses de cet reonome distingué : nous allous lui emprumter encore une partic de cel article $(1)$.

"Il n'y a pas de meilleur yrain que le mais pour en former des prairies artificielles, sa végritation est prompteet la plantefoisome en herbe. Toutes les terres, pourvin qu'elles soicut menhles et quidles aient un peu de fond, lni con-

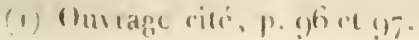




\section{(204)}

viennent. Celles qui ont rapporté du seigle, du lin, de l'orge, de la navette, les jachères elles-mêmes, quoique déjà fumées, pourraient y être consacrées sans nuire aux récoltes futures; car ni le sol ni l'engrais ne sauraient être épuisés par une plante qui y séjourne aussi peu de temps, qu'on se hàte de couper avant la fructification, et dont la racine flexible et succulente, se pourrissant aisément, rend à la terre au moins l'équivalent de ce qu'elle a reçu. Je le répète, le maïs-fourrage réunit le double avantage de plaire à tout le bétail, et de le conserver dans un état de vigueur et de santé, si nécessaire surtout pour le renouvellement de l'espèce. " Le maïs le plus nouveau doit être choisi de préférence pour le semer comme fourrage, parce qu'il lève plus vite et fournit plus ale feuillage. Après avoir donné à la terre un bon labour, on sème ce gurain à la volée, en olsservant que le semeur remplisse bien sa main et raccourcisse son pas; sans cette précaution, le grain, vu son vohume, serait répandu trop clair. On l'enterre avec la charrue ef la herse, qu'on lait passer drux fois en tous sens. Il faut par arpent 


\section{(2.05)}

3 ou 4 hoisseamx de mais, er yui forme d pen pres les mois yuarts en sus de ce qui est nécessaire ordimairement quand il s'agit d'en récolter te grain. Commone les antres gramens somes à dessein d'en récolter le fomrage, l. maïs ne cansi ancun embarras, et sa cultur n'exige point de lavaux, ee qui est doublement avantageux dans une saison où l'habilant de la campagne est le plus oecupé. Le grain, unc fois semé er recourert, est abandonne aux soins de la nature. Il n'a besoin d'ère ni sarclé, ni lnuté, ni ćclairci. Il est imtile d'observer aucuno rérubularité entre les pieds et de s'occuper de la maturité de l'épi, paree que e'est l'herhe seule qu'on veut recueillir.

Le fauchage doit se line deux fois par jour, le matin apres la rosée, le soir mue heme on deux avant le coucher du soleil. Il faut bien se garder de couper le mais en vert au milieu de la journce, surtout lorsque le soleil domine, car il s'echaulfe facilement; dans cet ritat, ou il est refusé par les bestiaux, ou il les expose à Jes maladies.

M. N..., proprichaire el cultivateur en Vendie, repiefur at plante fe maïs tous les mois, 


\section{(206)}

comme fourrage; il en nourrit les veaux de l'année. Dans tous les pays où on. cultive le maïs, on connait l'usage des tiges pour la nourriture des bestiaux, et surtout des vaches, à qui elles fournissent un lait plus abondant. et plus savoureux.

Parmentier dit avoir remarqué que le lait des vaches, dont l'aliment consistait uniquement en fourrage de mais, était plus sucré que celui de ces animaux entretenus avec des herbages ordinaires. M. Bose appuie cette opinion de son expérience. L'usage de cette plante rend raison du bon état où l'on voit le bétail dans le midi de la France, en Espagne et en Italie, dans des situations qui ne semblent pas propres à la culture des prairies ordinaires.

On ne se sert pas seulement des tiges, mais encore des panicules qui les surmontent, des épis et des feuilles surabondans, et que l'on est obligé d'enlever pendant le cour's de la végétation de la plante, pour lui donner plus de force et faciliter la maturité des épis fruitiers. On le leur donne soit vert, soit sec pendant l'hiver. 2 arpens métriques de maïs peuvent, avec cette demière ressource, entretenir 


\section{$(207)$}

"ufourageres verts huil a dis vaches pendant deux mois al demi.

Un arpent cullive comme fonrage peut en donmer roo yuintamx sers. Dans les landes or les départemons de la Hantr-Garomme, les cullivatems estiment que 6 arpens de mais ou Thectares equivalem, par leurs farines, i m arpent on 51 ares de bon pré. Ils domment aux liges privés de leurs ipis le nom de tronisses ou camborles.

Lorsqu'on rem domner le maïs eomme fourlage, on compe ordinarrment les tiges, an moment oi les paniculesdes flenrs màles commoncent à se montrer. Le maïs ainsi coupé se dessèche conme le foin, mais il faut beaucoup plus de temps pour op oprer la parfaite dessiecalion. Le fourrage seconserve bon pendant denx on trois ans.

On peur encore employer à cet usage les rrjetons qui partent des racines. Les chevanx, les mulets, les boenfs, les vachesen sont trèsavides, on les donne encore aux lapins de clapier.

Gothard (1) dit quiil pouvait à peine con-

(1) Curtage cité (en allemand). 
duire sa mule quand il traversait des champs plantés de maïs, parce qu'elle voulait absolument en manger.

La pénurie du fourrage dans les Landes, et peut-être aussi la grosseur des fanes, y ont introduit, nous assure M. De Candolle (I), un usage assez bizarre; c'est que les bœufs n'y mangent pas seuls. Tous les jours, dans chaque ferme, un homme chargé de cet emploi, et nommé emboqueur, donne aux boufs leur nourriture dans la bouche. Ordinairement, à côté du foyer, se trouve une fenêtre qui donne dans l'étable et par laquelle se fait cette opération. Dans les marehés publics, rien n’est plus bizarre que de voir les paysans agenouillés devant leurs boufs, qui reçoivent majestueusement leur nourriture sans se baisser.

Quelques cultivateurs, au licu de donner les tiges entières, les coupent avec le hache-paille et les mêlent avec des pommes de terre décou-

(1) Mémoires de la Socićté d'agriculture de la Seine. Extrait d'un rapport sur un voyage botanique et agronomique dans les départemens du sud-ouest, t. XI, p. 59 . 


\section{(2) (19)}

press, d'antres aree des cillomilles. Tombes les especes de: maïs peurent servir, par lemes granins, à mourrir la volaille yui profite à vure d'eeil avece celte senle nourriture; mais avant de donner ee granin à la jeune volaille, il faut le eoncasser sous la menle. Les clapous de Bresse, qui pésent jusqü: 10 01 12 lives, ue doivent leur réputation qu'au blé de Turquir. La volaille nourrie avec le maïs n'a d'antre inconvénient yne dètre trop grasse. On doit avoir l'attention de changer cetle nourriture an moment de la ponte.

Lat chair des pigeons de volière gu'on en nomrit est blanche, tendre, et leur graisse est ferme et savoureuse.

Depuis le $\mathrm{r}^{\text {er }}$ sejptembre 1829 , jai élevé seulement avec cette nourriture deux pigeons, trois poules et un coq, qui tous mangeaient à peine seuls; its n'ont eu jusqu'en février 1850 d'antre nourriture que du granin de maïs ou de la boüllie faite avec la farine de ce grain. Dans les premiers jours de janvier, m des pigeons fut tuc el pesi aree un desplus beaux pigeons trouvé au marelsi. Le mien pesait tout diponillé /905 grammes, l'autre 500 grrammes. 


\section{(2I1)}

Dans le premicr la chair était extrêmement ferme, les intestins surchargés de graisse. A la cuisson, le pigeon acheté avait diminué de moitié; le mien, au contraire, s'était bien maintenu et avait plus du double de volume que le précédent; le premier n'avait plus aucum goût auprès de mon élève, dont la chair était délicieuse.

La mème expérience, répétée avec le même soin, fut faite toujours comparativement avec mon antre pigeon, mes poules et mon cor; je n'ai plus besoin d'indiquer de quel còté s'est trouvé l'avantage.

Les cochons de Naples, qui pèsent jusqu’à 500 livres, ne sont engraissés qu'arec le grain de maïs ( $\mathrm{I}$ ).

Selon Gothard, près de Debreczin, ville capitale dela Haute-Hongrie, on élève de très beaux porcs, ils ne sont nourris qu'avec ce grain (2).

Il est remarquable que ces animaux ne se dégouttent jamais du maïs, comme cela leur arrive avec des féveroles.

(1) Encyclopédic, article Maïs.

(2) Ouvrage cité ( en allemand). 
A Naples, pour teur douner un- land leerme et cpais, on les enferme simplement, pendan denx mois, dans une logge oil est une ange toujours pleine de re reain, demt ils se rassasient en liberti.

On al essayé, surtomen Amerique, de donner ce yrain aux chevaux en place d'avoine, mais it a $u$ inconvénient grave, c'est fu’il use ef fait remuer leurs dents bien plus tôt que les autres granins, à raison de son extrême dureté. Le moven de parer ì cet ineouvénient serait de ne le leur douner qu'apres vingtquatre henres d'immersion dans l'ean (1). II faut d'abord le mèler avec l'avoine, pour leur en faire prendre le goont. Il serait probablement aussi très avantagenx de le concasser.

Enfin ne fant-il pas que cette nourriture ait beaucoup d'attraits pour quelques animaux, ear on ajoute : Dans le même pays, les chevaux, les valches, les cochons sont laissis libres, tonte lannce dans les hois, et c'est l'appàt d'ume poignére de maïs qui fait ro-

1) Nouveau Cours d'asticulure, p. 28(1). 
venir, tous les soirs, ces animaux au logis (1). ")

Au Brésil, tous les mulets ne sont nourris qu'avec du maïs.

Les chiens destinés à la chasse du cerf sont nourris au Brésil (province des mines) avec de l'angúu ou de la cangica. Ce sont deux préparations très simples de farine de maïs.

Les grains de maïs jetés dans un vivier rendent plus savoureux les poissons qui les mangent (2). On croit généralement que le maïs qui a essuyé quelques gelées est plus profitable aux bestiaux.

Harasti s'est servi du suc des tiges pour. nourrir des abeilles (3). Je pris, dit-il, une ruche peuplée de peu d'abeilles, et dont la quantité de miel contenue était à peine suffisante pour les nourrir, puisqu'elles mouraient presque de faim; je donnais à ces pau-

(1) Nouveau Cours d'agriculture, p. $28 \mathrm{~g}$.

(2) Dictionnaire des sciences naturelles, par les professeurs du Jardin du Roi, édition Levrault, 1. XXVIII, p. 112.

(3) Della collivazione dell' maiz, etc., p. 110 , in-8 . 


\section{$(2.13)$}

vres bêtes me parlic de mon suc ì demi condensé, ce qui sullit pour les faire subsister pendant un hiver entier. La mehe, qui était composée d'insectes faibles, au printemps suivant se trouva peupléc d'abeilles vigoureuses qui mattiplierent a merveille. L'autre portion de suc, réduite en consistance de sirop, servit à me donner, pendant tout l'hiver, une boisson pectorale et vraiment agréable.

La Bibliothéquedes propriétaires ruraux (1) renferme un autre moyen d'employer les lifres; voici ce qu'on y trouve : "Si la paille de blé, indépendamment du lait qu'elledomne, esı encore nourricière, à combien plus forte raison doit l'être celle du maïs, ce canal long et volumineux! Pourquoi, dès actuellement, ne lerait-on pas moudre ces tiges qu'or emploie an chauffage, pour en préparer le pain des chiens de basse-cour, qui pourrait encore servir à la nourriture des pores et de la jeune volaille? On économiserait d'autant le grain destiné à cet usage. Cet emploi des tiges de

(1) Bibliolheqque des propriciaircs ruraux, 1. III, p. 139 el 1 f́o. 


\section{(2.14)}

maïs a été indiqué, il y a plusieur's années, dans un journal de Viemne, par M. l'abbé Fortis. "

\section{Usage économique des tiges.}

Les tiges de maïs peuvent servir à couvrir les toits rustiques, et après plusieurs années de service elles n'en sont que meilleures pour' être converties en engrais.

On dit avoir retiré des tiges de maïs un fil assez fort pour pouvoir servir aux tisserands. Parmentier indique dans son ouvrage, page r 39 , d'en bruiler les tiges, pour en retirer d'abord de la chaleur, $2^{\circ}$ des cendres qui fournissent beaucoup de sels, que l'on peut obtetenir' en les lessivant.

D’après des expériences, 4 40 livres de tiges de maïs fournissent 39 livres de cendres qui, lessivées avec $6 \mathrm{r}_{2}$ livres d'eau, donnent 17 livres 1 ? onces I gros 36 grrains d'alcali.

Les Américains fendent les tiges quand elles sont sèches, les taillent en plusieurs filamens, dont ils font des panier's et des corbeilles de difficentes formes et grandeurs. 


\section{$(2,5)$}

\section{firmilles.}

Amorem, dims te tompte quil rend de son

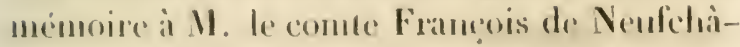
reau (1), dit qu'il proposait l'essai d'un papier. labrigue ane les leuilles de maîs : pourquei, s'erie-t-il, l'art de la corderie ne tireratt-il pas parti de la libasse de ces memes fenilles?

Il propesait encore aux peintres l'emploi à l'huile de la poussicre noire entenue daus lo's tumeurs dont il a cté question, elle lui a parm domerer un moir plus siceatif; il invitaiı les artiliciers ì essayer s'ils ne tireraient pas quelqu'avantage de cette poudre résineuse restée jusqu’ici inutile.

\section{Spathe.}

L'enveloppe de l'ópi on spathe est composion de phusients fenilles avec lesquelles on fail de bomes paillasses qui procurent un tris bon roucher. On se sirl aussi des feuilles de la

(1) Franģnis de Neufchattedu, ourvağe citt, p. 13! 


\section{( 216$)$}

plante : celles qui entourent l'épi sont flexibles, élastiques, elles sont préférables aux meilleures pailles et plus souples que les pailles de seigyle et d'avoine consacrées quelquefois à cette destination.

On ne doit choisir pour cette destination que les plus intérieures, parce que celles qui sont superficielles sont plus dures; elles servent alors pour la litière des animaux et principalement des pores.

J'ai pris des renseignnemens auprès d'une personne instruite qui habite depuis très longtemps la Bourgogne et qui m'a assuré que ces paillasses duraient huit à dix ans. On les vend maintenant fort avantageusement ì Strasbourg. Il y en a plusieurs dépôts à Paris. Dans l'été, on met les paillasses au dessus des matelas, pour être couché plus fraichement.

Chez les particulier's riches du Brésil, la couche se compose simplement d'un matelas de paille de maïs séparée en lanières étroites. Ces mêmes feuilles servent. aussi quelquefois à faire des corbeilles de différentes grandeurs et formes.

Willian Cobbett, dans son ouvrage sur le 
mais (1) dit qu'il a fait faire asec l'emsoluppre de l'rpi un paryue de papier sur lequel a ére imprimere la premiere fenille de son livre; ce piyper assez mince parait de bonne qualité, par le travail peut-ètre l'obtiendaraiton plus blanc.

\section{hirlle.}

Lat ralle sert, dans certains pays, pour allumer le feu ou chaufler le four; en Egyjte, pour les finhriques gui ont besoin de fen clair.

Sur les bords de l'Urénoque, les indigenes du pays qui nont pas de liége se servent des rachis de mais pour faire des bouchons (2).

\section{Son.}

Le son du grand mais jaune est forme de ¿randes plaques du péricange qui servent, en ltalic, à cmballex les objets précieux (5).

(1) Willian Colsbell, ouvage sur le mais (en auglais), in-12. Londust.

(?) De Humboldt, 7 o) ugre aux rémions equinoxiales, t. VII, p. 2y) I I1-8 .

(3) Monnegraphlic dess circules de la sumssi, pau lihates Serimge. Berme, 1818 , in-8", p. 182. 


\section{Farine:}

La farine de maïs se trouve souvent employée, en Allemagne, dans une recette pour empoisonner les taupes et les souris, qui a été publiée par M. Carl de Strasbourg, membre de la Société d'horticulture de Paris (1).

4. Un quart de livre de blé de Turquie;

Une once de vert-de-gris ;

Trois onces de chaux vive;

Douze écrevisses ;

Un quart de livre d'huile d'aspic.

Pilez et mêlez pour en faire une pàte en y joignant un peu d'eau de rivière, puis confectionnez des pilules de la grosseur d'une noisette, et mettez une de ces pilules sous chaque taupière ou dans les galeries des souris des champs.

(1) Annales de la Société d'horliculture, t. XI, p. 46 , 1828 . 


\section{EMPLOI LA MEDECINE.}

\section{Usuge du mais comme médicament.}

Nous venons d'étudier, dans un chapitre préecedent, less usages du maïs pour la nourriture des anmanx, et mous avons vu que ses principales parties, c'est it dire les tiges el les graius, leur convenaient beatcoup, ct que certains d'entr'enx en etaient très avides. Cet instinct, qui les porte i rechercher me plante qui les fait profiter à rue d'ocil, nous démontrerait dejà le parti que l'homme peut en tirer, sinous ne savious que, de tempss immémorial, il a été employé soit comme médicament, soit comme nourriture : nous allons done l'examiner sons cess deux rapports.

Comme médicament, les médecins du Mexiyue l'emploient depuis fort long-temps pour preparer, comme unus le finisons soment ave l'orge, unc hoisson douce et temipérante, convenaut dans un gyrand nombre de maladies a aģuës.

Cenx qui vivaien du temps d'Hernandez ( I)

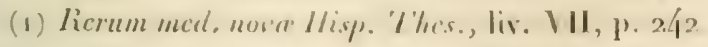
Romer, 1651, in-4́". 
ordonnaient souvent cette décoction comme émolliente et rafraichisşante à l'intérieur. De Laët (1) ne tarit pas sur ses nombreuses propriétés médicamenteuses, surtout sous forme de boisson nommée atole, qui se prépare à peu près commel'eau d'orge. L'expérience et le raisonnement firent voir aux Espagnols que le mais avait beaucoup de suc, qu'il était fort nourrissant, et qu'il était propre à guérir les maux de reins, les douleurs de la vessie, la gravelle et les rétentions d'urine. Ils remarquèquèrent sans doute qu'il n'y a vait presque pas d'Indiens qui fussent tourmentés de ces maux auxquels ils sont sujets eux-mêmes, et que cela venait de leur boisson qui est faite de maïs. D'ailleurs, les Indiens font de ce maïs une espèce d'emplàtre qui sert à guérir quantité d'autres maladies(2). Si nous nous rapprochons des temps où nous vivons, nous voyons ce grain encore usité contre d'autres maladies.

(1) Ourrage cité, liv. VIII, p. 321 .

(2) Histoire des Incas, traduite de l'espagnol de l'Inca Garcilasso de la Vega, par J. Bandouin, t. I, liv. II, ch. XXV. Amsterdam, 1704, in-12. 
Nous allons passer en revur les ressomrers quil peut fournio a la thrimaputique, et les (xemples de guérison obtemus avec hui. Sa décoction passe pour mucilagrineuse et apéritive : roici la maniere de la préparer si on se sert du grain entier.

On met le maïs bonillir dans l'eau, et apres une ébullition d'un quartd'heure on jette cett. première décoction. On concasse alors le grain, pour le cuire dans de nomvelle ean. Cette nouvelle décoction est édulcorée avec du sucre ou un sirop quelconque, et domée pour boisson ordinaire au malade.

M. le docteur Lespès, que nous aurons occasion de citer plusieurs fois dans ce chapitre, dit, 1. 44 (I), (que M. Ie docteur Double se sert souvent du maïs sous cette forme, et yu’il ohtient par ce moyen la guérison de maladies diverses. J'ai eu occasion de l'employer assez souvent dans ma pratique, et je

(1) Essai sur le maïs ou blè de T'urquie, considéré sous ses rapports hjogiénique "t médical, thèse soutemue, le 20 mai 1825, à la liacultí de médecine de Paris. 
suis satisfait des heureux résultats que j’ai obtenus.

Sa farine peut servir à faire des cataplasmes émolliens et maturatifs.

Charles-Étienne l'indique pour cet usage dans un passage de son Traité d'agriculture (r). En la comparant sous ce point de vue à ceux que l'on fait journellement avec la farine de graine de lin, on remarque qu'elle a sur cette dernière l'avantage de former des cataplasmes d'une odeur moins fade, s'aigrissant moins promptement, retenant une plus grande quantité d'humidité et surtout se desséchant moins, principalement sur les bords. On observe ce grave inconvénient dans les cataplasmes de farine de graine de lin qui, dans les hòpitaux, restent appliqués ordinairement pendant vingt-quatre heures, et au bout de ee temps sont trouvés sees et durs, par conséquent agissant sur des parties malades en sens inverse de l'effet que l'on se propose d'obtenir.

(1) L'Agriculture et Maison rustique de Charles Eliemne et Jcan Liébault, p. 5 ı . Paris, 1640. 


\section{(2.3)}

De nombrense's exprériences, fitiles sur les memes malades aree ces deux farines pulployeses en eataplasmes, me mellent à mente l'allimer ce que je viens d'avancer.

Quoique je puisse rapporter ici heancoup d'ohservations de son emploi, je me contentemi de décrire les deux eas les plus remaryuables qui se sont oflerts à moi.

I". Un de mes amis, le fils de M. de ${ }^{\star * *}$, portait depuis yuelyues jours un furonele assez cilendu et tris douloureux sur la partic postérieme ee interne de l'avant-bras droit; il rint me consulter et je hii fis appliquer, le prenier jour, un cataplasme de farine de mais. Le lendemain, tons les environs de la tument diatent mons el moins doulonreux. J'ordommai alors un camaplasme de larine de graine de lin. Le troisieme jour, l'aspect du furoucle était tout à fait changé, l. malade avait souffert beaucoup plus par la pression exercéc sur la circonférence do la tumeur par les bords du cataplasme desséché. Son aspect ctait violitre, et la pean environuante tenciue et tros sensible à la pression des «oiggts. Le quatrieme jomr, aprés l’applicalion, 


\section{(224)}

pendant vingt-quatre hemres, d'un eataplasme de farine de maïs, le furoncle était revenu à son état primitif, et la tumeur me paraissant assez molle je fis une onverture au sommet, par laquelle sortit du pus mêlé à une assez grrande quantité de tissu eellulaire gangrené. Les eataplasmes de maïs ont été continués jusqu'à parfaite guérison.

$2^{\circ}$. Madame Vannier, marchande de volailles près le marehé Saint-Germain, souffrait déjà, depuis plusieurs jours, d'un érysipèle phlegmoneux très étendu à la jambe droite, lors(qu'elle me fit demander le $1 \overline{3}$ août 1829. Après avoir interrugé la malade, et avoir examiné le membre attaqué d'érysipèle, j'ordonnai, avee une diète sévère, un repos absolu et l'application, plusieurs fois réitérée dans la journée, de eataplasmes de farine de maïs cuite dans de l'eau de guimauve. Le mieux ne tarda pàs à suivre ce traitement, et le 28 , malgré des imprudences réitérées et des affections morales très vives, j'ohtins une parfaite guérison. Depuis cette époque, j'ai eu occasion de l'employer sur moi-mème pour un furoncle à la main droite; chez M. Mayer, rue Sainte- 
Placide, pour une entorse; chez MI. Rossignol, rue d'Assas, $1^{\prime \prime} 5$, punr un panaris, ete., cte.

Celle farine de maïs, employée comme aliment sous forme de bouillie, est susceptible de produire une annélionation très sensible dans certaines maladies, et mème quelquefois la guérison dé quelyues affections particulières.

dinsi, quelques anteurs ont prétendu que le maïs était un bon préservatif contre le scorlutit.

M. Desbiey a remarqui qu'elle dimimuait les accès d'épilepsic, ainsi il dit (1) : L'introduction de la culture dı maïs fut l'époque d'une heureuse rérolution dans le tempérament de nos laboureurs. L'épilepsie était une des maladies les plus communes dans ce canton où la caudelée (ou cruchade), faite avec la farine de millet, était la pruncipale nourriture des habitans. Depuis que la farine de blé d'Espagne a remplacé celle du millet, pour

(1) Mćnoire sur la meill'ure manière de tirer parti des landes de fiordeaux, ele., qui a remporté, en 1776 , le prix proposé par l'Académie rnyale de Bordeaux, in-f", 1776. 


\section{$(226)$}

faire cette espèce de bonillie, le nombre des épileptiques a diminué sensiblement, au point même qu'ils y sont très rares aujourd'hui.

M. le docteur Lespès cite dans sa thèse (1) le fait suivant : M. Meyrac, pharmacien à Dax, dont le nom est connu dans les sciences, a remarqué que les paysans du Marancyn, partic des Landes où le maïs n'est pas encore admis, vienuent fréquemment chez lui acheter des remèdes contre l'épilepsie, tandis qu'il ne voit que très rarement pour cet objet ceux qui les avoisinent et qui s'en nourrissent. Enfin l'auteur dit tenir de la source la plus respectable qu'un jeune épileptique, demeurant à Paris, a vu, par l'usage du maïs que lui avait conseillé une personne qui connaissait le mémoire de Desbiey, les attaques de très fréquentes devenir extrèmement rares.

Nous engageons de nouveau les médecins à qui cet ouvrage passera sous les yeux à employer dans leur pratique, et surtout dans les hôpitaux, ce remède si innocent contre une maladie aussi cruelle.

(1) Thèse citée, p. 35 . 


\section{$(227)$}

Les samvagres or les naturels de l'Amérique, dans leurs chasses et lemrs Iongues comrses, pour aller combattre leurs ennemis, font usage d'une farine faite de blé d'Inde, et apres avoir vécu pendant des semanines et mène des mois entiers sans autre aliment, ils se trouvent non seulement vigoureux et pleins de santé; mais mème les blessures qu’ils ont recues se guérissent avee une faciliti merveilleuse (1).

M. le docteur Lespes a recueilli des observations de gastrites reconnues, d'entrites chroniques, même avec dysenterie, existant les unes et les autres depuis un temps quelquefois fort long et qui ne se sont calmées entic. rement ou nont disparu que lorsque le malade a été soumis à l’usage du maïs.

Je vais seulement rapporter la plus curieuse; pour les autres, je renvoie à la thèse (2).

M. Lessejs, consul général de France i Lisbonne, àgé de quarante-ciny à cinquantc ans, d'un lempérament nerveux, était depuis

(1) Dictionnaire de l'Industric, par une société de gens de lettres, t. $\mathbf{~}$, p $^{3}$ 338. Paris, $179^{5}$, in- $8^{\circ}$.

(2.) These, p. 4t. 
assez long-temps tourmenté par des souffrances vives vers l'abdomen. Outre l'inappétence, des digestions très pénibles, douloureuses, mème suivies d'évacuations promptes, de flatuosités, d'un gonflement de ventre, tel que la respiration en était souvent gênée, $M$. Lesseps était affecté d'hémorrhoïdes, il était périodiquement en proie aux crises de cette dernière maladie. Des écarts de régime, des affections morales vives, résultats de sa position, qui l'oblige souvent à prendre une part active à des affaires plus ou moins graves, exaspéraient frèquemment ses souffrances. En i 808, la maladie prit une forme aiguë; on réussit à la calmer, mais les symptômes chroniques persistaient. Il fut, m'a-t-il raconté, peu de temps après opéré de la fistule; je ne sais s'il se trompe, mais il me parait plus vraisemblable que l'on excisa chez lui quelque tumeur hémorrhoïdale. Quoi qu'il en soit, sous les autres rapports, son état ne devint pas meilleur, il empira même. Parmi les symptômes, l'état de ses excrémens l'effraya; il crut avoir la maladie noire. Douze à quinze années de cette existence pénible s'étaient écoulées, lorsqu'il 
réclana à cente occasion les couseils d'un jeune médecin porturais, qui élait arrivé depuis peu de France, oi il avait fait ses études médieales. Le: médecin voulut soumetro. M. Lesseps à un reggine sévère; il parla aussi de sangsues. Le malade s'y refusa. Il accordi plus de confiance à un docteur anglais. Celuici le traita ì la facon de ses compatriotes. Des purgations aussi violentes que prolongées nettoyèrent le canal intestinal ; car, dit le malade, il ne sortait que de la bile pure. Durant ce traitement, ses affaires l'anenirent ì Paris, où M. le docteur Bourdois lui prescrivit m régime très adoncissant. Le tapioka, le salep, l'arow-root, le sagou en firent tour à tour li base. Il le suivait. régulièrement depuis longtemps, n'ayant guère plus à s'en louer qu’à s'en plaindre, lorsque M. le docteur Legros l'engagea à essayer des préparations de maïs, et il assure que, dès le premier jour, il s'en trouva bien, car la digestion fut à peine laboricuse, et le besoin d'aller à la selle se fit. éprouver à une heure plus éloignée du repas qu'à l'ordinaire. L’appétit, nul depuis longtemps, devint très vif. Un pareil succès l'en- 


\section{( 230$)$}

couragea ; il continua, et depuis cette époque sa santé n'a cessé de s'améliorer. L'embonpoint et les forces revinrent rapidement. Il en fait depuis plus d'un an un usage journalier. Lorsque par une des causes indiquées plus haut, ou par quelqu'autre inconnue, il se sent indisposé, il fait dominer le maïs dans les alimens, et l'équilibre se rétablit.

M. Lespès, affecté d'une gastrite chronique qui redevint aiguë sous l'influence des chaleurs de 1827 , dit, dans son mémoire, n'avoir pris d'autre nourriture et avoir vu sous son influence sa santé se rétablir parfaitement et très promptement.

M. de Grégory dit qu'un potage excellent et nourrissant pour les malades est la bouillie de farine de maïs an bonillon de veau, à la façon d'une semoule.

Le maïs, comme je le démontrerai plus tard, est légèrement laxatif, il auggmente la quantité des urines et la sécrétion laiteuse On pourrait donc l'employer dans quelques cas de constipation, et lorsqu'on désirerait augmenter la quantité des urines ou la sécrétion du lait chez les femmes qui allaitent. 


\section{$251)$ \\ IU MAIS

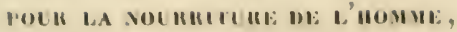

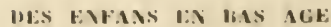 \\ ET DES FEMMIS UU ALLATENT (1).}

Telle est la question posée par l'Acadénie de médecine. Les raisonnemens enssent dé fort bons ici sans doute, mais ils ne sont plus que peu de chose auprés de l'expérience. Cette. idée est très vaie pour l'ohjet que nous traitons, car j'aurais pu croire conseiller d'employer le mais sous telle ou telle forme, si l'expérience ne fùt venue pour me prouver mon erreur. Aussi ai-je eu soin de n’éprargyner ancun sacrifice pour tacher, comme on l'a fait pour la pomme de terre, de multiplies les ressonrees que cette plante pent nous offir. Pendant plusieurs mois, jai fait des tentatives exuèmement nomireuses, changeant, modiliant saus resse pour obtenir des produits plus

(1) Tonte celte derniere partic de mon travail se trouve inséréc dans le premier lascicule du tome II les Memoires de l'Académic royale de médecine, publit i la lin de 1832. 


\section{( 252$)$}

parfaits. G'est appuyé sur plusieurs centaines d'essais que je vais donner ici le résultat de mon travail. J'indiquerai exactement le succès et la non-réussite.

Chacun des produits obtenus a d'ailleurs été soumis à la dégustation de plusieurs personnes de tout âge et de tout sexe. Les expériences médicales ont été très souvent répétées depuis le 15 janvier 1830 , époque où j’ai remis mon mémoire à l'Académie; je n'ai rien trouvé qui pût contredire mes premiers essais. Pour ne pas fatiguer le lecteur, je me bornerai à en consigner les résultats. Ce n'est qu'après avoir indiqué si ces alimens conviennent aux hommes, aux enfans en bas âge et aux femmes qui allaitent, que je parlerai d'une manière générale des effets de cette nourriture.

\section{TIGES.}

\section{Sucre.}

Un produit alimentaire important que l'on peut retirer des tiges de mais est le sucre, il paraît qu'il peut en domer des quantités assez considérables. 


\section{$(235)$}

Je demnerai dans son entien le travail de M. Pietet sur cette matière.

Culture du mats, en vae de la jabrication de sirop ou de sucre, à Lancy, près de Genére, par Cin. Pictet (1).

Le 25 avril 18 r , je semai en lignes espatcées de 28 ponces de la graine de gyrand maïs sur un espace de 5,80o pieds de superficie. La terre est de bome qualité, de moyenne consistance, plutòt forte que légère. Le champ n'avait point été fumé depuis trois ans, et avait donué du froment et de l'orge depuis lit fumure. Il avait été défoncé à la bèche pendant Ihiver. L'espace est de niveau et en plein soleil. Je semai trois fois plus épais dans la ligne 'qu'on est dans l'usage de semer, lorsqu'on veut recueillir la graine.

Le 26 mai, les plantes ayant 9 pouces de hauteur moyenne, je fis donner un sarclage à la main.

(1) Inséré dans les Anuules de l'agriculuere fran-

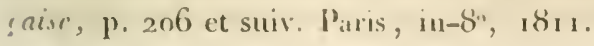




\section{( 234$)$}

Le 13 juin, je fis buter les plantes avec la petite charrue à un cheval, et à deux versoir's inobiles. Quelques plantes furent penchées par l'effet du versoir', on ne les redressa pas.

Le développement du maïs fut très rapide après l'opération du butage. Le 5 juillet, on commença à voir des fleurs mâles. Vers le 15, les houppes des épis se montrèrent sur quelques tiges.

Le 22 juillet, le développement de la plante étant complet, le maïs ayant environ 6 pieds de hauteur moyenne, les épis étant indiqués partout, et déjà un peu formés sur quelques pieds, et les étamines répandant abondamment leur poussière au moindre mouvement, je fis couper les tiges destinées à faire du sucre. J'avais remarqué que le bas de la tige était plus doux, lorsqu'on essayait de la mâcher, que les parties supérieures. Je fis donc couper très près de terre. Dans cette opération, j'eus égard à la convenance de conserver les belles plantes de maïs pour la graine, de les espacer suffisamment pour que les épis devinssent très gros, sans cependant laisser trop de distance, afin que la récolte du grain fiir 


\section{( 235$)$}

aussi abondante qu'elle pù itre, sur l'espace donné. Je tis enlever toutes le's feuilles et les fleurs mâles, parce que ces parties ne contiennent pas de sucre. Je fis hacher les cannes par morceaux de 5 a 4 pouces de long. Je pesai la totalité des cannes ainsi hachées : elles pesaient 117 livres, poids de mare. J'essayai de les faire triturer dans un bassin de roche, mais je vis qu'il yaurait beaucoup de travail et peu d'effets. Je les soumis à l'action d'une meule tournante que l'on emploie à peser sur les fruits.

Ce procédé fut très ellicace, et l'opération emploỵa toute la journée du 25. Le soir même, je fis placer la pulpe en masse sur un pressoir à vin, et jobtins 385 livres de jus. Ce jus était d'un goủt donceàtre assez semblable à l'eau de pomme que l'on donne à un malade.

Je fis déposer le jus pendant la muit dans un lieu bien frais, dans des vases de rendanges.

Le 24 , je distribuai le jus de mais en deux chandières de cuivre, dont l'une contenait 200 , et l'autre 176 livres; je soumis le liquide à l'ébullition à grand feu, 'n faisant ceumer avec soin. 
Je n'avais pas pu me procurer de la chaux éteinte par un long séjour à l'air; j'employai de la chaux éteinteà l'eau trois mois auparavant, priseau bord d'un creux où on l'avait fuscé pour bàtir. J'éprouvais le jus au sirop de violetteet au papier de teinture végétale, sans que ces réactifs m'indiquassent la présence d'un acide. Le papier bleu rougissait à peine tant qu'il était humide, mais il reprenait sa couleur en séchant. Ce goût indiquait cependant un acide très faible, très enveloppé, couvert par une autre saveur particulière au maïs , par un légुer goût vineux, peut-être dû au pressoir et aux vases employés; je me décidai à mettre la chaux peu à peu, sous forme pulvérulente, à la quantité prescrite par le professeur Burger, c'est à dire 14 grains par livre de jus.

Après deux heures d'ébullition, je commençai à mettre la chaux, et cette opération dura une demi-heure. L'écume se forma en plus grande abondance, tant que je répandis la chaux, et surtout dans les premiers momens, il parut en résulter une clarification sensible, et une conversion de la couleur verdàtre dı sucre en une teinte plus jaune. A mesure 


\section{( 257$)$}

que je répandais la chanx, je réprta is l'épreuve des riactifs sans observer ancune diflírener; et lorsque la quantité totale ent été répandue, le leger rougissement du papier bleu, en le trempant, avait lieu comme auparavant, et il reprenait de mème sa conleur en séchant. Le goùt de la liquenr me parut un peu modifié, mais moins agréable qu'avant l'opération ; ontre la saveur particulière an mais, la liqueur avait un goùt légèrement àcre et un arrièregoùt désagréable.

Je fis passer la liqueur dans un linge donble après sept heures et quart d'ébullition, c'est à dire lorsqu'elle fut réduite à la moitié de son volume. Il resta sur le linge, outre les partieules de chaux, heanconp de mucilage par petits flocons noiràtres. Le lendemain 25, après avoir laissé reposer la liqueur dans des vases de terre pendant dix-huit heures, je réunis le tout dans une chaudière, au fond de laquelle javais mis une livre de charbon de hètre, avec l'espérance d'enlever an liquide ce goùt àcre et étranger, qui se faisait remarquer au travers de sa donceur. Après deux heures el quart d'ubullition avee le charbon, je fis 


\section{( 238 )}

passer le liquide qui déposa encore beancoup de parties mucilagineuses. Il ne me parut pas que le charbon eût eu l'effet que j'en espérais. Le liquide plus concentré était devenu sensiblement plus doux; mais le goût étranger, quoiqu'un peu modifié, n'était guère moins désagréable. Pour éviter un accident qui arrive souvent aux sirops, savoir le brûlement, qui altère leur goût, je continuai l'évaporation au bain-Marie pendant toute la journée du 25. Dans le but d'èprouver si je pourrais concentrer le sirop à feu nu, sans qu'il prìt un goût de brûlé, je mis quelques livres du liquide dans un poèlon sur un feu très doux. Au bout de sept heures, il fut amené à un point de concentration convenable, c'est à dire qu'il filait en tombant de la cuiller, il était d'un brun doré bien transparent. Le goût étranger avait. presqu'entièrement disparu, et il était très doux.

Le 26, voyant quel'opération réussissaità feu nu, et trouvant celle du bain-Marie.trop lente, je soumis la masse du liquide à l'évaporation sur un feu doux dans de grandes bassines. J'en ai oltenu en tout 38 livres et 5 onces de marc. Ce à quoi ce sirop ressemble le plus, c'est 


\section{$(25 y)$}

an raisine, mais avee un arrière-gout moins acidule que celui-ci ne l'a ordinairement; il est anssi sensiblement plus doux. Comparé à la mélasse, il n'a ni antant de donceur, ni un gonit de sucre si franc. II a une très liggere amertume, et quelque cliose faiblement astringrent. Pour ne pas perdre l'occasion de l'expérience du bain-Marie, je mis ì part une livre dı sirop qui avait déjà été soum is à ce procédé, "t je contimuai l'opération. Le résultat a été sensiblement le même, c'est à dire que le gount du caramel s'y retrouve comme dans celui qui a été épaissi à feu nu.

Les palais délicats s'accommoderaient mal de ce sirop dans le thé on le café; mais il peut trouver son emploi dans plusieurs préparations ollicinales, telles que les crèmes, certaines sarices, des compotes et des gàteaux aux fruits.

\section{Comple des firais.}

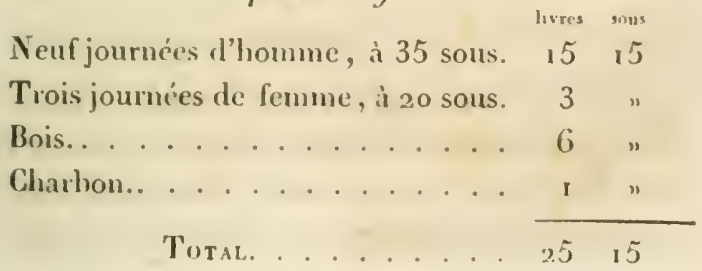




\section{( $2 / 40)$}

A ce compte, les frais sur l'arpent métrique de 94,840 pieds de surface s'éleveraient à 121 livres tournois, pour obtenir 6 I $_{2}$ livres poids de marc de sirop; lequel, d'après les expériences du docteur Burgos, doit rendre la moitié de son poids, c'est à dire 306 livres $\frac{1}{4}$ poids de mare de moscouade, laquelle reviendrait, par conséquent, à 23 sous la livre de marc ( 1 ).

Si l'on considère que, dans le procédé que j’ai employé, la récolte du grain de mais demeure à peu près aussi abondante que si la plante n'eùt été cultivée qu'en vuc du grain; si l'on réfléchit que la culture du maïs occupe déjà une grande partie du sol français, et qu'elle est en Italie la culture principale, on sentira que cet objet est d'une très grande importance et qn'on ne saurait trop en encourager les effets.

Nota. Il faut avoir soin d'enlever soigneu-

(I) Il ne faut pas oublier que l'arpent rendrait le double s'il était cultivé uniquement pour le sucre, c'est à dire 306 kilogr. de moscouade. Il eu rend 222 par la culture de la betterave. 


$$
(2,1)
$$

sement les ferulles qui enveloppent les tiges "l celles dans lespuelles les jeunes épis sont renfermes, elles ne eontienuent point de sucre el ont un gonit do vert melé d'un pen d'anertume.

Suivant de Humboldt, an Mlexique et dans les contries voisines, on retire avec avantage du sucre des tiges de maïs.

M. Ie docten Roulin, dans un mémoire hu le 20 juin à l'Acadćmic des sciences, fait remarquer, en terminant, que la température influe beaucoup sur la proportion des principes immédiats fui entrent dans la composition du maïs; c'est ce dont il a eu occasion de s'assurer pour la tige de cette gramince. Dans les pays froids, comme Bagota, la tige du maïs n'a (qu'une saveur très insipide, mais à Maraquita, où la chaleur est très forte, cette même lige est sucrée et fournit en ahondance un sirop tres agréable.

Pamentier, qui a fait de nombreux essais pour retirer du sucre el de l'amidon du maïs, doute que l'on puisse jamais les compter comme des ressources.

On lit ce qui suit dans la note de la page i 54 : 


\section{( 242$)$}

"Si l'oui n'est dans l'intention de n'extraire le sirop de maïs que de la tige de cette plante destinée an fourrage, on peut en effet laisser entrevoir au cultivateur l'espérance d'un bénéfice réel, puisque, sans préjudicier bien sensiblement à la nourriture de ses bestiaux, il peut se proeurer du suere pour son usage; mais je pense qu’il y aurait de la folie à vouloir employer, pour l'obtenir, des tiges de maïs semé et cultivé pour grain, et se priver par là d'un aliment si nécessaire aux habitans de certains pays, et dont l'excédant est en état de suppléer le froment dans les endroits où ce dernier grain viendrait à manquer. "

N'ayant pu me procurer à Paris une grande quantité de tigges fraiches, je n’ai pas fait de suere de maïs; mais je crois que si un propriétaire se livrait en grand à cette fabrication, on obtiendrait, après quelques tâtonnemens, un produit qu'on pourrait livrer à bon compte, et qui entrerait souvent dans la préparation des alimens des hommes, des femmes qui allaitent et des enfans eı bas âge. 


\section{$2 / 15)$ \\ Jiges confites au sucre.}

On confit, dans I Amérique septentrionale, la hampe qui se trouve dans les oflicines ( 1 ). Si l'on voulait composer cette frimdise, on aurait la ressource de confire dans le sucre mème du maïs, ce qui ue lenr enleverait rien de leur parfum particulier.

\section{EPIS NON MURS.}

Les petits ćpis dlu maïs, que l'on arrache m mème temps que les fenilles inférieures, peuvent ètre vitilisés pour la nourriture de différentes manières.

\section{Mais frit.}

Dans quelques pays, on domne à cette préparation le nom de heignets de maïs. Après avoir dépouillé les jeunes épis de leurs feuilles et de la bonrre qui les enveloppe, on les fend

(1) Willemet, Phorographie ency-clopédique ou Finre cconomique, t. III, p. 1623. Paris, 1808. 


\section{( 244$)$}

en deux ou trois portions, suivant leur longueur et on les fait frire avec la pâte comme les artichauts.

D'après les renseignemens que j'ái pris en Bourgogne, ce mets délicat y est très usité lorsque le maïs est jeune ; il convient beaucoup aux hommes, aux femmes qui allaitent, et si on le donne aux enfans en bas âge, ce ne doit être qu'en petite quantité.

\section{Maïs grillé.}

Chez les Américains surtout, on fait griller les jeunes épis devant le feu et on les mange tout chauds.

Parmentier blàme beaucoup cette méthode; il dit, page 21 5 : "Il existe des contrées en Amérique où les habitans consomment une si grande quantité de maïs pendant que les épis sont encore tendres, qu'ils diminuent sensiblement et détruisent même leur récolte. "

A supposer que ces peuples aient une si grande avidité pour un aliment si peu nutritif, on ne peut toujours pas en faire une nourriture habituelle, et il ne conviendrait qu'aux hommes qui ont les estomaes robustes. 


\section{$(2 / 5)$}

Maus, farine froide.

D'apres Parmentier (1), it y a une atutre manière de so servir du maĩs tendre et en lait, en égrenant les épis, en les grillant dans un pot de terre, ou une marmite sur le feu; il en risulte une farine jaune, qui a un petit goùt de café, c'est ce qu'on nomme, à la.Louisiane, farine froide. Pour la manger, on ne fait que la détremper à froid avec un peu d'eau, ce qui forme une bouillie fort agréable et fort saine.

Je crois que cette bouillie conviendrail peu aux hommes, paree qu'elle soutiendrait moins leurs forces qu'une autre préparation de maïs, mais elle pourrait bien réussir aux femmes qui allaitent et aux enfans en bas àge. Les organes digestifs de ces ètres si faibles ont besoin d'une nourriture qui les excite pen et leur facilite la transition de la nourriture que leur offre le sein de leur mere avec colle plus solide qu’ils doivent prendre un jour.

(1) Ouvrage citc, p. 2.16. 


\section{$(246)$ \\ Mais confit au vinaigre.}

Les jeunes épis de blé de Turquie peuvent être confits au vinaigrecomme les cornichons, aussi leur donne-t-on le nom de cornichons de maïs.

On doit se servir de vinaigre blanc; on fait fondre une livre de sel dans une pinte d'eau chaude; on tire l'infusion à clair, et on y ajoute une pinte de vinaiggre, on remplit le vase de jeunes épis qui se trouvent confits et bons à manger un mois après. Pour conserver leur blancheur, on met par dessus un lit d'estragon et de perce-pierre qui leur donne en même temps un goùt plus agréable. Quelques personnes y ajoutent aussi d'autres épices.

Les cornichons de maïs se préparent encore quelquefois en les mettant dans l'eau bouillante, où on les laisse cuire jusqu'à ce qu'ils fléchissent sous le doiggt et restent encore croquans; on les jette alors dans le vinaigre. Ces cornichons ont, sur ceux faits avec des concombres, l'avantage d'être moins charnus et plus compactes, par conséquent plus pro- 


$$
\text { ( } 2,17 \text { ) }
$$

fures anx voyages de leng; contrs; anssi en expedic-t-on beancoup ponr les colonies. Ils comviemuent beancoup) anx hommes qui s'en serrent comme assaisomement, mais les lemmes qui allaitent et les enlans en lats aige ne doivent pas en user.

\section{Mass conful au sucre.}

Je crois, sans pouvoir toutelois l'allirmer, qu'il serait possible de confire an sucre les jeunes épis de maïs.

Un confisem à qui j'en ai parté est aussi de cet avis el se propose de firire cen essai all printemps prochain, s'il peut s'en procurer une certaine quantité.

\section{Maıs blé doux.}

Si, par suice d'un retard dans les semailles on par lat mauraise saison, on ne croyait pas voir mùrir ce grain, on pourait le préparer comme les Indiens (1). Quand le błé, qui ust

(1) Frangois de Neutchitcau, ourrage cité, 1. 29.

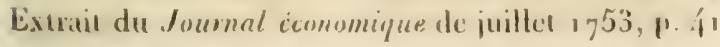




\section{$(248)$}

dans l'épi, est plein et encore vert, il at un goût fort agréable : ils en coupent alors une certaine quantité qu'ils font bouillir et sécher. Dans cet état, ils le mettent dans des sacs pour s'en servir au besoin; 'et ils le font encore bien cuire dans son entier ou broyer grossièrement lorsqu'ils veulent le manger soit seul, soit avec du poisson, de la venaison, du castor ou quelqu'autre viande, ce qu'ils regardent comme un mets fort délicat.

\section{Maïs bouilli.}

Les gुrains de maïs non mûrs sont, comme on sait, doux et sucrés. Les Indiens l'égrènent dans cet état, le cuisent et l'accommodent à l'instar des petits pois auxquels ils le préfèrent. Ce qui fait les délices des Mineros (Brésil), c'est le plat de cangica, nom qu'ils donnent au maïs dépouillé de ses enveloppes et simplement cuit dans l'eau. Rien n'égale, dit M. Saint-Hilaire, la fadeur d'un tel mets, et pourtant on s'étonne de ce que l'étranger a assez mauvais goût pour y joindre du sucre.

Parmentier a essayé de différentes manières 


\section{( 249$)$}

à employer le matis soms cette forme, mais il dit ne pas en avoir obtem 1 m bon eflet, parce quil est diflicile à égrener el ne erére pats licilement dans l'ean.

J: de Laët et Amoreux disent, au contraire, que cette préparation ne le cede pas aux petits pois.

Lorsque le grain est 1 peu plus avancé rers sal milturité, qu'il est encore vert, mais it l'élat farineux, les Chiliens bo broient entre demx pierres comme du cacao, et ils en font unc pàte qu’ils mettent avec du beurre el du sucre, ils le font ensuite bouillir dans l'eau (1).

\section{Muis orgeat.}

Parmi les Européens, les Espagnols cueillent le maïs Iorsqüil cst encore tendre et en lait, ils le broient eu y ajoutant un peu d'ean; de cette maniere ils en font une espece d'orgeat au lait qu'ils nomment mandorle, dans lequel ils.mettent du suere, de l'ambre el

(1) Molina, Mistoire nature llc du Chili, laduite de litalien par Gruvel. 1789. 


\section{( 250$)$}

d'autres aromates. Ils regardent cette boisson comme pectorale et la mêlent quelquefois au chocolat ( 1 ).

Cette boisson, qui plait beaucoup aux hommes, conviendrait également aux femmes qui allaitent et aux enfans en bas âģe.

\section{Mais cru.}

Il est arrivé en Piémont que des' militaires, et notamment des Autrichiens, ont arraché les épis du maïs quarantain encore vert et les ont mangés ainsi crus (2). A Candie, on l'emploie aussi assez souvent; mais sous cette forme le maïs ne convient qu'aux estomacs vigoureux des hommes.

\section{GRAINS DE MAIS.}

Boisson.

Chicha. - Les Américains prépareırt avec te maïs une boisson favorite qui les cnivre et

(1) Ianasti, ouvage cité (en italien), p. 100.

(3) Instruction pratique du P. Buniva, ouvage cité 


\section{( 25 I )}

quils appellent chichn, laquelle, apres une fermentation sullisante, acpuiert une aciditi agréable. Ils attribuent à cette boisson quetques verus particulicres. Chez les Péruviens on la nonme asun ou Eara, elle est blanche ou roure suivant la couleur du maïs dont on l'a faite.

Acosta (1) indique ainsi- sa préparation : "Il se fait en facon de cervoise, mettant tremper premierement te grain de mais jusqu'i ce qu'il se crève; peu après ils te cuisent d'une telle façon et devient si fort, qu'il en faut peu pour abattre son homme. Au P'érou, ce breuvage est défendu par les lois, à caus: des grands inconvénieus qui en proviennent, enivrant les hommes. Mais cette loi est mal observéc d'autant qu'ils ne laissent point d'en user et passent les nuits et les jours cutiers à en boire en dansant et ballant."

D'autres auteurs disent qu'ils font ròtir le gyin jusqu'ì ce cu’il devienme en charbon, et après l'avoir pilé le jettent dins de grandes

(1) Histoire naturelle it morale des Indes, Laduite.

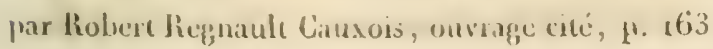


chaudières d'eau, où ils le font bouillir ; cette eau noire et dégoûtante fait leur délice. J'ai fait préparer du chicha sous cette dernière forme, et quoique cettc liqueur se rapprochàt beaucoup du café par sa couleur, que le sucre n'y füt pas épargné; je puis assurer que cette boisson est insupportable au goût. Des personnes présentes et qui voulurent bien en goûter furent du même avis.

\section{Petite bière (I).}

Lorsqu'il s'agit de célébrer une fête ou un mariage, les Indiens font infuser 20 ou 3o boisseaux de maïs dans une auge pleine d'eau, jusqu'à ce qu'elle soit imprégnée du grain et qu'elle commence à s'aigrir : alors quelques vieilles femmes, qui n'ont guère autre chose à faire, mâchent des grains de maïs, qu'elles mettent dans des calebasses, et quand elles croient en avoir assez, elles versent ce

(1) Description de l'isthme de l'Amérique, par M. Waser, insérée dans le Nouveau Yoyage autour du Monde, par G. Dampierre, t. IV, p. 256 et suiv. 


\section{$(253)$}

milange de mais en de salive dans l'anyze, apres en avoir retire le gyain quion y arait fait infuser. Cette esprece de bouillie sert de levain, et fait preadre une ligrere fermentation ì toute la liqueur. Quand elle ne fermente plus, on la tire it clair dans une antre ange; elle a le roùt de la petite biere yui est aigre, et arec cela elle entète beaucoup. Its en boirent à longs traits, et en sont fort avides, quoiqu'elle leur donne quantité de rapports; c'est leur boisson délicieuse les jours de gala.

\section{Posole.}

Selon Guillaume Dampierre (1), il paraic que la baie de Campèche et les autres paỵs roisins sont très fertiles en maï; les peuples 'qui les habitent font arec ce grain une boisson aigrelette, en mettunt sa farine, sous forme de pàte, dans une jarre et l'y laissant jusqu'i ce qu'elle soit aigrie, et dès quïls ont soif, ils

(1) Foyage aurour du Monde. Ionen, 1715, $111-12$. 


\section{( 254 )}

en mêleṇt un peu dans une calebasse remplie d'eau, ce qui lui donne encore mn gुoût plus piquant et plus agréable.

Ils passent ensuite le tout par une autre calebasse plus grande, percée de trous, pour en séparer l'écorce du mais ; ils boivent cette liqueur à laquelle ils mêlent quelquefois dı miel, elle leur parait aussi bonne qu'à nous un verre de vin.

Entreprennent-ils un voyage de deux ou trois jours, ils emportent avec eux pour toute provision un peu de pâte de maïs aigric, enveloppée dans une feuille de plantain, et une calebasse à leur ceinture pour préparer leur boisson qui leur tient lieu de nourriture jusqu'au retour chez eux. Elle est connue sous le nom de posole.

\section{Atextili.}

A Guatimala, on prépare une boisson avec parties égales de grains de cacao el de grains de maïs; on broic bien le tout, on fait bouillir jusqu'à ce qu’il se forme de l'écume, on transvase alor's, et on boit. 


\section{$(255)$}

Lorspuion rent une lowisson phus forte, on $\checkmark$ ajoute du poive long or de la vanille (1).

\section{T'sne.}

Cette boisson, faite dans le même pays, a beancoup d'analogic avee la précédente. On prend parties igrales de grains de cacao rôtis et de grains de maïs ròtis; on broic le tout, on fait cuire avec un peu de maïs cru, qui a dì bonillir quelque temps auparavant, et on hoit.

J'ai préparé cette boisson sans sucre, elle ist peu agriable el a un goùt amer assez prononcé, que lui communique le cacas.

Si l'on y ajoute du sucre, cer gout amer so perd et la boisson devient plus aggréalble. de crois qu'il faudrait y être habituc.

\section{Café.}

Dans quelques pays, en Riussir surtout, on

(1) De Laer indique trés au lon préparations 


\section{( 256 )}

prépare sonvent une boisson qui se prend après le repas, et qui remplace le café que l'on prend en France. Cette boisson consiste en une décoction d'orge légèrement grillée.

J'ai voulı voir si le maïs pourrait donner quelque produit qui approchât de cette préparation ; une décoction de grains légèrement rôtis, sans sucre, avait un goût particulier peu agréable.

Avec du sucre on pouvait la boire, mais le résultat n'était pas toujours à vanter.

Une autre décoction de parties égales de poudre de grains rôtis et de poudre de café donne une liqueur noirâtre assez bonne; mais ce serait gâter une bonne chose pour une mauvaisè, il faut s'en tenir à notre café. Depuis quelques années, on vend, à Paris, un café en poudre dit de la Trinité, dont j’ignnore la composition, mais qui a beaucoup d'analogie avec eelui que l'on peut préparer avec le maïs.

En janvier 1833 , j'ai cu occasion de trouver à acheter différentes préparations de maïs, et entr'antres du café tout gyrillé. Son emploi 


\section{( 257$)$}

m'a parn plus agreablibe que dians mes prentiers essais ( 1 ).

\section{Bière.}

II n'est pas douteux que l'on ne puisse faire de la biere avec le maïs; les Anglais et les Aurichiens en préparent, dit-on, heancoup arre cette semence. Le grain qui parait préfúmble pour cette opération est le maïs bleu.

II y a trois manières principales de la préparex :

$1^{\circ}$. Avec le pain de mais ; $2^{\circ}$ en enterrant le grain pour le faire germer; $5^{\circ}$ par la méthode ordinaire.

La manière de fahriquer la bière avec le pain consiste à le couper par morceaux de la frosseur du poignet, ensuite à l'éeraser et à s'en servir comme de drèche pour fabriquer la biere avec el sans houblon.

Il y a une maniere particuliere pour faire de bonne drêche arec le mais mème. On a remarqué qu'avant d'être en drêche, le grain

(1) Chez M. Porcheron, marchand de pâtes, passage Choiseul, $11^{\circ} 14$, à Paris. 


\section{$(258)$}

devait germer beanconp par les deux bonts, c'est à dire par la racine ef le tuyau, opération diflicile par les procédés ordinaires, parce qu'il y a à craindre la moisissure si le las est trop épais et qu'il reste long-temps ainsi; d'un autre côté, si on remue le tas, les grains qui ont commencé à pousser cesseront de croître. Pour remédier à ces inconvéniens, on a essayé, avec succès, la méthode suivante (1): creusez de 2 ou 3 ponces la surface de la terre dans un jardin ou dans un champ, et jetez la moitié de cette terre d'un còté et la moilié de l'autre; ensuite mettez sur ce terrain le blé que vous destinez à fairè de la drêche, et recouvrez-le avec cette terre; laissez-le en cet état jusqu'à ce que vous voyiez tout ce contour de terre comme un champ vert couvert de fenilles de blé, ce qui arrivera au dixième ou quinzième jour, suivant la saison; ensuite levez-le de terre, nettoyez-le et le faites sécher. Les racines seront

(1) Franç. de Neufchâteau, ouvrage cité, p. 31 et 32. Extrait du Journal économique du mois de juillet 1753, p. $43,44,45$. 


\section{( 259$)$}

tellement combarrassées les unesdaus lesautres, que l'on pouratit en enterer de grands morсеанx.

Pour le bien nettoyer, il fiut le laver et to faire sécher promptement sur un four ou an soleil; de cette maniere, tout le grain qui est bon se développera , deviendra fondant, moelleux, fort doux, et la biere qu'on en fera sera saine et agréable, d'une bellecouleurbrune.

Gependant la biere faite aree le pain, conme nous l'arous dit ci-dlessus, est anssi colorée, aussi saine et aussi agréable, elle se garde plus Ionơg-temps. Parmentier (1) a fait préparer de la bière de maïs par le procédé ordinaire. M. Longehamps, qu'il avait chargé de ce travail, a observé que le maïs consommait plus d'eau au trempoir, qu'il paraissait moins se renfler et se ramollir que l'orge ; qu'il fallait un pen plus de temps pour operer la germination et la dessiccation; qu'enfin la saveur dait un peu moins sucréc. An reste, la bière qu'il a obtenue ćtait léģère, pen colorée, quoiqu'aussi houblonnee, et son amertume dimi-

(1) Ourrage citi, p. 2.12 . 
nuait à la longue sans rien perdre de son agrément.

Frappé de l'idée que cette bière pourrait avoir peut-être quelque propriété particulière, j’ai aussi voulu essayer à en faire faire. A cet effet, et pour ne pas m'engager dans de trop fortes dépenses, je donnais à M. Fischer, brasseur au Gros-Caillou, vis à vis l'Hôpital militaire, 8 o livres de grains de maïs de l'année provenant de la récolte de $M$. le baron Ternaux à Saint-Ouen, e'était la quantité la plus minime sur laquelle on pût raisonnablement opérer; mais malheureusement les froids très vifs et trop long-temps soutenus de l'hiver de 1829 ont empêché le grain de germer convenablement, quoiqu'il eût été placé dans une chambre toujours chauffée et à côté du feu. Peut-être que du grain de la récolte précédente ( 1828 ) eût été préférable, j'avais agi dans une opinion contraire. J'invite les personnes qui cultivent le maïs en grand à répéter cette expérience, elle pourrait peut-être donner des résultats avantageux et ouvrir un nowveau débouché à leurs produits.

Cette bière conviendrait, je pense, aux 


\section{$(26 i)$}

hommes, el en perite quantite aux femmes qui allaitent el aux enlans (no bas ige.

Dans lo Foyage des fières Landers en Afirque, il est question d'une bière excellente faite avee lemilis.

\section{Eran de vie et vimaigre.}

Quoique Kalm nous assure qu'en Amerique on en lire une bonne eau de vie, et que nous sachions qu'on puisse en faire du vinaigre, il est douteux qu'en France on tente de lirer ces deux produits du maïs, puisque nous possidons du vin en quantité qui peut nous donner ces deux liquides à bon compte.

\section{MAIS EN GRAINS ENTIERS.}

\section{Alinent.}

Un respectable missionnaire, 11. l'abbé T'erray, supérieur du séminaire d'Issy, qui a pendant trente annces pareom'u le Camada, 


\section{$(262) \cdot$}

a communiqué à M. Bossangुe père la note suivante sur le maïs : elle a été insérée dan: les journaux de i 829 .

Les créoles qui transportent en canot les marchandises du Bas-Canada dans le HautCanada, et qui les portent de temps en temps sur leur dos par ballots de 200 livres, m'ont dit que, de toutes les nonrritures, celle qui les sontient le mieux, c'est le maïs mondé, euit dans l'eau et mangé gyrain à gyrain ; qu'il leur arrivait souvent de n'en manger qu'une poignée par jour, et que cela leur suffisait même dans leurs plus forts travaux. Quelques uns d'entr'eux m'ont assuré que, lorsqu'ils étaient dans les bois sans rien faire, une douzaine de grains par jour suffisaient pour les sontenir, et des sauvages m'ont cité des hommes, des femmes et des enfans qui, avec quatre ou einq grains par jour, avaient bravé la faim pendant plusieurs mois consécutifs.

Maìs róti.

Les paysans du midi font torréfier les prrains 


\section{( 26 ij )}

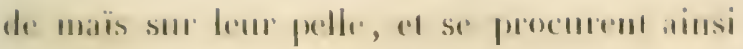

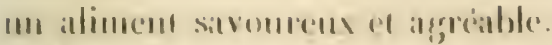

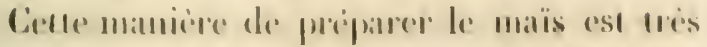

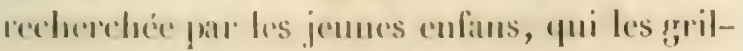

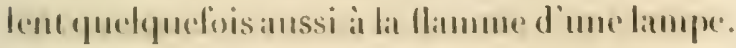

Cetle preparatson fort simple domne mo parfun particulicr aแ maiss.

Mas e'll grains cassés.

Voir, à la lin de la note communiquier par le doctenr Abel Hibon, l'usagre guién font les moirs el mene les colons de l'Ile-Bourbon.

Harine de la fiusée ou papeton.

Le papeeton a anssi recule lam de rafle, de prenot (en Piémont); les Bongynignons et les Comtois l'appellent fuseru, panouille, riberin, z̧uilledou. Dans le Haut-Languedoc et daus le Bearn, il est commu sous le nom de churbon blanc; ailleurs, conyurril ; dans le département de l'Homalt ínlos, et dans celui de Lot-et-Ganomue pelou.

En temps de diselle, on ponrail faire seches 


\section{( 261$)$}

cette fuséc, la faire moudre dans un moulin à blé; on en retirerait une farine dont on peut faire du pain et dans lequel on mettrait de la farine de froment ordinaire, plus ou moins, suivant que l'on voudrait que le pain fût léger. Tel est l'avis que donnent quelques auteur's, et entr'autres Lelieur ( 1 ).

Cette farine, mêlée avec de l'eau, peut aussi se donner aux vaches pour boisson, elle leur procure un lait abondant.

Elles refusent de la manger lorsque cette fusée est sèche; mais elles la dévorent avec avidité si elle est fraîche.

\section{FARINE DE MAIS.}

\section{Boisson.}

Chocolatl. Les Mexicains préparaient une boisson dans laquelle un peu de farine de mais, de la vanille (tlixochitl) et le fruit d'une es-

(1) Ourrage cité, p. 354, in-12. 
prece de piment (méraxochiel) étaicnt métés alu cacao (cacahuall) (1).

\section{Alimens. - Biscotes.}

Arec la farine de maïs, comme arec la fitrine de froment, on peut laire des biscotes, ‘ui l'emportent de beaucoup en gonit sur celles faites avec cette dernière farine.

L'Académic a pud'ailleurs enjuger par celles que je lıi ai soumises; elles avaicnt été faitẹ, le 2 décembre 1829 , par M. Bessa, fabrican de biscotes, rue de la Reỵie, $1^{\circ}$ 17. Ces petits bisenits se mangent secs on dans le thó.

Ils conviennent trés bien anx hommes, aux femmes qui allaiteut, surtout aux enfuns en bas àge ; ils aiment beancoup en avoir lor'squ'ils font desdents. Ce corps, qui s'imollit par ta salive, est bien meilleur pour cet usage, que les hochets en os ou en ivoire, ete.

(1) Humboldt, Essai politique sur la NousellcEspagne, 1. 11I, P. 2.4. 
Voici la préparation qu'indique Parmentier, page 250 :

On prend une certaine quantité de farine dece grain convenablement noulu; on y ajoute un peu de levain, qu'on délaie dans l'eau tiède, on en forme une pàte d'une consistance plus molle que celle qui est destinće au biscuit ordinaire. On en détache ensuite des morceaux pesant chacun 3 quarterons, qu'on aplati de manière à ne leur donner que 24 pouces de circonférence et 15 à 16 liggnes d'épaisseur. Quand la pâte est façonnée, on la distribue sur des tablettes, et peu de temps après on la met au four, en la piquant avec un fer armé de plusieurs dents, pour empècher le boursouflement et favoriser l'évaporation de tous les points. Il faut la laisser dans un four doux pendant deux heures au moins, paree que cette cuisson demande à ètre poussée très loin.

Il convient de placer le biscuit, au sortir du four, dans un lieu chaud, pour'qu'il puisseserefroidir insensiblement et perdre l'humidité quu s'en exhale perpéurellement, tant que la chalem 


\section{$(267)$}

subsiste. Il est done essemted de no le renfermex que cing on six jours apres sal fibrications.

Parmentier lit fare qualre bisenits de mats pur et qualte de mais et de froment par partices eigrales, yui furent emroyés par M. LafondLaddebat, le 25 arril 1785 , a M. Francois de Noufchiteau, alors procurem général au Lip-Frangais, qui les recut le 2.5 juin r785, apres 60 jomrs de laversce.

Les boiles qui les contenaient furent ouverkes en présence de beancoup d'hommes instruits, el on trouva gue ceux de maïs pur étaicnt assez bien conservés, compactes, d'unc sarcur aigre el d'un goùt amer. Macérćs dans l'eau, la saverur aigre parut moins considénable. mais le degré d'ancrtume a été plus fort.

Les biscuits de mals mélangés avee du froment, hien conservés, plus ligrers, n’ayant point la saveur aigre, mais développant leur amertume quolefue temps aprés la mastication, an frocit de yuelques personnes, eetle sensation n'ayant pas éte génćrale, denx de chanue faconfurent remoyes à Bordeau, mais ils furent allatues des insectes durant la marossece et l'on ne perut pas hien jurger de lemécall de con- 


\section{( 268 )}

servation. Cet accident parut avoir été causé plutôt par le défaut de soins que par la qualité du biscuit. Cette expérience si importante, dit M. François de Neufchåteau (1), n'a pas eu d'autre suite, parce que les esprits étaient occupés de toute autre chose, et qu'aux approches de la révolution la politique absorbait tout.

Il eût été à désirer que l'on reprît l'expérience, et qu'on s'assuràt des moyens d'empêcher l'aigreur et l'amertume du biscuit de maïs, surtout en essayant de mélanger de lá farine avec la parmentière ou pomme de terre.

Cédant aux vœux de ce savant, j’ai voulu tâcher d'obtenir un produit ntile; j’ai done fait confectionner dix biscuits de chacun des mélanges suivans :

$\mathbf{1}^{\circ}$. Parties éģales de maïs et de pommes de terre;

$2^{\circ}$. Parties égales de froment et de maïs;

$3^{\circ}$. Un tiers maïs, un tiers froment, In lier's pommes de terre;

$4^{\circ}$. Un tiers maïs, un tier's froment, un iiers seigle ;

(1) Oıvrage cité, p. 153. 


\section{$(269)$}

50. Partice égale de farine de mitis préalablement cuite daus de l'ean eomme la polentu et froment :

En tont, cinfuante biscnits.

J'arais demandé aussi à M. Bessa, qui les a fabriques, dix biscuits en farine de mais pur, mais ils ont éti oubliés, et le terme du concours n'a pas permis de les faire.

J'avais gardé six de chacun de ces biscuits, yne l'Acadénie devait mettre à la disposition du Ministre de la Marine, a fin quel'on pût ainsi apprécier leur qualité et leur deggré de conservation. Les érénemens subits de notre gloriense rérolution ont enpêché de prendre une décision à cet égard. En janvier 1832 , e'est à dire lorsquilis avaient déjà deux ans de fabriation, j’ai expédié à l'lle-Bourbon presque tout ce qui me restait; jiatlends avec impalience l'envoi du procès-verbal qui sera dressé, pour juger de leur état à l'époque de leur arrivée et de la honne ou mauraise qualité de cenx qui me seront renvoyés (i).

Ceux que j'ai encore sont tonjours très

(1) Jignore la cause du retard que j'éprouve, car 


\section{( 270$)$}

bons, ils ont cependant trois ans de fabrication. Tous sont secs sans ètre fendus, sonores, très dur's; ils se cassent net et trempent facilement dans l'eau sans s'émietter. J'ai répété quelques essais, ils font de très bons potages, et y sont préférables au pain.

Des persomnes qui ont parcouru l'intérieur du Brésil mont assuré que ces biscuits seraient d'un gyrand secours aux voyageurs.

Il serait possible de rendre ces biscuits plus uutritifs en y mélangeant une eertaine quanlité de gólatine.

\section{Boulettes de mais.}

Les Indiens écrasent ce grain et en font des boulettes qui leur tiennent lieu de pain. II. Lelieur (I) a fait usage de ces boulettes à la mer, et il conseille anx personnes qui veulent s'embarquer de se munir de farine de maïs torréfićc. L'usage en est facile et simple;

sept mois seulement sont nécessaires pour avoir la réponse, et quatorze se sont déjâ ícoulés.

(1) Ourrage cité, in-12. 1S27. 


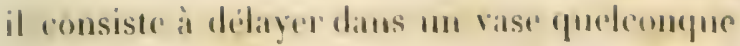
de la fariue de mails aver de l'ean ed du sel, d'en faire une pate lerme avee laquelle on forme des boulettes de la grosseur d'un ceuf de poule, on les jette dans la marmite de l'iquipage une heure arant te repas. Les persomnes qui ne sont pas accoutumées à la mer préféreront ees houlettes au biscuit; elles troureront, dans cette nourriture de facile digestion (th dans ses elfets adoucissans, un grand sonlagement au malaise qu'éprouve notre physi‘que sur un élément que nous ne pouvons commencer à habiter saus souffrir plus ou moins.

J'ai jeti des bonlettes, ainsi faites, dans le pot an feu, et an bout d'une heure elles ont ètéretirées; elles étaient plus volumineuses, lenr goùt était assez bon; un enfant de trois ans, qui se trouvait là, en a mangé une entière avec plaisir.

Chocolat oic la farine de mas remplice la fécule.

Sachantepuis longr-tempsune, dinus les ehocolats ordinaires, on faisait cutrer de la fécule, 


\section{( 272$)$}

et pensant que la farime de mais pourrait bien la remplacer et donner un chocolat très digestif et très nourrissant, j’ai fait faire plusieurs livres de chocolat où la farine de maïs est entrée dans la proportion d'un quart par livre, comme on le voit ei-dessous.

Cacao. . . . . . . 12 onces.

Cassonade. ....... 12

Farine de mais.. . . . . . 8

Son aspect est celui du chocolat ordinaire; il est dur, sa cassure est nette, et l'on voit briller quelques petites paillettes luisantes, que j'attribue au son duquel la farine n'aurá pas été entièrement purgée.

Son odeur et son goût sont agréables, et ne présentent rien de particulier.

Toutes les personnes qui en ont mangé l'ont trouvé très bon.

Plusieurs fois j'en ai fait faire du chocolat à l'ean ou au lait, et jamais il n’a présenté de différence arec le chocolat ordinaire arrangí de la mème manière et pris comparativement.

Ce chocolat, que je pourrais appeler de santé, est convenable aux hommes, mais sur- 
tmit aux femmes qui allaitent et aux enfaus en bas âģe.

\section{Cruchude.}

Ce mets, qui ressemble beaucoup à la polenta, dont nous parlerons plus tard, en diffère dans quelques points.

Voici comme on la prépare : on fait chauffer de l'eau dans un vase dont l'ouverture soit plus large que le fond, avec une quantité de sel proportionnée au goùt de ceux qui doivent faire usage de la cruchade. Au moment où l'eau est près d'entrer en ébullition, état que l'on a soin de maintenir, l'on prend de la main gauche ce qu'elle peut contenir de farine, que l'on fait tomber dans l'eau peu à peu par un léger froissement du doight, et l'on en incorpore de cette manière une quantité suffisante pour donner quelque consistance à cette boullie, que l'on a soin de remuer constamment, depuis le moment où la première pincée de farine tombe dans l'eau jusqu'à la cuisson entière, que l'on reconnait facilement, parce qu'alors la pàte 


\section{( 274$)$}

n'adhère phus à la spatule avec laquelle on l'agite. Alors on retire cette bonillie de dessus le feu, et on la met aussitôt par portions d'une ou deux cuillerées sur des linges sur lesquels elle s'étend un peu. Pour qu'elle ne s'y attache pas, il faut employer un lingequiait servi depuis le blanchissage. Les paysans se servent ordinairement pour cet usage de la nappe dont ils ont fait usage les deux ou trois jours précédens, et dont ils ont, durant ce temps, enveloppé le pain en consommation. D'autres fois, au lieu de diviser la pâte en petites portions, ainsi qu'il vient d'être dit, ils la mettent en masse dans des paniers de paille tressée avec des écorces de ronces ou d'osiers ( qui dans le pays, environs de Bordeaux, s'appellent minets, et servent à mettre la pâte dı pain à fermenter au sortir du pétrin), garnis intéricurement d'un linge; il faut pour lors que la bouillie ait plus de consistance.

Que la cruchade soit en galette on que, laissée en grandes masses, il faille la couper par tranches pour la manger avant d'être consommée, elle est cuite sur le gril ou sur des charbons pour faire évaporer une partie de 


\section{$(2.75)$}

bean yu'elle contient. Quand elle a pris une belle couleur rousse, on peur l'aceommoder aree une sance un pen relerée.

La cruchade est un mets assez delieat lorsquïil est bien fait. J'en ai mangré somvent u jen ai fait manger à des nourrices et à de jeunes enfans qui s'en sont bien trourés.

Le defaut de certe préparation est de se conserver peu sans se courrir de moisissures, saigrir et se décomposer.

Lat preparation que font subir an maïs les Indiens de la Terre-Ferme se rapproche assez. de celle-ci.

Jean Polem nous en donne les détails dans son Histoire naturelle des Indes, fol., p. sof.

Les Indiens des iles mangeaient le maïs en grains rôtis, mais les Indiens de TerreFerme ell usent autrement. Les Indiennes, principalement, le mettent dans une pierre quelque peu creuse, "t en tiemnent une antre ronde et longue en leur main, qu'elles roulen à force de bras (comme les peintres quand ils broient leurs couleurs), et jetant de l'eau parfois, ne eessent pas de moudre. Ils font une sorte de pate de laquelle ils prenuent un 18. 
petit morceau et en font un rouleau de la grandeur de la main et gros de deux ou trois doiggts, et l'enveloppent en une feuille de la même canne de maïs ou autre graine et le cuisent; quand ils l'estiment assez cuit, ils le tirent; autrement, le font rôtir à la chaleur de la braise du feu, tellement que le rouleau de pâte s'endurcit et se fait comme pain blane, ayant la croûte dessus et la mie dedans un peu plus tendres; puis ils ôtent la feuilleen laquelle il était enveloppé pour le cuire ou rôtir, et le mangent un peu chaud, non du tout froid, car quand il refroidit il n'a pas si bonne saveur et n'est pas si aisé à mâcher, parce que plus il est froid, plus il est sec et rude.

\section{Galettes, gâteaux et pâtisseries.}

Les Indiens se nourrissent de gâteaux de maïs cuits dans des terrines; ils les mangent tout chauds avec du poivre long. En Hongrie, on en fait de petits gâteaux qu'on nomme kolatez ou koukriza mala. La pâte très fluide est mise dans un four très chaud. Leur saveur est douce et agréable. 


$$
\text { ( } 277)
$$

"Les Lispagnols, dit Parmentier, p. 253 (1), qui paraissent avoir appris des Américains cette manicre simple dapprêter le maïs, ne suivent peint partont te mème mode. Les habitans des montagnes de Saint-Andei' observent beaucoup de précautions pour cuire leurs galettes an four. Ceux de plusienrs pays de la Biscaye font antant de galettes qu'il y a de personnes dans la maison; ils se servent d'un petit gril de fer rond, sur lequel ils font prendre à chacune de leurs galettes un peu de croùte, et après cela ils les exposent devant le fen, afin de laisser achever leur cuisson. Dans plusieurs endroits des Asturies, ces galettes, très épaisses, sont abandonnées toute la journée sur des cendres à peine chaudes, recouvertes de paille et de fenilles séchées, d'où résulte nécessairement un aliment malpropre, à demi cuit, ayant un goùt de fumée détestable. "

Les gàieaux connus dans les différens pays où l'on cultive le maïs prennent des noms

(1) Ouvrage cité sur le mais. 


\section{( 278 )}

diver's suivant les pays : dans le Haut-Languedoc, on les nomme pain de millet, millusset, cussole; en Bourgogne, flamusse, miliasse.

Les Espagnols de l'Amérique du Sud font des espèces de galettes de maïs, qu'ils nomment arépas.

De Laët en indique de différentes espèces. Les excellens gâteaux que mangeaient chaque jour les seigneurs mexicains s'appelaient totanquitlaxcallitlaquelpacholi, ce qui veut dire: tortillas blanches chaudes, fabriquées dans un vaisseau approprié et couvertes d'une serviette blanche.

Ils mangeaient aussi certaines pàtisseries faites avec les sommités du maïs mêlées avec des semences de cresson et du jus de fruits écrasés.

Parmentier lit, dit-il, essayer de faire avec cette farine de la pâtisserie, mais il ne donne aucun résultat. Ceux qui lisent son ouvrage ne savent donc à quoi s'en tenir sur cet objet d'assez grande importance dans les pays où le maïs est presque le seul grain connu. 


\section{$(979)$}

Voici la manière de faire les flamusses de Bouryogne:

Prenez de la larine, que vous fates enire dans du lait et que vous pétrisser ensuite avec du beure, des aruls, de la fleur d'onanger' laisser lever pendant une demi-henre an moins dans le coin du pétrin, et metter an four sur me lenille de chou flétrie. Quelquefois on mêle un quart de farince de froment et trois quarts de farine de maïs.

Vous aure un gileau bien leve, assez legere et qui fait les délices de tous les Bonrgniguons et des Francs-Comtois.

Ayant en vue de procurer des jouissances aux riches comme des ressources aux paurres, jai voulu faire faire de la paitisserie avec cette farine, soit seule, soit mélangyede.

Je domnerai seulement ici quelques uns des résultats obtenus par M. Porcheron, rue des Saints-Pères, $\mathbf{n}^{\circ} 36$, pàtissier intelligent, qui a bien voulu se charger de ce travail.

Il a fait :

$1^{\circ}$. Des brioches aree un quart de levain et. rois quarts de farine de maïs, qui luren trources très bonnes par le pàtissier lui-mème, 


\section{( 280$)$}

et plus délicates que celles fatites avec la farine de froment.

$2^{\circ}$. Des choux aux amandes, avec trois quarts de maïs, un quart de froment, trois œufs et la valeur d'un ouf de beurre : ils étaient très bons et très légers.

$3^{\circ}$. Des petits gâteaux en pâte et confiture avec un quart fécule et trois quarts maïs, excellens.

$4^{\circ}$. Des gâteaux en triangle, en losange, inférieurs en qualité.

$5^{\circ}$. Des petits pains de Lamecy an lait, même pâte que les choux, trouvés bons.

6*. Une galette compacte, à peu près nême pàte que les brioches, très bonne.

7". D'autres galettes feuilletces, bien plus délicates.

$8^{\circ}$. Des biscuits faits à la facon ordinaire, mais avec du maïs, très légers, colorés cn jaune, excellens.

$9^{\prime \prime}$. Des madeleines, bons gâteaux.

I $^{\circ}$. Des gênoises, genre de pâtisserié préférable à celle faite avec du froment.

$11^{\circ}$. Des tartelettes à la frangipane, avec de la farine cuite, wufs et sucre, très bonnes. 


\section{$(281)$}

12". Des cornes en paite ferme : Irop firiables, sentaient la poussiere.

$15^{\circ}$. Un gàteau méme façon que les gàteaux de rì, trouvé délicieux.

14\% Un énorme gaiteau de Savoie, avec maiis, était tres jaune en dedans, conservant beancoup d'humidité, quoique très leger, et bien meilleur le lendemain, ce qui est l"inverse des, biseuits ordinaires, qui se sèchent facilement.

Les résultats, tout satisfaisans qu'ils sont pour de premiers essais faits par des ouvriers qui n’avaient jamais travaillé cette farine, enssent été, je n'en doute pas, bien meilleurs si, au lieu d'employer de la farine de gaude, javais pu me procurer à Paris de la farine de Turquieblanche et non séchée au four. Beaucoup de ces gàteaux ont eté mangés par unc nourrice et de jeunes enfans, qui ne pouvaient se rassasier; les hommes les trouvaient délicienx, et je n'aurais pu suffire à la consommation.

\section{Gaudes.}

La bouillie de maïs, que les Bourguiguons nomment gaude, a reçu en Nllematgrne le 


\section{$(282)$}

nom de sterz, en Piémont farinata; les peuples de l'Amérique la nomment sagamité. Elle leur est comnue depuis fort long-temps; car voici comme Thévenot en parle (1): "Lors de la découverte des Illinois, le 25 juin 1663 , le conseil fut suivi d'un grand festin chez le grand capitaine : il consistait en quatre mets qu'il fallait prendre avec toutes leurs façons. Le premier fut un grand plat de bois plein de sagamité, c'est à dire de cette farine de blé d'Indequ'on fait bouillir avec un peu d'eau, et qu'on assaisonne de graisse, ete."

On distingue ordinairement trois sortes de gaudes: $1^{\circ}$ gaudes simples; $2^{\circ}$ gandes à la courge; $3^{\circ}$ gaudes aux pommes de terre.

\section{Gaudes simples.}

On met de l'eau sur le feu, et quand elle est en ébullition, on y ajoute peu à peu de la farine de maïs jaune, qui aura dû être séchée au four; on remue toujours de peur que la bouillic ne s'attache an fond et ne brùle, on

(1) Recucil de Toyages. Découverles dans I'Anicrique septentrionale, p. i9, in-8. Paris, 1687. 
Y ajoute vers la fin un pen de lait, une pincér de sel et un morceau de beurre; si l'on ne se servait que de lait, ee plat serait beatucoup plus délicial.

Un mets beacoup plus simple sullit, dans certains pays, à des hommes gui fatiguent beaucoup.

"Souvent, dit Auguste Saint-Hilaire, lor'sque les muletiers brésilieus arrivent ì leur lieu de repos, et qu'ils n'ont pas la patience d'attendre que leurs haricots soient cuits, ils apaisent leur faim en mangeant un jacuba, mélange qui se fait à froid avec de l'ean, de la farine de mais et des rupaduras (espèer de sucre)."

\section{Guudes à la courge.}

Les potirons on les comryes ordinaires, cuits à l'eau ou au lait, se mèlent quelquefois aux gaudes, ils donnent ainsi un mets beancoup plus sucré et plus rafraichissant.

Gaudes it la ponune de terre.

L'andidon retiré des ponmes de terre, on 


\section{$(284)$}

leur pulpe cuite et écrasée au rouleau, se mêle, dans certains pays, aux gaudes. Cette préparation est alors bien plus nourrissante, et il en faut moins pour soutenir les forces.

Ces deux dernières préparations sont avantageuses dans les contrées où la courge et la pomme de terre entrent comme culture intermédiaire dans les rangs du maïs, parce qu'alors on a continuellement et sans rien débourser ces deux produits sous la main.

On conçoit que, si on veut pouvoir servir ces plats sur la table de gens riches, on pourra les rendre meilleurs en y ajoutant du sucre, de la crême, de la fleur d'oranger, de l'écorce de citron, de la cannelle, etc.

Quoique je ne sois pas habitué à ce genre de nourriture, pendant plus de deux mois il a fait toujours, sous une forme ou sous l'autre, la base essentielle de mon déjeûner. J'ai même fait l'expérience de me nourrir, pendant plusieurs jours consécutifs, seulement avec des gaudes cuites à l'eau, avec un petit morceau de beurre, et pour boisson de l'eau pure. Comme j’ai fait répéter cette expérience sur plusieurs nourrices et sur de très jeunes en- 


\section{$(285)$}

fans, jaurai occasion de revenio sur les résullats en parlant des effers de la nomrriture du maïs. Kalm assure que cette houillie est prépérér, clans l'Amórique septentrionale, par les Anglais et les Hollandais, aux autres graius dont ils ont d'amples provisions.

\section{Gaufres.}

Pour filhriquer leurs gaufres, les Bressans mélangent un tiers de farine de maïs avec deux tiers de blé de sarrasin; ils délaient le lout dans de l'eau avec une très petite quantité de beurre et de sel, et ils les font cuire dans les fers. Ce mets est assez insipide.

Le maïs blanc est préférable. J'ai fait faire des gaufres par le procédé ordinaire, mais avec parties égales de farines de maïs blane et de froment; elles ont toutes très bien réussi, et beaucoup de personnes qui en ont mangé les ont trouvées délicieuses.

\section{Gruale.}

Le maïs-gruau se nomme farre dans le ciderant Roussillon. 
Il vient, dit-on, à la Lotisiane un mais dit à grru ou à gruau. Son gुrain est rond, dur et luisant; il y en a de blane, de jaune, de rouge et de bleu.

Le gruau pour potage se prépare ainsi qu'il suit : on le délaie dans des véhicules nutritifs, tels que le lait, le bouillon; on le passe à travers un tamis ou un linge, et après on le fait euire.

Parmentier assure que c'est le grain le plus propre à remplir cet objet. Il ressemble au millet; il en a à pei près la couleur et le goût.

\section{Macaroni.}

M. Quentin, vermicellier, rue Montmartre, $\mathrm{n}^{0}{ }_{12}$, a fabriqué du macaroni dans lequel il a fait entrer la farine de maïs dans les proportions suivantes :

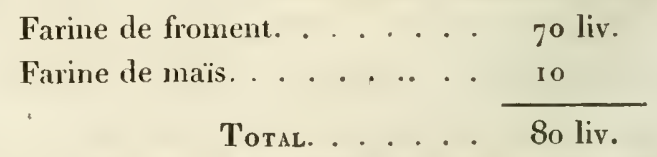

Quoique le maïs ne soit qu'en très petite 
quantite, il a commmoniqué an macamoni mus trinte jantator que fo tomps a fait dispanaitre, mais les fuyaux somt pipues de points blanes. Sa cassure est luisante. Plusieurs fois jai fait préparer avec cette pàte des plats de macaroni, et tontes les persomnes qui en mangèrent le tromirent parfait. Il était plus onctueux, bien filant et un jen plus jaune que le macaroni ordinaire. Ce mets, qui convient anx hommes, ne conviendrait que très peu aux femmes qui allaitent, surtout conme on le mange en France, c'est à dire vers la fin dı repas, lorsque l'estomac est déjà surchargé d'alimens; en toute autre circonstance, je erois qu' elles le digéreraient très bien : il faut bien se garder d'en donner anx très jemnes enfans.

Millusse-ou Milhus, voyez Cruchade.

\section{Mä̈s mondé.}

Les Américains séparent le maïs de son écorce sans le réduire en farine, soit en emplovant des moulins à pilon, qu'uu cheval fait mouvoir, soit en échaudant ee grain avec de la lessive. 
Lorsque le grain est ainsi dépouillé, on le met bonillir avec de la viande, et lorsqu'elle est cuite, la soupe est faite; elle a le goût de la soupe de pain. C'est, selon Kalm, un potage très sain et fort nourrissant.

J'avais pensé à faire monder du maïs, et même à tâcher de l'obtenir perlé, mais il n'existe pas de fabrique d'orge mondé ni perlé à Paris, toutes sont situées en Hollande; je n'ai pu vérificr les idées que je m'étais formées à ce sujet, et les usages auxquels je voulais le destiner.

Miques.

La mique se conserve plus long-temps que la cruchade, mais s'aigrit et se décompose comme elle.

Pour la préparer, on place dans un des coins du pétrin une certaine quantité de farine de maïs, au milieu de laquelle on fait un creux dans lequel on met du sel et. oụ l'on verse de l'eau bien bouillante, que l'on remue d'abord avec une spatule; après quoi, l'on manie cette pâte avec les mains, et on la durcit extrêmement, parce qu'elle a le défaut de se ramollir; 


\section{$(289)$}

On la laisse alors dans un des coins du pitrin lo lemps nécessaire pour amener l'eau dans lafuelle elle doit enire an premier degré d'ebullition, et l'on rédnit celle pitte en boules d'un diamene plus on moins grand (de 6 i 2 centimetres; en frinciral les plus petites sont celles qui cuisent le mienx et sont les plus commodes pour la consommation), en maniant la quantité nécessaire pour les former. Quelques persomuce les monlent avec de la farine de maïs; mais il en résulte (pue eette farine nowvellementincorporce, n'étant pas sullisanment imprégncie d'ean, se décompose lorsqu'on met les boules dans l'eau en ébullition, et occasione quel(quefois la décomposition entière de la pàte qu'elle envoloppe. D'autres personnes out à coté du pétrin un vase d'ean froide, dans lequel elles trempent leurs mains, et monlent ainsi leur paite, qui se conserve mieux lorspuion la met cuire.

L'eau, an moment ou l'on y met les boules, ne doit pas ètre plus échauffée qu'an premier moment d'ébullition, élat qu'on doit maintenir tout le temps de la cuisson, ee qui rend l'oprération un peu lougue. Ia formeté que 


\section{( $\left.29^{\circ}\right)$}

cette pàte aequiert en fixe le terme, et doit être la même tant à l'extérieur qu'à l'intérieur de la boule; pour s'en assurer, si on les croit suffisamment cuites, on en sort une de l'eau, on la partage en deux ou quatre quartiers, qu'on laisse refroidir, et l'on voit ainsi si elles ont acquis la même consistance dans toutes leurs parties. Quand elles ne se trouvent pas cuites intérieurement, les paysans disent qu'elles ont le fion.

Comme les couteaux ou tout autre instrument tranchant ont le défaut de se garnir de pâte, les paysans les coupent avec un brin de paille aplatie, et dont on tient entre les doigts les deux extrémités.

Il faut observer que toutes les miques soient moulées avant de les mettre dans l'eau où elles doivent cuire, parce que, sans cette précaution, les premières seraient cuites et les autres ne le seraient pas. Il faut aussi faire attention qu'elles soient couvertes par l'eau durant tout le temps de leur cuisson, qui ordinairement a lieu dans un chaudron.

Au sortir de l'eau, on les met égoutter sur' des claies couvertes de linge, et on les mange 


\section{( 291$)$}

lorsqu'elles sont froides, soit telles qu'on les a prẹparées, soit plus généralement coupées en tranches d'une plus ou moins grande ćpaisseur et rôties sur le gyril ou sur les charbons.

Suivant le gont de ceux qui doivent les consonmer, l'on ajoute à cette préparation de la farine de froment ou de seiggle, mais en fort petite quantite, Iorsque, dans le moment du pétrissage, la pàte de maïs devient maniable; on! met aussi quelques grains de fenouil, d'autres personnes n'y ajoutent rien.

\section{Pain.}

Tous les auteurs qui ont écrit sur le maïs ont été d'avis différent sur sa panification, et ils se sont divisés en deux classes : les uns prétendant que c'était plutôt un gàteau (qu'un pain, les autres soutenant au contraire quil avait tous les caractères du pain. Cette différence d'opinion d'hommes très éclairés sur un point aussi précis, dépend sans doute de la manière dont ce pain avait été préparé et aussi des ouvriers auxquels cette fabrication avait été confiée, peut-ètre 
même de l'espèce de farine employée. L'expérience seule pouvait m'éclairer dans cet état de doute : aussi, ai-je fait faire de nombreux essais, soit en employant la farine de mais seule, soit mélangée avec du froment, du seigyle, de la fécule, etc. Je crois avoir le droit de donner mon avis, puisque pendant un an environ j'ai fait fabriquer au moins 300 livres de ce pain, et que long-temps je n'en ai pas mangé d'autre.

\section{Pain sans mélange.}

Avant de parler de cette fabrication, il est utile de savoir quelle est la farine préférable.

Parmentier, qui a fait beancoup d'essais, préfère la blanche.

N'ayant pu me procurer de la farine blanche que quinze jours avant la clôture du concours, et encore a-t-il fallu la faire venir de la Bourgogne, presque tous mes essais ont été faits avec du maïs jaune, et je trouve qu'il produit un meilleur effet, et que le pain qu'il donne a beaucoup plus de goût.

M. Tessier dit, dans les Annales d'agri- 


\section{$(295)$}

culuure fronçaise, inme 11, page 332, qu'on a trouvé très peu de différence entre les pains faits de milis ì yrains jannes, blanes et rouges, séparément; leur cronte était luisante et jaune, et la mie pàteuse, compacte et plus jaune que la cronte : cette couleur avait moins d'intensité dans le pain de mais blane que dans les deux antres.

Celıi que j’ai fait labriquer l'a été par le deuxième procédé indicqué dans l'ouvrage de Parmentier, page 269.

On prend un morecan de levain réserré de la dernière fournce, on le délaie avec la moitié de la farine destinée an pétrissage et de l'eau tiède pour en former une pàte ferme qu'on placedans un lieu chaud pour qu'elle léve.

Trois heures après, on délaie ee levain avec le restant de la farine et suffisamment d'eau tiède pour en former une pàte très molle, et quand elle a été pétrie comme il convient, on la distribue dans les paniers, et ensuite on l'enfourne.

Ce pain, toujours assez pesant, était mince, aplati, et les yeux étaient fort petits; mais à force d'en laire et de changer, soit la mani- 


\section{(294)}

pulation de la pàte, soit la chaleur du four, soit aussi la quantité de levain, M. Adam, boulanger, rue du Cherche-Midi, $n^{\circ} 6$, qui a mis beaucoup de bonne volonté à ce travail extraordinaire, parvint à me faire du pain de maïs pứ, bien levé, parfaitement œilleté, dont la croûte était assez mince, enfin qui ne ressemblait en rien à ceux qu'il avait faits en premier lieu. Il était beaucoup plus savoureux et plus agréable à manger que le premier.

Une seule fois, j'ai tenté une expérience qui n'a pas réussi et qui m'a laissé les plus vifs regrets, parce qu'elle me faisait espérer d'ouvrir un nouveau genre d'industrie. Pour' faire comprendre mon. idée, je vais rappeler des faits qui s'y rattachent.

On sait que les amidonniers préparent leur amidon par la voie de la fermentation, moyen tout à fait insalubre et assez long. On sait aussi que par le lavage, on obtient de la farine de froment l'amidon qu'elle contient, et aussi le gluten qui se trouve perdu par le procédé de la fermentation.

M. le docteur Herpin avait fait mu mémoire qu'il présenta à la Société d'économie domes- 


\section{$\left(29^{5}\right)$}

tique, en 1829, pour obtenir l'amidon par le lavage et ticher d'utiliser le gluten; mais quel emploi lui donner? Je m'imaginai de l'extraire d'une livre de froment et de le faire incorporer dans une livre de farrine de maïs pour en faire du pain; car ce qui empêche cette farine de lever, e'est l'absence nulle ou presque nulle de cette substance animale dans ce grain. Malheureusement le pain qui en résulta etait lourd, humide, gras, très conpacte et peu mangeable. Si l'époque du concours ne füt pas arrivée, j’aurais réitéré cet essai, mais en le variant, c'est à dire en faisant dessécher mon gluten et en le réduisant ensuite en poudre; cette préparation nouvelle, si elle réussissait, occuperait sans doute quelques bras sans ouvrage ( 1 ).

En Italie, on fait de petits pains ronds de farine de maïs pur que l'on vend daus les villes; ils servent aux ouvriers qui les mangent le matin en buvant l'eau de vie, ou avec

(1) De nombreuses occupations miont empeché de suivrece premier essat; mais jespèce pouvoir contumer mes recherches. 


$$
\text { ( } \left.29^{6}\right)
$$

des tranches de lard frit qui se vendent sur lesplaces. Ce pain est de mauvais goût pour un Francais, mais le peuple italien le trouve bon.

Le maïs est en usage parmi les habitans de l'Orénoque; ils en forment de petits pains lonģs, mais ce n'est qu'une pâte entourée de feuilles, qu'on fait bouillir.

Les Tamanaques donnent à ce pain le nom de camaitcà. Chaud, il n'est pas mauvais, et beaucoup d'Espagnols en font usage.

\section{Pain mélangé.}

On peut mélanger le pain de maïs avec presque tous les grains ou tubercules : on l'a fait pour le froment, le seiggle, la pomme de terre, l'orge, le sarrasin, l'avoine, la vesce, les haricots. Je n'ai fait des essais qu'avec le froment, le seigyle et la pomme de terre.

Les habitans des campagnes de la Haute et Basse- Égypte trouvent dans le maïs leur principale nourriture, et ils en font du pain mélangé avee la farine de froment et de fenugrrec.

Les Nègres de la còte d'Or font du pain de maïs pur, ou en le mélang̣eant avec du millet. 


\section{$(297)$}

P'ain mélangé avec du froment.

Quand on le peut, c'est sous forme de levain que le froment doit ètre incorporé arec la farne de mails.

Les premiers pains qui me furent fits avec ces deux firnes, à parties égales, étaient lourds, compactes, détestables enlin, et cela parce gue le boulanger, voyant qu ils nelevaient pas, y ajoutait toujours du levain nouveau; il remaredat ensuite qu'il fallait agir en sens inverse, et il obtint du pain bien meillens. Depuis, le froment est entré dans toutes proportions possibles daus ce pain, et les résultats ont toujours été siltisfaisans. Cependant j'ai cru que le milange par parties égales citait celui qui donnerait le pain le plus avantageux et le plus beau.

Prriu mélungé avec moitié de seigle.

Ce pain est lourd, compacte, el garde beaucoup d'humidite.

Dans le nord de l'Amérique, le prin de 


\section{$\left(29^{8}\right)$}

ménage est ordinairement mi-parti de farine de maïs et de farine de seigle. M. de Rumford doute qu'il soit possible de trouver uu pain plus salubre et plus nourrissant.

Si on ne mélange le seigyle que dans la proportion d'un quart, ee pain devient très bon et très nourrissant; il est d'un usage ordinaire en Italie. Les belles paysannes du Verceillais, qui en mangent tout l'hiver, sont bien portantes et d'une haute taille.

Pain mélangé avec du froment et du seigle.

Ce pain, lorsque les proportions en sont gardées par tiers, est assez beau; sa couleur est gris-jaunâtre. Le goout de seigle domine beaucoup sur celui de maïs; il se conserve frais très long-temps.

Pain mélangé avec de la fécule de pomme de terre.

Par partie égale, ce pain, fait avec de la farine de maïs blanc, était d'un blane légèrement grrisàtre; il était très bien levé, léger, 


\section{( 299 )}

mais son senl défant étaic de sentir trop lo grocit de pomme de terre. Avec ce pain surtout, on pouvait trés bien tremper la soupe.

Le mais mélangé, par parties égales, avec du froment el de la pomme de lerre, donne. un pain hien levé, plus hlane et plus savourelix.

Le pain composé de maïs une partie, fronent une partic et une demi-partic de fécule, pain que je n'ai fait faire qu'une seule fois, n’a pas hien réussi : il était lourd et très sec.

Enfun jai roulu voir si, en faisant cuire préalablement la farine de mais, le résulta1 serait satisfaisant.

Une live de mais fut réduite en boullie, et dans cet état, lorsqu'elle fut refroidic, on la mélangea avec partic égale de froment; mais le pain en était gras, très humide et mauvais, en le comparant avec un autre pain fait avec le mème mélange par un autre procéclé.

Le programme publié par la Socicté d'horticulture, en 1850 , dit, page 4: Le pain qu'on en fait est excellent, mèlé avec mu tiers de farine de froment. Moitie maïs, moitie orgy' at un cincuieme de farine de froment don- 
nent un pain aussi beau que le meilleur pain de seiggle mélangé avec le froment, et si l'on veut mêler à parties égales le froment avec le maïs, on obtiendra le pain le plus agréable et le plus succulent qu'on puisse désirer.

Parmi les mélanges qui ont paru réussir le mieux ou offrir des vues d'économie, dit Tessier, on voit le suivant: froment, maïs, orge et vesee, de chaque un quart, la vesce privée de son amertume, en la laissant dans l'eau quelque temps; on peut remplacer la vesce par la lentille, la gesse, les pois ou les féveroles.

Lorsqu'on se propose de faire du pain de différens grains, il faut plutòt faire le mélange des grains que celui des farines : l'union est plus intime et plus complète par l'action de la mouture.

Les pains dans lesquels entrent le seigle, l'avoine, le maïs, le sarrasin et le haricot ont besoin d'être au four plus long-temps que les autres; le 'temps doit être proportionné à la dose de ces grains. Une cuisson lente leur convenant mieux, il est nécessaire que le four ne soit pas trop chaud. 


\section{(301)}

Si l'on voulait rendre le pain de mais plus nourrissant, on pournait y faire entrer la grelatine en proportions diverses.

11. Limer, boulanger, rue de Richelieu, fabrique, depuis janvier 1850 , des petits pains de maïs tris hien faits et tris hons, dont il at un grand dibit. Les mus sont simples, c'est ì dire saus mélange, les autres au beurre. Quelques uns sont mélangés d’anis ćtoilé, rédnit en poudre fine; enfiu d'autres contiennent de l'huile essentielle de citron el dui snere, on des raisins sees.

De tour ceci, je ne puis que eonchure que, par un travail sontenu, on parviendra a faire d'assez bon pain avec le mais pur, et d'exeellent pain avec le maïs mélingé en différentes proportions avee les autres farines.

Lorsqu'on l'anra ohtenu bien levé et léger, il remplacera le pain de froment daus les pays où ce grain est rare, et où il faut le faire venir ì grands frais.

En tous eas, comme je l'ai obtenu, et presque sous tontes les formes, sauf les exceptions que jai indiquies, il convient mieux que le pain de froment aux hommes qui se livrent à 
de durs travaux, aux femmes qui doivent fournir un lait très abondant à leurs nourrissons, et il plaît beaucoup aux jeunes enfans qui le préfèrent au pain blanc de froment qui est toujours fort insipide, parce que, dans le pain ordinaire, l'on a voulu obtenir sa blancheur aux dépens de sa bonté.

\section{Pain d'épice.}

M. Cotty, fabricant de pain d'épice, rue du Four-Saint-Germain, $\mathrm{n}^{\circ}$ 63, a bien voulu se charger de faire quelques essais avec la farine de maïs.

Le 26 novembre 1829 , il fit du pain d'èpice avec la farine de maïs pur. Ce pain d'épice a mal réussi ; il est beaucoup trop sec, et n'est cependant pas désagréable à manger.

Le is décembre 1829 , il en fit d'autre avec moitié farine jaune de maïs et moitié seigle; il n'a pas réussi davantage, quoique le produit obtenu fùt plus levé et moins crevassé.

Le 14 janvier 1830 , il fit deux noureaux essais. 


\section{$(505)$}

Dans le premier, il mit moirie mais jame "1 moitié seigrle, mais moins de levire. Il laissa moins de temps ì lever, et il obtint des résultats beaucoup plus satisfitisans.

Dans le denxieme essai, il employa moitic maïs blanc et moitié froment. Le produit est plus heau que le précédent. J'ai possídé des échantillons de ces derniers essais pendant m an; ils se: sont hien conservés et ils étaient aussi hons que le premier jour.

En modiliant ce travail, peut-être pourraiton espérer plus de succés.

\section{Pets de nonnes.}

Pour les préparer, prenez une petite mesure de lait, 4 onces de suere, une petite quantiti de fleur d'oranger, trois cuillerées de farine de mais; batiez bien le tout en ajoutant la farine peu à peu. Mettez les jaunes de deux ou trois oufs, battez les blanes à part, et quand ils seront bien levis, ajoutez-les par portions dans votre mélange, en évitant de mettre la partic liquide qui se trouve au fond du plat; agite\% bien le tout, et jetez par cuillerées dans de la 


\section{(304)}

friture très chande et fraiche; ce mets est très bon.

$\mathrm{Si}$, arant d'ajouter vos œufs, vous placez votre pàte sur le feu et la faites cuire pendant vingt minutes, que vous laissiez refroidir un peu avant d'ajouter vos œufs, et que vous battiez hien, rous aurez, en contimuant comme ci-dessus, un plat beaucoup plus léger et plus délicat que le précédent.

Cette préparation, ainsi faite avec la farine de maïs, l'emporte beaucoup sur celle que l'on fait de la même manière avec la farine de froment. Cette nourriture plaît beaucoup aux hommes, aux femmes qui allaitent et surtout aux jeunes enfans, car c'est une véritable friandise.

\section{Polenta.}

Si l'on r'emonte à l'origine de cette préparation, on voit qu'elle est fort ancienne. Lorsque les Indiens voulaient se servir du maïs, qu'ils cultivaient avant l'arrivée des Européens en Amérique, ils brovaient le grain avec des pierres on des pilons en bois, et en tiraient une farine assez grossière. Ils la faisaient ensuite 
bouillir a l'aide de pierres rouggies an fen qu'ils jetaient dans de l'eau jusqu' ì ce que l'ébullition eùt lieu (1).

En Italie, en Espagne et dins les départemens méridionaux de la France, les paysans composent, avec la farine de maïs, un mets (quils appellent polcnta. Cette nourriture est très économique, sa préparation est très prompte, elle se diggere facilement, elle est préférable à la plupart des fícules. En Italie, presque tous les paysans n'ont d'autre pain que cette espèce de bouillie épaisse. Ils ne font pas un repas sans polenta; c'est leur pain, ils n'en connaissent pas d'autre.

Il n'est aucune famille qui n'ait une espèce de vénération pour le chaudron et le bàton héréditaires. Je vais donner la manière de se servir de ces meubles précieux, car il en est qui comptent plusieurs générations, c'est le bàton patriarcal, et heureuse la famille qui peut montrer comme titre de noblesse ces deux objets chéris, provenant en ligne directe du règne de tel ou tel prince.

(1) Warden, ouvrage cilé, 1. 102. 


\section{$(300)$}

Ce fait parait étonnant, vu l'usage journalier d'objets fragiles, mais en roici l'explication. Le bâton patriarcal est une espèce de crosse, longue de trois pieds, ayant un pouce et demi de diamètre. Pour s'en servir, on le tient de la main gauche renversée et peu serrée, de manière qu'il puisse tourner facilement; la main droite, opposée à la gauche, le tient plus bas, hors du chaudron : c'est de cette main qu'on appuie fortement dessus pour le faire tourner, mouvement que lui fait prendre aisément son gros bout, qui, appuyédans le mélange, forme une courbe; on tourne toujours de l'extérieur à l'intérieur. Quoique ce bâton serve au moins quatre fois par jour, il se conserve, parce qu'il n'est jamais gyratté, lavé ni essuyé d'aucune manière. La pâte qui l'entoure n'étant jamais sèche, par son usage continuel, y reste adaptée et formc une croutte qui le conserve; seulement, au bout de longues années, il raccourcit par suite du frottement au fond du chaudron. Il a sa place fixe dans la maison, et ce serait $u n$ crime de le déplacer. Le chandron est de cuivre de la plus forte épaisseur ; il n'esı jamais nettoyé avee des matières minérales; 


\section{$(307)$}

lorsque la polenta est faite, on y met de l'ean pour détremper la pâte qui adhère, et on l'écure avec des herbes. II est mis en place commu: le bàton, et n'en sort que pour' son office. Les femmes sont toujours chargées du soin de faire la polenta; il faut quiun homme soit seul habitant dela ma ison pour qu’il prenne cette peine.

Pour faice la polenta, on met le chaudron sur le feu, contenant une quantité d'eau toujours égale. Lorsqu'elle est bouillante, on verse la farine et on remue avec le baton jusqu ì ce qu’elle soit bien cuite : elle a alors pris l'épaisseur voulue; elle est très ferme. On la renverse sur un linge très blanc, et les tranches se conpent arec un brin de fil attaché à deux petits bâtons; on la mange toujours chaude; il n'y entre absolument que du sel qu'on a mis dans l'eau an moment de l'élutlition.

J'ai plusieurs fois mangé de la polenta faite ainsi, el quoiqu'elle paraisse lourde, elle se digrère facilement et nourrit beaucoup. Ce que nous venons de dire prouve assez que les femmes cui allaitent et les jeunes enfans n'ont pas une nourriture ì part. Hommes, fenmes, enfans, tout le monde s'en trouve bien. 


\section{( 308 )}

J'ai dit que le maïs nourrissait beaucoup; l'opinion de M. Grégory (I) me serait utile, s'il ne m'était facile d'en donner des preuves nombrenses.

Les charpentiers, scieurs de bois et tous ceux qui font les plus pénibles travaux dans la journée, nous ont souvent dit qu'avec 3 livres de farine de maïs réduite en polenta, qu'ils mangent ordinairement avec du fromage, du moût de raisin cuit ou du miel, ils pouvaient résister au travail, tandis qu'arec du pain blanc du même poids, ils ne pouvaient supporter le travail sans avoir un potage et du vin.

On fail aussi de la polenta dans les villes d'Italie, mais celle-là est un plat de luxe qui revient assez cher.

Lorsque la bouillie est faite, dit Parmentier, on la coupe par tranches minces, de l'épaisseur de 2 lignes; on les étend dans une casserole, en mettant du beurre et du fromage de Parmesan à chaque couche, et par dessus le

(I) Annales de l'agriculture française, IIIe série, III' vol., p. $9^{3}$. 


\section{$(5 \circ 9)$}

tout une seule couche de poivre, de cannelle et de girofle en poudre.

Les Milanais, qui sont très friands de ce mets, y ajoutent, suivant les circonstances, des foies gुras, des crètes de coq, des truffes noires, des jus de viandes : tout ce qu’ils imaginent, en un mot, d'agréable et de recherché.

Plusieurs fois, et toujours en variant l'assaisonnement, j'ai fait faire de ce plat; il faut en avoir mangé pour savoir quel goùt il peutavoir, il est vraiment difficile d'exprimer quelle en est la bonté. Ce mets, trop excitant, ne conviendrait pas aux nourrices et aux jeunes enfans.

Dans les hautes vallées et les montagnes de l'arrondissement de Port-Maurice ( 1 ), les hitbitans ne récoltent le blé que pour le vendre; la nourriture se compose de chàtaignes séchées à la fumée, de légumes et de polenta. On mange de la viande les jours de fète seulement. Le pain se fait d'un mélange de seigle et de froment; on en consomme très peu.

Et plus loin, page 33r, il est dit : Dans tout

(1) Statistique due departement de Montenolle, L. I. p. 33o. 
le reste du département, ce sont les chàtaignes et le maïs qui nourrissent les trois quarts de la population.

Dans la polenta qui se fait à la manière ordinaire, on ajoute quelquefois un peu d'huile et des figures sèches.

\section{Potages économiques.}

C'est Chomel qui, le premier, a décrit ces potages économiques; il en parle dans son dictionnaire, et indique les services qu'on en tira après les disettes de 1693 et 1709 . M. de Rumford fournit la recette suivante pour la soupe la moins coîteuse que l'on pût préparer en Angleterre :

Prenez 8 gallons d'eau, mêlez-y 5 livres de farine d'orge, et faites réduire le tout par la cuisson en une épaisse gelée; assaisonnez-le avec du sel, du poivre, du vinaigre, des herbes et quatre harengs saurs pilés au mortier; ajoutez-y, au lieu de pain, 5 livres de maïs, sous la forme de samp; remuez ce mélange avec une cuiller, el servez-le par portions d'unc livre et demie. 


\section{( III)}

Le samp, dont limmlond recommande l'usalge, est 111 mets de l'invention des samvayges d'Amérique, qui sont privés de moulins. Lat maniere de le préparer consiste à deponiller le mais de son enveloppe exterieme en le faisam ramollir pendiunt dix ou doure hemes daus une lrssive d'ean el de cendres de bris; par ex moyen, l'enveloppe se sépare du grain el nayre. sur l'au, landis que le grain demeure an fond dn vase. On fiar enire lentement ce gra dépouille, rn le laissint quelques jours do suite dans une chambiere avee de l'ean, i quelque distance du fen. Lat cuisson acherée, les grains s'enflent extritordinairement ot erè vent; ils ont. alor's une saveur trés donce, sont très nourrissans et se mangent de plusienrs manieres : la meilleure est de les mèler avee du lait, des soupes ou du bouillon, au lieu de pain. Le samp est mème préférable au pain sous ce rapport, car il flatteautant le gout que le meilleur pain, et comme, sans être d'une dureté désagrrable, il ne s'amollit pas avee antant de promptitude dans les liquides, il augmente et prolonge le plaisir que l'on trourc à manger, en ce quil exige une plus longue mastication 


\section{( 312$)$}

Le potage, composé de la quantité d'ingrédiens mentionnés dans la recette précédente, suffira pour soixante-quatre personnes et coûtera; savoir :

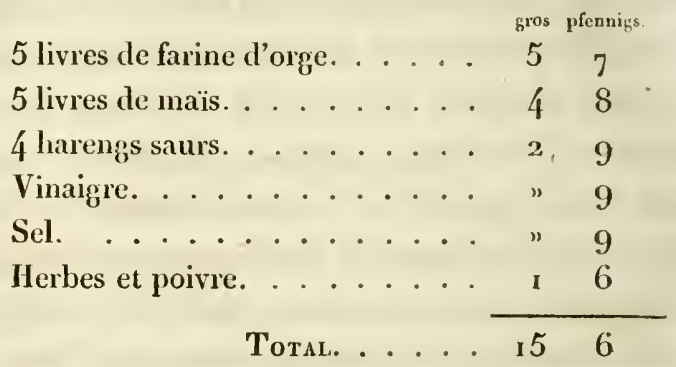

Ces 15 gros 6 pfenniggs, divisés par 64, donnent pour chaque portion un peu moins de 3 pfennigs.... Mais dans le temps où l'orge et le maïs coûteront quelque chose de moins, chaque portion d'une livre et demie ne reviendrait qu'à 2 pfenniggs. Il y a des pays où des soupes assaisonnées de vinaigre, de sel et de harengs pilés ne feraient pas fortune.

A Turin, par exemple, lorsqu'on a voulu introduire ces potages économiques, il a fallu changer leur composition, substituer le riz it l'orge, doubler la dose du maïs, et, au lieu de harengs, de sel, de vinaigre, employer 


\section{(313)}

des sulbances grasses el onctucuses, comme l'huile, le lard, avee des stimulans, tels que l'ail, la civette, les fruits du piment ammel et du poivre-corail (I).

Quant aux potages ordinaires faits avec du maïs, un peu de farine de gande, mise daus la soupe aux herbes, remplace avantagreusement et beancoup plus promptement la purće de pois et de lentilles. Elle donne à cette soupe plus de consistance, et la rend bien plus savoureuse.

Si l'on veut des potages plus recherchés, je dirai que, plusieurs fois, jai fait faire, avec cette farine et par les procédés ordinaires, une purée aux croùtons qui s'est toujours trouvée très bonne.

\section{Pouding.}

Burger (2) nous indique la préparation du pouding suivant la recette de Rumford.

(1) Franç. de Neufchiteau, ouvrage cité, p. 238 et 320 .

(2) Ouvage cité (en allemand), P. 353 et suiv. 


\section{$(314)$}

On mange ce mets avec beaucoup de plaisir dans toute l'Amérique; on lui donne le nom ,de hasty-puding (gàteau fait à la hàte, tôtfait). On met sur le feu, dans un pot ou unc chaudière de fer découverte, la quantité d'eau nécessairepour un pouding; on y fait dissoudre ce qu'il faut de sel, et l'on y mêle peu à peu la farine de maïs avec une cuiller de bois, dès que l'cau est chaude et commence à bouillir. On ne fait couler successivement dans l'eau qu'une petite quantité de farine, à travers les doigts de la main gauche, tandis que de la main droite on imprime à l'eau un mouvement précipité pour que la farine s'y mêle complétement et pour l'empêcher de se former en paquets. Au commencement de la cuisson, il faut introduire la farine avec beaucoup de lenteur, afin que la masse ne soit pas plus épaisse que de la soupe de gruau d'avoine. L'addition du surplus de farine nécessairc pour donner l'épaisseur convenable au pouding demande à être reculée au moins d'une demi-heure, durant laquelle il faut tenir sans cesse la masse en mouvenent et en ćbullition. On s'assure si le pouding a l'épaisseur conve- 


\section{$(315)$}

nable "u cufoncant la cuiller de bois an milien de la masse. Si clle n'y demenre pas debout, il faut rneore ajouter de la farine; mais, dans le eas contraire, le pouding est ee qu'il doit ètre et il ne fiut plus de farine. II n'en vaudra que mieux si on le laisse cuire trois quarts d'heme ou une heure entière an lien d'une demi-heure.

Ce pouding se mange de plusieurs manières. Pendant qu'il est encore chaud, on en met des cuillerées dans du lait et on les mange arec la cuiller, au lieu de pain; il est très agréable mangé de cette manière.

On le mange aussi tout chaud avec une sance de beurre et de sucre brun ou mélasse, en y joignant, si l'on veut, quelques gouttes de vinaigre.

Ce mets, que j'ai fait préparer comme il est indiqué et que j’ai mangé de toutes les manières, ne m'a paru agréable qu’au lait.

An beurre et au sucre, som aspect n'a rieu d'appétissínt, et il mait semblé trop doux. Apress y atroir ajouté quelques gouttes de vinaigre, je l'ai trouve meilleur; mais cependant il etait pen du pont des personnes qui en 


\section{$(3,6)$}

ont mangé avec moi. Il faut, je crois, être habitué à cette nourriture.

J'ai d'ailleurs suivi, pour le manger, toutes les façons que nous indiquent les Américains. Ils le mettent tout chaud sur un plat, en observant qu'il soit égal partout; ils font un trou au milieu avec la cuiller, y mettent un morceau de beurre de la grosseur d'une noix muscade, et secouent par dessus une cuiller remplie de sucre brun ou mélasse. Le beurre, en se fondant à la chaleur du pouding, se mêle avec le sucre et forme une sauce, qui demeure dans le trou formé au milieu du plat. On mange alors le pouding à la cuiller, et l'on trempe chaque morceau dans la sauce. Pour ne pas détruire trop tôt le trou qui la contient, on prend toujours les morceaux vers le bord du plat, en avançant peu à peu vers le centre.

\section{Poudres alimentaires.}

Les habitans de l'Amérique septentrionale, 'qui sont obligés d'entreprendre tous les ans de très lonģs voyagges, ont imaginé des poudres et des pâtes nutritives qui, ćtant condensées 


$$
(317)
$$

et réduites en un petil volume, peurent aisiment se transporter pour sustenter les chassems quand ils somt malhememx ou séparés de tonte hahitation par des distances immenses. Les sauvages de Susquehanmah, au delà de Philadelphie, ont une poudre mutritive, qu'on nomme poudre verte : alle est composée de blé d'Inde torrélié, de racine d'angélique ct. d'une certaine quantité de sel commun; une cuillerée suflit ì une persomne pour sa nourriture d'un jour.

Les Lapons, les 'Tartares, les Maures et plusieurs nations errantes ont aussi leurs pàtes alimentaires. Le kacha des Tartares est, en ce genre, la meilleure composition que l'on comnaisse.

Nos anciens sauvages d'Europe connaissaieut aussi l'art de préparer ces poudres, ainsi qu'on le voit par un passage de l'Abréviateur de Dion Cassius, lorsqu'il parle des bretons.

Ils préparent, dit-il, une certaine nourriurre si propre à soutenir les forces, qu'après en avoir pris une quantité égale à celle d'une five, ils ne sentent plus de faim ni de soif. 


\section{( 318$)$}

La poudre nutritive inventée prétenduement, en г755, par Bonëbe, ehirurgien du régiment de Salis-Grison, n'était aussi que du blé d'Inde, broyé, grillé, mêlé de sel et d'une graine carminative qu'on croit être le cumin. Il est clair que cette recette a été copiée sur le procédé des sauvages de l'Amérique septentrionale. Il nourrit un adulte et le mit en itat de soutenir des travaux pénibles, à la dose de 6 onces par jour, dans un demi-setier d'eau, à 5 onces par repas, selon les expériences authentiques qui en ont été faites à l'hôtel royal des Invalides, au mois d'octolsre 1754 .

On en a aussi fait l'expérience sur plusieurs soldats, la plupart jeunes et vigoureux et de bon appétit, qu'on a nourris pendant quinze jours de cette poudre alimentairee. Ces soldats ont fait pendant ce régime plusienrs exercices, ne se sont nullement sentis d'aucune incommodité d'un aliment si nouveau, ne désiraient point autre chose et quelquefois ne prenaient point leur portion entière. 


\section{$(519)$ \\ Pures.}

Les farineux, comme pois, lentilles, lat ricots, etr., cuits et ridnits e'n purée, forment IIII mets asse\% agréablile el dont on use asse\% somvent. Quelyuefois ces grains sont vendus róduits en farine, et par conséquent lem préparation est plus facile. J'ai pensé que la farine de maïs, étant très savoureuse, aromatlique et ayant assez de eonsistance, pourrail servir aux mêmes usaģes. J'ai done fait préparer plusieurs fois de la purée de maïs par les procédés ordinaires, soit avee du beurre, soit avee de la graisse de volaille, quelquefois mène en y ajontant des fines herbes. Ce mets a itri trouvi trés bon; les enfans surtout l'aiment beaucoup.

Madame Chauveau de la Miltiere combinait les substances de la ponme de terre, du maïs, de la lentille et des autres légumes, de manière à en composer des farines qui faisaient une purée excellente, et cuite an hout d'un ypart d'heure. 


\section{$(520)$}

\section{Samp.}

Voyez potages économiques, p. 3 го.

\section{Salces.}

Dans toutes les cuisines, pour préparer les sauces blanches avec lesquelles on mange ordinairement les asperges, artichauts, chouxfleurs, etc., on se sert de farine de froment; mais cette sauce tourne très souvent assez promptement. J'ai fait faire nombre de fois les sauces blanches avec de la farine de maïs : cet inconvénient s'est moins reproduit, et la sauce ayant plus de corps et de couleur, a été unanimement préférée à celle faite avec dı froment.

\section{Semoules.}

Parmentier dit fort peu de choses des semoules de maïs, quoiqu'il recommande vivement leur emploi chez les individus de constitution faible, etc.

J'ai fait faire différentes sortes de semoule de maïs par le seul fabricant qui se trouve à 
Paris, M. Bezardin, rue Bourhon-Villenewre, $10^{\circ} .58$.

Les premier's essais présentèrent beaucoup de dillicultés ef des risultats peu avantageux; mais sur mes instances, il voulut bien les recommencer, et quoiqu’il n'eùt pas le grain (füil lui fallait el préparé comme il est nécessaire pour ce travail, il a obtenu quatre espèces de semoule qui sont rangées par grosseur.

J'en ai employé en potages, et toutes les persomnes qui en ont mangé les ont trouvés Irès bons, mais ont pensé, comme moi, que la semoule faite avec dı grain de l'annce eût donné un goût bien plus savourenx que celle qui faisait le sujet de notre essai et đui avait été obtenue avec du grain de 1829 , e'est à dire de deux ans.

On en Irouve toujours chez M. Porcheron, marchand de pâtes, passage Choiseul, qui en rend beaucoup.

\section{Tomton.}

Les eréoles de Surinam préparent, avec le maïs et de la viande salée, une espéce de bou- 
din qu'ils trouvent excellent; ils le nomment tonton.

\section{Tôt-fait.}

Dans les Cévennes, dit Bosc, on donne le nom de milhas ou millias à une autre préparation de farine de maïs qui ne diffère pas en principe de ce que l'on appelle tôt-fait dans une partie de la France; mais là, c'est un mets de luxe et de réggal.

Pour faire cette espèce de milhas, on remplit de lait un plat creux ou une to urtière; on jette dans ce lait trois, quatre, cinq, six jaunes d'œufs, selon la grandeur du plat; on y met du sel, du sucre et de la fleur d'oranger, dans la même proportion, quelquefois des feuilles de laurier, de la muscade, du zeste de citron; et puis on bat le tout avec une cuiller, en y introduisant petit à petit autant de poignées de farine de maïs qu'il y a d'œufs. Le blanc des oufs a été vivement battu à part, et on ue le mêle à la masse totale que lorsqu'on est prêt à mettre le tout sous le four de campagne. Le plat est ensuite mis sur un pe- 


\section{$(323)$}

tit feu et recouvert d'un four de campagne très garni de charbons allumés.

Le blanc des aufs se gonfle, la farine cuit et se colore it la surface, et au bout d'un quart d'henre on pent servir.

Ce mets est trés bon sans doute; mais son défaut, c'est que la farine n'est pas assey cuite: j'ai done imaginé de faire cuire la farine pendant une demi-heure, avant de l'employer, et ensuite de la laisser un peu refroidir et de continuer la préparation comme il est indiqué plus haut. Autant lé premier plat est lourd, si je puis m'exprimer ainsi, autant l'autre est léger et délicat : c'est surtout préparé de cette manière, que des nourrices et de très jeunes enfans en ont mangé avec plaisir. C'est un vrai plat de luxe, qu'on pent servir sur les meilleures tables.

\section{Maïs-vermicelle.}

Parmentier dit, page 258, "quit a suivi, chez un de nos vermicelliers les plus intelligrens, toutes les opérations de son art, en variant la température de l'ean pour pétrir, depuis l'état naturel jusqu'au degré bouillant, 


\section{( 324$)$}

sans pouvoir donner à la pâte cette continuité tenace et glutineuse, dont elle a besoin pour s'allonger sans se rompre, et se prêter aux différentes formes que prend la portion de farine de froment appelée gruau, et qui s'obtient au moyen de petites meules et par une mouture ronde.

"Il s'est déterminé ensuite à mêler de la farine de maïs avec partie égale de gruau de froment, et quoique ceux-ci eussent perdu par ce mélange un peu de leur corps ordinaire, il n'en est pas moins résulté un vermicelle excellent, savoureux, de couleur jaunâtre, qui cuit très bien dans le bouillon ainsi que dans le lait, sans avoir cependant la viscosité du froment, ce qui est peut-être un avantage, parce qu'il arrive souvent que les médecins proscrivent la bouillie de ce dernier grain uniquement à cause de cette viscosité, indigeste dans beaucoup de circonstances et surtout dans les maladies d'épuisement. "

Jc m'étais adressé, pour répéter ces expériences, à M. Quentin, vermicellier, rue Montmartre, $n^{\circ} 12$; mais il m'a affirmé qu'il ne pouvait pas faire d'essai à moins de 75 li- 
vres de pàte: la dépense excessive que cette. épreuve m'eùt coùté m’a fait abandonner ce projet. A cette occasion, je ne puis que renouveler les regrets que le ghuten tiré du froment n'ait pas réussi dans la. fabrication du pain. (Toyez cet article.)

Note communiquée par un de mes confrères et ami, M. le docteur \вец Hibon, de l'Ile-Bourbon, sur la culture et l'emploi du maïs dans cette ille.

Avant la plantation dn mais, on fait remuer la terre par les noirs avec la gratte, instrument assez semblable à notre houe, mais qui porte un manche beancoup phus long.

Le mais se plante en dicembre : il serait trop tard en janvier. Pour cela, on fait des trous à la distance de 2 pieds; on met quatre à cinc graius dans chaque : il sort de terre au bout de quatre ou six jours. Lorsqu'il a atteint 6 à 8 pouces de hauteur, on le dégarnit, c'est à dire quon ne laisse que 5 pieds par chaque touffe; il faut douze à quinze jours pour qu'il arrive à cette grandeur. 


\section{$(326)$}

On débarrasse le maïs des manvaises herhes en faisant gratter la terre, et on le garnit de terre par le bas. Dans les bonnes terres, il va jusqu'à 8 pieds de hauteur; près du rivage de la mer, du côté de Saint-Leu, il est beaucoup plus petit.

En juin, les épis sont bons à ètre cueillis, et on reconnait l'époque de la récolte lorsque le pied de la plante est desséchée et a une couleur jaune-paille ( $\mathrm{I}$ ).

On casse les épis pour les séparer des tiges, et on les met dans des sacs qui sont entassés dans des magasins pour attendre le moment de la pilaison.

J'oubliais de dire qu'on plante dans l'intervalle des rangées de citrouilles, de melons, etc., et deux espèces de pois (haricots) appelés pois du Cap et pois de Bembelock : ils sont très bons à manger en vert, et très propres, étant sees, à la nourriture des animaux ;

(1) Ceux qui voudront des détails plus étendus sur la culture du mais à l'sile Bourbon pourront consulter le mémoire de M. Villèle inséré dans les Annales de l'agriculure francaise, t. XIV, p. 170. Paris, an XI. 


\section{$(527)$}

ils ont encore l'inestimable avantage de bien couvrir lat tere, de la préserver du soleil, d'y entretenir de la fratichenr et de beanconpl'améliorer'.

Pour le piler, atin de débucher les grains des épis, on se sert d'un mortier de bois de la capacite de 5 a 4 boisseaux; on le nomme pilon, et l'instrument qui sert pour piler s'ap- pelle calou: il est longde / pieds, el plus gros a ses extrémités; souvent une senle piece de bois présente quinze à vingt de ces pilons. Le' service en est fail par des noirs placés des deux còtés de la prièce, et vis à vis l'mu de l'antre, de telle manière que deux noirs pilent dans le même trou; ils chantent tous alor's une espece de refrain dont ils suivent la cadence en travaillant : cadence tellement disposée, que les calous d'un còté sont élevés, tandis que les autres frappent les épis de maïs, et vice versä.

Lor'sque les grains sont séparés des cotons (c'est ainsi qu'on appelle le réceptacle auquel sont attachés les grains), on les met dans des saes de 100 livres, et ils sont ainsi propres à ètre livrés all commerce. 


\section{$(328)$}

\section{Eimploi.}

Lorsque le mais est formé, mais qu'il n'est pas parvenu à toute sa maturité, on mange les épis grillés. On reconnaît qu'il peut être bon à cet usage lorsque la barbe est flétrie; il est nommé alors maïs grillé, mä̈s vert. Avant cette époque, on dit que le maïs est en lait, parce que les grains sont tendres, et qu'en les écrasant il en sort un liquide d'apparence laiteuse.

Le maïs sert de nourriture première, surtout pour les noirs.

Pour l'employer, on le monde entre deux meàles placées l'une au dessus de l'autre. Ces meules sont très pesantes et formées d'un roc très dur; elles ont 18 pouces de diamètre sur 4 d'épaisseur. La meule inférieure est scellée d'une manière fixe : all centre de ce disque, est placé un pivot de fer carré, dont l'extrémité est cylindrique, et surpasse le niveau de la meule d'environ 2 ponces. La meule supérieure est percée d'un trou central de 3 à 4 pouces de diamètre, par lequel on introduit le maïs. 


\section{$(529)$}

Le dessus de cente menle est garmi d'une lane de fer assez forte, qui traverse l'endroit où elle est percée, et dépasse assez le trou de la menle des deux còtrs, pour venir s'ineruster dans la roche, et y ètre lixée solidement avec du plomb. Cette lame de fer n'a pas plus d'un pouce de largeur, de sorte qu'elle laisse de chaque còté la place nécessaire pour faire passer les grains de mais; elle est percée au milieu d'un trou suflisant pour recevoir le pivot de la meule de dessons. Les deux meules doivent se toucher légèrement, et celle supérieure tourner sur l'autre, an moyen d'un manche long de 8 à r o pouces, placé près de la circonférence de la menle supérieure; par le moyen des rondelles que l'on place sur le pivot fixé dans le disque inférieur, on donne le degyré d'élévation néeessaire à la meule. Une poignée est en dessus : une senle main suffit pour ce travail, et pendant qu'elle fait toumer le moulin, l'autre lui fournit le mais en le jetant par poignées dans le trou de la meule. Le maïs sort de là plutòt concassé que moulu; on le vanne en le faisant santer sur un gyand plat sans bord, qui a environ 8 pouces de diametre. La . 


\section{( 550$)$}

farine se donne à la volaille; on le réduit par la meule en parties plus ou moins ténues, suivant le désir des personnes qui en font usage.

Lorsqu'il est destiné à la nourriture des noirs, il n'est concassé qu'en trois ou quatre morceaux, et porte le nom de gros-balle lorsqu'il est cuit.

Pour le faire cuire, on se sert de grandes nıar mites en fonte, élevées sur trois pieds. On lave le maïs ainsi concassé, de manière à enlever toute la farine; on le met dans le vase en y ajoutant une quantité égale d'eau. On fait bonillir jusqu'à ce que le mélange ait pris la consistance pàteuse, en ayant soin de rejeter l'écume qui contient encore quelques matières étrangères, et on met du sel pour augmenter la saveur. Le maïs est cuit au bout d'une heure on une heure et demie. Alors on mêle des haricots blancs cuits à part, ou des lentilles qui auront dủ être cuites avec le maïs; on met. un peu d'oignon légèrement frit, du poivre, du beurre, ou mieux du sain-doux, et on sert.

Lorsque le maïs est moulı plus fin, il prend le nom de maïs fin; il s'emploie pour la nourriture des nẹgresses qui nourrissent leurs 
enfans et de jenues négrillons: on le fait enire long-temps de la mème manière que nous venons d'indiquer, et la bouillie qui en résulte ici se nomme cange.

Ce mème mais étant cuit, presque jusqu’à siccité, est servi sur les tables des créoles, surtout à déjeùner'; il est appelé mä̌s sec: il sert de pain. Il se forme ordinairement une cronte plus dessćchće, de couleur brunàtre, antour de la marmite; elle se nomme sampangue : on la sert avec le maïs.

Les jeunesépis seconfisent au vinaiggre, etentrent quelquefois dans la confection des achars.

Le grain de maïs sert aussí à Bourbon pour la volaille.

Je puis attester, par expérience, la bonté de ce mets, appelé maïs sec; car j'en ai mangé plusieurs fois ici à Paris, chez une créole de Bourbon, qui le faisait préparer par un noir qui était ì son service comme cuisinier, et qui était vent avec elle en France. On le sert sur les tables pour les déjeùners en mème temps que la viande, $\mathrm{et}$ on en met une portion sur son assiette avec une portion de viande : il sert alors de pain, qui ne parail pas sur ces tables. 
On prend alternativement de ce maïs et de la viande, comme chez nous on prend du pain et de la viande : ce mets est trés nourrissant, facile à digérer; il en faut peu pour rassasier.

Effets de la nourriture du maïs.

Les effets du maïs, considéré comme aliment, sont nécessairement variables, suivant que l'on prend cette nourriture seule ou mêlée à d'autres substances végétales ou animales, suivant qu'elle sera prise par des hommes sains et vigoureux, ou par de jeunes enfans dont les organes digestifs, encore dans leur enfance pour ainsi dire, ont besoin d'une nourriture qui leur soit directement appropriée, ou encore par des femmes qui allaitent et ne peuvent pas être alors regardées comme dans un état de parfaite santẻ, car elles doivent surveiller leur régime, non seulement pour elles, mais aussi pour leurs nourrissons.

Le maïs, avant d'être traité sous ces différens points de vue, doit être examiné sous celui de ses principes constituans. Ainsi, l'analyse nous fait voir que le maïs est presqu'entiè- 


\section{( 533$)$}

rement composé d'amidon, de matières suerées et mucilagineuses; lit fécule y est en assez grande quantité; quant au gluten, s'il y existe, il y joue $n$ rôle très minime.

Le maïs n'agira donc que comme tous les régétaux qui contiennent du sucre et de la fécule, èest à dire que son action, quoique ríparatrice, agyira aussi comme rafraichissante et émolliente.

Prise seule, telle sera l'effet de cette nourriture.

Associée à d'autres régétaux, elle pourra, suivant leur nature, devenir plus réparatrice et même fortement stimulante : tel serait, je crois, l'effet du macaroni au maïs et de la polenta des riches Italiens. Son action changera encore si, au lieu d'eau, on prend du vin : et que de nuances on pourrait encore obtenir, suirant la qualité et la quantité de ce vin! Enfin le maïs, joint à des substances animales, donnera nécessairement une action beancoup plus réparatrice. 


\section{(334)}

Chez l'homme.

Le maïs seul peut nourrir, à des doses très petites : ainsi, j'ai fait l'expérience de me nourrir, à plusieurs reprises, pendant deux et trois jours consécutifs, de gaudes cuites à leau. La quantité prise dans un jour n'a jamais dépassé ı 90 à $205 \mathrm{gr}$.; le terme le plus ordinaire a été de 200 grammes; un quart de beurre a toujours suffi pour les trois bouillies qui composaient ma nourriture d'un jour, l'eau seule était mon unique boisson.

La quantité de mes urines auggmentait sensiblement : ainsi, dans vingt-quatre heures, j'en rendais I, 280 à r, 3oo grammes, jamais plus ni moins; mes selles étaient plus libres, mes forces égales; point de changement dans le sommeil; mon pouls, quatre heures après le repas, donnait cinquante-huit pulsations par minute.

Lorsqu'au contraire je prenais ma nourriture ordinaire composée de substances végétales et animales, et pour boisson du vinblanc, je n'avais uriné, au bout de vingt-quatre heu- 


\section{( 535$)$}

res, que 1, r6o ì 1, 170 grammes de liquide; mes selles citaicut beancoup plus dures, surtout si quelques jours auparavant je m'étais nourri de maïs; mon sommeil était le même, et mon pouls, tonjours quatre heures après le repas, domait soixante-deux pulsations.

Plusieurs médecins ont déjà parlé de l'action du maïs sur la sécrétion mrinaire; mais je crois ètre le seul qui, jusqu’à présent, me sois donné la peine de produire des résultats posilifs.

Quant à l'eflet laxatif de cette nourriture, elle ne peut plus, du moins par rapport à moi, être mise en doute; toutes les fois que, par des circonstances extraordinaires, je suis resté (pendant le temps de ces expériences, trois mois) sans prendre du maïs, sous une forme ou sous une autre, mon ćtat de constipation habituel n'a pas manqué de se reproduire.

On a expérimenté qu'une bouillic composéc de 48 livres de maïs, 168 livres d'ean et 2 livres de sel, a nourri et rassasié cent dix persomnes à Perpignan. Cette bonillie leur a été distribuée en deux repas. 


\section{( 336$)$}

Le docteur Venel dit (Encyclopédie, t. VI, feuille I 756, art. Farine et Farinenx) qu'il ne faut pas 6 onces de riz ou de farine de riz et de maïs pour vivre pendant une journée entière, et être en état de faire certain exercice. D'après Varennes de Fenille (I), cette nourriture est saine, et quoiqu'assez lourde en apparence, elle est de facile digestion, même pour les estomacs débiles; mais c'est aussi pour cette raison qu'elle ne serait pas suffisante pour soutenir les gens de la campagne, pendant la saison des travaux.

On pourrait facilement trouver des preuves pour contredire la dernière opinion de Varennes; aussi Burger (2) dit : Les Nègres condamnés aux travaux pénibles des sucreries, les porte-faix italiens, les scieurs de bois. italiens, qui n'ont d'autre nourriture que le maïs, se sont convaincus, par une longue expérience, que rien ne soutient mieux les forces que la bouillie de maïs.

(1) Observations, cxpériences et mémoires sur l'agriculture, p. 69 , in-8․ Lyon, I 779 .

(2) Ouvrage cité (en allemand), p. 5 r 7 . 


\section{$(577)$}

C'est, selon Parmentier (payge 2/73), la subsistance principate de lous les gens de la campagne pendant l'hiver en Bompogne el en Franche-Comté : e'est le premier repas des batteurs en grange el des domestiques; les gandes sont en si grand honneur parmi eux, qu'une de leurs conditions, arant d'entrer en service, éest qu'on leur donnera des gुandes, el ee serait pour eux un malheur réel d'en être privés. Ce dejenoner est preféré à tout antre; il les soutient une partie de la journée. Chez quelques peuplades du Brésil, au lieu d'argent pour prix du travail qu'ils ont fait, on leur donne du maïs qui leur sert de principale nourriture.

Si la farine de mais était employće dans diverses proportious pour le pain de munition, et aussi pour le pain et les différens mets que I'on distribue dans les hôpitanx, dans les maisons de détention, les bagnes, les dépôts de mendicité, pour distribuer aux indigens, et pour la confection des alimens de la classe ouvrière, sa consommation deviendrait considérable, et tout en ouvrant une nouvelle lnanche d'industric aux agricultems, elle pro- 
curerait des avantages immenses et une éconounie véritable au Gouvernement.

D'après ce que nous avons dit plus haut de son emploi, on voit qu'il pourrait suppléer quelquefois ou être mélangé à d'autres gुrains, ainsi au froment, dont la quantité consommée i Paris pour la nourriture de ses habitans, au nombre de huit cent soixante-quinze mille, est presqu'incroyable.

La consommation journalière de Paris, pour ${ }_{1} \$_{2} 6$, était de quinze cents sacs, du poids de 320 livres chaque, donnant un total de 624, ooo livres de pain mangées journellement. Quand le blé est à un prix moyen, le total pour l'annće est de 227,760 , 000 livres de pain.

Des résultats d'expérience ont prouvé que, dans les temps de disette, la pomme de terre, pour suppléer au pain, doit être employée dans une quantité triple. 3 livres de pommes de terre nourrissent autant qu'une livre de pain.

Je m'en rapporte ici aux tableaux statistiques sur la ville de Paris et le département de la Seine rénnis d'après les ordres de M. le comte de Chabrol, préfet de la Seine, i 829 , in- $4^{\circ}$. 
(lideherches comernant la dípense qui est faite anmuellement à Paris, ponur la nonrriture des halitans.)

Quant anx ressources qu'il pourait offrir pour les étahlissemens publies, je ne citerai qu'un seul fait déjà bien comm ; il est citc par M. Lelière.

Aux Éats-Unis, les quakers qui administrent les maisons de force où l'on détient les criminels condamnés pour toute leur vie, les nourrissent avee de la farine de maïs bouillie à l'eau aree de la mélasse. Ces eriminels, s’ils se conduisent bien, peuvent être rendus à la société, ce qui arrive assez sourent, et l'on n'a jamais eu d'exemple qu'un de ces hommes réhabilités ait été repriș une seconde fois de justice. Les directenrs de ces établissemens sont assez modestes pour attribuer une partie de leurs succès à cette nourriture, dont ils regardent l'usage comme calmant et adoucissant; l'un d'eux m'assurait qu'il la croyait propre à adoucir et à changer les caractères portés à la violence.

Cette expérience heureuse et journalière faite dans toute l'étendue d'un grand pays par 
des administrateurs attentifs, sages, doux, vigilans, bienfaisans, est une forte présomption en faveur de leur théorie, qui est le résultat d'observations raisonnées. Elle est conforme à la vérité reconnue de l'influence des peuples sur leurs humeurs, leurs habitudes et leurs caractères ( 1 ).

Il n'est pas indifférent de rapporter à ce sujet l'extrait d'une lettre écrite à M. Bossange, en date du 24 novembre r 829, par M. d'Haussez, alors ministre de la Marine et des Colonies, ancien préfet des Landes; cette lettre m'a été communiquée.

"Je m'empresse, Monsieur, de v́ous transmettre les renseignemens que vous m'avez demandés relativement à l'influence de la substitution'du maïs aux diverses variétés de millet employées long - temps dans le département des Landes, comme base principale de la nourriture des habitans des campagnes.

(1) Essais sur la culture du maïs, etc., p. 37, 38, 41, in-12. Paris, Didot, 1827 . 


\section{$(5 / 1)$}

"Ces renseignemens résultent des observations que jai recueillies ou laites pendaut les opérations du recrutement dont j’étais chargé.

"Le département est divisé par l'Allour en deux parties très distinctes, la Chalosse et les Landes. La Chalosse, situce sur la rive gauche, est un pays montucux, coupé par des vallées profondes, mais bien ouvertes et bien cultivées. Sa population présente le phénomène d'une amélioration physique dont on peut suive la gradation en comparant les vieillards aux hommes de vingt ans. Les uns sont petits, maigres, rachitiques, tandis que ceuxci sont d'une taille élevée, de formes très prononcées et d'un caractere énergique. L'intervalle qui sépare les deux àges offre une espèce d'hommes qui tend vers un perfectionnement dont on peut expliquer les progrès par ceux qu'a faits l'introduction du maïs dans la nourriture du peuple. Ce n'est que ver's le milieu du ternier siecle, que la culture de eetu plante préciense a ité introduite dans lo Béarn. La race quielle a trouve formée a, diton, gagué en longéviti, mais non en déve- 


\section{$(342)$}

loppement; celle à la nourriture de laquelle, depuis l'enfance, elle a été employée, fournit la preuve de ses avantages, et c'est surtout à la seconde génération que les effets en sont le plus sensibles.

"On peut suivre cette observation sur la rive droite de l'Adour. Le maïs, cultivé sur le littoral du fleuve, a produit des résultats semblables à ceux qui se font remarquer sur la rive gauche. Dans l'intérieur des Landes, une comparaison fàcheuse s'établit entre les habitans des communes où il n'est pas cultivé et ceux des localités qui s'en nourrissent. La cause de la différence ne peut être l'oljet d'un doute, lorsque toutes conditions égales d'ailleurs, relativement à la situation des habitations, à la nature des eaux, aux habitudes dé travail, on remarque que le peu de développement des formes et de durée de la vie apparlient aux communes où l'on ne récolte que le millet, tandis que les avantages contraires sont assurés à celles où la culture du maïs esı gुénéralisée.

"Dans les premières, la durée moyenne de la vie est de vingt-deux à vingt-trois ans. 


\section{( 5.5$)$}

"Daus les dermieres, elle s'ileve jusqüä trente ef un alls.

" Recere\%, ric., atc.

\section{" Le Ministre de la marine al des colonies.}

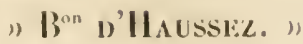

M. Ie comte de Chabrol dit que l'on a remarque que depuis que cette culture s'est propagée daus les deux arrondissemeus de Ceva et d'Aequi, la population s'est accrue, el que l'émigration qui avait toujours lieu à la suite de la famine dans les années de gyrèle, avait singulièrenent diminué (1).

\section{Chez les enfans en bas cige.}

Quoique j’aie répété mes expériences sur un grand nombre de jeunes enfans, pour être moins long, je n'en citerai que deux : le sujer de la première est ma nièce, àgée de

(1) Sintistique du dipartement de Montenoltc, t. II, p. 139 . 


\section{( 344$)$}

deux ans, et l'autre, l'enfant de $\mathbf{M}^{\text {me }}$ Bégin, àgé de huit mois, dont il sera question plus tard. Au reste, tous les enfans élevés en Bourgogne, dans le midi de la France, en Italie, ont-ils d'autre nourriture?

Les mets que l'on prépare avec le maïs, et dans la composition desquels il n'entre pas d'épice ni d'aromate, sont aussi ceux qui conviennent et qui plaisent davantage aux enfans; la bouillie surtout leur paraît très agréable. Ils aiment aussi beaucoup les différentes espèces de pain et de gàteaux que l'on prépare avec ce grain : chez eux aussi, la quantité d'urine auggmente. Il m’a parn qu'ils étaient plus gais après avoir pris cette nourriture.

En effet, peut-être par sa simplicité et par l'absence de matière fermentescible, convientelle mieux à leur faible estomac que les préparations de froment, par exemple; sa composition pourrait rendre raison de cette observation. 


\section{$(3,5)$ \\ Chez les femmes qui allaitent.}

II est assez diflicile a Paris de tronver des femmes qui veuillent se prèter à quelqu'expérience qui gène leurs habitudes. Voici cependant une observation complète dont le sujet est $\mathbf{I}^{\mathrm{me}}$ Bégin (Catherine Leroux), rue des Marais-Saint-Martin, $1^{\circ} 27$, iggée de trente ans. Il est vrai de dire que cette fermme est née et a áté élevée en Bourgogne, et que, depuis longtemps, elle était déjà faite à cette nourriture particulière.

Pendant trois jours, elle s'est nourrie avec a livres et demie de farine de maïs cuite i l'eau avec dn beurre, ne buvant que de l'eau; cette seule nourriture a pu lui suffire, et son lait est venu plus abondamment. Du reste, point de changement dans son état de samté habituel ni dans ses antres sécrélions; mais cette femme se nourrit frequemment de cette bouillic.

Cette axpérince a eté plusienrs fors répété par elle jusqu à l'ipoque du sevrage, el tonjours e'l obternant les mèmes résultats. Sal sante 
a toujours été bonne et son notnrisson est venu parfaitement bien; l'enfant a été ensuite nourri avec cette farine, il est actuellement très bien portant.

Une nourrice du Port-Marly, près de SaintGermain, a bien voulu aussi tenter quelques essais ; mais les expériences ont été mal faites.

$\mathbf{M}^{\mathrm{me}}$ Martin, rue du Cherche-Midi, $\mathrm{n}^{\circ}{ }_{2}$ ।, qui n'en avait jamais mangé, en a été inconımodée; mais depuis une quinzaine de jours, elle était souffrante.

D'après des renseignemens bien positifs et pris seulement à ce sujet, on m'a assuré que toutes les femmes de la Bourgogne contimuaient cette nourriture lorsqu'elles nourrissaient ( 1 ).

(1) Parmi les exemples que j'ai recueillis, six femmes ont vécu elles-mêmes et ont alimenté leurs nourrissons selon les usages du pays (Landes), mais saus maïs pourtant; six autres ont vécu et ont alimenté, avec du maïs seul ou presque seul et leur lait, des nourrissons de six mois. Toutes choses égales, et cn tenant compte de l'àge, de la constitution, de la situation locale, de l'habitation a la campagne ou à la ville, de l'histoire de leurs précédens allaite- 


\section{$(347)$}

A l'époque du concour's, je n'avais pu suire entièrement que l'observation de $\mathbf{M}^{\text {me }}$ Béggin ; depuis j’ai en plusieurs fois l'oceasion de ripétér ces expériences, et jén ai toujours été très satisfait.

Parmi tous les mets et hoissons que l'ou peut preparer avec le mais, tous cenx que j’ai désiģnés ne point être lourds ni aromatisés, et pour les boissons, celles qui ne sont point alcoolisćes, peuvent convenir aux femmes qui allaitent et entrer an moins en partic dans leur nourriture habituelle.

mens, etc., celles qui ont mis le mais en usage ont mieux conservé leur santé, leur embonpoint el leurs forces; les nourrissons, de leur côté, ont été plus forts, plus beaux, plus sains que ceux des autres (Mimoire communiqué de M. Lespes.)

HIN. 
L'Auteur recevra avec reconnaissance les renseignemens qui lui seraient adressés franco, rue d'Assas, $\mathbf{n}^{0} 7$, à Paris. 


\title{
TABLE DES AUTEURS
}

\author{
L:T IES TITRES W'OUVRACES
}

CITES DANS CE TRAITE.

Aglo (Augustine). Antiquities of Mexico, ete. London, 1830.

Amoreux, D.-M. Mémoire sur les haies, couronné par l'Académie des sciences, belles-lettres et arts de Lyon, dans la séance publique du 3 août I 84 . In-I 2.

Annales de l'Agriculture française, rédigées par Tessier. Paris, in-8, $\mathbf{M}^{\text {we }}$ Huzard.

Auguste de Saint-Hilaire. Foyage dans Iintérieur du Brésil. Paris, 1830, 2 vol. in-8.

Bastien. Nouvelle Maison rustique. Paris, r $79^{8}$, in-4, tome $\mathbf{I}^{\mathrm{er}}$.

Baudouin (J.). Histoire des Incas; traduite de l'espagnol de l'inea Garcillasso de la Vega. Amsterdam, 1704, in-12, tomes I et II.

Bégulliet. Traité de la connaissance générale des 
grains et de la monture par économie. Paris, $177^{5}$, in-8.

Bibliothèque physico-économique. Parıs, I8 9 , tome V.

Blachw. Herbarii Blackvelliani auctorium collectio stirpium, etc. Norimbergæ, г 773.

Bonnet (Charles). Recherches sur l'usage des feuilles dans les plantes. Gœutingue et Leyde, 1754 , in- 4 .

Burger (D. Jean). Traité complet sur lhistoire naturelle, la culture et l'emploi du maïs ou blé de Turquie (en allemand). Vienne, I $\$ 09$, in- 8 .

Cadet de Vaux. Moyen de prévenir le retour des disettes. Paris, 1812 , in-8.

Cauxors (Robert-Regnault). Histoire naturelle et morale des Indes, tant orientales quoccidentales, par Acosta; traduite en français par... Paris, 1600 , in-8.

Chabrol de Volvic (comte de). Recherches statistiques sur la ville.de Paris. I 829, in-4.

Le même. Statistique de l'ancien département de Montenotte. Paris, 1824,2 vol. in -4 .

Challes Estienne. L'A griculture el Maison rustique de Charles Estienne et Jean Liẻbault. Paris, 1640 , in-4. 
Coвветт (William). Ouvag" sur le mas (en anglais). Londeres, in-12.

Counet ne luleneure. Le pelit Magasiu cionomique. letil in-8.

1) ampiente Guillaume). Fogage autourdumonde. Ronc11, 715, 5 rol. in-12.

1)esbury. Némoire sur la meilleure maniere de tirer pari des landes de Bordeanx, etc., qui a remporti, en $17 z^{6}$, le prix propose par l'Aeadémie roale des belles-lettres. sciences et arts de Bordenux. Bordeaux, 1776, in-4.

l'escription de l'Ég)ple, édit. Panckouche. In-s, tome XIX. Flore de Delile.

Desplaces. IIstoire de l'Agriculture ancienne. extraite de l'Histoire naturelle de Pline.

l)ictionnaire de l'Industrie, par une Société do gens de leures. Paris, r795, in-8, tome $\mathbf{V}$.

Jictionnaire d' Histoire naturelle. Edit. Déterville, r8o3, art. Maïs, in-8, tome XIII.

Dictionnaire d' Histoire naturelle (Nouvenu). Paris, Déterville, r 807 , in-8, 1 . XVIII.

Diclionnaire des Sciences naturelles. Pitris, I.crrault, 1823 , in-8, t. XXVIII.

Dictionnaire des Sciences médicales. Panckoncke, in-8. 


\section{( 352$)$}

Dictionnaire de médecine, en 18 vol. In-8, tome XIII.

D’O. D'Apper. Description de l'Africue; traduite du flamand par... Amsterdan, i680, in-fol.

Dransy. Mémoire sur les avantages que le roy aume peut retirer de ses grains. In-4.

Encyclopédie. Deuxième édition, tone XX.

Encyclopédie. 1756, infol., tome V.

- Fabroni. Instruzioni elementari di agricoltora. Turin, I $79 \mathbf{r}$.

Fauconpret (DE). Histoire de la vie et des voyages de Christophe Colomb, par Weshington Irwing. 1828 , in-8.

Fenille du Cultivateur, année 5-6. Paris, an vı de la république, tome VII.

Gallo (Agost). Le venti giornate dell' agricoltora. Deuxième édition, 1775 .

Godard. Histoire naturelle des Lépidoptères, continuée par Duponchel. 1827, 1. IV.

Gothard (Joh. Christian). Die cultur und benubung dis turcischens maizens oder mays (en allemand). Erfurt, I 797 , in-1 2.

Harasti di Buda. Della collivazione del maiz. Vicenza, 1788 , in-8.

Humboldт (DE). Voyage aux régions équinoxiales. I 822 , in- 8 . 


\section{( 555$)$}

Humbolot (ne). Fissai politique sur le rou aume de la Nouvelle-Tispagne. In-8.

Imuofy (Franc.-Jacob). Zea ma) dis morbus ad ustilaginem vulgo relatus, 1784. Argentorati, in-fol., fig.

Instruction sur les usages et la culture du Blé de Turquie comme grain. Publiéc à Paris en 1786. Instruction sur les usagres el la culture du Maïs, publiée à Paris par ordre du Ministre de I'Intérieur. Germinal an iv, brochure de 32. pages.

Instruction sur la culture du Mä̈s ou Blé de Turquicel les divers usages de cette plante, avec un Progranme du concours ouvert pour sa culture, publiée par la Société d'Horticulıure de Paris. 1828 , in- 8 , brochure de 1 i pages.

Instruction sur la culture du Maïs, avec un Programme du prix fondé par $\boldsymbol{M}$. Bossange père pour la meilleure culture du Maïs, publiée par la Société d'Horticulture de Paris. 183o, in-8, brochure de 34 pages.

Journal de Pharmacie, t. VII, 1821.

LaEt (DE). Novus orbis seu descriptionis Indice occidenealis. Ludg-Bat., 1633, in-fol.

I.nmane. Le Cultivateur anglais ou OEuvres 'hoisies d' Agriculture at d'Economic rurale ot 
domestique, traduit de l'anglais. Paris, 1801 , 18 vol. int 8 .

La Rochette. Histoire de l'Amérique de Robertson. 1827 , tome $\mathbf{I}^{\text {er }}$.

Lelievir de Ville-sur-Arce. Essai sur la culture du Naïs et de la Patate douce. Paris, Didot. ${ }_{1827}$, in- 12 .

Lespez. Essai sur le Maïs ou Blé de Tiurquie, considéré sous ses rapports lyg giénique et medical. Paris, 1825 , thèse in- $4, n^{\circ} 99$.

Liébault (Jean). Voyez Charl.es Estienne.

Marabelli. De Zea mays planta analytica disquisitio. Pavie, $179^{3}$, in-8.

Marca (Pierre de). Histoire du Béarn. Paris, I640, in-fol.

Martyr. Extrait des recueils des iles trouvées en la grande mer Océanne. Paris, r532, in-4.

Mémoire de la Société d' Agriculture du département de la Seine. In-8.

Mémoire de la Société d'A griculture du département de Seine-et-Oise. In-8.

Mémoires de la Société d'Agriculture de Turin. 1812 , in 8 , t. IX.

Mrr.er. Dictionnaire des Jardiniers. Paris, 1785 , in $-4,1$. VIII. 


\section{( 355$)$}

Montesqueu. Espril des Lois. Fidition de Dalibon, $18 \% 7$, in-8, tome III.

Monson. Ilist. plant. ed. lat. i g r 5, in-fol.

Panmentier. Le Mä̈s ou Blé de Turquie apprécié sous lous les rapports, mémoire couronné le 25 aoni r 784 par l'Académic de Bordeaux: Paris, 1812, in-8.

Parmentren. Trailé théorique el pratique sur la culture des grains. Paris, 1802 , in-8.

Panmentifr. Le parfuit Boulanger, ou Trailé complet sur la fabrication el le commerce du pain. Paris, 1778 , in-8.

PALW (DE). Recherches philosopliques sur les Américains. 1774, 2 vol. in-12.

Pluguer. Cours d'agriculture pratique. I 809 , in -8 , tome Jer.

Polem (Jean). Histoire naturelle el générale des Indes. Paris, i556, in-fol.

Prévost. Hisloire générale des Yoy ages. La Haye, 1747 , t. IV.

Relations véritables el curicuses de l'ule de Madagascar el du Brésil. Paris, 1651, in-4.

Revue des deux Mondes, III' année. Janvier et février 1831 .

Ricinard. Histoire naturelle médicale. 1831 , 2 vol. in-8. 


\section{( 356 )}

Roulox Baro. Voyage inséré dans un autre ouvrage. Voyez Relations véritables, etc., p. 97.

Rozier. Cours complet d'Agriculture ou Dictionnaire universel d'Agriculture. Paris, 1785 , in-4, tome VI.

Rovgier de la Bergerie. Cour's d'Agriculture ou l'Agronome français, par une Société d'agronomes, dirigé par... 1819, in-8, tome I ${ }^{\text {er }}$.

Sahagun (Bernardino De). Historia universal de las cosas de Nueva Espana.

Seringe. Monographie des céréales de la Suisse. Berne, 1818 , in-8.

Simonde. Tableau de l'Agriculture toscane. Genève, i 801 .

Tillet. Dissertation sur la cause qui corrompt et noircit les grains de blé dans les épis, et sur les moyens de prévenir ces accidens. Bordeaux, 1755,2 vol. in 4 .

Thaer. Principaux instrumens d'agriculture. Paris, 1821 , in-4.

Thevenot. Recueil de voyages. Découverte de l'Amérique septentrionale. Paris, 1787 , in-8. Tollard ainné. Traité des végétaux gui composent l'agriculture de l'Empire français. Paris, I 805 , in-1 2 . 
TunNias helibal.

Young (Arthur). Voyages en France pendant les anncés $1787,1788,1789$ et $179^{\circ}$; traduits de l'anglais par F'. S. Paris, 1794 , in-8, deuxieme édition.

Zaxon. Dell'agricoltora, dell' arti elettere. $1765-$ 1767 . Tome V, lett. 15.

Zarate. Histoire de la découverle el de la conquéte du Pérou, traduite de l'espagnol de... par S. D. C. Amsterdam, i goo.

Wanden. Riecherches sur les antiquités de $l ' A$ mérique septentrionale. Paris, 1827 , in-4.

Willmet. Pliytographie encyclopédique ou Flore économique. Paris, I808, tome III. Ulloa (don). Mémoires philosophiques, historiques, physiques, concernant la découverte de l'Amérique. Paris, 1782,2 vol. in-8. 


\section{ERRATA.}

Pağe 53, ligne 2. Lisez : aliquâ sacchari comnunis.

Page 53, ligne 5. Paritate; lisez: puritate.

Page 93. La note de M. de Grégory à reporter après ces mots, XVe siècle, p. 94 . 


\section{EXPLICATION DES PLANCHES.}

\section{PI.INCIII: I}

Fig. 1. Aspect grénéral, en raccourci, du mais amivé a son plus grand développement.

Fig. 2. Épide mais mutr, beaucoup plus petit.

Fig. 3. Naissance du mais, huit jours de plantatios, grandeur ordinaire.

Fig. 4. Grosseur moyeme d'un grain de mais parvenu à maturité.

\section{P.ANCHE; 11}

MaChINE EN FONTE POUR ÉgRENER LE Maís.

Fig. 1. La machinc vuc de: profil.

A, Manivelle à bras.

B, Arbre du mouvement.

CCC, Grenouilles a boite de l'arbre du mouvement.

D), Montant à chapeau de la machine.

E, Régulateur. 


\section{$(360)$}

F, Va-et-vient.

G, Arbre de la grande roue perpendiculaire.

II, Petite roue d'engrenage.

I, Grande roue d'engrenage.

KK, Patin en bois de la machine.

L, Roue taillée ì dents de râpe.

SS, Boulons pour fixer la machine sur son patin.

Fic. 2, La machine vue de face.

B, Arbre.

C, Grenouille.

D, Montant à chapeau de la machine.

E, Régulateur.

KK, Patin en hois.

L, Rone taillée à dents de râpe.

M, Entonnoir pour recevoir l'épi.

P, Épi.

R, Maïs égreué.

SS, Boulons pour fixer la machine sur son patin 
. 


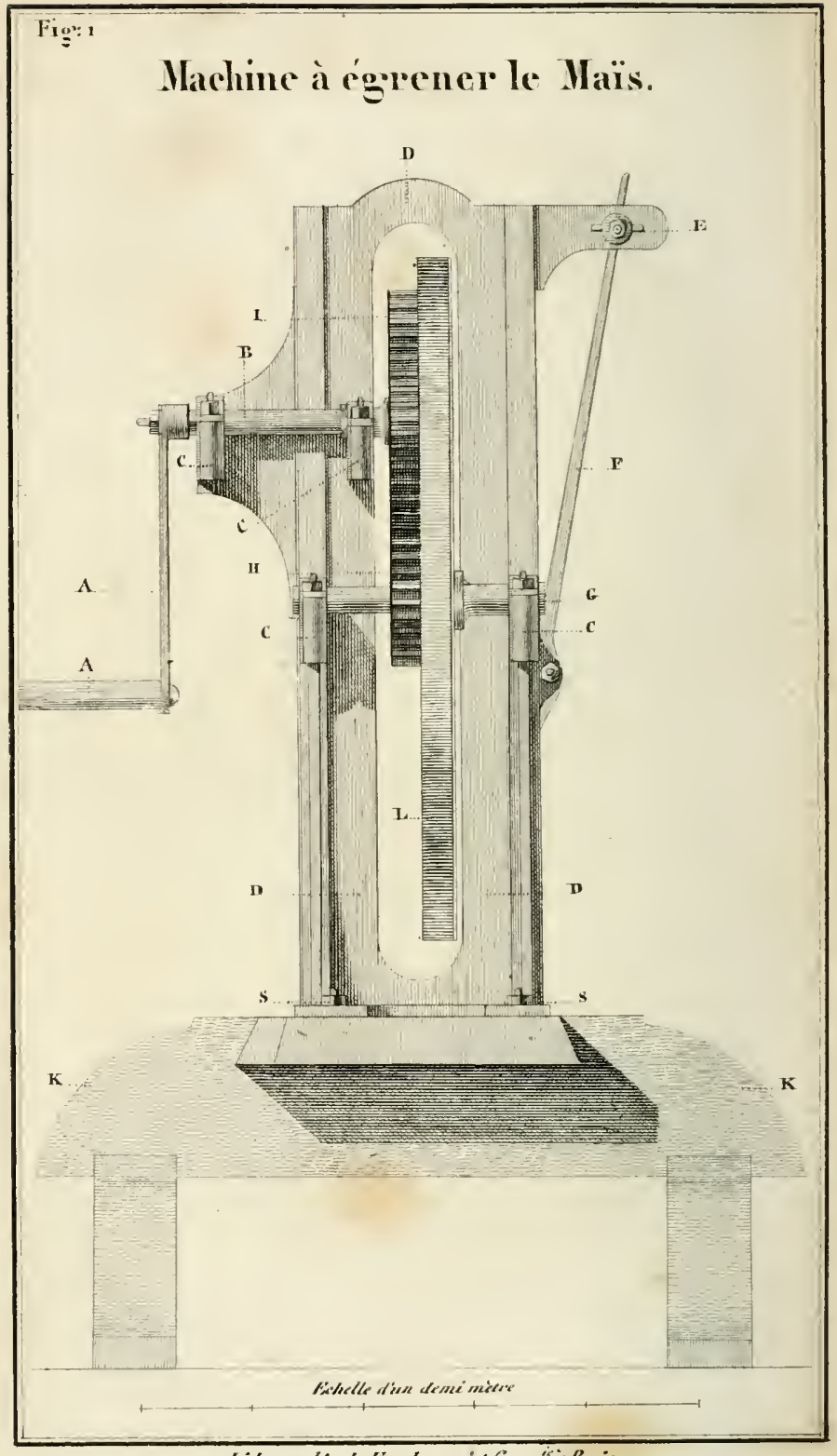

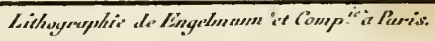




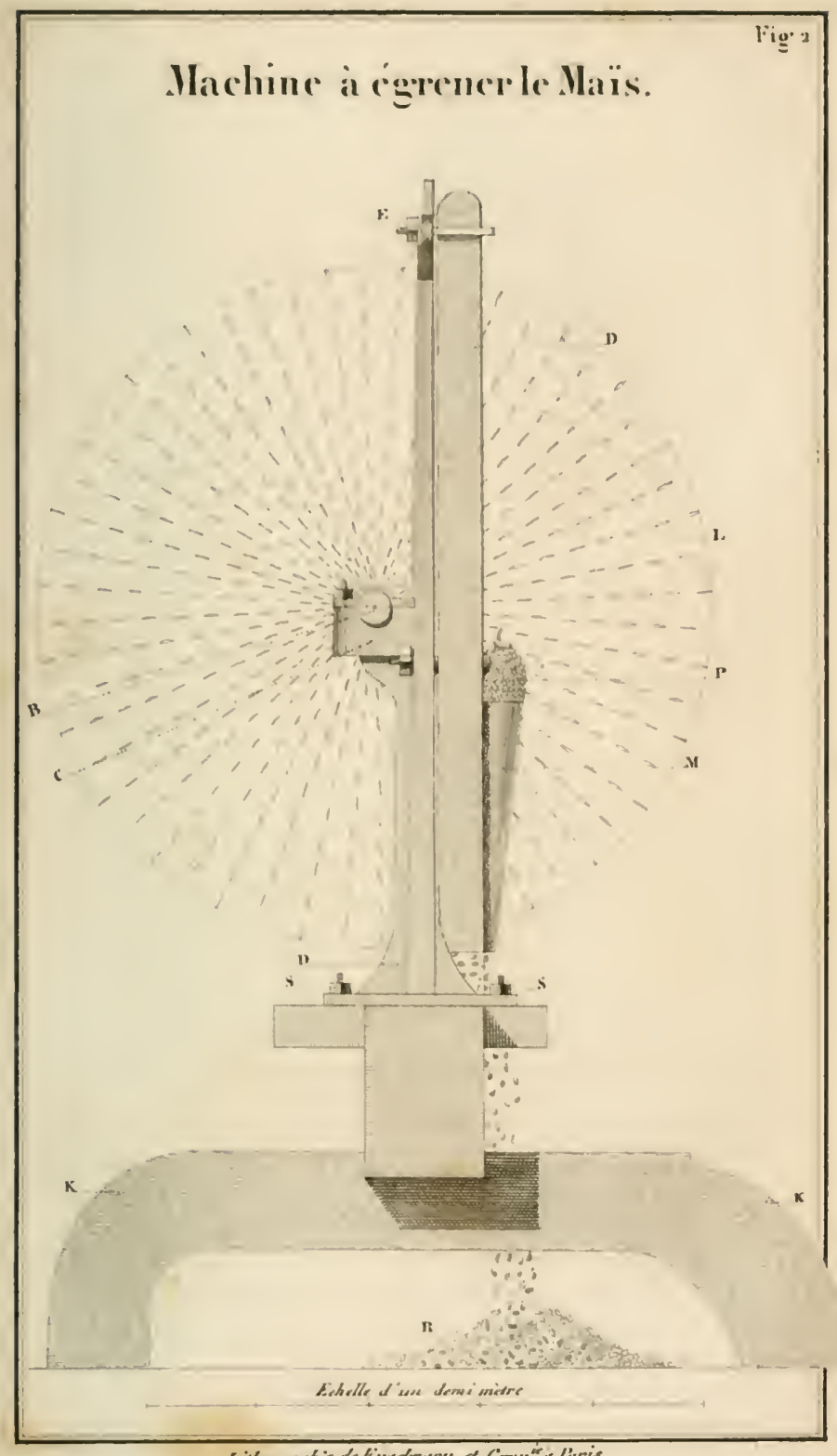

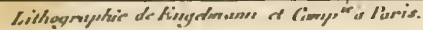





\section{TABLE DES NATIERES.}

Pages

Dédicack. . . . . . . . . . . . . . 5

AvaNT-PROPOS. . . . . . . . . . . 7

Historight. . . . . . . . . . . . 15

le mais était-il comnu avant la décomverte de

l'Amérique? . . . . . . . . . . . . 23

Noms vulgaires du mais. . . . . . . . . 3 3

Origrine du nom de blé de Turquie. . . . . . 34

Description hotanique. . . . . . . . . . 35

Description du \{ros matis. . . . . . . . 37

Variétés. . . . . . . . . . . 3 3

Analyse. . . . . . . . . . . . . 49

Des accidens du mais. . . . . . . . . . . 56

Maladies du ınas. . . . . . . . . . . 59

Remède de ces maladies. . . . . . . . . . . 69

Des animaux qui allaquent le mais. . . . . . I6.

Culure. . . . . . . . . . . . . 77

Des terres propres an mas. . . . . . . . . 78

Introduction de sa culture dans differens pays. . $y^{3}$

Te sa place dans len assolimens. . . . . . 97 
Engrais. . . . . . . . . . . 101

Préparation du terrain. . . . . . . . 107

Des semailles. . . . . . . . . . . . . . 109

Choix de la semence. . . . . . . . 112

Préservatifs de la semence. . . . . . . . . . . 115

De l'ensemencement. . . . . . . . . . I I 6

$1^{\circ}$. En suivant la charrue. . . . . . . 117

$2^{\circ}$. Sillons en travers. . . . . . . . 118

3․ A la volée. . . . . . . . . . . 120

$4^{\circ}$. A la houe.............. . I6.

$5^{\circ}$. Au plantoir, au moyen du cordeau. . . . 124

$6^{\circ}$. Avec le semoir à brouette. . . . . . . 123

$7^{\circ}$. Machine à semer de J. Burger. . . . I Ib.

Culture intermédiaire. . . . . . . . . . 129

Utilité du maïs dans les jardins. . . . . . . . 133

Culture ordinaire. . . . . . . . . . . . 134

Élaggage et étètement du maïs.. . . . . . . 145

Culture comparée. . . . . . . . . . . . 148

Récolte. . . . . . . . . . . 152

Rapport. . . . . . . . . . . 154

Commerce du maïs. . . . . . . . . . . . . 160

Conservation du maïs en épi. . . . . . . . . . . 164

Dessiccation du maïs par l'intermède de l'air. . . 165

Maïs suspendu au plancher. . . . . . . . . I

Maïs répandu dans le grenier. . . . . . . . . 166

Séchoirs à maïs. . . . . . . . . . . . Ib.

Séchoir de M. de Méja. . . . . . . . . . . . 168

Séchoir de M. Lelieur. . . . . . . . 171 
Séchoir de M. Mathieu de Dombasle..... . 172

Mais séché au soleil. . . . . . . . . 174

Maïs séché à l'étuve. . . . . . . . . . Ib.

Mais séchí au four. . . . . . . . . 175

Mais séclué à la fumée. ......... 177

Conservation du mais en grain. . . . . . . 179

Egrenage des épis. . . . . . . . . . . 180

10. la main. . . . . . . . . . . 181

$2^{\circ}$. Par le frottentent. . . . . . . . . Ib.

$3^{\circ}$. Avec une faucille. . . . . . . . . 182

$4^{\circ}$. Sur uu tombereau.. . . . . . . . . . Ib.

$5^{\circ}$. Avec le fléau. . . . . . . . . . 184

$6^{\circ}$. Sur une barre de ler.......... Ib.

$7^{\circ}$. Sur une nesure. . . . . . . . 185

8. Avec la filière. . . . . . . . . . . Ib.

$9^{\circ}$. Avec la machine de M. Fol. . . . . . 186

Mais en tas. . . . . . . . . . . . 187

Maïs en sacs isolés. . . . . . . . . 190

Maïs dans des fosses ou silos. . . . . . . 191

Divers autres moyens de conserration. . . . . $19^{5}$

Mouture des grains. . . . . . . . . . . 198

Ecrasement des grairs. . . . . . . . 199

Farine de maïs en sacs isolés. . . . . . . 201

Emplo1. - Nourriture des animanx avec lo mais. 203

Usages économiques des tiges. . . . . . . . . 214

Feuilles. . . . . . . . . . . 2 . . . 5

Spatlic. . . . . . . . . . . . . Ib.

Riafle. ........... 217 
Son.............. 217

Farine. . . . . . . . . . . . 218

Euptor en médecine. - Usage du mais comme médicament. . . . . . . . . . . . 219

Du maïs pour la nouriture de l'homme, des

enfans en has âge et des femmes qui allaitent. 23 I

Tiges. - Sucre. . . . . . . . . . . 232

Culturedu mais en vue de la fabrication du sucre. 233

Tiges confites au sucre. . . . . . . . . 243

Éts non hurs. . . . . . . . . . . . . Ib.

Maïs frit. . . . . . . . . . . . Ib.

Maïs grillé. . . . . . . . . . . . . . 244

Mais farine froide. . . . . . . . . . . . 245

Maïs confit an vinaigre. . . . . . . . . . 246

Maïs confit au sucre. . . . . . . . . . 247

Mais blé doux. . . . . . . . . . . . Ib.

Maïs bouilli. . . . . ........ 48

Maïs-orgeat. . . . . . . . . . . . 249

Mais cru. . . . . . . . . . . . . 250

Grains de maïs. - Boisson. ....... Ib.

Chicha. . . . . . . . . . . Ib.

Petite bière. . . . . . . . . . 252

Posole. .. . . . . . . . . . . . . 253

Atextili. . . . . . . . . . . . 25 f

Tzenc. . . . . . . . . . 255

Café. . . . . . . . . . . . Ib.

Bière. . . . . . . . . . . . . 257

Eau de vie et vinaigre. . . . . . . . . . . 26 I 
Maís fn Grains entIflls. - Aliment. . . . 26il

Maîs rôti. . . . . . . . . . . . . 262

Mais en grains cassés. . . . . . . . . . . 2.63

Farine de la fuséc ou papeton. . . . . . I I .

Farine de mais. - Boisson........ 264

Clıocolatl. . . . . . . . . . . Ib.

Alimens. - Biscotes. . . . . . . . 265

Biscuit de mer. . . . . . . . . . . 266

Boulettes de maïs. . . . . . . . . . . 270

Chocolat oì la farine de mais remplace la fécule. 271

Crucliade. . . . . . . . . . . $27^{2}$

Galettes, gâteaux, pâtisseries. . . . . . . 276

Gaudes. . . . . . . . . . . . 281

Gaudes simples. . . . . . . . . . . . 282

Gaudes à la courge. . . . . . . . . . . 283

Gaudes aux pommes de terre. . . . . . . . Ib.

Gaufres. . . . . . . . . . . . 285

Gruau. . . . . . . . . . . . . I6.

Macaloni. . . . . . . . . . . . . 286

Millas ou millıs. . . . . . . . . . . 287

Maïs mondé.................. . Ib.

Miques. . . . . . . . . . . . . 288

Pain. . . . . . . . . . . . . . 291

Pain sans mélange. . . . . . . . . . 292

Pain mélangé. . . . . . . . . . . . . . $29^{6}$

Pain avec du froment. . . . . . . . . . 297

Pain avee moitié de seigle. . . . . . . . I6.

Pain avec du froment et du seigle. . . . . . . 2.95 
Pain avec de la farine de pomme de terre. . . $29^{8}$ Pain d'épice. . . . . . . . . . . . 302.

Pets de nomnes. . . . . . . . . . . . . . . . . . . . . . . . . . . . . . . . . .

Polenta. . . . . . . . . . . 304

Potages économiques. ........ . . 3 3 ı

Pouding. . . . . . . . . . . . 313

Poudres alimentaires. ........ 3 ז 6

Purée. . . . . . . . . . . . 3

Samp. . . . . . . . . . . . 320

Sauce. . . . . . . . . . Ib.

Semoule. . . . . . . . . Ib.

Tomton. . . . . . . . . . 321

Tôt-fait. . . . . . . . . . 322

Maïs-vernicelle. . . . . . . . . . . . . . 323

Note communiquée par mon confrère et ami,

M. le docteur Abel-Hibon. . . . . . 325

Emploi. . . . . . . . . . . . 328

Effets de la nourriture du maïs. . . . . . . 332

-- Chez l'homme. . . . . . . . . . . 337

- Chez les enfans en bas âge. . . . . . 343

- Chez les femmes qui allaitent. . . . . 345

Table des auteurs et des titres d'ourrages cités dans ce mémoire. . . . . . . . . . . . . . . 349

Explication des planches. . . . . . . . . . . . 359

Table nes matiéres. . . . . . . . . . 36 


\title{
CHEZ MADAME IUUARI, LIBRAIRE,
}

\author{
RUE DE L'ÉPEHON, No 7 .
}

Le Mals ou Blé de Turquie apprécié sons tous ses rapports; mémoire couronné le 25 août 1784 , par l'Académie des sciences, belles-lettres et arts de Bordeaux, par Pamentier. Paris, i8 12 . if f. 50 c. Supplénent au Menome de M. Panmenter sur le maïs; par M. le conte François de Neufchâteau. Paris, $1814-1817$, in-8. . . . . . $6 f$.

Di: lo'Agriculture ex Eunope ex ex Aménele, considérée et comparée dans les intérèts de la France ct de la monarchic; par P.-N.-H. Deby. Paris, 1825,2 vol. in-8.......... $10 \mathrm{fr}$.

L'A mi nes cultivate ns, ou Moyens simples et mis i la portée de tous les propriétaires, de tirer le meilleur parti des biens de campagne de toute espèce: par Poinsot. Paris, 1806,2 vol. in-8, fig. . 1 of.

Colrs de clltune; par A. Thoin, professem de culture au Musému d'histoire naturelle, avec un atlas in-4 de 65 planclies gravées représentant tous les outils, instrumens, ustensiles, machines et fabriques diverses, de grande et de petite culture, etc.; publié par Oscar Leclerc. Paris, 1827,3 vol. in-8, et atlas cart. . . . . . . . . . . 35 f.

Mancel pratique du lahocreur; par ChahouilléDupetitmont, cultivateur; $2^{e}$ édit. Paris, 1826 , 2 vol. in-12, fig. . . . . . . 8 8 .

Tuéatre d'agricleture et mesvage des champs d'Olivier de Serres, seigneur du Pradel, dans lequel est représenté tout ce qui est requis et nécessaire pour bien dresser, gouverner, enrichir et embellir la maison rustique: nonvelle édit. conforme an texte, augmentée de notes et d'un vocabulaire; publiće par la Société d'agriculture du département de la 
Seine. Paris, 1804 et 1805, 2 vol. in-4, fig., br. . . . . . . . . . $36 \mathrm{f}$.

Traití de la grande culture des terres, ouvrage utile aux personnes qui voudraient faire valoir de grandes exploitations; par Isoré. 1802,2 vol. in-12. . . . . . . . . . . . $3 \mathrm{f}$.

De l'Eau relativenent a l'écononie rustique, ou Traité de l'irrigation des prés; par J. Bertrand. 1 vol. in-12, fig. . . . . . . . . I f. 80 c.

Traité des prairies artificielles, ou Recherches sur les espèces de plantes qu'on peut cultiver avec le plus d'avantage en prairies artificielles, sur la culture qui leur convient le mieux; par H.-F. Gilbert; $6^{e}$ édition, augmentée de notes, par M. A. Yvart, et précédée d'une notice historique sur Gilbert, par M. Cuvier. Paris , 1826 , in-8. . . . . $5 \mathrm{f}$.

Traité géxéral de l'irrigation, contenant diverses méthodes d'arroser les prés et jardins, etc.; avec huit planches représentant diverses machines pour élever et conduire l'eau; par William Tatham ; traduit de l'anglais. Paris, 1805 , in-8. . . . 5 f.

'Traité général des prairies et de leur irrigation; par Ch. d'Ourches; deuxième édition. Paris, 1806 , in-8, fig. . . . . . . . . 4f. 50 c.

Plans raisonvés de toutes les espèces de Jardixs; par M. Gabriel Thoüin, cultivateur et architecte de jardins; troisième édit. Paris, ${ }_{1} 8_{2} 8$, in-fol. cart., figures, sable et eaux coloriés. . . . . $60 \mathrm{f}$. Entièrement coloriés. . . . . . . . . r oo f.

Ponologie puysiologroue, ou Traité du perfectionnement de la fructification, des movens d'améliorer les fruits domestiques et sauvages, de faire naittre des espèces et variétés nouvelles et d'en diriger la création, eic.; par M. Sageret. Paris, 183 o, in-8. 7f. 5 oc.

Tailie raisonvée des arbres fruitiers et autres opérations relatives à leur culture, démontrées clairement par des raisons physiques tirées de leur différente nature et de leur manière de végéter et de fructifier; par C. Butret; 18 édit. Paris, 1832 , in.12, fig. . . . . . . . . . . $\mathbf{f}$. 


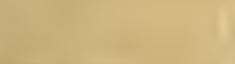

4
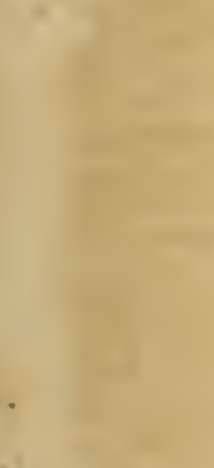

$+2$

$=$

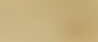

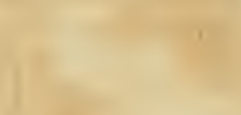

a

1

1

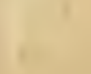




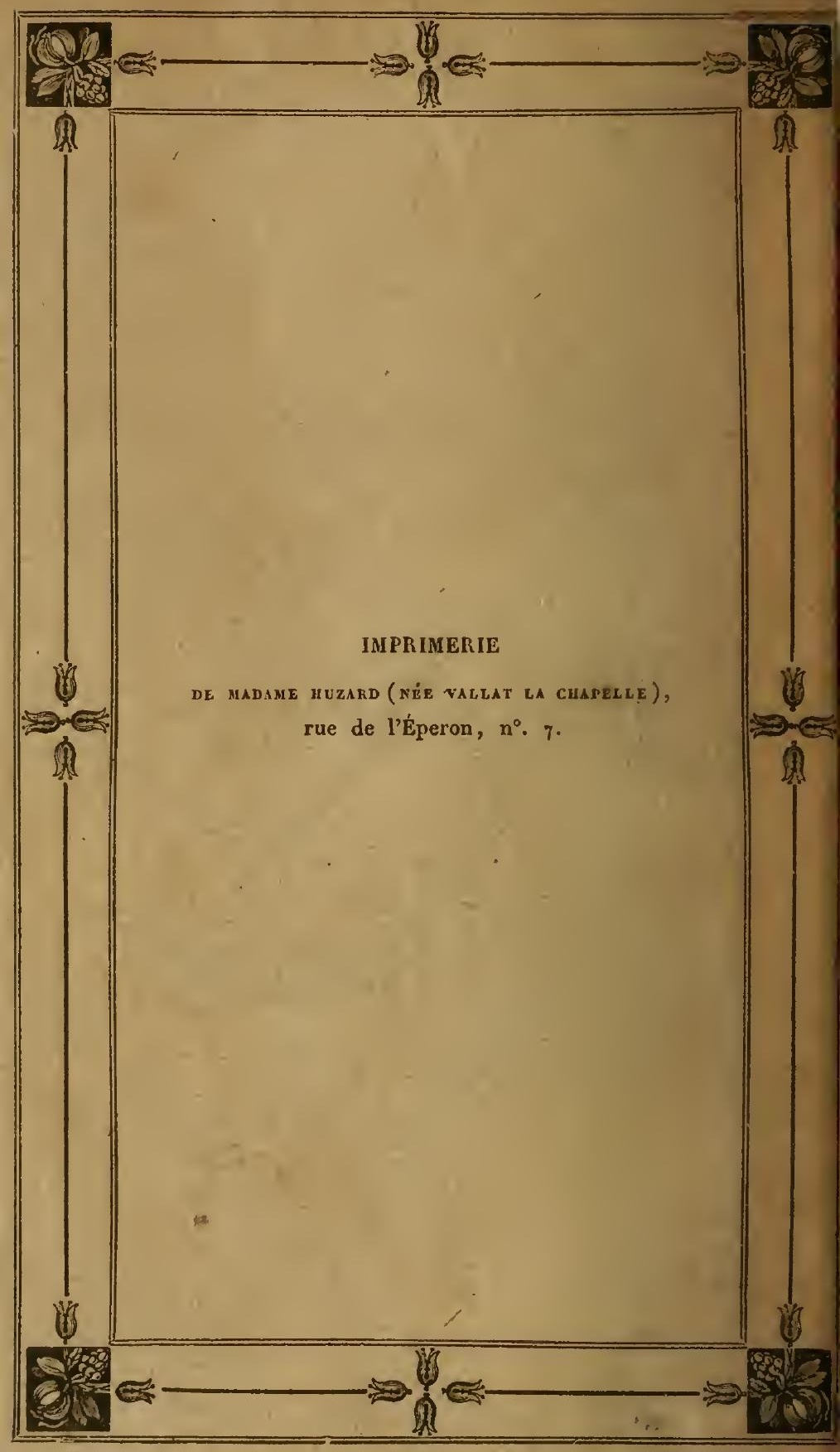




QL 34.241 D8
Duchesne, E.-A/Traite du mais ou $b$

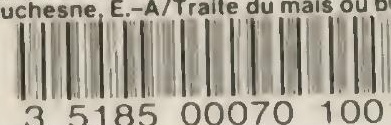

3518500070100 
